\title{
Nonlinear AtTitude and Formation Estimation of SpaCECRAFt AND Multi-Agent Systems
}

\author{
by \\ Michael Rososhansky \\ Master of Applied Science, Ryerson University (2010) \\ Bachelor of Engineering, Ryerson University (2007)
}

A dissertation

presented to Ryerson University

in partial fulfillment of the

requirements for the degree of

Doctor of Philosophy

in the program of

Aerospace Engineering

Toronto, Ontario, Canada, 2017

(C) Michael Rososhansky, 2017 


\section{AUTHOR'S DECLARATION FOR ELECTRONIC SUBMISSION OF A DISSERTATION}

I hereby declare that I am the sole author of this dissertation. This is a true copy of the dissertation, including any required final revisions, as accepted by my examiners.

I authorize Ryerson University to lend this dissertation to other institutions or individuals for the purpose of scholarly research.

I further authorize Ryerson University to reproduce this dissertation by photocopying or by other means, in total or in part, at the request of other institutions or individuals for the purpose of scholarly research.

I understand that my dissertation may be made electronically available to the public. 


\title{
Nonlinear AtTitude and Formation Estimation of SpaCECRAFt AND Multi-Agent Systems
}

\author{
Michael Rososhansky \\ Doctor of Philosophy, Aerospace Engineering, Ryerson University, Toronto (2017)
}

\begin{abstract}
This dissertation examines the state and parameter estimation problem of monolithic spacecraft and multi-agent systems in conjunction with the control algorithms. Nonlinear filtering techniques are investigated and applied to the problems of attitude estimation and control of monolithic spacecraft, distributed filtering for attitude estimation and control of satellite formation flying (SFF), and estimation and control of a multi-agent system in consensus tracking with uncertain dynamic model. The main objective is to investigate the performance of nonlinear filtering techniques under fault-free and fault-prone scenarios. In essence, the core of this research has been placed on identifying techniques to improve the efficiency and reduce the variance of estimations in nonlinear filtering. The research is primarily dedicated to the investigation of adaptive unscented Kalman filter (AUKF) and particle filter $(\mathrm{PF})$. A nonlinear filtering technique has been proposed for sequential joint estimation of a multi-agent system in consensus tracking with uncertain dynamic model. The new filter is called marginalized unscented particle filter (MUPF). The proposed filter uses the Rao-Blackwellised principle to couple the particle filtering technique with unscented transform algorithm.
\end{abstract}




\section{ACKNOWLEDGEMENTS}

This dissertation is a result of a continuous effort, over several years, in which I have experienced many uncertain and turbulent times. I owe a gratitude to many people who helped me along the way during the course of this journey. Without these people this dissertation would simply not be. First and foremost, I would like to express my heartfelt gratitude to Dr. Krishna Dev Kumar for his continuous support, guidance and motivation during this journey. Dr. Kumar, your guidance was indispensable to complete this dissertation. I would like to thank you for giving me the privilege to join and get some insight into your successful research group and for your skillful guidance in my own research. Furthermore, I would like to thank Dr. An-Min Zou for his guidance and discussions on estimation and control theory. I would also like to thank my colleagues Sobhan Etemadi, Afshin Rahimi, Alex Frias, Venkatesh Muthusamy and Min Adhikari. I enjoyed the fruitful discussions with you and spending time together. I also enjoyed discussions and collaboration with other researches worldwide Dr. Jesus Rooney Rivera Guillen and Waqas Manzur.

Next, I would like to thank my friends Oren Kats, Misagh Aghajani, Boris Miller

and Philip Milman. Thank you for your support, understanding and encouragement. I appreciate your friendship. Also, I would like to thank my dear family. My parents Boris and Mila, my brothers Ronen and Ariel, you are truly the most devoted people I ever knew. Thank you so much for listening and motivating me to finish this research. Finally, I would like to thank my wife Michelle for supporting me and helping me to finalize this journey. My love, I don't know how I can ever thank you for being such a caring and understanding person that you are. 
For ... my loving parents Boris and Mila Rososhansky

without whose support and endless love I would not have achieved this work. 


\section{TABle of Contents}

Author's Declaration ...................... ii

Abstract ....................... iii

Acknowledgements ................... iv

List of Tables . . . . . . . . . . . . . . . . . . $\mathrm{x}$

List of Figures . . . . . . . . . . . . . . . . xi

Nomenclature . . . . . . . . . . . . . . . . . xiv

Abbreviations .................... xvi

1 Introduction $\quad 1$

1.1 Research Motivation . . . . . . . . . . . . . . . . . . 2

1.2 Signal Noise . . . . . . . . . . . . . . . 4

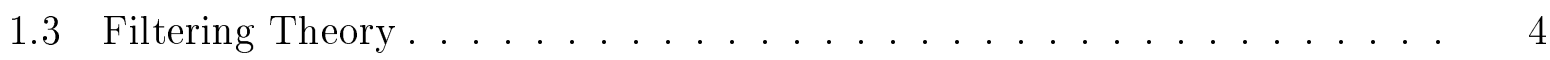

1.4 Linear and Nonlinear Filtering Techniques . . . . . . . . . . 6

1.4.1 Kalman Filter . . . . . . . . . . . . . . . . . . 6

1.4.2 Extended Kalman Filter . . . . . . . . . . . . . . . . 9

1.4.3 Unscented Kalman Filter . . . . . . . . . . . . . . . . . . . 10

1.4.4 Particle Filter . . . . . . . . . . . . . . . . . . 11

1.5 Problem Statement . . . . . . . . . . . . . . . . . . . . . 12

1.6 Research Objectives . . . . . . . . . . . . . . . . . . . . 13

1.7 Main Contributions . . . . . . . . . . . . . . . . . . . . 14

1.8 Thesis Outline . . . . . . . . . . . . . . . . . . 16

2 Nonlinear Filters and Neural Networks $\quad 17$

2.1 Introduction . . . . . . . . . . . . . . . . . . 17

2.2 Problem Statement . . . . . . . . . . . . . . . . . . . 18

2.3 Bayesian Estimation $\ldots \ldots \ldots \ldots \ldots$

2.4 Unscented Kalman Filter . . . . . . . . . . . . . . . . . . . . . . 22

2.5 Adaptive Unscented Kalman Filter . . . . . . . . . . . . . . . . . 24

2.6 Monte Carlo Approximation . . . . . . . . . . . . . . . . . 25 


\section{TABLE OF Contents}

2.7 Bayesian Importance Sampling $\ldots \ldots \ldots \ldots$

2.8 Particle Filter . . . . . . . . . . . . . . . . . . . . . . 28

2.9 Chebyshev Neural Networks . . . . . . . . . . . . . . . . . . 33

2.10 Concluding Remarks . . . . . . . . . . . . . . . . . 35

3 Attitude Estimation and Control of Monolithic Spacecraft 36

3.1 Introduction . . . . . . . . . . . . . . . . . . . . . . . . 37

3.1 .1 Literature review . . . . . . . . . . . . . . . . 38

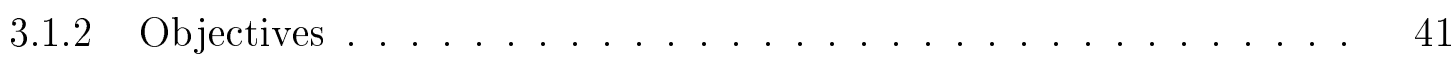

3.2 System Model, Filter Model, and Control Law . . . . . . . . . . . . . . 42

3.2 .1 Dynamic equation . . . . . . . . . . . . . . . 42

3.2 .2 Kinematic equations . . . . . . . . . . . . . . 42

3.2 .3 Filter models . . . . . . . . . . . . . . . . . 43

3.2 .4 Control law . . . . . . . . . . . . . . . . . 44

3.3 Simulation Results and Discussion . . . . . . . . . . . . . . . 45

3.3.1 Fault-free attitude estimation and control $\ldots \ldots \ldots \ldots 47$

3.3.2 Uncertainty in moment-of-inertia and external disturbances . . . . 56

3.3 .3 Control command faults . . . . . . . . . . . . . . . 64

3.3 .4 Sensor faults . . . . . . . . . . . . . . . . 67

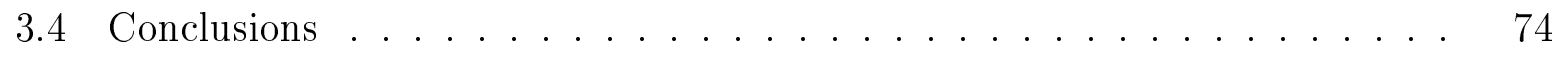

4 Distributed Attitude Estimation and Control of Spacecraft Formation $\begin{array}{ll}\text { Flying } & 75\end{array}$

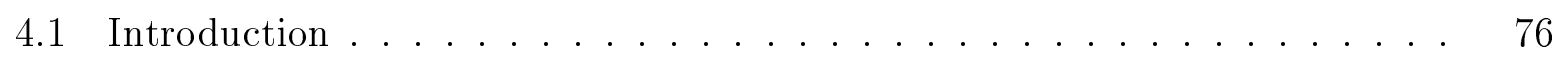

$4.1 .1 \quad$ Literature review . . . . . . . . . . . . . . . 78

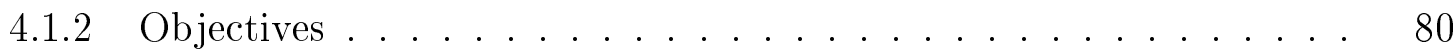

$4.2 \quad$ System Model, Filter Model, and Control Law . . . . . . . . . . . . 81

4.2 .1 Dynamic equations . . . . . . . . . . . . . . . . . 82

4.2 .2 Kinematic equations . . . . . . . . . . . . . . . 82

4.2 .3 Filter models . . . . . . . . . . . . . . . . . . 83

4.2 .4 Control law . . . . . . . . . . . . . . . . . 84 
4.3 Simulation Results and Discussion . . . . . . . . . . . . . . 84

4.3.1 Fault-free spacecraft formation flying . . . . . . . . . . 87

4.3.2 Uncertainty in moment-of-inertia and external disturbances . . . . . 89

4.3.3 Control command faults . . . . . . . . . . . . . . . 93

4.3.4 Measurements noise . . . . . . . . . . . . . 97

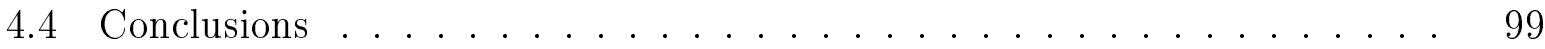

5 Online Estimation and Control of Multi-Agent Systems 100

5.1 Introduction . . . . . . . . . . . . . . . . . . 101

5.1 Literature review .................... 103

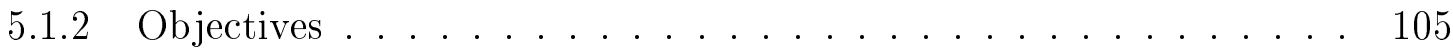

5.2 System Model, Filter Model, and Control Law . . . . . . . . . . . . 106

5.2.1 Dynamic equations ..................... 107

5.2 .2 Joint estimation model . . . . . . . . . . . . . 107

5.2 .3 Filter model . . . . . . . . . . . . . . . . . 108

5.2.4 Marginalized unscented particle filter . . . . . . . . . . . 109

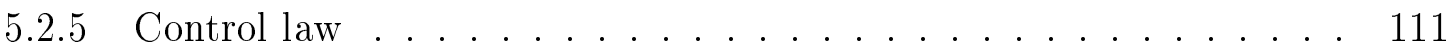

5.3 Simulation Results and Discussion . . . . . . . . . . . . . 113

5.3.1 Fault-Free multi-agent system . . . . . . . . . . . 115

5.3 .2 Uncertainty in dynamics model . . . . . . . . . . . 122

5.3.3 External disturbances . . . . . . . . . . . . . . 129

5.3 .4 Control command fault . . . . . . . . . . . . . 136

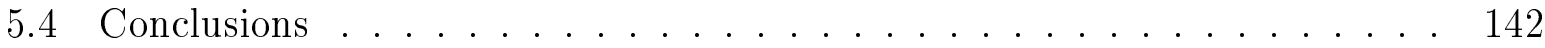

6 Conclusions $\quad 143$

6.1 Summary of Contributions . . . . . . . . . . . . . . . . . 144

6.1.1 Attitude estimation and control of monolithic spacecraft . . . . . 144

6.1.2 Distributed attitude estimation and control of spacecraft formation

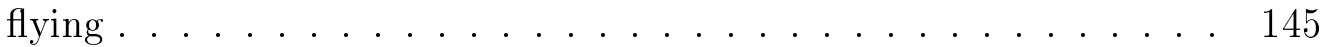

6.1.3 Online estimation and control of multi-agent systems . . . . . . . 146

6.2 Future Work . . . . . . . . . . . . . . 147 


\section{Table of Contents}

6.2.1 Proposal distributions ..................... 147

6.2.2 Resampling strategies . . . . . . . . . . . . 148

6.2.3 Improved nonlinear filtering techniques . . . . . . . . . . . . 148

6.3 Concluding Remarks . . . . . . . . . . . . . . . . 149

$\begin{array}{ll}\text { Appendices } & 150\end{array}$

$\begin{array}{ll}\text { A Importance Sampling } & 152\end{array}$

$\begin{array}{ll}\text { B Rao-Blackwellisation } & 153\end{array}$

C Parameter Estimation of Chebyshev Neural Network 155

C.1 Parameter estimation under fault-free scenario . . . . . . . . . . 156

C.2 Parameter estimation under uncertainty in dynamics model . . . . . . . 160

C.3 Parameter estimation under external disturbances . . . . . . . . . . . 164

C.4 Parameter estimation under constant applied control command . . . . . . 168

$\begin{array}{ll}\text { Bibliography } & 172\end{array}$ 


\section{List of Tables}

1.1 Summary of developments in filtering theory . . . . . . . . . . 7

3.1 Multinomial resampling algorithm . . . . . . . . . . . . . 46

3.2 Time required to enter error region . . . . . . . . . . . . . 55

3.3 Uncertainty in moment-of-inertia. . . . . . . . . . . . . 56

3.4 External disturbances. . . . . . . . . . . . . . . . . . 61

3.5 Constant applied torque. . . . . . . . . . . . . . 64

3.6 No control command during stabilization. . . . . . . . . . . 67

3.7 Random noise levels. . . . . . . . . . . . . . . 70

4.1 Initial attitude and angular velocity. . . . . . . . . . . . 85

4.2 Mass moment-of-inertia tensor for each spacecraft in the formation. . . . . 87

4.3 Filter mass moment-of-inertia tensor for each spacecraft in the formation. . $\quad 87$

4.4 Uncertainty in moment-of-inertia. . . . . . . . . . . . . . . 90

4.5 External disturbances. . . . . . . . . . . . . . . . . . 91

4.6 Constant applied control commands. . . . . . . . . . . . . 94

4.7 No control command during formation stabilization. . . . . . . . . . 96

4.8 Heterogeneous measurements. . . . . . . . . . . . . . . 98

5.1 Parameters of agent's dynamics . . . . . . . . . . . . . 115

5.2 Initial conditions. . . . . . . . . . . . . . . 116

5.3 Uncertainty in dynamic system. . . . . . . . . . . . . . 122

5.4 External disturbances. . . . . . . . . . . . . . . . 129

5.5 Constant applied control command. . . . . . . . . . . . . 136 


\section{List of Figures}

1.1 Fault Classification . . . . . . . . . . . . . . . . . . 3

2.1 System diagram . . . . . . . . . . . . . . . . . . . . . . . . . 19

2.2 Signal flow of unscented Kalman filter . . . . . . . . . . . . . . . 24

2.3 Particle filter representation of posterior distribution $\ldots \ldots$. . . . . . 29

3.1 Estimated angular velocities. . . . . . . . . . . . . . . . . . 47

3.2 Estimated attitude response using adaptive unscented Kalman filter. . . . . 48

3.3 Estimated attitude response using particle filter. . . . . . . . . . . . . 49

3.4 Spacecraft attitude error. . . . . . . . . . . . . . . . . . 51

3.5 Angular velocity residual. . . . . . . . . . . . . . . . 52

3.6 Root mean squared error for attitude response. . . . . . . . . . . . . 53

3.7 Root mean squared error for angular velocity. . . . . . . . . . . . . 54

3.8 Attitude response error under uncertainty in moment-of-inertia. . . . . . . 57

3.9 Angular velocity residual under uncertainty in moment-of-inertia. . . . . . 58

3.10 Root mean squared error of attitude response under uncertainty in moment-

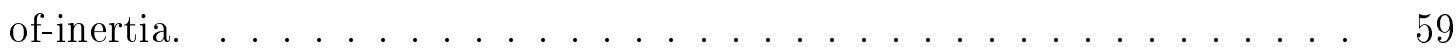

3.11 Root mean squared error of angular velocity under uncertainty in moment-

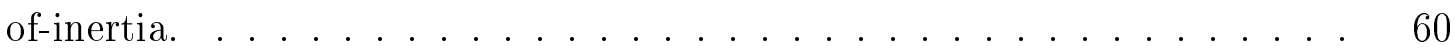

3.12 Attitude response error under external disturbances. . . . . . . . . . . 62

3.13 Angular velocity residual under external disturbances. . . . . . . . . . . . 63

3.14 Attitude response error under constant applied control commands. . . . . . 65

3.15 Angular velocity residual under constant applied control commands. . . . . 66

3.16 Attitude response error when no control command is applied during stabi-

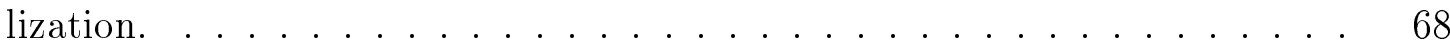

3.17 Angular velocity residual when no control command is applied during stabilization. . . . . . . . . . . . . . . . . . 69

3.18 Attitude response error under sensor fault. . . . . . . . . . . . . 72 
3.19 Angular velocity residual under sensor fault. . . . . . . . . . . . 73

4.1 Communication graph for spacecraft formation . . . . . . . . . . 81

4.2 Formation-keeping and station-keeping attitude errors. . . . . . . . . 88

4.3 Uncertainty in moment-of-inertia tensor. . . . . . . . . . . . . . 90

4.4 External disturbances. . . . . . . . . . . . . . . . . 92

4.5 Constant applied control commands. . . . . . . . . . . . 95

4.6 No control command during formation stabilization. . . . . . . . . . . 96

4.7 Heterogeneous measurements. . . . . . . . . . . . . . . 98

5.1 Communication graph for multi-agent system . . . . . . . . . . 106

5.2 Estimation of uncertain dynamic model under fault-free scenario . . . . . 117

5.3 State estimates . . . . . . . . . . . . . . . 118

5.4 Tracking error . . . . . . . . . . . . . . . . 119

5.5 Control input under fault-free scenario . . . . . . . . . . . . 120

5.6 Absolute error metric and relative error metric under fault-free scenario. . 121

5.7 Estimation of uncertain dynamic model . . . . . . . . . . . . . . . 124

5.8 State estimates under uncertainty in dynamics model . . . . . . . . 125

5.9 Tracking error under uncertainty in dynamics model . . . . . . . . . . 126

5.10 Control input under uncertainty in dynamics model . . . . . . . . . . . 127

5.11 Absolute error metric and relative error metric under uncertainty in dynamics model. . . . . . . . . . . . . . . . . 128

5.12 Estimation of uncertain dynamics model under external disturbances . . . 131

5.13 State estimates under external disturbances . . . . . . . . . . . 132

5.14 Tracking error under external disturbances . . . . . . . . . . . . 133

5.15 Control law under external disturbances . . . . . . . . . . . . . 134

5.16 Absolute error metric and relative error metric under external disturbances. 135

5.17 Estimation of uncertain dynamics model under a constant control command 137

5.18 State estimates under a constant control command . . . . . . . . . 138

5.19 Tracking error under a constant control command . . . . . . . . . . 139

5.20 Control input under a constant control command . . . . . . . . . . 140 
5.21 Absolute error metric and relative error metric under a constant control

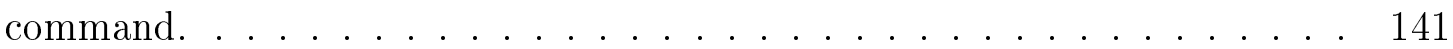

C.1.1Parameter estimation with adaptive unscented Kalman filter. . . . . . . . 157

C.1.2Parameter estimation with marginalized unscented particle filter. . . . . 159

C.2.1Parameter estimation under uncertainty of dynamic model using adaptive unscented Kalman filter. . . . . . . . . . . . . . . . . . . . 161

C.2.2Parameter estimation under uncertainty of dynamic model using marginalized unscented particle filter. . . . . . . . . . . . . 163

C.3.1Parameter estimation under external disturbances using adaptive unscented Kalman filter. . . . . . . . . . . . . . . . 165

C.3.2Parameter estimation under external disturbances using marginalized unscented particle filter. . . . . . . . . . . . . . 167

C.4.1Parameter estimation under constant applied control commands using adaptive unscented Kalman filter. . . . . . . . . . . . . . 169

C.4.2Parameter estimation under constant applied control commands using marginalized unscented particle filter. . . . . . . . . . . 171 


\section{NomenClATURE}

\section{List of lowercase symbols}

$\begin{array}{ll}f_{k} & \text { System transition function } \\ h_{k} & \text { measurement function } \\ k & \text { Time index } \\ n & \text { Number of states } \\ q_{d} & \text { Quaternion for desired attitude } \\ q_{e} & \text { Quaternion for attitude tracking error } \\ q_{i} & \text { Quaternion for attitude where } i=1,2,3,4 \\ \hat{q} & \text { Quaternion for estimated attitude } \\ \mathrm{x}_{k} & \text { State variable at time } k \\ \mathrm{x}_{k}^{(i)} & \text { Random sample } i \text { at time } k \\ \overline{\mathrm{x}} & \text { Mean value of } \mathrm{x} \\ \hat{\mathrm{x}} & \text { Estimate of } \mathrm{x} \\ \mathrm{y}_{k} & \text { Measurement at time } k \\ w_{k}^{(i)} & \text { Weight associated with particle } i \text { at time } k \\ \tilde{w}_{k} & \text { Normalized weight }\end{array}$

\section{List of uppercase symbols}

$C_{q_{e}} \quad$ Direction cosine matrix related to quaternion $q_{e}$

$J \quad$ Inertia matrix $\left(\mathrm{kg} \cdot \mathrm{m}^{2}\right)$

K Kalman Gain

$N_{p} \quad$ Number of random samples

$P_{x x} \quad$ Covariance of matrix $x$

$P_{x y} \quad$ Cross covariance matrix

$Q \quad$ Process noise matrix

$R \quad$ Measurement noise matrix 


\section{List of Greek symbols}

$\tau_{i} \quad$ Control torque for $i=1,2,3$

$\omega_{i} \quad$ Angular velocity for $i=1,2,3$

$\omega_{d} \quad$ Desired angular velocity

$\omega_{e} \quad$ Angular velocity error

$\hat{\omega}_{i} \quad$ Estimated angular velocity for $i=1,2,3$

$\hat{\chi}_{i} \quad$ Sigma points

\section{List of reference frames}

$\mathbb{B}-x, y, z \quad$ Spacecraft body-fixed coordinate frame

$\mathbb{E}-X_{I} Y_{I} Z_{I} \quad$ Earth centered inertial reference frame

(D) $-x_{0}, y_{0}, z_{0} \quad$ Local vertical local horizontal orbital reference frame

\section{Notational conventions}

$\forall i \quad$ For all values $i=1,2, \ldots, n$

$\mid \cdot \quad$ Absolute value

$\|\cdot\| \quad$ Euclidean norm for vectors, induced norm for matrices

$(\cdot) \quad$ Derivative of $(\cdot)$ with respect to time, $t$

$(\hat{\cdot}) \quad$ Estimation

$(\cdot) \quad$ Mean value

$(\tilde{\cdot}) \quad$ Estimation error

$\mathrm{x}^{\times} \quad$ Skew-symmetric matrix for vector $\mathrm{x}$

$\mathrm{x} \sim \mathrm{D} \quad \mathrm{x}$ is "distributed according" to $\mathrm{D}$.

$\mathrm{x} \approx \mathrm{y} \quad \mathrm{x}$ is "approximately equal" to $\mathrm{y}$.

$\mathrm{x} \propto \mathrm{y} \quad \mathrm{x}$ is "proportional" to $\mathrm{y}$.

$p(\mathrm{x} \mid \mathrm{y}) \quad$ The probability of event $\mathrm{x}$ given event $\mathrm{y}$ occurred. 


\section{AbBreviations}

\begin{tabular}{|c|c|}
\hline ACS & Attitude Control System \\
\hline $\mathrm{ARC}$ & Ames Research Center \\
\hline AUKF & Adaptive Unscented Kalman Filter \\
\hline $\mathrm{CNN}$ & Chebyshev neural network \\
\hline $\mathrm{CSA}$ & Canadian Space Agency \\
\hline ECI & Earth Centered Inertial frame \\
\hline $\mathrm{EKF}$ & Extended Kalman Filer \\
\hline FDI & Fault Detection and Isolation \\
\hline FUSE & Far Ultraviolet Spectroscopic Explorer \\
\hline i.i.d & Independent and Identically Distributed \\
\hline $\mathrm{KF}$ & Kalman Filter \\
\hline $\mathrm{MC}$ & Monte Carlo \\
\hline $\mathrm{MPF}$ & Marginalized particle filter \\
\hline MUPF & Marginalized unscented particle filter \\
\hline NASA & National Aeronautics and Space Administration \\
\hline $\mathrm{PDF}$ & Probability Distribution Function \\
\hline $\mathrm{PF}$ & Particle Filter \\
\hline $\mathrm{RBPF}$ & Rao-Blackwellized particle filter \\
\hline RW & Reaction Wheel \\
\hline RWA & Reaction Wheel Assembly \\
\hline SDE & Stochastic Differential Equations \\
\hline SIR & Sampling Importance Resampling \\
\hline SIS & Sequential Importance Sampling \\
\hline $\mathrm{SMC}$ & Sequential Monte Carlo \\
\hline $\mathrm{UKF}$ & Unscented Kalman Filter \\
\hline $\mathrm{UT}$ & Unscented Transform \\
\hline
\end{tabular}




\section{CHAPTER 1}

\section{Introduction}

pacecraft and multi-agent systems are primarily utilized to implement cross cutting addresses the problem of nonlinear attitude and formation estimation of monolithic spacecraft and multi-agent systems in sequential setting. Nonlinear filters provide a powerful means for estimating the hidden state of dynamic systems given the trajectory of measurements. Since most dynamic systems are inherently nonlinear, the application of nonlinear filtering spreads into several disciplines including communication, machine learning, system identification, finance, and many others. Various nonlinear filtering techniques are investigated with the primarily focus of estimating the posterior distributions of the state and parameters of space systems. The attention of this research is directed to (1) sequential attitude estimation of monolithic spacecraft with unknown angular velocity measurements and the presence of faults, (2) sequential estimation of unmeasurable angular velocity and reference angular acceleration of Spacecraft Formation Flying, and (3) sequential joint estimation of uncertain dynamic models which describe the evolution of a distributed multiagent system in consensus tracking.

Two nonlinear filtering techniques are investigated for online estimation of space systems, namely: adaptive unscented Kalman filter (AUKF) and sequential Monte Carlo (SMC). One of the attractive merits of AUKF is that it offers an easy approach for online estimation of Gaussian distributions instead of evaluating nonlinear dynamic models. In this sense, the AUKF uses a set of appropriately chosen weighted points to parameterize the mean and covariance of the posterior distributions. On the other hand, the SMC technique allow online estimation by combining the powerful Monte-Carlo sampling methods with Bayesian inference. The SMC approach has been widely applied to state and parameters estimation, for the former of which it is sometimes called particle filter (PF). In this disser- 
tation, SMC methods are referred to as particle filters. The basic idea of particle filter is to represent the posterior distributions by a number of independent random variables which are sampled directly from the state space and update the posterior once new measurements become available. The particles are properly located, weighted, and propagated recursively according to the Bayesian rule.

\subsection{Research Motivation}

In a world of limited resources, the development and launch of a successful spacecraft mission requires many scarce resources. Engineers, mathematician, scientists, computerprogrammers and professionals from many disciplines must be employed, countless tests must be performed, and changes must be continuously monitored. The cost of launching of a spacecraft start to accrue from the first stages of design, to manufacturing and assembly, and continue to increase during the life of the spacecraft's mission. As the owner of a spacecraft, corporations and government agencies would be interested in eliminating the risk of mission failure and reduce the probability of this scenario.

For example, the Far Ultraviolet Spectroscopic Explorer (FUSE) spacecraft was launched in 1999 with a total program cost of $\$ 120$ million. FUSE was developed in cooperation between NASA, the Canadian Space Agency (CSA), and the Centre National d'Etudes Spatiales of France [W. H. Moos 2000]. The FUSE was designed with four RW assemblies (RWA) used to control the attitude of the spacecraft and manage the angular momentum. Three wheels are arranged along the primary axes of the spacecraft, and a fourth, or skewed, wheel is oriented equidistant from the others. Although the prime scientific instrument of FUSE continue to perform well, the attitude control system (ACS) have suffered numerous failures, particularly with the Reaction Wheels (RWs) and gyroscopes which made science operation a challenge [Sahnow et al. 2006].

One year after launch, FUSE experienced a partial failure of ACS components, which started to affect the operation of the spacecraft. The first indication of a problem with a reaction wheel was received by the ground station in August 2000 when the pitch wheel showed signs of increased static friction. At the end of 2001, FUSE suffered the first 


\subsection{Research Motivation}

permanent component failure that had affected the ability to control the spacecraft. By 2006, the spacecraft was operating on a single reaction wheel as the three reaction wheels aligned with the principal axes of the spacecraft have also failed.

Various faults are possible in dynamic systems as presented in Figure 1.1. Abrupt and Permanent fault refer to changes in parameter value that occur much faster then the nominal process dynamics. Abrupt faults are relatively easy to detect since the fault effect is larger than the modeling uncertainty. Transient Fault results in a transient in system variables. Typically, transient behavior vanishes after a certain time have elapsed, and for certain faults no evidence of fault is observable in the measurements. Intermittent Fault refer to faults that occur at irregular intervals in a system that functions normally at other times. Intermittent faults are difficult to troubleshoot since each individual factor does not create the fault alone, so the factors can only be identified while system malfunction occurs. Incipient Fault are small and slowly developing faults. It is generally difficult to detect an incipient fault since the fault effect can be hidden in the uncertainty of the model. Finally, Additive Fault and Multiplicative Fault refer to faults that are either added or multiplied as an input to the system dynamics. The FUSE case stresses the need to develop online filtering techniques and control laws capable of controlling the dynamic systems under the presence of uncertain dynamic models and various fault scenarios.

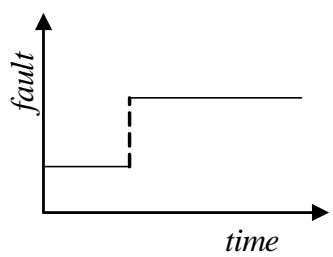

Abrupt and Permanent Fault

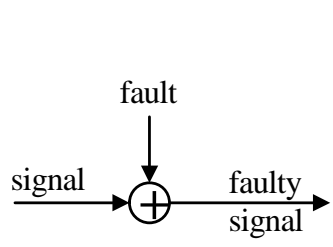

Additive Fault

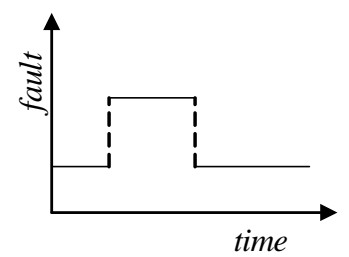

Transient Fault

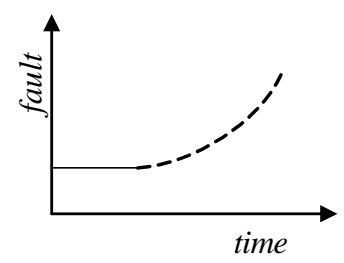

Incipient Fault

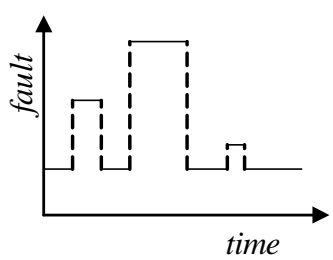

Intermittent Fault

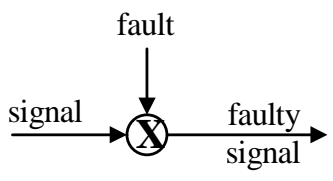

Multiplicative Fault

Figure 1.1: Fault Classification 


\section{$1.2 \quad$ Signal Noise}

Signals can be classified in categories depending on their features. In this thesis signals are divided onto deterministic signals and stochastic signal. The deterministic signal are not effected by random noise and there is no uncertainty about their values. Stochastic signals include an element of uncertainty which is usually removed through filtering process. The signal uncertainty is called signal noise which is a general term for unknown and generally unwanted distortions of the signal. The noise component in the system state is unpredictable and carry no useful information. In most practical engineering problems, the dynamic systems have traits that make them susceptible to noise. Therefore, the filtering process is needed to isolate the desired state from the unwanted noise in the signal. Generally, the signal noise is classified based on its statistical properties (sometimes called the "color" of the noise) and by how it modifies the intended signal. The color of the noise is distinguished by their spectral density i.e. white noise is a random signal having equal intensity at different frequencies, giving it a constant power spectral density. White noise has equal energy per cycle, meaning that its frequency spectrum is completely flat. Because white noise contains the frequencies of all other type of noise, it is frequently used to include all other noise type. White noise got its name from 'white light' since white light contains the frequencies of all color, combined together. If there was no noise in the state, then the state become deterministic and no filtering process is needed.

\section{$1.3 \quad$ Filtering Theory}

Estimation describes the process of finding an approximate to the state variable given the trajectory of observations. The estimation process is used to approximate the unobserved state on the basis of an observed state trajectory containing noise. The noise in the measurements means that there is a certain degree of uncertainty in the measurements. State Estimation refers to the study of estimating the states of a dynamic system from which we observe indirect noisy measurements. The dynamic system evolves as a function of time and incorporates a process noise which implies that the dynamic system cannot be modeled entirely in a deterministic settings. 


\subsection{Filtering Theory}

Filtering process is referred to the practice of estimating the hidden state of a dynamic system based on given observations, which are corrupted by random noise components ${ }^{1}$. The dynamic system follows a process known as the process model. Since, the hidden state cannot be measured directly, a related process called the measurement model is used to characterize the hidden state of the system.

The most outstanding and lasting developments in modern filtering theory can be found in numerous engineering applications where the hidden state is estimated from noisy measurements. The research in the field had started in late 1930's with the recasting and unifying of control and communication theories on statistical basis. This approach was possible due to the major contribution of Norbert Wiener ${ }^{2}$ and Andrey Kolmogorov's work during World War II [Wiener Norbert 1949]. In 1950's, researches had started relaxing the underlying assumptions of Wiener's contribution by replacing the covariance knowledge with state-space representation. This approach was possible due to the contribution of Rudolph E. Kalman [Kalman 1960], Ruslan Stratonovich, and Richard Battin ${ }^{3}$ [Battin 1961].

The next theoretical contribution to filtering theory was the introduction of stochastic differential equations (SDE) in the mid 1960's by Moshe Zakai who studied stochastic processes and their applications in information and control problems. Zakai [Zakai 1965] studied the filtering problem as a generalization of Kalman-Bucy filter and derived SDE for the posterior distribution which are modeled by a continuous Markov process. Zakai [Zakai 1969] derived a considerable simple solution for optimal filter, known as the Zakai equation, which has been the starting point for further research work in the field.

In 1990's researches had coupled the Monte Carlo technique with Bayesian algorithms. This technique is known as sequential Monte Carlo (SMC) and several closely related tech-

\footnotetext{
${ }^{1}$ The random noise component results from measurement inaccuracy, measurement distortions, model uncertainty etc. Without the noise component, the filtering problem would be deterministic and estimation would not be needed.

${ }^{2}$ Kolmogorov was a chief Russian exponent of the theory of probability while developing parallel probabilistic theories to Wiener's work. For many years Wiener and Kolmogorov were on each other's heels in terms of development of probabilistic theory. Both greatly contributed to the ideas of filtering problem although they were not aware of each others early work [Wiener Norbert 1949].

${ }^{3}$ Richard Battin published similar results to Rudolph E. Kalman in an internal MIT Instrumentation Laboratory Report in September 1961.
} 
niques have appeared in the scientific literature i.e., particle filter, Monte Carlo filter, condensation filter, bootstrap filter etc. The introduction of SMC has fundamentally influenced the area of nonlinear filtering; it has extended the reach of nonlinear filtering to higher dimensional application and enlarged the range of practical applications.

Table 1.1 present a brief historical development and contributions to filtering theory as arranged by year of development and contribution. It is unfortunately not been possible to include all leading researchers in the field and highlight all contributions. For extensive study and great historical overview of nonlinear filtering theory please refer to The Oxford Handbook of Nonlinear Filtering [Crisan \& Rozovskii 2011].

\subsection{Linear and Nonlinear Filtering Techniques}

The filtering techniques presented herein are organized according to the time of development, starting from Kalman filter (KF) and continuing with extended Kalman filter (EKF), unscented Kalman filter (UKF), and particle filter (PF). The description includes a short historical development, underling principle, and limitations of the filtering technique.

\subsubsection{Kalman Filter}

Historical Development: The celebrated Kalman Filter (KF) is a widely used filter for state estimation and prediction of linear systems. This well-known filter made the transition from a relatively abstract theory to application within a very short time during the early 1960's. The development of the filter resulted from continuous efforts by NASA during the 1950's to implement an estimator in space navigation problems. The primary objective was to develop the technology for a self-contained system which could be implemented in an on board digital computer. The system with pilot input was to guide the spacecraft from injection around the moon and to satisfy reentry corridor requirements on return to Earth. The research work on navigation was not proceeded very well at NASA and they needed a major breakthrough [Schmidt 1981]. During the late 1950's, work begun to replace the statistical based Wiener-Kolmogorov theory with state-space description. This resulted in work similar to Kalman filter as we know it today. Research 


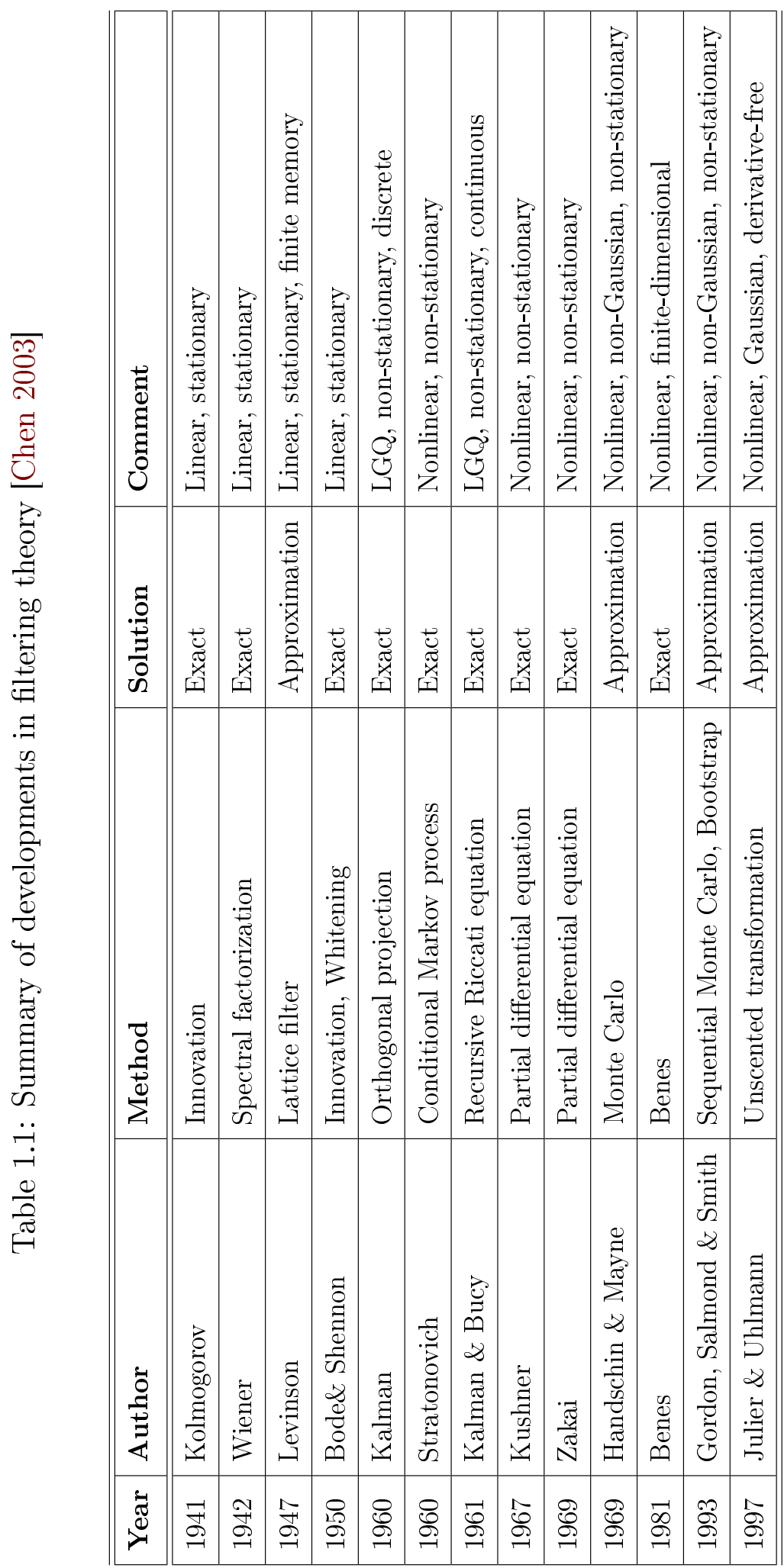


in this direction had started by Richard Bucy and James W. Follin [Bucy \& James 1962], A.G. Carlton [Carlton \& James 1956]. At the same time, Rudolph E. Kalman independently developed a discrete-time filter which he presented at NASA Ames Research Center (ARC). In 1960, Kalman and Bucy [Kalman \& Bucy 1961] became aware of each other's work and collaborated on the publication of a continuous-time Kalman filter. Additional historical notes on Kalman filter related to orbital dynamics and spacecraft guidance can be found in book by Richard Battin [Battin 1964]. The book by Dan Simon [Simon 2006] extensively elaborate on the discrete-time Kalman filter, continuous-time Kalman filter and optimal smoothing with Kalman filter. In 2009, Paul Zarchan and Howard Mussoff [Paul Zarchan 2000] published the third addition of progress in astronautics and aeronautics which extensively covers topic related to Kalman filtering and other filtering techniques.

Principle: The Kalman filter [Kalman 1960], also known as Kalman-Bucy filter, is an optimal linear filtering technique that represents a class of Bayesian estimator under conditions involving linear-Gaussian uncertainty. The KF is a model-based filter which yields state and parameter estimations based on the properties of the dynamic model. The development of the filter represents a corner stone in state estimation and relies on a two steps iterative process of prediction and correction. The prediction step involves a time update based on a one-step Markov process where the observation is predicted; and the correction step involve a measurement update where the correction to the estimate of current state is calculated.

Issues: The Kalman filter is limited to a range of linear dynamic models under the assumption of a Gaussian noise. Since most practical engineering systems possess some degree of nonlinearity, Kalman filter fail to provide an accurate state estimation. When applied to nonlinear systems large estimation errors are introduced in the process and the filter fails to converge [Martin E. Liggines 2009]. 


\subsection{Linear and Nonlinear Filtering Techniques}

\subsubsection{Extended Kalman Filter}

Historical Development: In 1960's, Rudolph E. Kalman was invited to National Aeronautics and Space Administration (NASA) Ames Research Center (ARC) in California. Kalman presented his results at ARC where his work believed to provide a practical solution to trajectory estimation and control. By early 1961, Kalman filter was implemented in computer simulations of the circumlunar navigation problem [Schmidt 1981]. The researchers at NASA realized that the filter could be divided into two parts: one part for time periods between sensor readings and another part for incorporating measurements. The effort at ARC accomplished several key steps in the development of Kalman filtering technique as a practical method for real-time onboard navigation of the Apollo mission. One of the major contributions was the development of the extended Kalman filter (EKF) to show that nonlinearities of the trajectory model did not compromise the accuracy of EKF for the ranges of errors expected during the Apollo missions [Grewal S. Mohinder 2010].

Principle: The EKF adapts the linear properties of Kalman filter to conditions involving nonlinearity by employing a local linearization using Taylor expansion ${ }^{4}$. The KF remains basic form of the EKF; however, partial derivatives are applied either to state transition or measurement models to develop a minimum variance estimate of the state.

Issues: The need to evaluate a Jacobian matrix to approximate the true density may cause the filter to diverge if the dynamic model is severely nonlinear. In addition, a Gaussian approximation can never describe the distribution well especially if the true density is non-Gaussian (i.e. binomial, heavily skewed etc.), [Arulampalam et al. 2002]. Finally, the EKF uses first-order terms for state approximation and ignores higher-order terms resulting in a large estimation errors as higher order terms begin to dominate.

\footnotetext{
${ }^{4}$ Taylor expansion is a representation of nonlinear functions as an infinite sum of terms that are calculated from the values of the function's derivatives with respect to a single point. For example, the best linear approximation for the function $f(\mathbf{x})$ is the first-order Taylor expansion $f(\mathbf{x}) \approx f(\mathbf{a})+f^{\prime}(\mathbf{a})(\mathbf{x}-\mathbf{a})$. Additional higher-order terms could be added to obtain a better approximation for $f(\mathbf{x})$ near the point $\mathbf{a}$ via the expression $f(\mathbf{x}) \approx f(\mathbf{a})+\frac{f^{\prime}(\mathbf{a})}{1 !}(\mathbf{x}-\mathbf{a})+\frac{f^{\prime \prime}(\mathbf{a})}{2 !}(\mathbf{x}-\mathbf{a})^{2}+\frac{f^{\prime \prime \prime}(\mathbf{a})}{3 !}(\mathbf{x}-\mathbf{a})^{3}+\cdots$.
} 


\subsubsection{Unscented Kalman Filter}

Historical Development: Unscented Kalman Filter (UKF) was first proposed by Simon Julier and Jeffrey K. Uhlmann [Julier \& Uhlmann 1997] and further developed by Wan and Van der Merwe [Wan \& Van Der Merwe 2000]. When the filter was developed at the Robotics Research Group (RRG) in Oxford, UK in 1994 it was called the "new filter". Due to the ambiguous and boring nature of the name, it was decided that a better name is needed for the filtering technique. Different names were identified for the filter and it was named Unscented Kalman Filter after a democratic vote by RRG members. In 1997, Julier and Uhlmann published the first paper describing the UKF as a new extension to Kalman filter for nonlinear systems.

Principle: The UKF provides an efficient derivative-free filtering technique for computing approximate solution of discrete-time nonlinear estimation problems. It is built on the principle that it is easier to approximate a probability distribution than it is to approximate an arbitrary nonlinear function [Julier \& Uhlmann 1997]. The UKF addresses the approximation issue through unscented transformation (UT) principle which is a deterministic sampling technique for selecting a set of specific sample points around the mean. These specific sample points are known as sigma points. When sigma points are propagated through nonlinear transition and measurement models, the mean and covariance of the state estimates can be accurately captured.

Issues: The UKF uses specific and deterministic sigma points to calculate the mean and covariance of the state of interest. These sigma points propagate through the nonlinear transition model and the posterior distribution is calculated by averaging the sigma points. The number of sigma points is small and may not adequately represent complicated posteriori distributions which may include biases such as skewness, kurtosis and outliers. The UKF is valid only when the posterior distribution can be closely approximated by a Gaussian distribution since the underlying principle of the UKF uses the Kalman filter as a backbone. The UKF preserves the linear update structure of the Kalman filter which is optimal only in linear Gaussian dynamic systems. 


\subsection{Linear and Nonlinear Filtering Techniques}

\subsubsection{Particle Filter}

Historical Development: The particle filter had its beginning in 1940's; however, only in 1993, Gordon, Salmond, and Smith [Gordon \& Smith 1993], presented the first application of a Monte Carlo resampling algorithm in Bayesian inference. The authors named the algorithm "The Bootstrap Filter" and demonstrated that bootstrap filter does not require any assumption about the state-space or system noise. In 1996, Kitagawa [Kitagawa 1996], [Kitagawa 1998] introduced similar results and named it the "Monte Carlo Filter". The term "particle filter" was first coined in 1996 by Pierre Del Moral [Del Moral 1996] in a published paper on nonlinear filtering. Bearing the nature of a recursive Bayesian estimation and sequential Monte Carlo simulation, particle filter has demonstrated its potential in various nonlinear, non-Gaussian, non-stationary estimation problems. The filter appears in the scientific literature under various names i.e., sequential importance sampling (SIS), bootstrap filtering, the condensation algorithm, interacting particle approximations, Monte Carlo filtering, sequential Monte Carlo (SMC) filtering, and survival of the fittest. As a result of its popularity several tutorials and books have been published on the subject [Arnaud Doucet 2001], [Arulampalam et al. 2002], [Chen 2003], [Doucet \& Johansen 2008].

Principle: The particle filter relaxes the Gaussian assumption of Kalman filters by approximating the posterior distribution with discrete random measures composed of weighted particles. The particles are samples of the hidden state and the weights are probability masses estimated using the Bayesian recursion. The filter implements sequential estimation by Monte Carlo simulation, where the samples drawn from the posterior distribution provide an approximation to the intractable integrals of the Bayesian equation.

Issues: The particle filter is guaranteed to diverge as time increases. To alleviate the divergence issue, a proper proposal distribution must be selected and additional resampling steps must be introduced in order to reduce the variance of estimation. The simplicity of the particle filtering technique is deceptive since the technique is extremely effective and stable; however, the Monte Carlo simulation increases the computation cost. 


\subsection{Problem Statement}

Nonlinear dynamic systems do not have an exact solution and various numerical approximation methods were proposed to address the intractability inherited in the Bayesian recursion. In contemplation of future space missions, it is of interest to estimate the state and parameters of space systems with strict demand for precise online estimation under the condition of nonlinearity, non-Gaussian and non-stationary models.

[PROB1] Nonlinear estimation. The inherent nonlinearity of the system models is identified as one of the technological bridges to be crossed for successful implementation of a nonlinear estimation and control algorithms. A linear representation of the dynamic model does not accurately describe the behavior of the space system for a wide range of operating conditions and results in system divergence over time.

[PROB2] Non-stationary estimation. Online estimation is a non-stationary process where the joint probability changes with respect to time. The non-stationary process will have a time-varying mean, time-varying variance, or both. The challenge is to precisely estimate the non-stationary dynamics of the space systems.

[PROB3] Uncertain dynamic models. One of the challenges is to estimate the posterior distributions of the space systems in online setting when the process and measurement models are unknown. This problem is known as system identification and is more concerned with parameter estimation rather than state estimation problem.

[PROB4] Estimation delay. A particular feature that affects the control of space systems is the availability of accurate estimations. One of the challenges for online estimation and control is to estimate the states in the presence of delayed measurements or absence of measurements.

[PROB5] Presence of faults. Space systems may experience various fault scenarios that cause the system to deviate from its nominal operational regime and degrade the mission success. These faults may occur at the dynamic models, control commands, or sensor measurements. 


\subsection{Research Objectives}

\subsection{Research Objectives}

The primarily objective is to investigate various nonlinear filtering techniques that provide precise state estimation for control of space systems in online setting. From practical perspective, the goal is to implement filtering techniques capable of estimating nonlinear, non-Gaussian and non-stationary processes. The following objectives are identified:

[OBJ1] Inverse problem: Nonlinear filtering techniques are investigated for applications in attitude estimation of monolithic spacecraft with unknown angular velocity measurements, estimation of unmeasurable angular velocity and reference angular acceleration of spacecraft formation flying, and sequential joint estimation of an uncertain dynamic models which describe the evolution of a distributed multi-agent system in consensus tracking. The filtering problem is treated as an inverse problem where the states of interest are estimated from available observations. The intention is to use the fundamentals of Bayesian algorithm to compute the posteriori distribution.

[OBJ2] Uncertain system dynamic modelling and estimation: The problem arises from an attempt to construct a mathematical model of an uncertain dynamic system given the measured data. System model is constructed sequentially through estimation of the system parameters in order to satisfy the requirements of online system tracking problem. The parameter estimation process determines the parameters of the system model with acceptable accuracy from sequential measurements, and the resulting model is by necessity an approximation of the true system dynamics.

[OBJ3] Validation through simulation and analysis: The performance of the proposed filters is examined in the presence of faults in the system dynamic model, control law and sensor measurements. We approach the validation of the proposed nonlinear filters from two perspectives: conventional theoretical analysis and numerical simulation study. Analytical studies are performed using adequate nonlinear filtering theory to demonstrate filter performance. Simulation studies are used as a design tool to demonstrate filter performance, compliance with design requirements, and validate established theoretical framework. 


\subsection{Main Contributions}

The contributions of this dissertation are aligned with the research objectives outlined in Section 1.6. The efforts are directed towards computation of posterior distributions of nonlinear, non-Gaussian, and non-stationary dynamic models in sequential setting. The posterior distribution are computed using nonlinear filtering techniques and are valid for state and parameter estimations and control. The results are addressed through numerical simulations under fault-free and fault-prone scenarios. The following research contributions are presented:

1. Online state and parameter estimation: Investigation of adaptive unscented Kalman filter and particle filter for state and parameter estimation serves the foundations of this research. The AUKF and its variants rely on parametric techniques to estimate the posterior distribution of the state and parameters ${ }^{5}$. In parametric techniques, a closed form solution is used to describe the posterior probability density function (i.e. Gaussian distribution or other well defined distribution). These parametric filters fail to converge if the dynamic system exhibits substantial nonlinearity and if the state and measurement noise are significantly non-Gaussian. These limitations have increased the research interest in alternative strategies to estimate any state and measurement equations. Since many practical system and measurement models are nonlinear and non-Gaussian, a significant challenge is to find an efficient estimation technique for online estimation and control from sequential observations. The alternative is to apply a category of non-parametric filtering ${ }^{6}$ techniques which approximate the posterior distribution by a finite number of random samples where each sample corresponds to a region in the state space.

\footnotetext{
${ }^{5}$ Parametric techniques assumes that the data have come from a type of probability distribution and makes inference about the parameters of the distribution. Type of probability distributions include: discrete distributions, continuous distributions, mixed discrete/continuous distributions, joint distributions etc.

${ }^{6}$ Non-parametric filters differ from parametric filters in that the model structure is not specified a priori but is instead determined from observations i.e. non-parametric filter makes no assumption about the probability distribution of the observations being assessed.
} 


\subsection{Main Contributions}

2. Attitude Estimation and Control of Monolithic Spacecraft: Accurate and stable online attitude estimation techniques are investigated in order to increase the pointing capability of a monolithic spacecraft. The attitude must be estimated from available noisy measurements in order to maintain a desired orientation of the spacecraft relative to a reference frame. The contribution of this study is the estimation of spacecraft attitude, in the absence of angular velocity measurements, using two nonlinear filtering techniques in an online setting. Here, the estimated state vector is comprised of four quaternion and three angular velocities.

\section{Distributed Attitude Estimation and Control for Spacecraft Formation} Flying: Nonlinear filtering techniques are investigated to obtain accurate estimations of angular acceleration and unmeasurable angular velocity for Spacecraft formation flying (SFF). The nonlinear filter coupled with an asymptotic output feedback attitude control law are utilized to estimate the unmeasurable angular velocity and angular acceleration of the SFF. Simulation results show fault reconstruction and recovery with formation stabilization in the presence of faults and uncertainty at the Spacecraft dynamic model, measurement model, and control commends. The performance comparison between the nonlinear filters is illustrated with numerical simulation through calculations of formation-keeping error and station-keeping error. Here, the estimated state vector is comprised of 36 states for a total of 6 spacecraft where for each spacecraft the attitude and angular velocities are estimated.

4. Online Estimation and Control of Multi-Agent Systems: A nonlinear filtering technique is developed for joint estimation which uses the Rao-Blackwellised principle to couple the particle filter with unscented transform (UT) technique. The proposed filter is applied in online joint estimation of an uncertain dynamic models, which describe the evolution of a distributed multi-agent system in consensus tracking. Chebyshev neural network (CNN) is incorporated as a universal approximation to learn an uncertain nonlinear functions of decentralized multi-agent dynamic system. This is a complex problem since the joint estimation vector is comprised of 180 states and parameters. 


\subsection{Thesis Outline}

The dissertation is structured as follows: Chapter 2 provides a formal description of nonlinear filtering problem and Bayesian framework which serves as a foundation for all nonlinear filters described in this dissertation. The chapter also provides a detailed overview and mathematical development of the nonlinear filters presented in this dissertation i.e. adaptive unscented Kalman filter (AUKF) and particle filter (PF). Chapter 3 deals with estimation of nonlinear attitude stabilization of monolithic spacecraft where the quaternion and angular velocity of a spacecraft are estimated under a finite-time control law. The chapter also provides an evaluation of performance and comparison between adaptive unscented Kalman filter and particle filter. The evaluation of performance and comparison focuses on calculations of quaternion error and angular velocity residuals and measured by the root mean squared error (RMSE). Chapter 4 covers the problem of online distributed filtering for velocity-free attitude estimation of spacecraft formation flying (SFF). The AUKF and $\mathrm{PF}$ in parallel with an asymptotic output feedback attitude control law are utilized to estimate the unmeasurable angular velocity and angular acceleration of Spacecraft in formation flying. Here, the performance comparison between AUKF and PF is illustrated with numerical simulation of formation-keeping error and station-keeping errors. Chapter 5 deals with online estimation of uncertain dynamic function that describes the evolution of a decentralized multi-agent system in consensus tracking under bounded external disturbances, and process and measurement noise. Here, we develop a nonlinear filter for online estimation which uses the Rao-Blackwellised technique to couple the particle filtering algorithm with unscented transform (UT) principle. The filter is applied for system identification using a Bayesian neural network (NN) learning where the parameters of the NN are estimated using the AUKF and the proposed filter. Finally, the dissertation is concluded in Chapter 6 with summary of accomplishments and suggestions for future work. I hope this dissertation will provide a greater exposure to the reader on the field of nonlinear estimation and will constitute a valuable source of information of various nonlinear filtering techniques which are applicable in the aerospace industry. 


\section{CHAPTER 2}

\section{Nonlinear Filters and Neural Networks}

"The greatest physical tradition of Newton had necessarily been one of determinism, where a perfect knowledge of the universe at one instant is understood to involve a perfect knowledge of its history throughout all time. It would have been Newton's assumption that to give the present positions and speeds of the particles in a wave moving across a surface of the Charles [Charles River in Massachusetts] would allow us to plot the movement of this wave forever. Unfortunately, no perfect knowledge of the present is available to us with our limited measuring instruments, and the problem that faces the working physics is to find how far one can go with the imperfect knowledge. For this it is required to work not with a single, fixed universe but with many different universes simultaneously, each having some preassigned probability. It is not possible to tell what will happen in the future but what may happen at certain times, given certain conditions" [Wiener Norbert 1956].

\subsection{Introduction}

Nonlinear filtering theory deal with the problem of estimating the hidden state of a time varying dynamic system given access to measurements which are corrupted by a random noise component. The time varying dynamic system evolve according to a stochastic process known as a process model, or system model, which can not be measured directly. Instead, a measurement model, is used to correlate the desired hidden state to the measurements. The filtering process is an Inverse problem since the state of interest are estimated from available measurements, where probability analysis are used to estimate the posterior distributions of the state. The posterior distributions provide a solution to filtering problem based on Bayesian algorithm. 
The filtering problem was first established in the early 1940s given the pioneering work of Norbert Wiener and Andrey N. Kolmogorov [Wiener Norbert 1949]. Wiener was first to estimate dynamic systems in the presence of noise in continuous-time domain whereas Kolmogorov studied the problem in discrete-time domain. The result of Wiener were included in a classified Defence Research Council report issued in January 1942. The report is known as "The Yellow Peril" because of the yellow cover of the book.

This chapter explains the filtering problem and provides a mathematical treatment of nonlinear filtering techniques and Chebyshev neural networks (CNN). In Section 2.2, the filtering problem is formally defined in discrete-time domain. Section 2.3 covers Bayesian estimation theory which is the foundation to all nonlinear filtering techniques discussed in this dissertation. Section 2.4 introduces the well documented unscented Kalman filter (UKF) algorithm. Section 2.6 and Section 2.7 are crucial foundations for proper design of Sequential Importance Sampling (SIS) algorithm which is described in Section 2.8. Finally, Section 2.9 provides a mathematical description of Chebyshev neural networks.

\section{$2.2 \quad$ Problem Statement}

The problem of nonlinear filtering consists of computing the conditional distribution of the state variables at current time given the measured data accumulated up to that time. The hidden state variable $\mathbf{x}_{k}$ is not directly measured and need to be reconstructed from the measured data $\mathbf{y}_{k}$. The state-space representation provides a convenient and compact way to model and analyze the estimation problem with multiple inputs and outputs. To define the estimation problem, system model is required to describe the evolution of the dynamic system with respect to time. In addition, measurement model is needed to relate the noisy measurements to the state. The system model is a one-step Markov process that describes the evolution of the state from $\mathbf{x}_{k}$ to $\mathbf{x}_{k+1}$; and, the measurement model relates the state vector to the measurement vector, $\mathbf{y}_{k}$. Figure 2.1 shows the system diagram.

For online estimation and control problems, the interest is in a discrete-time models given its suitability for digital computation. A general discrete-time estimation problem in state- 
space representation is given as follows [Jazwinski 1970]:

$$
\begin{aligned}
\mathbf{x}_{k+1} & =f_{k}\left(\mathrm{x}_{k}, u_{k}\right)+w_{k} \\
\mathbf{y}_{k} & =h_{k}\left(\mathrm{x}_{k}\right)+v_{k}
\end{aligned}
$$

where Equation (2.1a) represents the system model, and Equation (2.1b) is the measurement model. The function $f_{k}: \mathbb{R}^{n_{x}} \times \mathbb{R}^{n_{v}} \rightarrow \mathbb{R}^{n_{x}}$, describes the state evolution and can be either a linear or nonlinear ${ }^{1}$. The function $h_{k}: \mathbb{R}^{n_{x}} \times \mathbb{R}^{w_{n}} \rightarrow \mathbb{R}^{n_{y}}$ describes the measurement model. The subscript $k$ in $f_{k}$ and $h_{k}$ indicates that the models themselves may be time varying. $\mathbf{x}_{k} \in \mathbb{R}^{n_{x}}$ is the state vector which gives a complete information of the system behaviour, and is necessary for control purpose, $\mathbf{y}_{k} \in \mathbb{R}^{n_{y}}$ is the measurement vector, $u_{k}$ is a deterministic control input, $w_{k}$ is an additive process noise, and $v_{k}$ is an additive measurement noise.

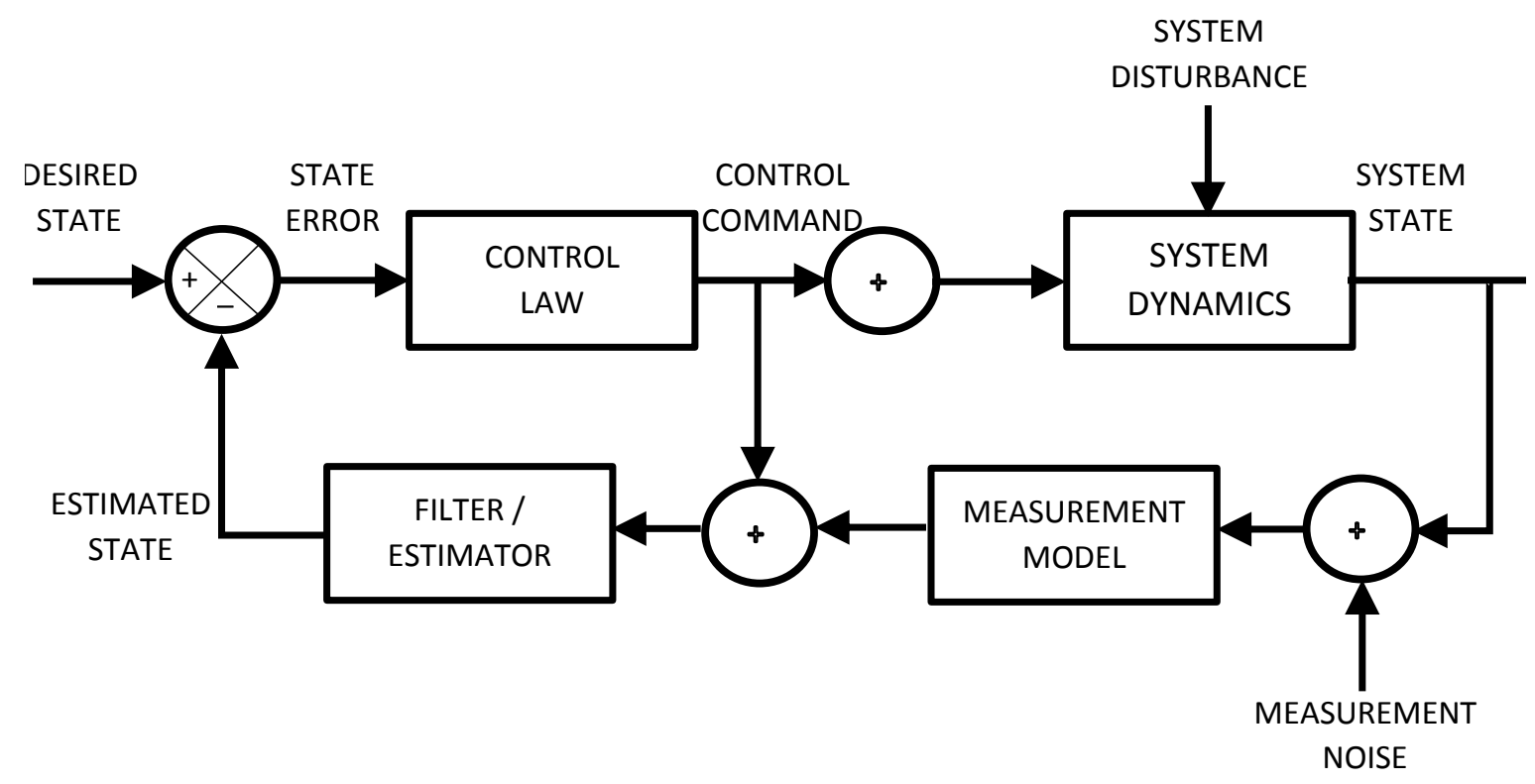

Figure 2.1: System diagram

\footnotetext{
${ }^{1}$ In case of linear function, the estimation problem is simplified significantly and Kalman filter can be implemented (see Chapter 1.4.1). For nonlinear functions, where the relationship between system model and measurement model breaks down, nonlinear filters have been developed i.e., unscented Kalman filter (UKF) or particle filter (PF).
} 


\subsection{Bayesian Estimation}

The state estimation involves the construction of a posterior probability density function (pdf) which describes the state of an evolving dynamic system. The posterior pdf, denoted as $p\left(\mathbf{x}_{k} \mid \mathbf{y}_{k}\right)$, is the conditional probability of the state that is constructed from the prior distribution and the likelihood function according to Bayesian rule. Knowledge of the posterior distribution enable to extract the hidden state from the uncertain measurements. Defining the posterior distribution and extract the hidden state, $\mathbf{x}_{k}$, from noisy measurement $\mathbf{y}_{k}$ using Bayesian rule requires three characteristics: [Martin E. Liggines 2009]

1. Prior distribution, denoted as $p\left(\mathbf{x}_{k} \mid \mathbf{y}_{k-1}\right)$, includes the historical trajectory of the system state. The prior distribution is called a "prior" since the state distribution is known before an evidence of measurement is taken into account.

2. Likelihood function, denoted as $p\left(\mathbf{y}_{k} \mid \mathbf{x}_{k}\right)$, characterizes the information of sensor measurements and available observations of the dynamic system.

3. Evidence, denoted as $p\left(\mathbf{y}_{k} \mid \mathbf{y}_{k-1}\right)$, characterizes the evidence obtained from the latest measurement.

The detailed derivations of the recursive Bayesian estimation is developed by making the following assumptions:

1. The state vector, $\mathbf{x}_{k} \in \mathbb{R}^{n}$, evolves according to the system model in Equation (2.1a); where the probabilistic nonlinear filtering model consists of a sequence of conditional probability distribution such that $\mathbf{x}_{k} \sim p\left(\mathbf{x}_{k} \mid \mathbf{x}_{k-1}\right)$. Here, $p\left(\mathbf{x}_{k} \mid \mathbf{x}_{k-1}\right)$ is the probabilistic model of the dynamic system.

2. The measurements are related to the state vector via the measurement model in Equation (2.1b); where the probabilistic nonlinear model consists of a sequence of conditional probability distribution such that $\mathbf{y}_{k} \sim p\left(\mathbf{y}_{k} \mid \mathbf{x}_{k}\right)$. Here, $p\left(\mathbf{y}_{k} \mid \mathbf{x}_{k}\right)$ is the probabilistic model of the measurements given the state.

3. The state of the dynamic system follows a first-order Markov process, $p\left(\mathbf{x}_{k} \mid \mathbf{x}_{0: k-1}\right)=$ $p\left(\mathbf{x}_{k} \mid \mathbf{x}_{k-1}\right)$; where the Markov property implies that the conditional probability dis- 


\subsection{Bayesian Estimation}

tribution of $\mathbf{x}_{k}$ depends only upon the given distribution $\mathbf{x}_{k-1}$ and not on the sequence of states that preceded it.

4. The measurements $\left\{\mathbf{y}_{k} ; k \in \mathbb{N}\right\}$ are conditionally independent of the given state $\left\{\mathbf{x}_{k} ; k \in \mathbb{N}\right\}$ and of the marginal distribution $p\left(\mathbf{y}_{k} \mid \mathbf{x}_{k}\right)$.

5. The initial pdf $p\left(\mathbf{x}_{1} \mid \mathbf{y}_{0}\right)=p\left(\mathbf{x}_{1}\right)$ of the state vector is available together with the system model $f_{k}$ and measurement model $h_{k}$.

The posterior pdf is obtained recursively in two stages: prediction and update. Suppose the pdf $p\left(\mathbf{x}_{k-1} \mid \mathbf{y}_{k-1}\right)$ at time step $k-1$ is available. The prediction stage incorporate the system model (Eq'n (2.1a)) to obtain a distribution of the state at time step $k$ via the Chapman-Kolmogorov Equation [Chen 2003].

$$
p\left(\mathbf{x}_{k} \mid \mathbf{y}_{k-1}\right)=\int p\left(\mathbf{x}_{k} \mid \mathbf{x}_{k-1}\right) p\left(\mathbf{x}_{k-1} \mid \mathbf{y}_{k-1}\right) d \mathbf{x}_{k-1}
$$

Then at time step $k$ a measurement $\mathbf{y}_{k}$ becomes available and may be used to update the prior via Bayes rule

$$
p\left(\mathbf{x}_{k} \mid \mathbf{y}_{k}\right)=\frac{p\left(\mathbf{y}_{k} \mid \mathbf{x}_{k}\right) p\left(\mathbf{x}_{k} \mid \mathbf{y}_{k-1}\right)}{p\left(\mathbf{y}_{k} \mid \mathbf{y}_{k-1}\right)}
$$

In order to compute the posterior distribution, $p\left(\mathbf{x}_{k} \mid \mathbf{y}_{k}\right)$, in the update stage, the denominator involve the term $p\left(\mathbf{y}_{k} \mid \mathbf{y}_{k-1}\right)$ known as the evidence equation must be solved. The evidence equation is determined by the following integral

$$
p\left(\mathbf{y}_{k} \mid \mathbf{y}_{k-1}\right)=\int p\left(\mathbf{y}_{k} \mid \mathbf{x}_{k}\right) p\left(\mathbf{x}_{k} \mid \mathbf{y}_{k-1}\right) d \mathbf{x}_{k}
$$

Conceptually, the Bayesian approach for recursive estimation is simple to solve; however, the challenge is to evaluate the integrals in the Bayesian equation. The evaluation of the integrals is very difficult and can not be determined analytically since the large dimensionality of the integral causes numerical integration techniques to break down. Several analytical solutions were developed to deal with the Bayesian equation under unique conditions. In particular, if the evolution process (Equation (2.1a)) and measurement process (Equation (2.1b)) are linear, and $\mathbf{x}_{0}, v_{k}$ and $w_{k}$ are additive, independent and Gaussian then the Kalman Filter (KF) provide an optimal solution; otherwise, other approaches have to be implemented. This chapter covers few of the proposed approaches starting with a 
basic overview of Unscented Kalman Filter in Section 2.4. A detailed work on Bayesian filtering is presented in the following books by Doucet \& Gordon [Arnaud Doucet 2001], James Candy [Candy 2009], and Simo Särkkä [Särkkä 2013].

\subsection{Unscented Kalman Filter}

The Unscented Kalman Filter (UKF) has been widely used in many engineering applications incorporating both linear and nonlinear system dynamics. General application area of UKF may be divided into state estimation, parameter estimation and joint estimation. The filter has been proposed by Simon Julier and Jeffrey K. Uhlmann [Julier \& Uhlmann 1997]. The working mechanics of UKF involves propagation of a Gaussian random state vector $\mathbf{x}_{k}$ through the nonlinear measurement model $h_{k}$, to obtain a transformed random observation vector $\mathbf{y}_{k}$ [Wan-Chun Li 2005]. The goal is to accurately estimate the mean value of the observation $\overline{\mathbf{y}}_{k}$ and the covariance $P_{\mathbf{y y}}$ of the observation $\mathbf{y}_{k}$ at time $k$. The UKF uses the unscented transform process to generate a vector of sigma points whose mean and covariance is a close approximation of the actual system state. The unscented transform process involves three steps: First, for a given state vector $\mathbf{x}_{k}$ with $N_{x}$ random variables, a set of $\left(2 N_{x}+1\right)$ sigma points are generated around $\hat{\mathbf{x}}_{k}$ along with a set of associated weights. The sigma points are propagated through the nonlinear measurement model $h_{k}$ to obtain the vector of observations $\mathbf{y}_{k}$. Next, the mean vector $\overline{\mathbf{y}}_{k}$ and covariance matrix $P_{\mathbf{y y}}$ of the observation vector $\mathbf{y}_{k}$ are calculated based on the distribution of these deterministic sigma points and their weights. The UKF formulation is presented below [Rogers 2007].

\section{UNSCENTED KALMAN FILTER (UKF)}

\section{Compute weights}

$$
\begin{aligned}
\mathbf{W}_{0}^{(m)} & =\frac{\lambda}{n+\lambda}, \\
\mathbf{W}_{0}^{(c)} & =\frac{\lambda}{n+\lambda}+\left(1-\alpha_{U K F}^{2}+\beta_{U K F}\right), \\
\mathbf{W}_{i}^{(m)} & =\frac{1}{2(n+\lambda)}, \quad i=1, \ldots, 2 n \\
\mathbf{W}_{i}^{(c)} & =\frac{1}{2(n+\lambda)}, \quad i=1, \ldots, 2 n
\end{aligned}
$$


2. Establish symmetric sigma points about the state estimate

$$
\mathcal{X}_{k-1}^{(i)}= \begin{cases}\hat{\mathbf{x}}_{k-1} & i=0 \\ \hat{\mathbf{x}}_{k-1}+\sqrt{(n+\lambda) P_{i}} & \forall i=1,2, \ldots, n \\ \hat{\mathbf{x}}_{k-1}-\sqrt{(n+\lambda) P_{i}} & \forall i=n+1, n+2, \ldots, 2 n\end{cases}
$$

3. Instantiate sigma points through the process model

$$
\hat{\mathcal{X}}_{k}^{(i)}=f_{k}\left(\mathcal{X}_{k-1}^{(i)}\right)
$$

4. Predict mean and covariance of states

$$
\begin{aligned}
\overline{\mathbf{x}}_{k} & =\sum_{i=0}^{2 n} W_{i}^{(m)} \hat{\mathcal{X}}_{k}^{(i)} \\
P_{x x} & =\sum_{i=0}^{2 n} W_{i}^{(c)}\left[\hat{\mathcal{X}}_{k}^{(i)}-\overline{\mathbf{x}}_{k}\right]\left[\hat{\mathcal{X}}_{k}^{(i)}-\overline{\mathbf{x}}_{k}\right]^{T}+Q
\end{aligned}
$$

5. Instantiate sigma points through the measurement model

$$
\hat{\mathcal{Y}}_{k}^{(i)}=h_{k}\left(\mathcal{X}_{k-1}^{(i)}\right)
$$

6. Predict mean and covariance of measurements

$$
\begin{aligned}
\overline{\mathbf{y}}_{k} & =\sum_{i=0}^{2 n} W_{i}^{(m)} \hat{\mathcal{Y}}_{k}^{(i)} \\
P_{y y} & =\sum_{i=0}^{2 n} W_{i}^{(c)}\left[\hat{\mathcal{Y}}_{k}^{(i)}-\overline{\mathbf{y}}_{k}\right]\left[\hat{\mathcal{Y}}_{k}^{(i)}-\overline{\mathbf{y}}_{k}\right]^{T}+R
\end{aligned}
$$

7. Predict cross covariance

$$
P_{x y}=\sum_{i=0}^{2 n} W_{i}^{(c)}\left[\hat{\mathcal{X}}_{k}^{(i)}-\overline{\mathbf{x}}_{k}\right]\left[\hat{\mathcal{Y}}_{k}^{(i)}-\overline{\mathbf{y}}_{k}\right]^{T}
$$

8. Gain calculation and update

$$
\begin{aligned}
K & =P_{x y} P_{y y}^{-1} \\
\hat{\mathbf{x}}_{k} & =\overline{\mathbf{x}}_{k}+K\left(\mathbf{y}_{k}-\overline{\mathbf{y}}_{k}\right) \\
P^{+} & =P_{x x}-K P_{y y} K^{T}
\end{aligned}
$$


The UKF parameter $\lambda$ in Equation (2.5) is a composite scaling parameter defined by $\lambda=\left(\alpha_{U K F}\right)^{2}(n+\kappa)-n$, where $\alpha_{U K F}$ and $\kappa$ determine the spread of the sigma points around the mean, $n$ is the dimension of the augmented state and $\beta_{U K F}$ is a design parameter for the distribution [Wan \& Van Der Merwe 2000]. The term $\sqrt{(n+\lambda) P_{i}}$ in Equation (2.6) represent the scaled $i^{t h}$ row/columns of the sqre root factor of $P^{-}$. Figure 2.2 shows the signal flow diagram of the unscented Kalman filter. It is evident that the sigma points are propagated through the process and measurements models after which the state and measurements statistics are estimated.

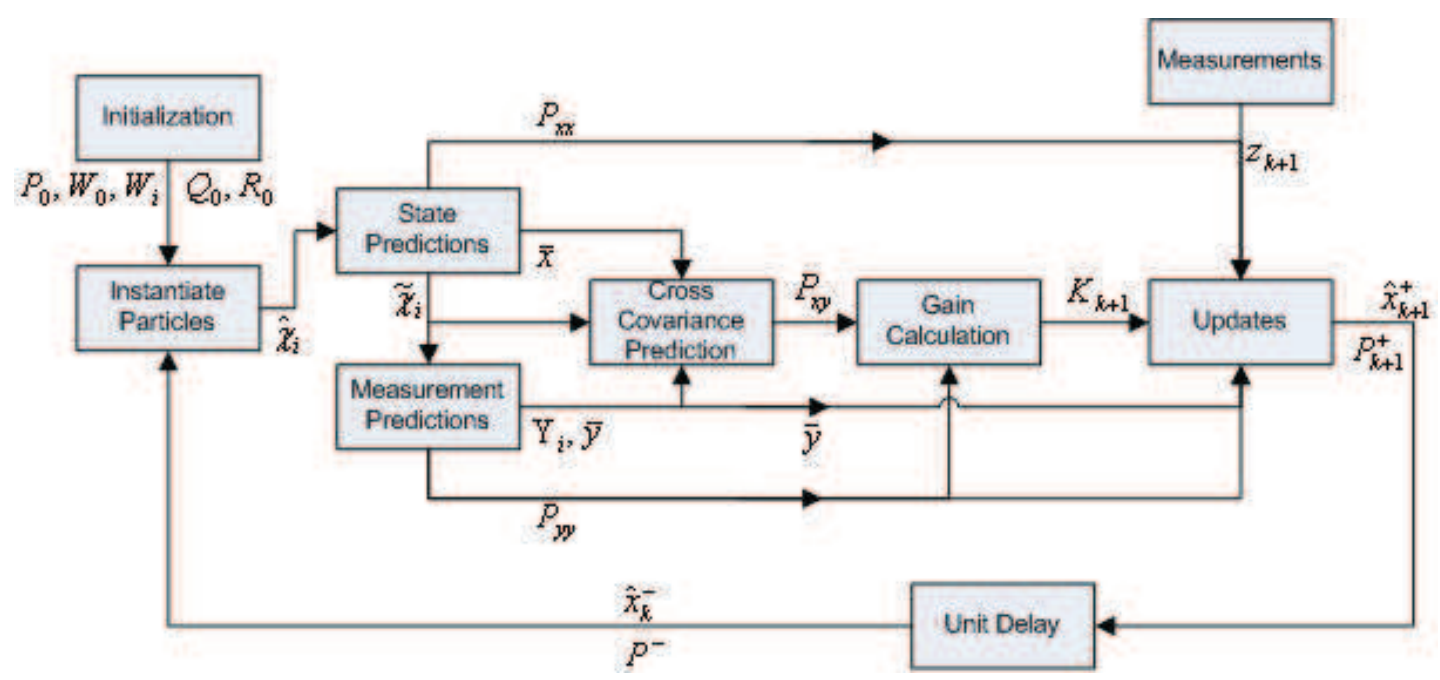

Figure 2.2: Signal flow of unscented Kalman filter [Abreu 2011]

\subsection{Adaptive Unscented Kalman Filter}

The following adaptive mechanism is used to adjust the process noise covariance matrices [Abreu 2011].

$$
P_{x x}=P_{k}^{-} \varepsilon+Q_{k+1}
$$

If $\varepsilon=1$ then the standard KF prediction occurs, if $\varepsilon>1$ the filter will weight the data exponentially so that the effect of current data is emphasized and information from older measurements is discounted, hence the name 'fading factor' or 'fading memory'. 


$$
\varepsilon=1+\frac{\operatorname{trace}\left(R_{k}\right)}{\zeta}
$$

This formulation guarantees that the adaptive factor will increase for larger values of $R_{k}$. The trace operation consists of the sum of the diagonal elements of a matrix, which indicates the size of the residual error. For larger residual errors the trace operation becomes large and increases the value of the adaptive factor. If the $R_{k}$ diagonal entries get larger the fading factor will increase thus forcing the filter to base its estimates on new data. Whereas if the $R_{k}$ matrix is close to zero the adaptive factor will be close to zero meaning that when convergence is achieved it bases its estimates more on past data.

\subsection{Monte Carlo Approximation}

Monte Carlo² (MC) algorithm is a stochastic sampling approach aiming to tackle the complex systems which are analytically intractable. The power of $M C$ technique is expressed in its ability to avoid numerical integration by mapping integrals into discrete sums. Much of the initial development of Monte Carlo occurred during World War II at Los Alamos National Laboratory, with the work of Stanislaw Ulam, Nicholas Metropolis, Enrico Fermi and John von Neumann [Metropolis 1987]. Ulam invented the method while working on nuclear weapon projects and Metropolis used the term Monte Carlo to describe analytical methods using the propagation of random samples to represent changes in probability distributions [Metropolis \& Ulam 1949]. Their contribution was central to the simulations required for the Manhattan Project, though severely limited by the computational tools at the time. The principle of Monte Carlo relies on the law of unconscious statistician ${ }^{3}$ where the expected value of a function $f_{k}(\cdot)$ of a random variable $\mathrm{x}_{0: k}$ with given probability

\footnotetext{
${ }^{2}$ The name was first suggested by Stanislaw Ulam to highlight a method named after a city in Monaco principally because of a roulette, a simple random number generator.

${ }^{3}$ The law of unconscious statistician is a theorem used to calculate the expected value of a function $f(\mathrm{x})$ of a random variable $\mathrm{x}$ when the probability distribution of $\mathrm{x}$ is known but the distribution of $f(\mathrm{x})$ is not known explicitly.
} 
distribution $p\left(\mathrm{x}_{0: k} \mid \mathrm{y}_{1: k}\right)$ is computed as follows

$$
\mathbb{E}\left(f_{k}\left(\mathrm{x}_{0: k}\right)\right)=\int f_{k}\left(\mathrm{x}_{0: k}\right) p\left(\mathrm{x}_{0: k} \mid \mathrm{y}_{1: k}\right) \mathrm{dx}_{0: k}
$$

The expected value in Equation (2.15) is approximated by avoiding the numerical integration and mapping the integral into a discrete sum as follows

$$
\mathbb{E}\left(f_{k}\left(\mathrm{x}_{0: k}\right)\right) \approx \frac{1}{N_{p}} \sum_{i=1}^{N_{p}} f_{k}\left(\mathrm{x}_{0: k}^{(i)}\right)
$$

where the samples $\mathrm{x}_{0: k}^{(i)}$ are assumed to be independent and identically distributed (i.i.d) support points for the approximation to hold. According to the law of large numbers, the approximation almost surely converges to the expectation as the number of support points $N_{p}$ increases.

\subsection{Bayesian Importance Sampling}

The objective of importance sampling is to sample the distribution in the region of importance in order to achieve computational efficiency. It is almost impossible to sample directly from the posterior density function in Equation (2.15); therefore, a proposal distribution $q\left(\mathrm{x}_{0: k} \mid \mathrm{y}_{1: k}\right)$ (see Appendix A for details) and a Bayesian algorithm are suggested to approximate the posterior distribution as follows:

$$
\mathbb{E}\left(f_{k}\left(\mathrm{x}_{0: k}\right)\right)=\int f_{k}\left(\mathrm{x}_{0: k}\right) \frac{p\left(\mathrm{x}_{0: k} \mid \mathrm{y}_{1: k}\right)}{q\left(\mathrm{x}_{0: k} \mid \mathrm{y}_{1: k}\right)} q\left(\mathrm{x}_{0: k} \mid \mathrm{y}_{1: k}\right) \mathrm{dx}_{0: k}
$$

Using Baye's rule

$$
\begin{aligned}
\mathbb{E}\left(f_{k}\left(\mathrm{x}_{0: k}\right)\right) & =\int f_{k}\left(\mathrm{x}_{0: k}\right) \frac{p\left(\mathrm{y}_{1: k} \mid \mathrm{x}_{0: k}\right) p\left(\mathrm{x}_{0: k}\right)}{p\left(\mathrm{y}_{1: k}\right) q\left(\mathrm{x}_{0: k} \mid \mathrm{y}_{1: k}\right)} q\left(\mathrm{x}_{0: k} \mid \mathrm{y}_{1: k}\right) \mathrm{dx} \mathrm{x}_{0: k} \\
& =\int f_{k}\left(\mathrm{x}_{0: k}\right) \frac{w_{k}\left(\mathrm{x}_{0: k}\right)}{p\left(\mathrm{y}_{1: k}\right)} q\left(\mathrm{x}_{0: k} \mid \mathrm{y}_{1: k}\right) \mathrm{dx}_{0: k}
\end{aligned}
$$

where the variable $w_{k}$ is the unnormalized importance weight

$$
w_{k}=\frac{p\left(\mathrm{y}_{1: k} \mid \mathrm{x}_{0: k}\right) p\left(\mathrm{x}_{0: k}\right)}{q\left(\mathrm{x}_{0: k} \mid \mathrm{y}_{1: k}\right)}
$$




\subsection{Bayesian Importance Sampling}

furthermore, isolating the normalizing density $p\left(\mathrm{y}_{1: k}\right)$ gives

$$
\mathbb{E}\left(f_{k}\left(\mathrm{x}_{0: k}\right)\right)=\frac{1}{p\left(\mathrm{y}_{1: k}\right)} \int f_{k}\left(\mathrm{x}_{0: k}\right) w_{k}\left(\mathrm{x}_{0: k}\right) q\left(\mathrm{x}_{0: k} \mid \mathrm{y}_{1: k}\right) \mathrm{dx}_{0: k}
$$

where

$$
\begin{aligned}
p\left(\mathrm{y}_{1: k}\right) & =\int p\left(\mathrm{y}_{1: k} \mid \mathrm{x}_{0: k}\right) p\left(\mathrm{x}_{0: k}\right) \frac{q\left(\mathrm{x}_{0: k} \mid \mathrm{y}_{1: k}\right)}{q\left(\mathrm{x}_{0: k} \mid \mathrm{y}_{1: k}\right)} \mathrm{dx}_{0: k} \\
& =\int w_{k}\left(\mathrm{x}_{0: k}\right) q\left(\mathrm{x}_{0: k} \mid \mathrm{y}_{1: k}\right) \mathrm{dx}_{0: k}
\end{aligned}
$$

therefore,

$$
\mathbb{E}\left(f_{k}\left(\mathrm{x}_{0: k}\right)\right)=\frac{\mathbb{E}_{q}\left(w_{k}\left(\mathrm{x}_{0: k}\right) f_{k}\left(\mathrm{x}_{0: k}\right)\right)}{\mathbb{E}_{q}\left(w_{k}\left(\mathrm{x}_{0: k}\right)\right)}
$$

where the notation $\mathbb{E}_{q}(\cdot)$ denotes expectation taken over the proposal distribution $q\left(\cdot \mid \mathrm{y}_{1: k}\right)$. Hence, by drawing samples from the proposal distribution the expectation can be approximates as follows

$$
\begin{aligned}
\mathbb{E}\left(f_{k}\left(\mathrm{x}_{0: k}\right)\right) & \approx \frac{\frac{1}{N_{p}} \sum_{i=1}^{N_{p}} f_{k}\left(\mathrm{x}_{0: k}^{(i)}\right) w_{k}\left(\mathrm{x}_{0: k}^{(i)}\right)}{\frac{1}{N_{p}} \sum_{i=1}^{N_{p}} w_{k}\left(\mathrm{x}_{0: k}^{(i)}\right)} \\
& \approx \sum_{i=1}^{N_{p}} f_{k}\left(\mathrm{x}_{0: k}^{(i)}\right) \tilde{w}_{k}\left(\mathrm{x}_{0: k}^{(i)}\right)
\end{aligned}
$$

where the normalized importance weight $\tilde{w}_{k}^{(i)}$ is given by

$$
\tilde{w}_{k}^{(i)}=\frac{w_{k}^{(i)}}{\sum_{j=1}^{N_{p}} w_{k}^{(j)}}
$$

thus as $N_{p}$ approaches infinity, the posterior density function can be approximated as the point-mass estimate

$$
p\left(\mathrm{x}_{0: k} \mid \mathrm{y}_{1: k}\right) \approx \sum_{i=1}^{N_{p}} \tilde{w}_{k}^{(i)} \delta\left(\mathrm{x}_{0: k}-\mathrm{x}_{0: k}^{(i)}\right)
$$

where $\delta(\cdot)$ denotes the Dirac delta function. Importance sampling, in its simplest form, is not adequate for sequential estimation since all the observations $\mathrm{y}_{1: k}$ must be known before estimating $p\left(\mathrm{x}_{0: k} \mid \mathrm{y}_{1: k}\right)$. Every time a new observation becomes available the importance sampling must be computed over the entire state sequence which increases computation complexity over time. 


\subsection{Particle Filter}

The Bayesian importance sampling method (detailed in Section 2.7), can be modified for sequential estimations. In sequential settings, the filter is called particle filter (PF) or sequential importance sampling (SIS). The various versions of particle filters proposed in literature can be regarded as special case of SIS algorithm. The details of the algorithm requires to characterize the posterior probability distribution $p\left(\mathrm{x}_{0: k} \mid \mathrm{y}_{1: k}\right)$ with a random measure $\left\{\mathrm{x}_{0: k}^{(i)}, w_{k}^{(i)}\right\}_{i=1}^{N_{p}}$, where $\left\{\mathrm{x}_{0: k}^{(i)}, i=0, \ldots, N_{p}\right\}$ are the state hypotheses with associated weights $\left\{w_{k}^{(i)}, i=0, \ldots, N_{p}\right\} . \mathrm{x}_{0: k}=\left\{\mathrm{x}_{j}, j=0, \ldots, k\right\}$ is a set of all states from $\mathrm{x}_{0}$ to $\mathbf{x}_{k}$. The weights are normalized to unity according to Equation (2.24) and the empirical posterior density at $k$ is approximated as the weighted sum of all samples evaluated at the dirac delta distribution given in Equation (2.25). Therefore, the true posterior density, $p\left(\mathrm{x}_{0: k} \mid \mathrm{y}_{1: k}\right)$, is expressed as a discrete weighted approximation to the true posterior density where the weights are chosen using the principle of importance sampling (see Appendix A).

Figure 2.3 shows the distribution of state hypotheses $\mathbf{x}_{k}^{(i)}$ and the corresponding probability mass $w_{k}^{(i)}$. Note that no specific assumption is made about the state distribution and the approximation improves as the number of support points increases since the MonteCarlo representation becomes an equivalent characterization of the posterior density.

The objective of sequential importance sampling (SIS) is to find samples to approximate the priori density at each iteration and approximate the posterior density with the new set of samples. The derivation of the posterior density update equation requires to express the posterior density in terms of priori density, likelihood density and state transition function (see Chapter 2.3) and using the Bayesian rule to express the posterior density as follows:

$$
\begin{aligned}
p\left(\mathrm{x}_{0: k} \mid \mathrm{y}_{1: k}\right) & =\frac{p\left(\mathrm{y}_{k} \mid \mathrm{x}_{0: k}, \mathrm{y}_{1: k-1}\right) p\left(\mathrm{x}_{0: k} \mid \mathrm{y}_{1: k-1}\right)}{p\left(\mathrm{y}_{k} \mid \mathrm{y}_{1: k-1}\right)} \\
& =\frac{p\left(\mathrm{y}_{k} \mid \mathrm{x}_{0: k}, \mathrm{y}_{1: k-1}\right) p\left(\mathrm{x}_{k} \mid \mathrm{x}_{0: k-1}, \mathrm{y}_{1: k-1}\right)}{p\left(\mathrm{y}_{k} \mid \mathrm{y}_{1: k-1}\right)} p\left(\mathrm{x}_{0: k-1} \mid \mathrm{y}_{1: k-1}\right) \\
& =\frac{p\left(\mathrm{y}_{k} \mid \mathrm{x}_{k}\right) p\left(\mathrm{x}_{k} \mid \mathrm{x}_{k-1}\right)}{p\left(\mathrm{y}_{k} \mid \mathrm{y}_{1: k-1}\right)} p\left(\mathrm{x}_{0: k-1} \mid \mathrm{y}_{1: k-1}\right) \\
& \propto p\left(\mathrm{y}_{k} \mid \mathrm{x}_{k}\right) p\left(\mathrm{x}_{k} \mid \mathrm{x}_{k-1}\right) p\left(\mathrm{x}_{0: k-1} \mid \mathrm{y}_{1: k-1}\right)
\end{aligned}
$$

Selecting the weights according the principle of importance sampling (see Chapter 2.7), the 


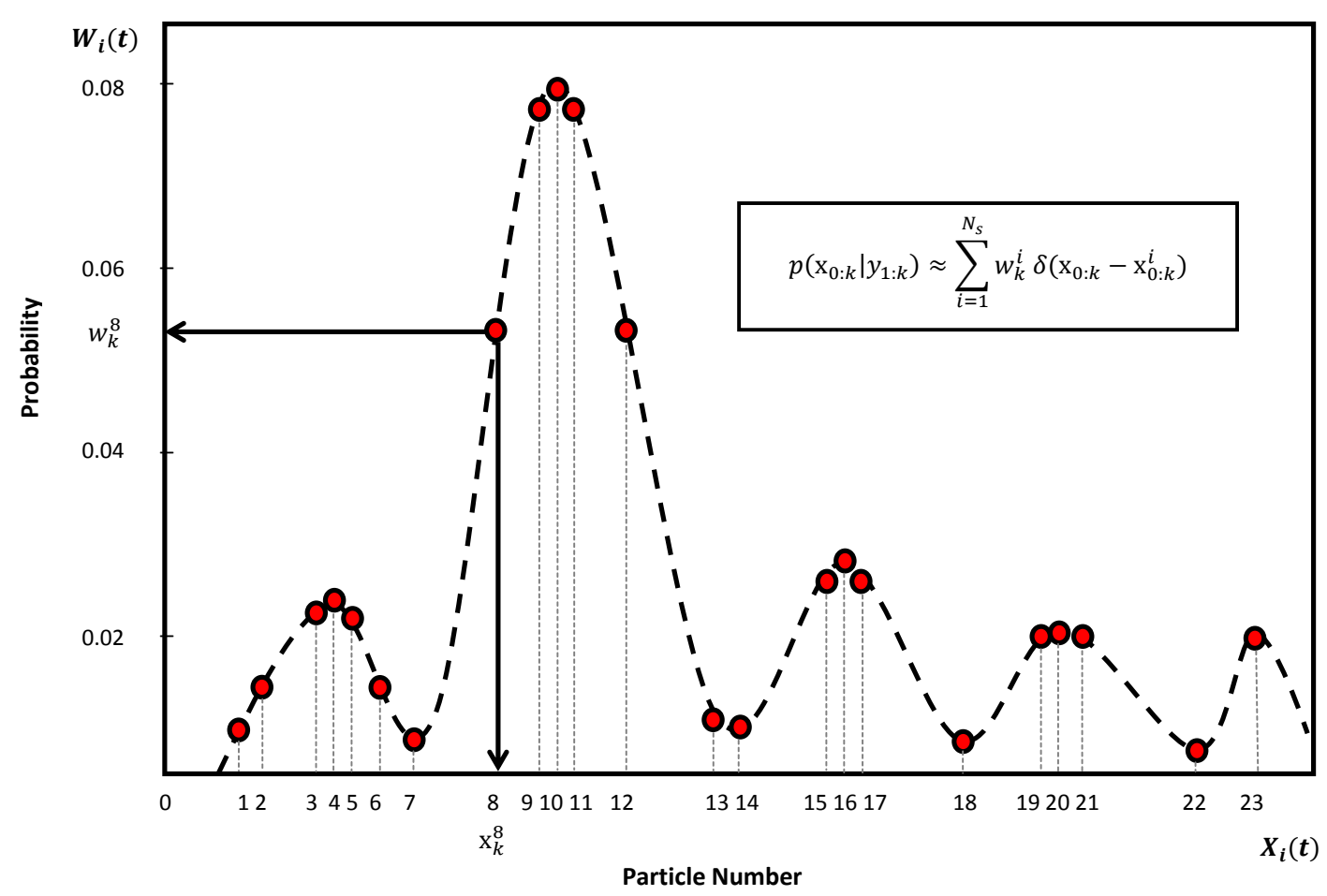

Figure 2.3: Particle filter representation of posterior distribution

weight is defined by

$$
w_{k}^{(i)} \propto \frac{p\left(\mathbf{x}_{0: k}^{(i)} \mid \mathbf{y}_{1: k}\right)}{q\left(\mathbf{x}_{0: k}^{(i)} \mid \mathbf{y}_{1: k}\right)}
$$

where $q\left(\mathbf{x}_{0: k}^{(i)} \mid \mathbf{y}_{1: k}\right)$ is the importance density from which the samples $\mathbf{x}_{0: k}^{(i)}$ are drawn from. It is possible to select an importance density such that

$$
q\left(\mathbf{x}_{0: k} \mid \mathbf{y}_{1: k}\right)=q\left(\mathbf{x}_{k} \mid \mathbf{x}_{0: k-1}, \mathbf{y}_{1: k}\right) q\left(\mathbf{x}_{0: k-1} \mid \mathbf{y}_{1: k-1}\right)
$$

The weight update equation is derived by substituting Equations (2.26) and (2.28) into $(2.27)$

$$
\begin{aligned}
w_{k}^{(i)} & \propto \frac{p\left(\mathbf{y}_{k} \mid \mathbf{x}_{k}^{(i)}\right) p\left(\mathbf{x}_{k}^{(i)} \mid \mathbf{x}_{k-1}^{(i)}\right) p\left(\mathbf{x}_{0: k-1}^{(i)} \mid \mathbf{y}_{1: k-1}\right)}{q\left(\mathbf{x}_{k}^{(i)} \mid \mathbf{x}_{0: k-1}^{(i)}, \mathbf{y}_{1: k}\right) q\left(\mathbf{x}_{0: k-1}^{(i)} \mid \mathbf{y}_{1: k-1}\right)} \\
& =w_{k-1}^{(i)} \frac{p\left(\mathbf{y}_{k} \mid \mathbf{x}_{k}^{(i)}\right) p\left(\mathbf{x}_{k}^{(i)} \mid \mathbf{x}_{k-1}^{(i)}\right)}{q\left(\mathbf{x}_{k}^{(i)} \mid \mathbf{x}_{0: k-1}^{(i)}, \mathbf{y}_{1: k}\right)}
\end{aligned}
$$

Equation (2.29) provides a mechanism for sequential update of the importance weights, given the appropriate choice of proposal distribution. Assuming a Markov process where 
$q\left(\mathbf{x}_{k} \mid \mathbf{x}_{0: k-1}, \mathbf{y}_{1: k}\right)=p\left(\mathbf{x}_{k} \mid \mathbf{x}_{k-1}, \mathbf{y}_{k}\right)$, the importance density dependent on $\mathbf{x}_{k-1}$ and $\mathbf{y}_{k}$. In this case, only $\mathbf{x}_{k}^{(i)}$ has to be stored whereas the path $\mathbf{x}_{0: k-1}^{(i)}$ and the history of observations $\mathbf{y}_{1: k-1}$ can be discarded. Finally, the posterior density $p\left(\mathbf{x}_{k} \mid \mathbf{y}_{1: k}\right)$ is approximated as

$$
p\left(\mathbf{x}_{k} \mid \mathbf{y}_{1: k}\right) \approx \sum_{i=1}^{N_{p}} w_{k}^{(i)} \delta\left(\mathbf{x}_{k}-\mathbf{x}_{k}^{(i)}\right)
$$

It can be shown that as the number of support points increases $N_{p} \rightarrow \infty$ the posterior density approximation in Equation (2.30) approaches the true posterior density $p\left(\mathbf{x}_{k} \mid \mathbf{y}_{1: k}\right)$.

SEQUENTIAL IMPORTANCE SAMPLING (SIS) [Särkkä 2013]

1. Draw $N_{p}$ samples from the prior distribution

$$
\mathbf{x}_{0}^{(i)} \sim p\left(\mathbf{x}_{0}\right), \quad i=1,2, \ldots, N_{p}
$$

2. Initialize the importance weight

$$
w_{0}^{(i)}=\frac{1}{N_{p}}, \quad i=1,2, \ldots, N_{p}
$$

3. For $k=1, \ldots, T$

(a) Draw samples from the importance distribution

$$
\mathbf{x}_{k}^{(i)} \sim q\left(\mathbf{x}_{k}^{(i)} \mid \mathbf{x}_{0: k-1}^{(i)}, \mathbf{y}_{1: k}\right), \quad i=1,2, \ldots, N_{p}
$$

(b) Calculate the updated importance weights

$$
w_{k}^{(i)}=w_{k-1}^{(i)} \frac{p\left(\mathbf{y}_{k} \mid \mathbf{x}_{k}^{(i)}\right) p\left(\mathbf{x}_{k}^{(i)} \mid \mathbf{x}_{k-1}^{(i)}\right)}{q\left(\mathbf{x}_{k}^{(i)} \mid \mathbf{x}_{0: k-1}^{(i)}, \mathbf{y}_{1: k}\right)}, \quad i=1,2, \ldots, N_{p}
$$

4. Normalize the importance weight such that $\sum_{i}^{N_{p}} \tilde{w}_{k}^{(i)}=1$

$$
\tilde{w}_{k}^{(i)}=\frac{w_{k}^{(i)}}{\sum_{j=1}^{N_{p}} w_{k}^{(j)}}
$$




\subsection{Particle Filter}

5. Approximate the posterior distribution

$$
p\left(\mathrm{x}_{0: k} \mid \mathrm{y}_{1: k}\right) \approx \sum_{i=1}^{N_{p}} \tilde{w}_{k}^{(i)} \delta\left(\mathrm{x}_{0: k}-\mathrm{x}_{0: k}^{(i)}\right)
$$

The problem with SIS algorithm is that as $k$ increase, the distribution of the importance weight $\tilde{w}_{k}^{(i)}$ becomes more skewed. After few iterations all the particles have a zero or nearly zero weight and the algorithm fails to adequately represent the posterior distribution. This is called the degeneracy problem.

Degeneracy Problem: The particle filter suffers from degeneracy problem where after few iterations most of the particles except for a very few will have a insignificant weight. Degeneracy implies that a large computational effort is devoted to updating particles whose contribution to the posterior approximation $p\left(\mathrm{x}_{k} \mid \mathrm{y}_{1: k}\right)$ is negligible. The degeneracy problem is an undesirable effect in particle filter and since the variance of the importance weight only increases over time, it is impossible to void the phenomenon. A well-know measure of degeneracy of the filter is the effective sample size $N_{\text {eff }}$ defined as [Arnaud Doucet 2001], [Liu 2008]

$$
N_{e f f}=\frac{N_{p}}{1+\operatorname{Var}\left(w_{k}^{* i}\right)}
$$

where $w_{k}^{* i}$ is the "true" weight.

$$
w_{k}^{* i}=p\left(\mathrm{x}_{k}^{i} \mid \mathrm{y}_{1: k}\right) / q\left(\mathrm{x}_{k}^{i} \mid \mathrm{x}_{k-1}^{i}, \mathrm{y}_{k}\right)
$$

This can not be evaluated exactly, but an estimate $\hat{N}_{\text {eff }}$ of $N_{\text {eff }}$ can be obtained by

$$
\hat{N}_{e f f}=\frac{1}{\sum_{i=1}^{N_{p}}\left(w_{k}^{i}\right)^{2}}
$$

where $w_{k}^{i}$ is the normalized weight in Equation (2.24). Notice that $N_{\text {eff }} \leq N_{p}$, and small $N_{\text {eff }}$ implies sever degeneracy. One way of reducing the degeneracy phenomenon is to use very large sample size. However, a very large sample size with a fixed number of particles results in approximation with low computational efficiency, especially when the true posterior changes over time [Wang \& Chaib-draa 2012]. Several methods have been introduced to prevent the degeneracy problem[Arnaud Doucet 2001],[Robert \& Casella 2005]. Simple 
approaches to solve the degeneracy problem include: (1) selecting an optimal importance density and (2) use a resampling strategy.

Resampling: The Sequential Importance Sampling (SIS) algorithm suffers from degeneracy problem where after few iterations all but one particle will have a negligible weight. The usual strategy around this is to introduce a resampling step. The resampling step is a crucial and computationally expensive part of SIS algorithm. With resampling, the particles with large weights are replicated and the ones with negligible weights are removed. After resampling, the future particles are more concentrated in domains of higher posterior probability, which entails improved estimates.

The scientific literature on resampling identifies mainly four resampling techniques in a particle filter setting. These include the multinomial, residual, stratified and systematic resampling algorithms. Resampling at every iteration is not necessary for on-line state estimation since the resampling state consume computation power and may degrade the efficiency of the algorithm. It is thus desired to prescribe a schedule for the resampling step to take place [Liu \& Chen 1998]. Two resampling schedules are possible: (1) deterministic resampling schedular where the resampling is performed at fixed intervals $k, 2 k, \ldots, T$. (2) dynamic resampling schedular where a sequence of threshold are defined and the coefficient of variations is monitored. A detailed discussion on these specific resampling algorithm will be provided in the following chapters. The aim of resampling is to replace the prior set of $N_{p}$ particles by a new set of particles, where the particles have been duplicated or rejected according to their corresponding weight. The higher the weight of a particle, the greater the chance that the particle will be drawn several times. A well argued choice of resampling method is justified as the entire estimation process benefit from reduced complexity in the resampling step. Different variations of particle filters were developed and special cases can be derived from the SIS algorithm by appropriate choice of importance sampling and modification of the resampling step.

An investigation and detailed discussions on selection process of importance sampling and various resampling techniques are provided in the following chapters. 


\subsection{Chebyshev Neural Networks}

\subsection{Chebyshev Neural Networks}

Chebyshev neural networks (CNN) have been implemented to conquer difficulties with unknown nonlinearity and time-varying characteristics of many nonlinear dynamic systems. Basios et al. [Basios et al. 1997] have approximated a one-dimensional function defined on a discrete set of points using orthogonal first-order Chebyshev polynomials and artificial feed-forward neural network. Lee and Jeng [Lee \& Jeng 1998] proposed an approximate transformable technique to obtain a Chebyshev polynomials based (CPB) unified model neural networks for feedforward/recurrent neural networks via Chebyshev polynomials approximation. Based on this approximate transformable technique, Lee and Jeng have derived a relationship between a single-layer neural networks and multilayer perceptron (MLP) neural networks based on Chebyshev polynomial model. It is pointed out that this network has universal approximation capability and has faster convergence than the multilayer feed-forward neural network. Akritas [Akritas et al. 2000] have applied CNN to reconstruct and predict a discrete chaotic maps. The method is based on feed-forward neural network which decomposes the analyzed chaotic map in orthogonal Chebyshev polynomials. A single-layer functional link neural network in which the need of hidden layer is eliminated by expanding the input pattern by Chebyshev polynomials is proposed for the purpose of dynamic nonlinear system identification [Patra \& Kot 2002]. System identification schemes for unknown dynamic nonlinear discrete time system has also been proposed using CNN in a feed-forward neural network framework that ensures identification of general nonlinear dynamical systems with smooth nonlinearities [Purwar et al. 2007]. Second-order nonlinear ordinary differential equations of Lane-Emden type was solved using feed-forward CNN model and principle of error back propagation [Mall \& Chakraverty 2014]. In this work, Mall implemented a single layer neural network to eliminate the hidden layer by expanding the input pattern by Chebyshev polynomials. An adaptive recurrent CNN control system was also proposed to control a permanent magnet synchronous motor (PMSM) servo-drive electric scooter with V-belt CVT under lumped nonlinear external disturbances [Lin 2015]. Essentially, learning a nonlinear function boils down to obtaining a description of the conditional distribution through application of a mapping function. When the exact structure 
of this mapping cannot be established a priori, it may be synthesized as a combination of parameterized basis functions and an estimated system weights. Essentially, the unknown function can be approximated in a classical neural networks scheme as follows:

Theorem 1 [Poggio \& Girosi 1990]:

A continuous function $f(\mathrm{x})$ can be approximated as the product of a basis function and associated weight

$$
\hat{f}(\mathrm{x})=W \xi(\mathrm{x})+\varepsilon
$$

where $\hat{f}(\mathrm{x})$ is the approximation of the continuous function, $\xi(\mathrm{x})$ is the basis function with associated weight $W, \varepsilon$ is the approximation error, and $\mathrm{x}$ is the state vector over a compact set.

Definition [Poggio \& Girosi 1990]:

The quality of the approximation can be measured by a distance function

$$
\rho=|\hat{f}(\mathrm{x})-f(\mathrm{x})|
$$

where $\rho$ determines the distance between the approximated function $\hat{f}(\mathrm{x})$, and the continuous function $f(\mathrm{x})$.

Problem statement [Poggio \& Girosi 1990]:

If $f(\mathrm{x})$ is a continuous function defined on set $\mathrm{x}$, and $\hat{f}(\mathrm{x})$ is an approximating function that depends continuously on $W$ and $\mathrm{x}$, the approximation problem is to determine the parameters $W^{*}$ such that

$$
\rho\left[\hat{f}\left(\mathrm{x}, W^{*}\right), f(\mathrm{x})\right] \leq \rho[\hat{f}(\mathrm{x}, W), f(\mathrm{x})]
$$

for all $W$ in the set $P$, and $W^{*} \in R^{m \times N_{1}}$ is the optimal weight matrix with $N_{1}=2 m N_{2}+1$ and $N_{2}$ is the order of the Chebyshev polynomials.

Several methods have been developed in constructing the basis functions $\xi(\mathrm{x})$ in Equation (2.39) i.e., linear mapping, sigmoid function etc. Here, the attention is directed 


\subsection{Concluding Remarks}

to Chebyshev polynomial functions. The Chebyshev polynomial basis function $\xi\left(X_{i}\right)$ $(i=1,2, \cdots, n)$ is obtained by Chebyshev polynomial as follows

$$
\xi_{i}\left(X_{i}\right)=\left(1, T_{1}\left(X_{i, 1}\right), \cdots, T_{N_{2}}\left(X_{i, 1}\right), \cdots, T_{1}\left(X_{i, 2 m}\right), \cdots, T_{N_{2}}\left(X_{i, 2 m}\right)\right)^{T}
$$

where $T_{i}\left(X_{i, j}\right)\left(i=1,2, \cdots, N_{2}, j=1,2, \cdots, 2 m\right)$ represent Chebyshev polynomials, which are a set of orthogonal polynomials derived from the solution of Chebyshev differential equations and obtained using the two-term recursive formula as follows

$$
\begin{aligned}
T_{0}\left(X_{i, j}\right) & =1 \\
T_{1}\left(X_{i, j}\right) & =X_{i, j} \\
T_{i+1}\left(X_{i, j}\right) & =2 X_{i, j} T_{i}\left(X_{i, j}\right)-T_{i-1}\left(X_{i, j}\right)
\end{aligned}
$$

where $T_{0}\left(X_{i, j}\right)$ and $T_{1}\left(X_{i, j}\right)$ are the initial conditions of CNN.

\subsection{Concluding Remarks}

Several approaches were developed to deal with nonlinear filtering which attempt to extract state estimations from noisy measurements. Throughout this dissertation, an emphasis is placed on studying two nonlinear filters which are the adaptive unscented Kalman filter (AUKF) and particle filter (PF). To understand, the development of these filters a background was needed in Bayesian algorithm which is covered in section 2.3. Section 2.4 covers the unscented Kalman filter (UKF) which provide a better nonlinear state approximation with reduced error as compared to KF or EKF. The unscented Kalman filter is an extension to Kalman filter which takes distinctly different approach to the nonlinear Gaussian problem. In this approach, a statistical method is developed where deterministic sigma points are transformed to a new nonlinear space and then statistics are calculated to obtain the required state approximation. Section 2.8 covers the sequential importance sampling (SIS) algorithm for state estimation. The SIS algorithm (also known as Sequential Monte Carlo (SMC) or particle filter) forms the basis for most sequential filters. Indeed, it has become traditional to present particle filtering and SMC as being the same thing in much of the literature although SMC encompasses a broader range of algorithms. Finally in Section 2.9, Chebyshev neural networks (CNN) have been introduced. 


\section{CHAPter 3}

\section{Attitude Estimation and Control of}

\section{Monolithic Spacecraft}

The demand for high resolution monolithic spacecraft missions requires to develop an accurate and stable online attitude estimation and control techniques in order to increase the pointing capability of the spacecraft. The attitude estimation requires a simultaneous input from multiple sensors that measure the orientation of the spacecraft relative to a reference frame. The attitude must be estimated since no sensor exist to measure the attitude directly and it must be estimated from available noisy measurements. Here, the spacecraft is controlled in the absence of angular velocity measurements where the adaptive unscented Kalman filter and particle filter are utilized to estimate the quaternion and angular velocities. The attention in this chapter is directed to (1) online estimation of spacecraft attitude and angular velocities using the adaptive unscented Kalman filter (AUKF) and particle filer (PF) techniques, and (2) investigate the behavior of the AUKF and PF in the presence of faults at the spacecraft dynamics, control law, or sensor measurements. The nonlinear filtering techniques in this chapter were selected since the spacecraft attitude problem is nonlinear and an approach is needed to estimates the spacecraft attitude with nonlinear filters. The chapter is organized as follows: Section 3.1 provides a brief introduction to the attitude estimation problem which includes a short overview of various techniques used to solve the problem. Section 3.2 presents the equation of motion of a monolithic spacecraft which includes the kinematic and dynamic equation in quaternion presentation. Section 3.3 provides attitude estimation results for stabilization of a monolithic spacecraft. 


\subsection{Introduction}

\subsection{Introduction}

The orientation of a monolithic spacecraft with respect to a frame of reference is called the attitude of the spacecraft and the associated motion is the attitude motion. The spacecraft attitude must be estimated and precisely controlled in order to achieve an accurate Earth pointing capability. The attitude of a spacecraft is defined by two distinct coordinate frames: The body-fixed coordinate frame, which is attached to the spacecraft at its center of mass, and the reference frame, which consist of an abstract coordinate system attached to Earth, Sun, Moon, or other stars. The orientation of the body-fixed frame with respect to a reference frame determines the attitude of the spacecraft.

The attitude of the spacecraft is represented by three parameters where each parameter has a singularity or discontinuity for certain attitudes ${ }^{1}$. To overcome this singularity, a higher-dimensional nonsingular parameterization technique was developed with one extra degree of freedom which enforces a constraint and increases the complexity of representation. This nonsingular parameter is known as the four-component quaternion representation for attitude.

In this research, the four parameter quaternion and the spacecraft angular velocities are estimated using the adaptive unscented Kalman filter and particle filter. The spacecraft attitude and corresponding motion must be estimated since it is impossible to measure directly the exact state of the spacecraft and it must be estimated from noisy measurements.

The proposed nonlinear filtering techniques are part of the computer program that receive sequential data from the spacecraft measurement models and compute appropriate state estimations. The state estimations are sent to the control law which control the spacecraft actuators and position the spacecraft in desired attitude. The filter requires to determine the posterior probability density of a given dynamic system at each time instant, conditioned on the measurements available up to that time.

\footnotetext{
${ }^{1}$ Practical techniques to represent the spacecraft attitude include the direction cosines, Rodrigues parameters, rotation vectors, Euler angles, and Gibbs vector. Each of these techniques has its own advantages and disadvantages.
} 


\subsubsection{Literature review}

The Kalman filtering technique has been widely used for online spacecraft attitude estimation and control problems. The earliest published reference for attitude estimation is the work of J.L. Farrell [Farrell 1970] who studied the extent to which Kalman filtering of crude attitude measurements from Sun sensors and magnetometers could provide attitude accuracy equivalent to that obtained without smoothing from more elaborate instrumentation. Farrell represented the attitude by Euler angles and assumed torque free motion in the attitude prediction for both spinning and non-spinning rigid spacecraft. In the reported work, recognizable nonlinear effects could not be accurately estimated using Kalman filter.

Review of Kalman filter for attitude estimation is given by Lefferts [Lefferts et al. 1982] who reviewed the developments of suitable algorithms for spacecraft equipped with threeaxis gyros and line-of-sight attitude sensors. Throughout the report the attitude was presented by quaternion because the representation is free from singularities which prevents gimbal-lock situation. Another short review of application and development of Kalman filter techniques in the aerospace industry is provided in [Grewal S. Mohinder 2010].

Another reference work on attitude estimation using Kalman filtering technique is the work by Psiaki et. al. [Mark L. Psiaki 1990] who estimated the attitude, attitude rates and constant disturbance torques for a three-axis stabilized spacecraft using Kalman filter. Since a single three-axis magnetometer measurement provide only two-axes worth of attitude information and no attitude rate or disturbance torque information, Psiaki, proposed an attitude determination system that use a sequence of magnetometer measurements which are processed recursively in a Kalman filter setting.

A recent technical report from the Department of Computer Science \& Engineering at the University of Minnesota, Trawny and Roumeliotis [Nikolas Trawny 2005] created an extensive tutorial for quaternion algebra. In the report, Trawny and Roumeliotis used a Kalman Filter to estimate the attitude of a spacecraft using a quaternion representation. In their work, Trawny and Roumeliotis used an alternative approach to estimate position and orientation of the spacecraft by using data from an inertial measurement unit (IMU) as dynamic model replacement ${ }^{2}$.

\footnotetext{
${ }^{2}$ The IMU is an electrical device that measures and reports the spacecraft translational accelerations
} 


\subsection{Introduction}

The Kalman filter was originally developed as a tool for linear estimation and could not be applied directly to spacecraft attitude estimation given the nonlinear nature of the system dynamics. When the Kalman filter has been applied to nonlinear system it generated reasonable estimation in localized settings but failed to converge in nonlinear problems. Therefore, nonlinear theory was needed to encompass estimation of nonlinear dynamic system. The extended Kalman filter (EKF) has been successfully applied to nonlinear estimations and is the most widely used nonlinear filtering technique for attitude estimation.

Vathsal [Vathsal 1987] presented a second-order filter using EKF technique for estimating the attitude quaternion using three-axis gyro and star-tracker measurement data. In his work, Vathsal expended the system and measurement models to second-order using Taylor series approximation and compared the quaternion estimation from the proposed filter to the performance of EKF. Vathsal concluded that the EKF worked well in the linear regime where the linear approximation of the nonlinear dynamic system is valid. However, the filter failed to converge when the system dynamics is highly nonlinear.

Modern research in attitude estimation is based on unscented Kalman filter (UKF) and particle filter (PF) scheme. Xiong [Xiong K. 2007] introduced a fault detection method for nonlinear systems using the residual generated by unscented Kalman filter. In the study, Xiong introduced a detection algorithm for satellite attitude sensor faults using the UKF and a local approach for detecting changes in the mean of a Gaussian process where the heading of the spacecraft is computed by the roll, pitch, and yaw representation. Xiong introduced faults in the Sun sensor, Earth sensor, the Gyroscope, and incipient faults in the Sun sensor.

Rapoport and Oshman [Rapoport \& Oshman 2004] studied fault tolerant filtering problem of system with nonlinear dynamics and fault-prone measurements channels. Their work is based on particle filtering approach for estimating the angular velocity of a spacecraft and fault parameters of the measurement channel using four fault-prone rate gyros. The system under investigation included the overall state vector with both system states and the parameters of the measurements channels. Since the size of the state vector can be

and rotational velocities using a combination of three-axis accelerometers and gyroscopes. 
prohibitively large for efficient application of ordinary particle filter Rapoport and Oshman used the Row-Blackwellization technique which incorporate the interacting multiple model (IMM) algorithm. The reason for adopting the Row-Blackwellization technique is to bring the estimate the main system states to a reduced-size set of particles. In the work, the rate gyros were assumed to suffer from measurement noise and suddenly appearing and disappearing measurements biases that changed their values each time they appeared. To simplify the analysis it was assumed that the spacecraft is torque-free and no internal or external torques were acting on the spacecraft.

Cheng and Crassidis [Cheng \& Crassidis 2004] investigated spacecraft attitude estimation using the particle filter technique. The filter formulation, in their work, is based on star camera measurements using gyro-based or attitude dynamic-based model for attitude propagation. Cheng and Crassidis used the Modified Rodrigues parameters for attitude parametrization to compute the samples mean and covariance of the spacecraft attitude. Their proposed work is primarily based on attitude estimation using the Bootstrap filter, which was proposed by Gordon, Salmond and Smith [Gordon \& Smith 1993]. Cheng and Crassidis suggested to use a uniform attitude probability distribution as the initial attitude distribution and using gradually decreasing measurement variance in the computation of the importance weights for the particle filter based attitude estimator to obtain global convergence properties. In addition, they suggested to use as few particles as possible in the bootstrap filter since the computational cost of the filter is largely proportional to the number of particles. For the filter with a modest number of particles to work properly the sampling efficiently has to be enhanced. The work was concluded by stating that for large initial errors the bootstrap filter has much better convergence properties than the unscented Kalman filter because the bootstrap filter implicitly captures higher order characteristics of the posterior distribution. 


\subsection{Introduction}

\subsubsection{Objectives}

In the open literature, there has not been much emphasis on attitude estimation and control in an online setting under the presence of faults and without angular velocity measurements where the attitude is represented by the quaternion. The attitude estimation is based on dynamic models and measurement models where a recursive algorithm provides a sequential approach to combine the inputs of both models as they become available to estimate the attitude and angular velocity of the spacecraft. Here, the attitude and angular velocity are estimated by AUKF or particle filter. Since, the estimation process is done online, a time-delay is introduced in the estimation algorithm to incorporate the estimation delay between sequential measurements. The main contributions of this research are as follows:

1. Filter models: Two filter models are constructed to estimate the full state of a monolithic spacecraft in an online setting. The full state vector includes attitude and angular velocity estimations. The attitude states and angular velocities of the spacecraft are estimated using nonlinear filtering techniques.

2. Online estimation and control: Online estimation and control algorithms for monolithic spacecraft requires a sequential update of attitude states when new measurement becomes available. Here, the attitude and angular velocity are estimated using the adaptive unscented Kalman filter (AUKF) and particle filter (PF).

3. Fault scenarios: The proposed nonlinear filters are tested under abrupt, transient, intermittent and incipient fault cases. The faults are introduced at the spacecraft dynamic model, the applied control command, and measurement model. The faults are applied at different time intervals in an attempt to investigate the filter performance.

4. Performance evaluation: Performance evaluation and comparison between AUKF and PF. Here we challenge the claim by Cheng and Crassidis which states that for large initial errors the bootstrap filter (also known as particle filter) has much better convergence properties than UKF [Cheng \& Crassidis 2004]. The investigation focuses on calculations of quaternion error and angular velocity residuals and measured by the matrix of a root mean squared error. 
Chapter 3. Attitude Estimation and Control of Monolithic Spacecraft

\subsection{System Model, Filter Model, and Control Law}

The investigation of attitude estimation of monolithic spacecraft starts by defining the equation of motion of the spacecraft. Section 3.2.1 describes the dynamic equation of monolithic spacecraft, and Section 3.2.2 describes the kinematic equations.

\subsubsection{Dynamic equation}

The dynamic equation of a monolithic rigid spacecraft is given by [Hughes 1986]

$$
J \dot{\omega}=-\omega^{\times} J \omega+\tau+\vartheta
$$

where $\omega=\left[\omega_{1}, \omega_{2}, \omega_{3}\right]^{T}$ is the angular velocity vector of the spacecraft with respect to an inertial frame $\mathbb{I}$ and expressed in the body frame $\mathbb{B}, J \in R^{3 \times 3}$ is the positive-definite mass moment of inertia matrix, $\tau \in R^{3}$ is the applied control torque generated by actuators, $\vartheta$ is the external disturbance, and $x^{\times} \in R^{3 \times 3}$ for a vector $x=\left[x_{1}, x_{2}, x_{3}\right]^{T}$ denotes a skew-symmetric matrix given by

$$
x^{\times}=\left[\begin{array}{ccc}
0 & -x_{3} & x_{2} \\
x_{3} & 0 & -x_{1} \\
-x_{2} & x_{1} & 0
\end{array}\right]
$$

\subsubsection{Kinematic equations}

The kinematic equations of a monolithic spacecraft are given by [Hughes 1986]

$$
\dot{q}=\frac{1}{2}\left[\begin{array}{c}
q_{4} I_{3}+\bar{q}^{\times} \\
-\bar{q}^{T}
\end{array}\right] \omega=\frac{1}{2} A(q) \omega
$$

where $\bar{q}=\left[q_{1}, q_{2}, q_{3}\right]^{T}$, and the unit quaternion $q=\left[\bar{q}^{T}, q_{4}\right]^{T}=\left[q_{1}, q_{2}, q_{3}, q_{4}\right]^{T}$ represents the attitude of a monolithic rigid spacecraft in the body frame $\mathbb{B}$ with respect to the inertial frame $\mathbb{I}$, which is defined by

$$
q=\left[\begin{array}{c}
\bar{\varepsilon} \sin \left(\varepsilon_{0} / 2\right) \\
\cos \left(\varepsilon_{0} / 2\right)
\end{array}\right]=\left[\begin{array}{c}
\bar{q} \\
q_{4}
\end{array}\right]
$$




\subsection{System Model, Filter Model, and Control Law}

where $\bar{\varepsilon}=\left[\varepsilon_{1}, \varepsilon_{2}, \varepsilon_{3}\right]^{T}$ denotes the principal axis, and $\varepsilon_{0}$ is the principal angle. The unit quaternion $q$ satisfies the constraint $\|q\|=1$, and $q^{\times} \in R^{3 \times 3}$ denotes a skew-symmetric matrix. Let $q$ denote a given attitude, then $-q$ denote the same attitude after a rotation of $\pm 2 \pi$ about an arbitrary axis. The inverse of a quaternion $q$ is given by $q^{-1}=\left[-\bar{q}^{T}, q_{4}\right]^{T}$, and the quaternion error between $q$ and $\hat{q}$ is denoted as $\tilde{q}=\hat{q}^{-1} \odot q$ where $q \odot q_{o}$ is a quaternion multiplication defined as [Shuster 1993]

$$
q \odot q_{o}=\left[\begin{array}{c}
q_{4} \bar{q}_{o}+q_{o 4} \bar{q}+\bar{q}^{\times} \bar{q}_{o} \\
q_{4} q_{o 4}-\bar{q}^{T} \bar{q}_{o}
\end{array}\right]
$$

\subsubsection{Filter models}

The filter model for estimating the state of a monolithic rigid spacecraft is given by

$$
\hat{\mathrm{x}}_{k+1}=f\left(\hat{\mathrm{x}}_{k}, \tau_{k}\right)+w_{k}
$$

where $w_{k}$ is the process noise, $\tau_{k}$ is the control torque, and $\hat{\mathrm{x}}_{k}$ is the seven elements state vector, which is comprised of three angular velocities and four attitude motions represented in quaternion ${ }^{3}$. The notation $(\hat{\imath})$ denotes the estimation of $(\cdot)$. The augmented state estimation vector is given by $\hat{\mathrm{x}}_{k}=\left[\hat{\omega}_{k}, \hat{\bar{q}}_{k}, \hat{q}_{4 k}\right]^{T}$, and $f\left(\hat{\mathrm{x}}_{k}, \tau\right)$ is propagated through the forth-order Runge-Kutta method as follows,

$$
f\left(\hat{\mathrm{x}}_{k}, \tau\right)=\hat{\mathrm{x}}_{k}+\frac{h}{6}\left(K_{1}+K_{2}+K_{3}+K_{4}\right)
$$

where for a given time step, $h>0$, we define $t_{k+1}=t_{k}+h$, and

$$
\begin{aligned}
K_{1} & =G\left(t_{k}, \hat{\mathrm{x}}_{k}, \tau\right) \\
K_{2} & =G\left(t_{k}+\frac{h}{2}, \hat{\mathrm{x}}_{k}+\frac{h}{2} K_{1}, \tau\right) \\
K_{3} & =G\left(t_{k}+\frac{h}{2}, \hat{\mathrm{x}}_{k}+\frac{h}{2} K_{2}, \tau\right) \\
K_{4} & =G\left(t_{k}+h, \hat{\mathrm{x}}_{k}+h K_{3}, \tau\right)
\end{aligned}
$$

\footnotetext{
${ }^{3}$ The quaternion $\hat{q}_{4}$ does not have to be estimated since it can be directly computed from the quaternion constraint $\hat{q}_{1}^{2}+\hat{q}_{2}^{2}+\hat{q}_{3}^{2}+\hat{q}_{4}^{2}=1$. Generally, $\bar{q}(t) \rightarrow 0$ as $t \rightarrow \infty$, which implies $\hat{q}_{4} \rightarrow+1$ or $\hat{q}_{4} \rightarrow-1$. Because $q_{4}=-1$ is unstable equilibrium point, it is obtained that $q_{4} \rightarrow+1$ as $t \rightarrow \infty$ [Jin \& Sun 2008].
} 
and the function $G(\hat{\mathrm{x}})$ is given as

$$
G(\hat{\mathrm{x}})=\left[\begin{array}{c}
J_{m}^{-1}\left(-\hat{\omega}^{\times} J_{m} \hat{\omega}+\tau\right) \\
\frac{1}{2} A(q) \hat{\omega}
\end{array}\right]
$$

$\hat{\omega}$ is estimated angular velocity vector, $J_{m} \in R^{3 \times 3}$ is the positive-definite mass moment of inertia matrix of the filter model, and $\hat{\omega}^{\times} \in R^{3 \times 3}$ is the skew-symmetric matrix. $A(q)$ is given as

$$
A(q)=\left[\begin{array}{c}
q_{4} I_{3}+\bar{q}^{\times} \\
-\bar{q}^{T}
\end{array}\right]
$$

where $\bar{q}$ is obtained directly from the measurement model $y_{k}$ which is given by

$$
y_{k}=\left[\bar{q}_{k}\right]+\left[v_{q k}\right]
$$

where $y_{k} \in \mathbb{R}^{3}$ are the measurements, $\bar{q}_{k}$ is the quaternion, and $v_{q k}$ is the zero-mean additive noise. Please note that $q_{4}$ is computed from the quaternion constraint $q_{1}^{2}+q_{2}^{2}+q_{3}^{2}+q_{4}^{2}=1$, and $q^{\times} \in R^{3 \times 3}$ denotes a skew-symmetric matrix,

\subsubsection{Control law}

For controlling a monolithic spacecraft without angular velocity measurements, the following asymptotic attitude control law was selected [Zou et al. 2014]

$$
\tau=J_{m}\left[-k_{2} \eta+J_{m}^{-1} \hat{\omega}^{\times} J \hat{\omega}-\theta^{2} \gamma \overline{\tilde{q}}\right]
$$

where $\hat{\omega}$ is the estimated angular velocity vector, $J_{m}$ is the moment of inertia matrix, $k_{1}$, $k_{2}, \gamma$ and $\theta$ are tunable control parameters, $\eta=\hat{\omega}-\hat{\omega}_{d}$, where $\hat{\omega}_{d}=-k_{1} \bar{q}$, and $\tilde{q}=\hat{q}^{-1} \odot q$ is the quaternion error between $q$ and the estimate $\hat{q}$. Note that the control law is applied to the system dynamics using sample and hold approach. 


\subsection{Simulation Results and Discussion}

\subsection{Simulation Results and Discussion}

The following section provides simulation results and discussion on fault-free and faultprone scenarios of a monolithic spacecraft. The forth-order Runge-Kutta method was implemented to numerically integrate the system model and filter model with an integration time step $t=0.01 \mathrm{sec}$ and $t=0.01 \mathrm{sec}$ for a simulation time of $T_{\text {sim }}=600 \mathrm{sec}$. The desired attitude trajectory is considered as follows, $q_{d}=[0,0,0,1]$.

System parameters: The initial conditions of the states are set as: $\bar{q}(0)=$ $[-0.6,0.4,-0.2]^{T}, q_{4}(0)=\sqrt{1-\|\bar{q}(0)\|^{2}}$, and $\omega(0)=[1,1,1]^{T}$, the spacecraft mass mo-

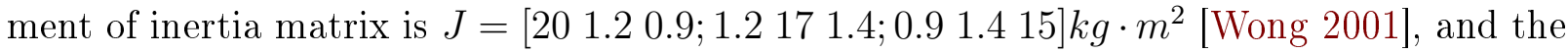
external disturbances vector is given by $\vartheta=d_{k}[\sin (t), \cos (t), \sin (2 t)]^{T}$, where $d_{k}=0.1$.

Control parameters: The saturation limit of the control law is $\left|\tau_{i}\right| \leq 0.1 \mathrm{Nm}$, for ( $i=$ $1,2,3)$, the control parameters $\nu=1, k_{1}=0.05, k_{2}=15, \gamma=2, \theta=10$, and the spacecraft moment of inertia matrix is $J_{m}=[2000 ; 0170 ; 0015] \mathrm{kg} \cdot \mathrm{m}^{2}$.

Filter parameters: The initial conditions are: $\hat{\omega}(0)=[0,0,0]^{T}, \hat{q}_{1}(0)=-0.6$, $\hat{q}_{2}(0)=0.4, \hat{q}_{3}(0)=-0.2$, and $\hat{q}_{4}(0)=\sqrt{1-\|\bar{q}(0)\|^{2}}$. The filter moment of inertia is $J_{m}=[2000 ; 0170 ; 0015] \mathrm{kg} \cdot \mathrm{m}^{2}$. The standard deviation of process noise is $\sigma_{Q}=1 \times 10^{-3}$, and the standard deviation of measurement noise is $\sigma_{R}=1 \times 10^{-4}$. The covariance matrix of process noise is given by $Q=E\left\{w w^{T}\right\}=\sigma_{Q}^{2} I_{n \times n}$, and the covariance matrix of measurement noise is given by $R=E\left\{v v^{T}\right\}=\sigma_{R}^{2} I_{m \times m}$.

Adaptive Unscented Kalman Filter: The scaling parameter $\lambda=\left(\alpha_{A U K F}\right)^{2} \times(n+\kappa)-n$, where $\alpha_{A U K F}=0.1, \beta_{A U K F}=2, \kappa=0$, and $n$ is the dimension of the augmented state. The filter weights are $\mathrm{W}_{0}^{(m)}=\lambda /(n+\lambda), \mathrm{W}_{0}^{(c)}=\lambda /(n+\lambda)+\left(1-\alpha_{A U K F}^{2}+\beta_{A U K F}\right)$, and $\mathrm{W}_{i}^{(m)}=$ $\mathrm{W}_{i}^{(c)}=1 / 2(n+\lambda)$, for $i=1,2, \ldots, n$ where $\mathrm{W}_{i}^{(m)}$ and $\mathrm{W}_{i}^{(c)}$ are the weight associated with the mean and covariance matrices accordingly. Note: the parameter $\alpha_{A U K F}$ determines the spread of the sigma points around the mean $\overline{\mathbf{x}}, \beta_{A U K F}$ is used to incorporate prior knowledge of the distribution of the state $\hat{\mathbf{x}}$, and $\kappa$ is a secondary scaling parameter which is usually set to 0 . The fading factor in adaptive unscented Kalman filter is $\zeta=1 \times 10^{3}$. 
Particle Filter: The number of particles $N_{p}=100$, the resampling threshold for Effective Sample Size (ESS), $\beta_{P F}=0.5$, and the importance density was selected such that $q\left(\mathbf{x}_{k}^{(i)} \mid \mathbf{x}_{0: k-1}^{(i)}, \mathbf{y}_{1: k}\right)=p\left(\mathbf{x}_{k}^{(i)} \mid \mathbf{x}_{k-1}^{(i)}\right)$. To avoid the degeneracy of the particle filer, a resampling (selection) stage is introduced to multiply samples with high importance rations while eliminating samples with low importance. Several resampling schemes have been proposed in the literature including multinomial resampling, residual resampling, systematic resampling, etc. For details on resampling strategies please refer to [Arulampalam et al. 2002], [Douc et al. 2005], [Hol et al. 2007]. The resampling algorithm selected in this research is the multinomial resampling ${ }^{4}$. The multinomial resampling algorithm is presented in Table 3.1. The key idea behind multinomial resampling is to uniformly generate $N_{p}$ new independent particles from the old particle set. Each particle is replicated $N_{i}$ times i.e. each $\mathbf{x}^{(i)}$ produces $N_{i}$ children.

Table 3.1: Multinomial resampling algorithm [Chen 2003]

\section{Multinomial resampling algorithm}

1. (a) Produce a uniform distribution $u \sim \mathcal{U}(0,1)$.

(b) Construct a cumulative distribution function for the importance weights.

(c) Calculate $s_{i}=\sum_{j=1}^{i} \tilde{W}^{(j)}$.

2. Find $s_{i}$ such that $s_{i-1} \leq u<s_{i}$, the particle with index $i$ is chosen.

3. Given $\left\{\mathbf{x}^{(i)}, \tilde{W}^{(i)}\right\}$, for $j=1, \ldots, N_{p}$, generate new samples $\mathbf{x}^{(j)}$ by duplicating $\mathbf{x}^{(i)}$ according to the associate weight $\tilde{W}^{(i)}$.

4. Reset $W^{(i)}=1 / N_{p}$.

\footnotetext{
${ }^{4} \mathrm{~A}$ common problem with particle filter is weight degeneracy, which consist of unbounded increase in variance of the importance weight of the particles over time. The growth of variance is controlled by resampling methods which allows to maintain particle diversity.
} 


\subsection{Simulation Results and Discussion}

\subsubsection{Fault-free attitude estimation and control}

The purpose of this investigation is to compare the performance of adaptive unscented Kalman filter and particle filter under fault-free scenario. This scenario represents an optimal operating condition for any given system. Here, the control law, system dynamics and sensors are fully functioning and no external disturbances are present during simulation, i.e. $\vartheta_{k}=0$. Figure 3.1 shows the estimated angular velocity $\left(\hat{\omega}_{1}, \hat{\omega}_{2}, \hat{\omega}_{3}\right)$ using adaptive unscented Kalman filter and particle filter. Figure 3.2 shows the actual spacecraft attitude response $\left(q_{1}, q_{2}, q_{3}\right.$ and $\left.q_{4}\right)$, the observed attitude $\left(q_{1_{z}}, q_{2_{z}}, q_{3_{z}}\right.$ and $\left.q_{4_{z}}\right)$ and the estimated attitude response $\left(\hat{q}_{1}, \hat{q}_{2}, \hat{q}_{3}\right.$ and $\left.\hat{q}_{4}\right)$ using the adaptive unscented Kalman filter. Figure 3.3 shows the actual spacecraft attitude response $\left(q_{1}, q_{2}, q_{3}\right.$ and $\left.q_{4}\right)$ plotted against the observed attitude response $\left(q_{1_{z}}, q_{2_{z}}, q_{3_{z}}\right.$ and $\left.q_{4_{z}}\right)$ and the estimated attitude response $\left(\hat{q}_{1}, \hat{q}_{2}, \hat{q}_{3}\right.$ and $\left.\hat{q}_{4}\right)$ using the particle filter. Next, the estimated attitude response and angular velocities are evaluated based on error criteria.

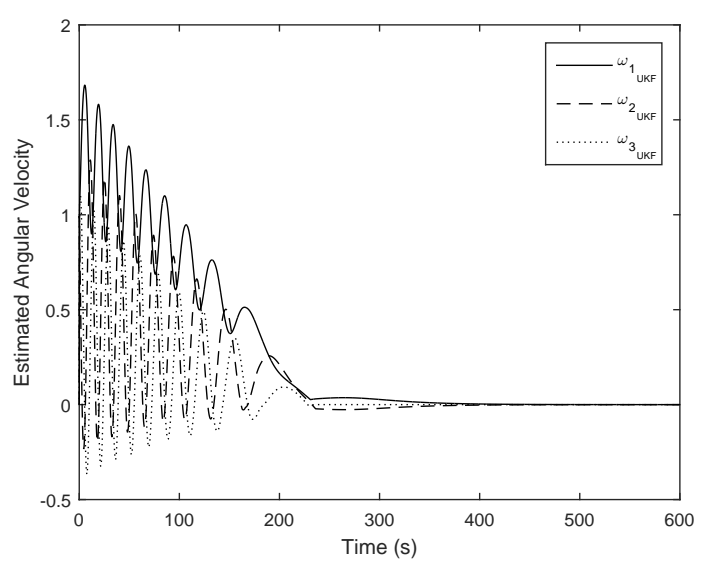

(a) Estimated angular velocity using AUKF

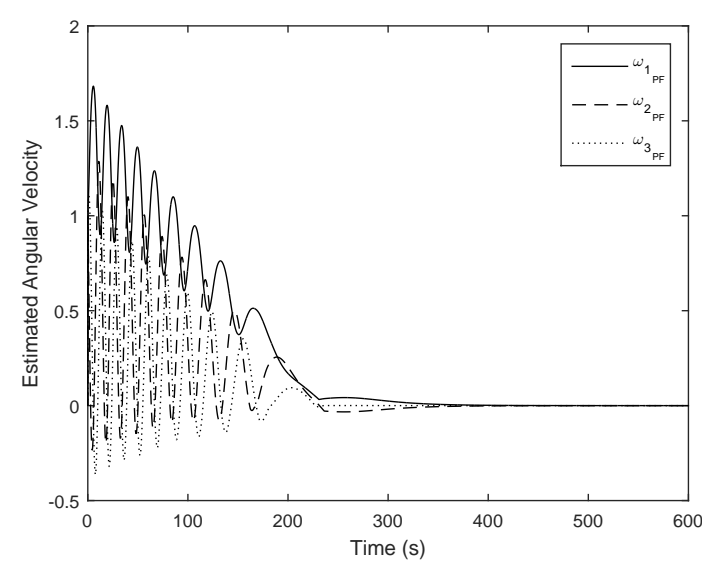

(b) Estimated angular velocity using PF

Figure 3.1: Estimated angular velocities. 


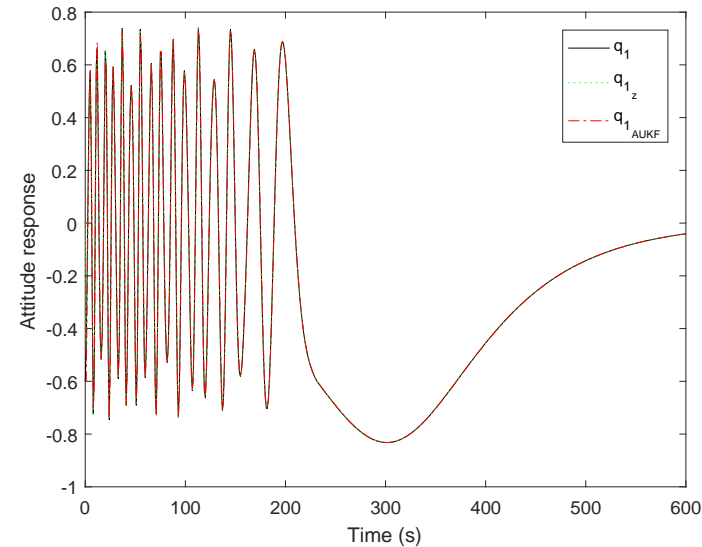

(a) Estimated attitude response: $\hat{q}_{1}$

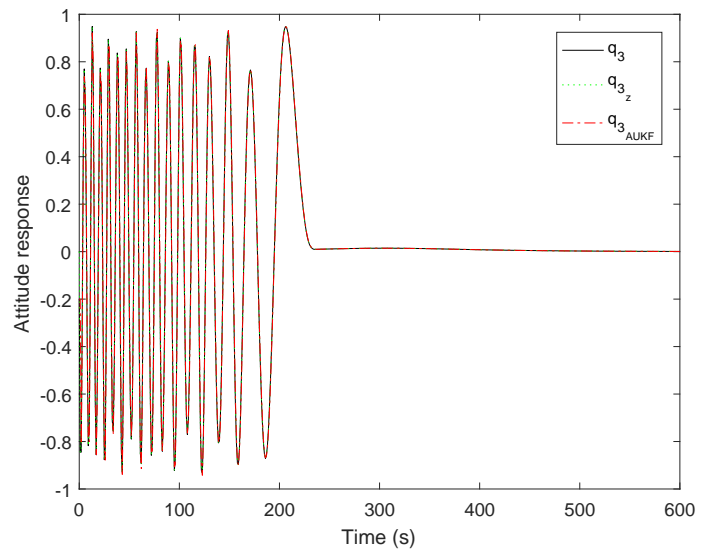

(c) Estimated attitude response: $\hat{q}_{3}$

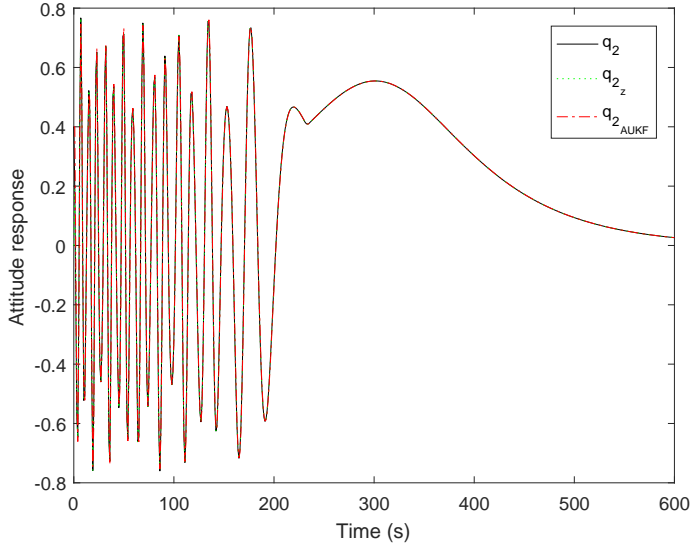

(b) Estimated attitude response: $\hat{q}_{2}$

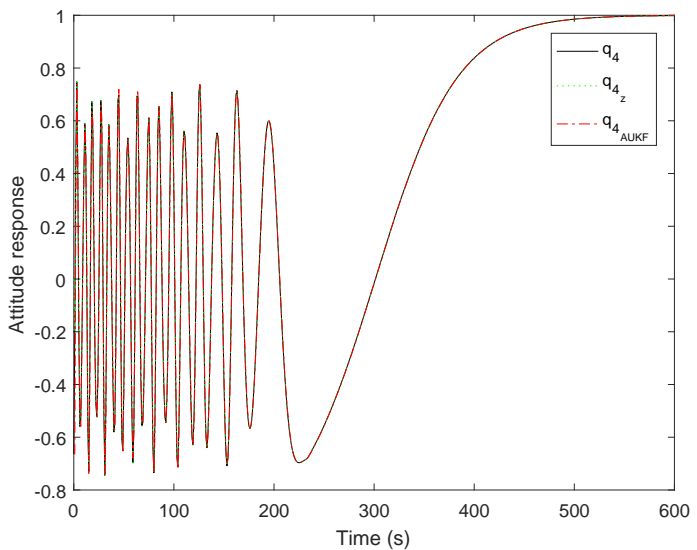

(d) Estimated attitude response: $\hat{q}_{4}$

Figure 3.2: Estimated attitude response using adaptive unscented Kalman filter. 


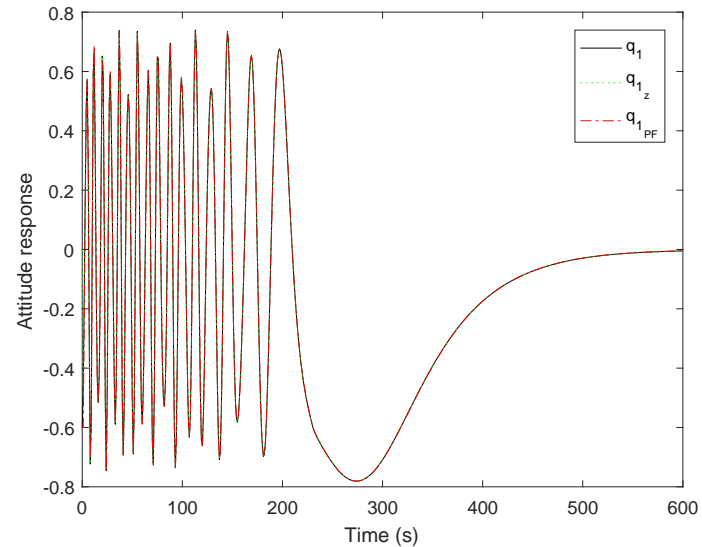

(a) Estimated attitude response: $\hat{q}_{1}$

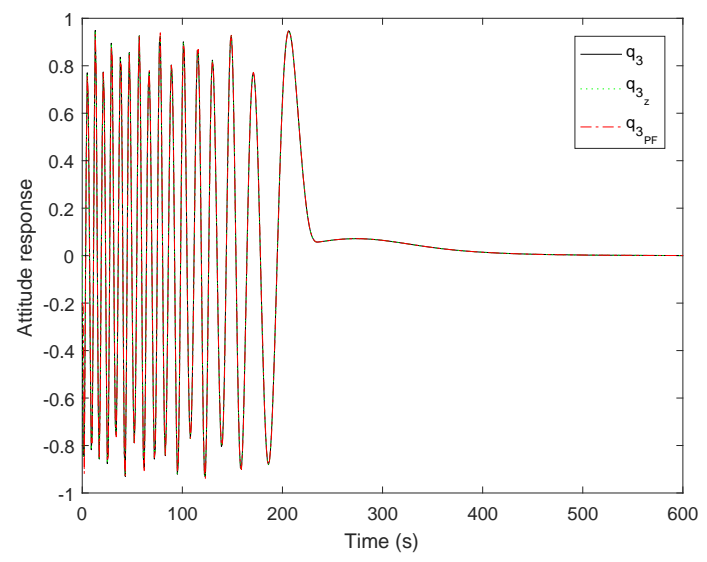

(c) Estimated attitude response: $\hat{q}_{3}$

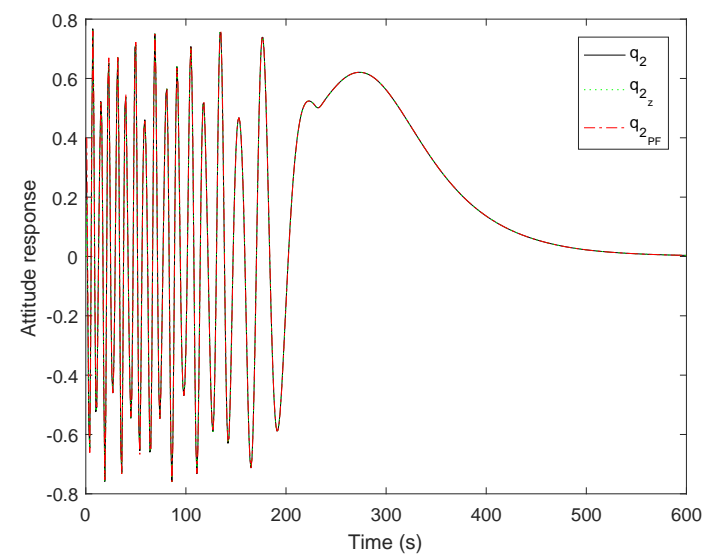

(b) Estimated attitude response: $\hat{q}_{2}$

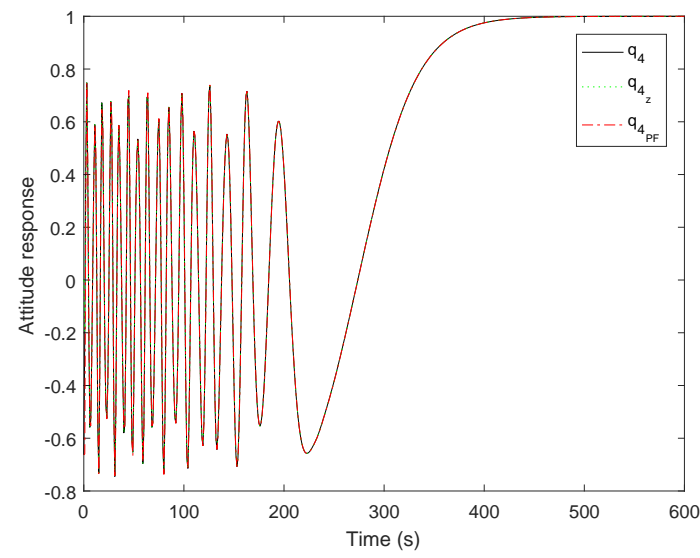

(d) Estimated attitude response: $\hat{q}_{4}$

Figure 3.3: Estimated attitude response using particle filter. 
Performance Evaluation: The attitude and angular velocities of a monolithic spacecraft under fault-free scenario were analyzed. The following discussion revolves around the errors generated between the state and estimated state in terms of quaternion error and angular velocity residuals. The errors are quantified with respect to the performance index root mean squared error (RMSE). The quaternion error between the attitude response and estimate attitude response is calculated according to Equation (3.5), and the angular velocity residual is calculated based on the difference between the state and estimated state, such that $\tilde{\omega}_{i}=\left(\omega_{i}-\hat{\omega}_{i}\right)$ for $i=1,2,3$. Figure 3.4 shows quaternion error and Figure 3.5 shows the residual between the angular velocity and estimated angular velocity. Considering the expense of computation time, the quaternion error and angular velocity residual shows that under the same control command, noise level, and external disturbances, the particle filter performs better than the adaptive unscented Kalman filter.

Figures 3.6 and 3.7 show the root mean squared error (RMSE) for the quaternion error and angular velocity residual. The figures show the initial estimation error decay as a function of time. It is apparent that root mean squared error is much smaller for particle filter estimation as compared to adaptive unscented Kalman filter estimation. The quaternion error initially ranges between 0.006 to 0.008 for particle filter estimation; however, quaternion error increases between 0.010 to 0.012 of initial error. The magnitude of angular velocity error is much smaller than quaternion error; however the same conclusion can be made. The estimation error of particle filter is much smaller than the estimation error of adaptive unscented Kalman filter.

Consider the time $t_{\varepsilon}$ as the time to enter the error region defined by $\varepsilon \in\left[\varepsilon_{\tilde{q}}, \varepsilon_{\tilde{w}}\right]$ where $\varepsilon_{\tilde{q}}= \pm 1 \times 10^{-3}$ and $\varepsilon_{\tilde{w}}= \pm 50 \times 10^{-6} \mathrm{rad} / \mathrm{sec}$. Table 3.2 shows the time required for the attitude response and angular velocity residual to converge within the defined error region; where $t_{\varepsilon \mathrm{AUKF}}$ and $t_{\varepsilon \mathrm{PF}}$ are the time it takes the AUKF and particle filter to converge bellow the error regions, and $\Delta t_{\varepsilon}=t_{\varepsilon \mathrm{AUKF}}-t_{\varepsilon \mathrm{PF}}$. The calculations shows the time when the system state vector enters the error region for particle filter which are 28-percent faster than adaptive unscented Kalman filter. Therefore, estimating the angular velocity using particle filter results in a faster convergence rate compared to adaptive unscented Kalman filter. 


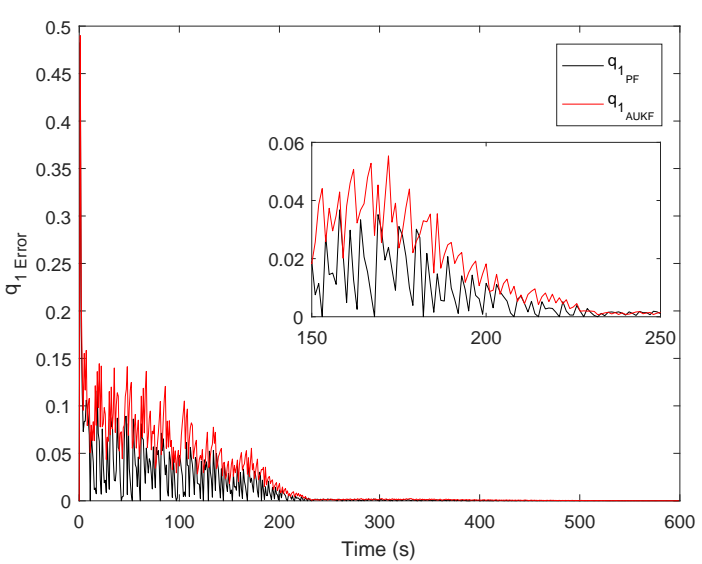

(a) Quaternion error $\left|\tilde{q}_{1}\right|$

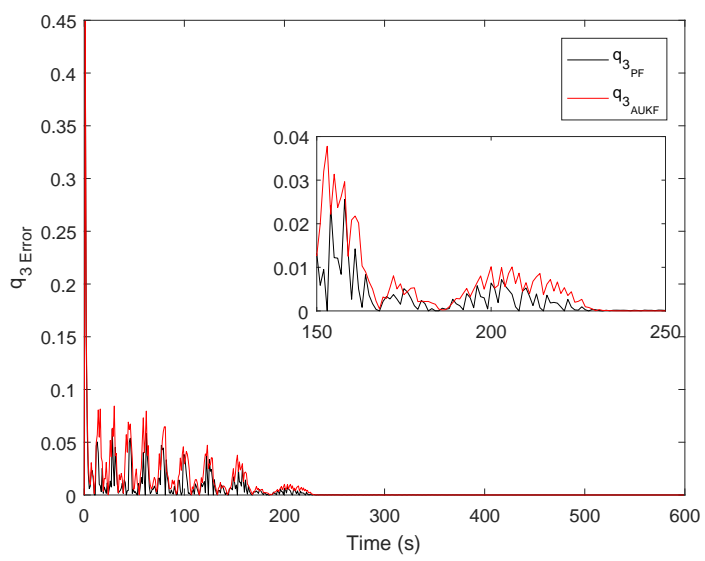

(c) Quaternion error $\left|\tilde{q}_{3}\right|$

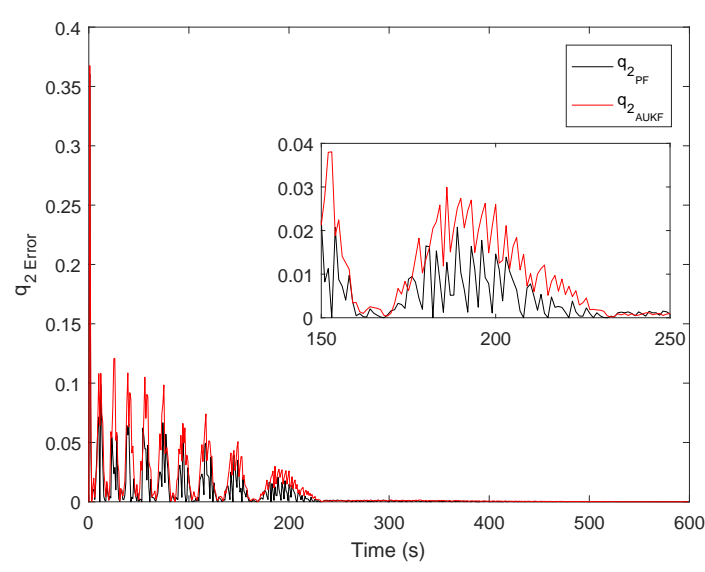

(b) Quaternion error $\left|\tilde{q}_{2}\right|$

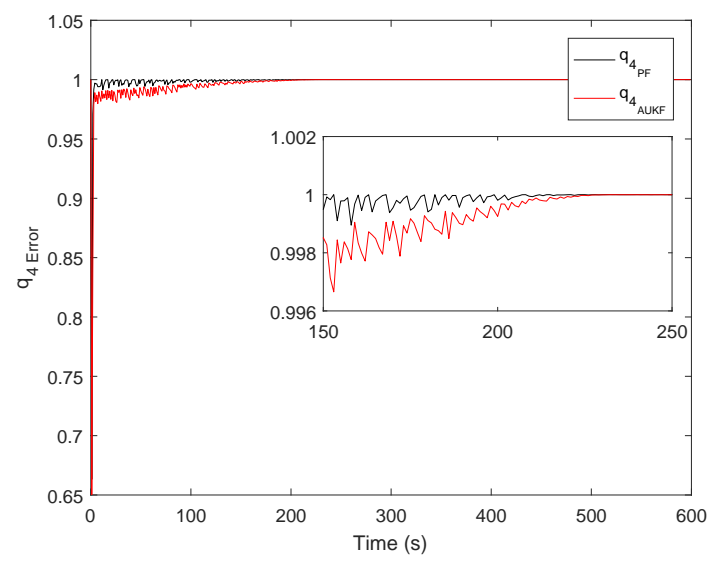

(d) Quaternion error $\left|\tilde{q}_{4}\right|$

Figure 3.4: Spacecraft attitude error. 


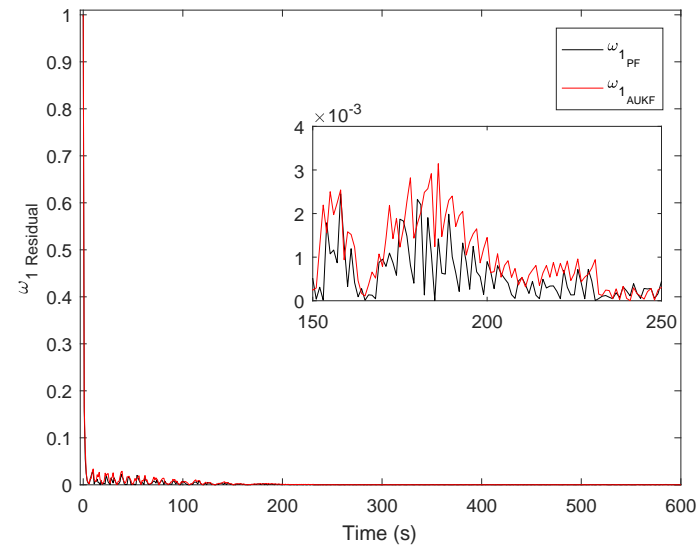

(a) Angular velocity residual: $\left|\tilde{\omega}_{1}\right|$

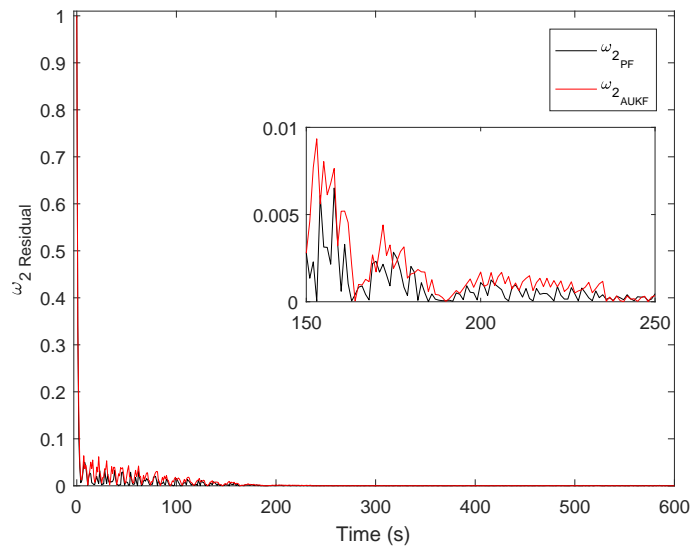

(b) Angular velocity residual: $\left|\tilde{\omega}_{2}\right|$

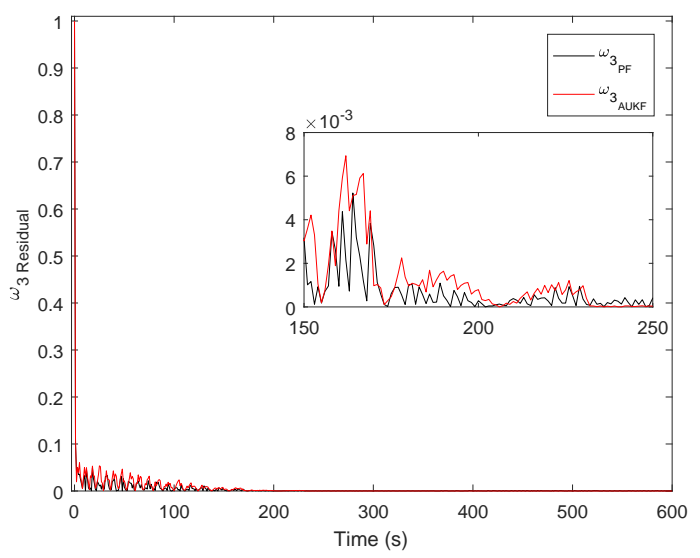

(c) Angular velocity residual: $\left|\tilde{\omega}_{3}\right|$

Figure 3.5: Angular velocity residual. 


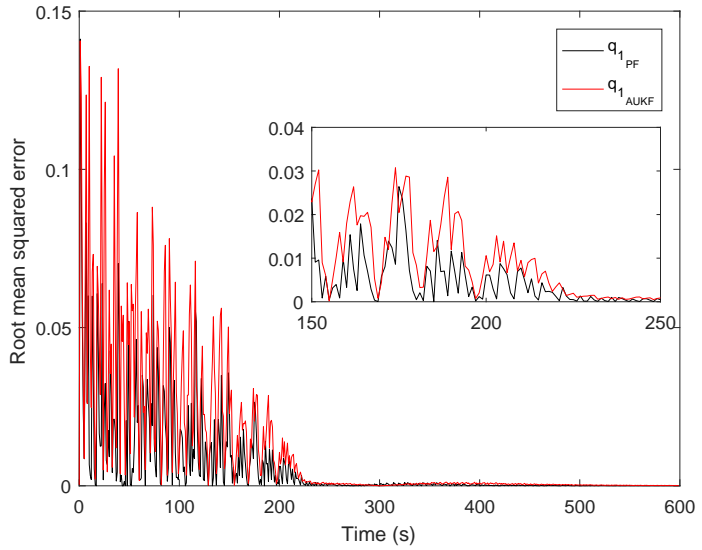

(a) RMSE: $q_{1}$

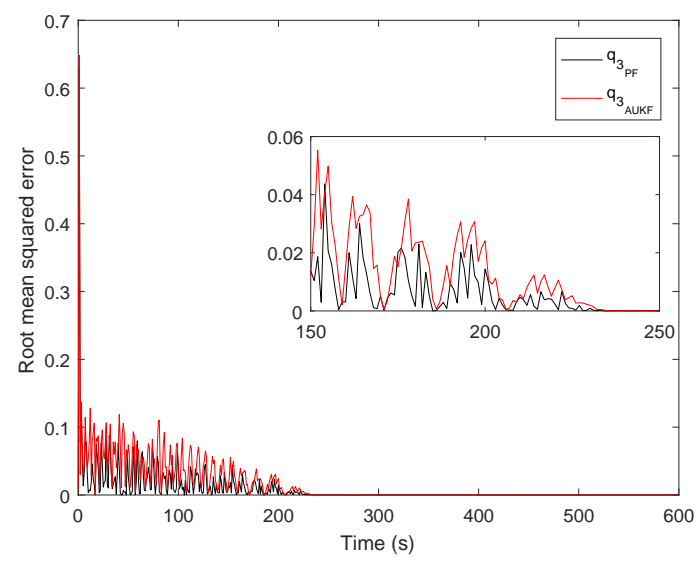

(c) RMSE: $q_{3}$

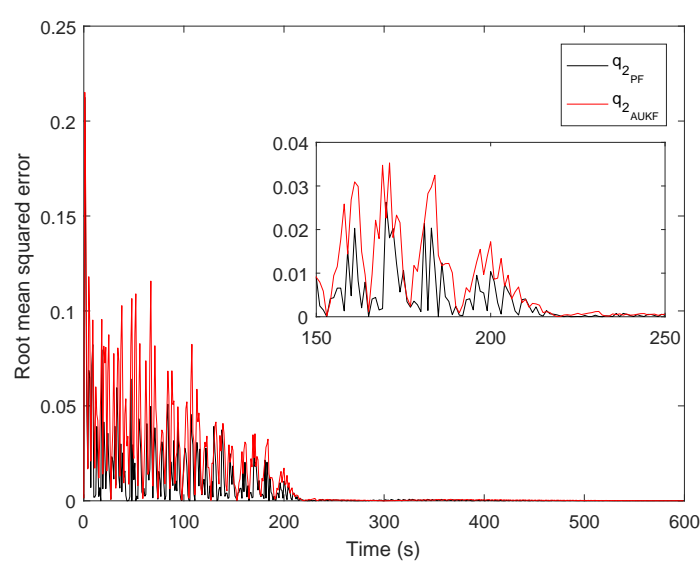

(b) RMSE: $q_{2}$

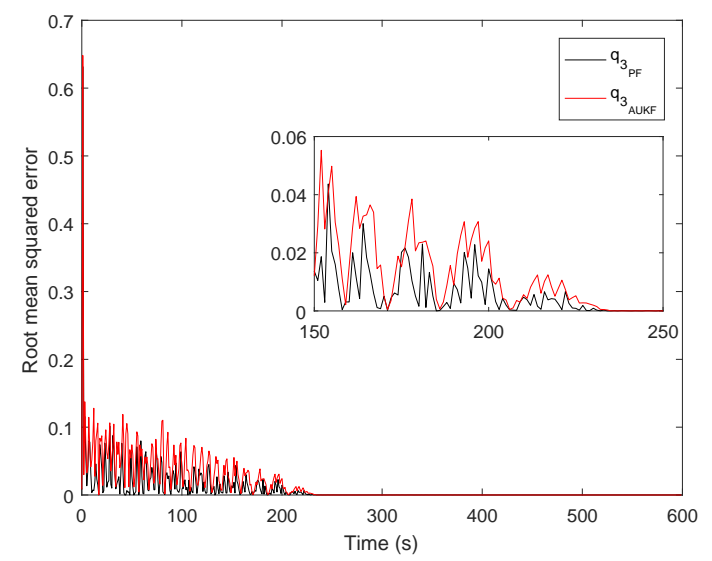

(d) RMSE: $q_{4}$

Figure 3.6: Root mean squared error for attitude response. 


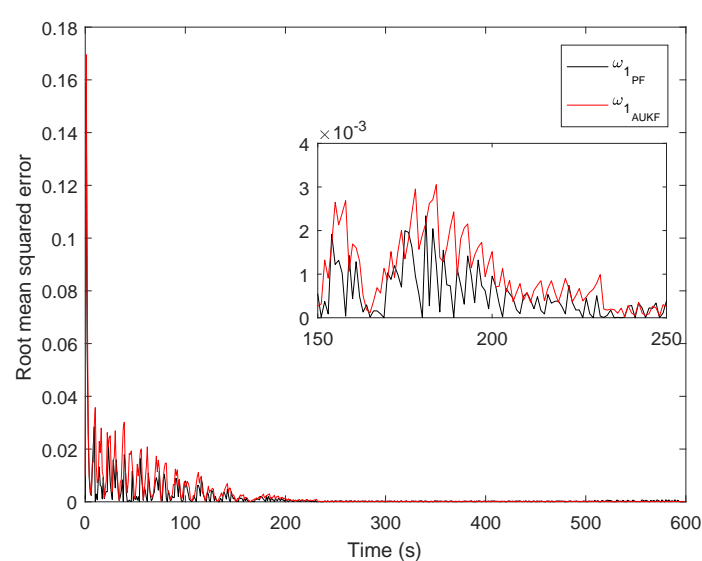

(a) RMSE: $\omega_{1}$

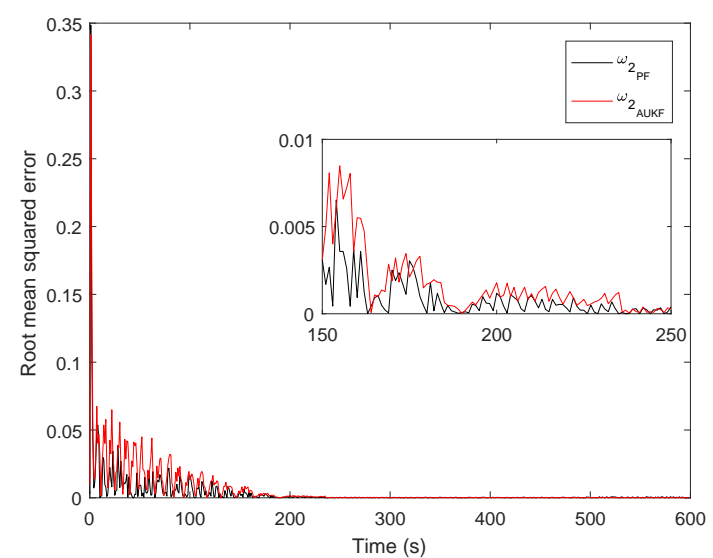

(b) RMSE: $\omega_{2}$

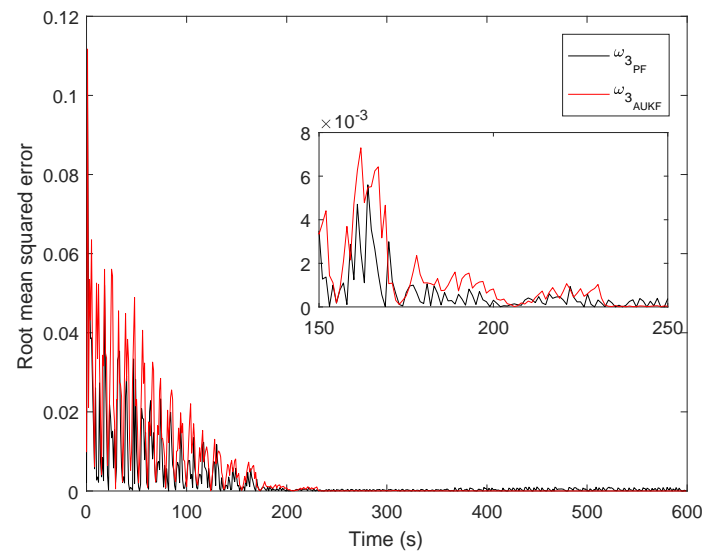

(c) RMSE: $\omega_{3}$

Figure 3.7: Root mean squared error for angular velocity. 


\subsection{Simulation Results and Discussion}

Table 3.2: Time required to enter error region

\begin{tabular}{|l|l|l|c|}
\hline \hline$t_{\varepsilon \mathrm{AUKF}}(\mathrm{sec})$ & $t_{\varepsilon \mathrm{PF}}(\mathrm{sec})$ & $\Delta t_{\varepsilon}(\mathrm{sec})$ & $\frac{t_{\varepsilon \mathrm{AUKF}}-t_{\varepsilon \mathrm{PF}}}{t_{\varepsilon \mathrm{AUKF}}} \times 100$ \\
\hline \hline 459.7 & 326.9 & 132.8 & $28.8 \%$ \\
\hline \hline
\end{tabular}

Based on the simulation results above, the PF provided better attitude estimations then AUKF since the samples of the AUKF are drawn in a specific and deterministic manner rather than at random as in the particle filter. The unscented transformation principle, the core of AUKF, is a deterministic sampling technique that chooses a set of specific sample points around the mean. Since the number of samples is small it may not represent adequately complicated posteriori distributions which may include biases i.e. skewness and kurtosis of distribution. The particle filter, however, relies on a probabilistic framework as a way to perform state estimation and deal with uncertainty over time. The continuous distributions of the particle filter are approximated by discrete random measures composed of weighted particles. The particles are samples of the unknown hidden states and the weights are the probability masses estimated using the Bayesian recursion. 
Chapter 3. Attitude Estimation and Control of Monolithic Spacecraft

\subsubsection{Uncertainty in moment-of-inertia and external disturbances}

Various fault and uncertainty cases are introduced with the purpose of varying the estimation sensitivity of AUKF and PF. The faults are introduced at the Spacecraft dynamics, control command, and sensor measurements. For simplicity, only residuals of attitude response and angular velocity along with performance indices are presented.

\section{Case I: Uncertainty in Moment of Inertia}

In the following case, abrupt faults introduces uncertainty in moment-of-inertia (MOI) in the spacecraft principal axes. The changes in MOI are compensated by the control command which is driven by the estimated states i.e. attitude response and angular velocity. The purpose is to estimate the attitude response and angular velocity of the spacecraft using the AUKF and PF filters and compare the estimation response of the filters. Two faults occur at the spacecraft during the simulation time. The first fault occurs 100-seconds after the start of simulation and second fault occurs 300-seconds and remains until the end of the simulation at 600 -seconds. Table 3.3 presents fault times and the corresponding MOI matrix. The MOI of the filter remains the same during the simulation.

Table 3.3: Uncertainty in moment-of-inertia.

\begin{tabular}{|c|c|}
\hline Time [sec] & Inertia matrix $\left[\mathrm{kg} \cdot \mathrm{m}^{2}\right]$ \\
\hline $0<t \leqslant 100$ & $J=\left[\begin{array}{lllllllll}20 & 1.2 & 0.9 ; & 1.2 & 17 & 1.4 ; & 0.9 & 1.4 & 15\end{array}\right]$ \\
\hline $100<t \leqslant 300$ & 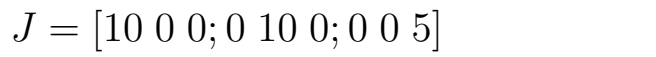 \\
\hline $300<t \leqslant 600$ & 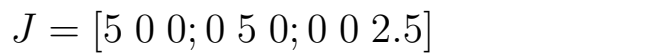 \\
\hline
\end{tabular}

Figure 3.8 shows the attitude response error $\tilde{q}_{i}$ for $i=1,2,3,4$. The attitude response expressed in terms of quaternion is calculated as $\tilde{q}_{i}=\hat{q}_{i}^{-1} \odot q_{i}$ according to equation (3.5). Figure 3.9 shows the angular velocity residual $\tilde{\omega}_{i}$ for $i=1,2,3$. The angular velocity residual is calculated as $\tilde{\omega}_{i}=\left(\omega_{i}-\hat{\omega}_{i}\right)$. Figure 3.10 shows the root mean squared error (RMSE) for the attitude response and Figure 3.11 shows the root mean squared error (RMSE) for the angular velocity. 


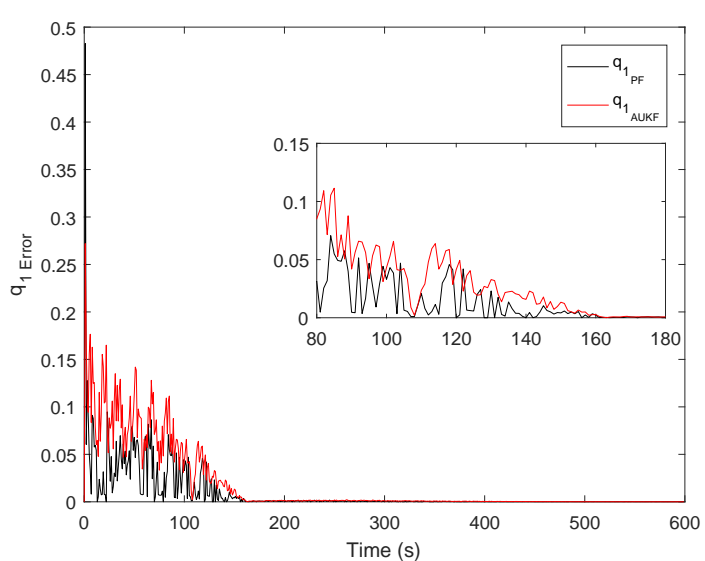

(a) Attitude response: $\left|\tilde{q}_{1}\right|$

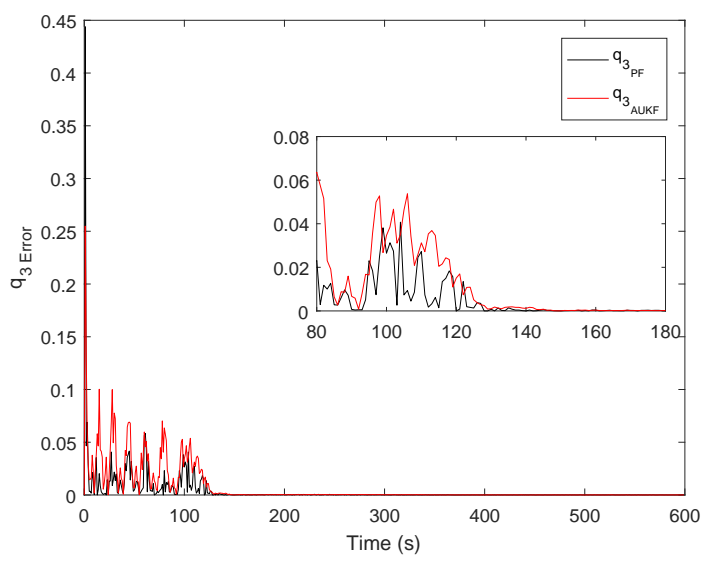

(c) Attitude response: $\left|\tilde{q}_{3}\right|$

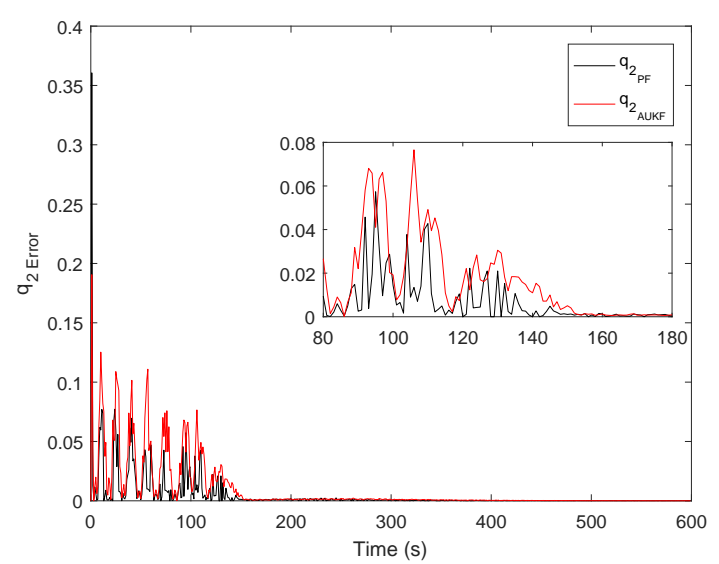

(b) Attitude response: $\left|\tilde{q}_{2}\right|$

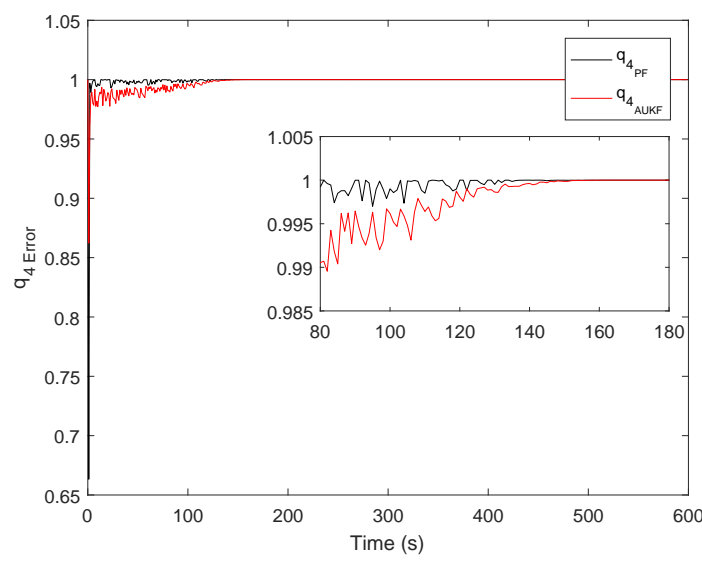

(d) Attitude response: $\left|\tilde{q}_{4}\right|$

Figure 3.8: Attitude response error under uncertainty in moment-of-inertia. 


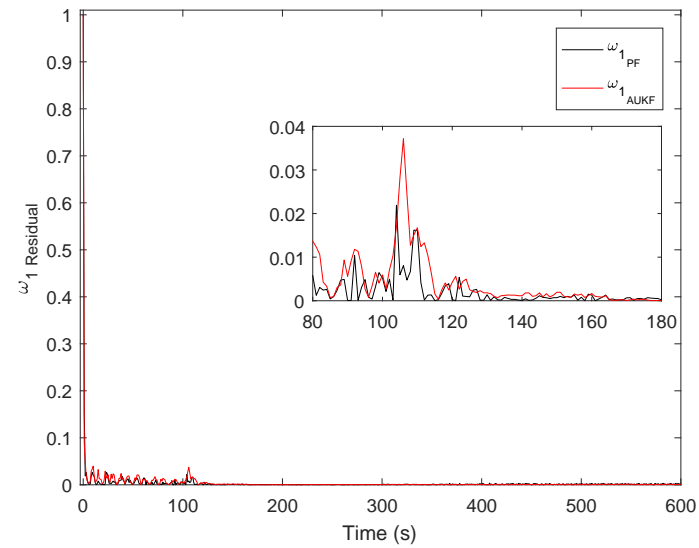

(a) Angular velocity residual: $\left|\tilde{w}_{1}\right|$

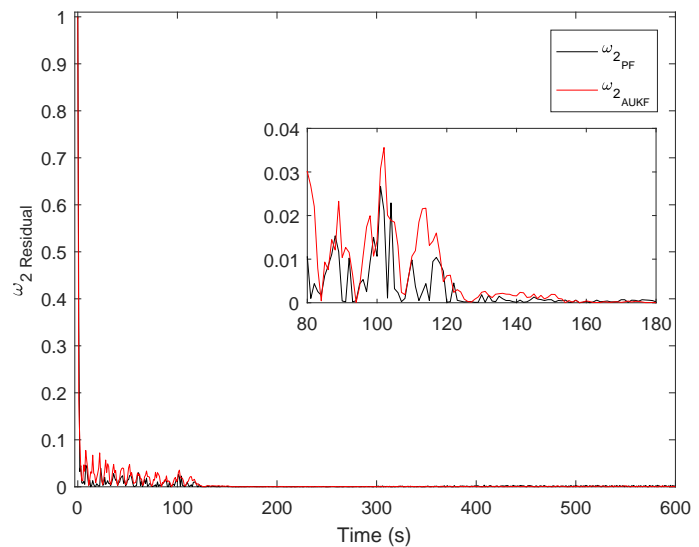

(b) Angular velocity residual: $\left|\tilde{w}_{2}\right|$

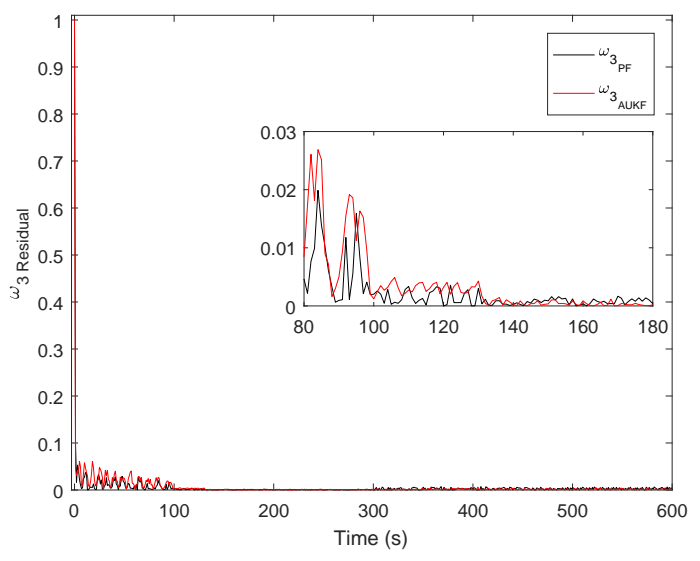

(c) Angular velocity residual: $\left|\tilde{w}_{3}\right|$

Figure 3.9: Angular velocity residual under uncertainty in moment-of-inertia. 


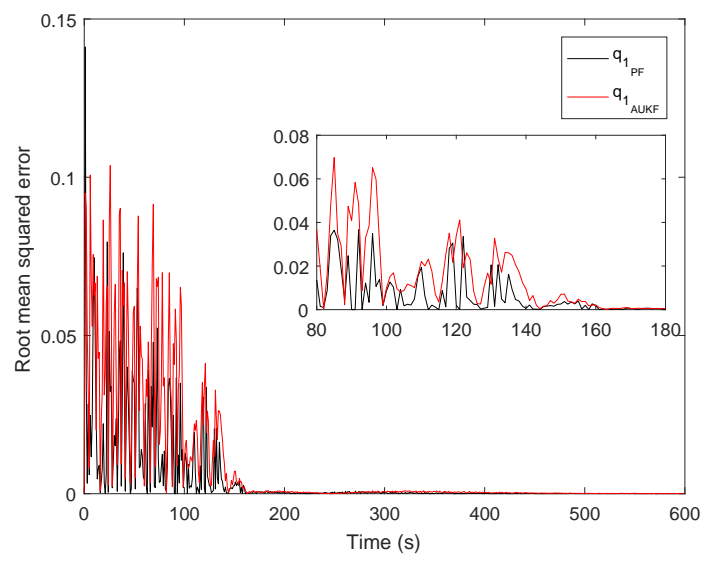

(a) RMSE: $\tilde{q}_{1}$

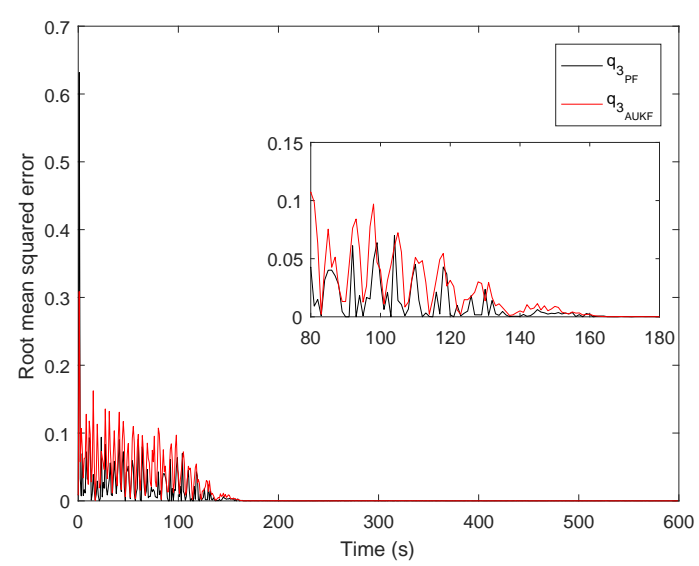

(c) RMSE: $\tilde{q}_{3}$

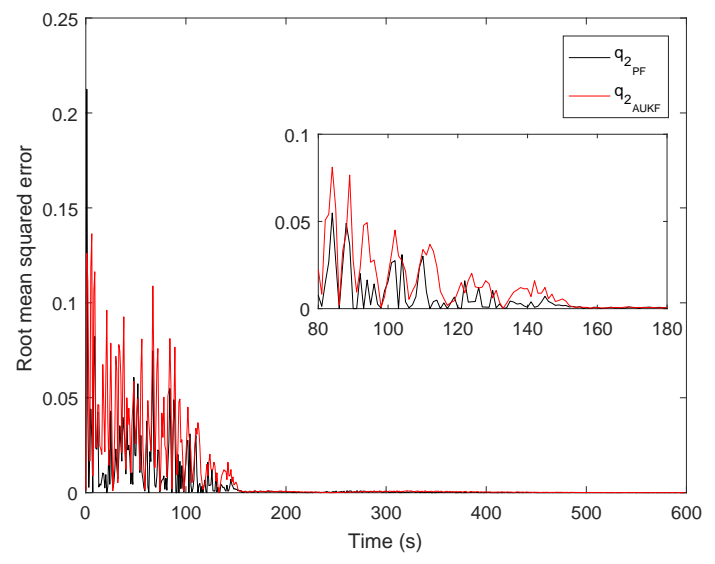

(b) RMSE: $\tilde{q}_{2}$

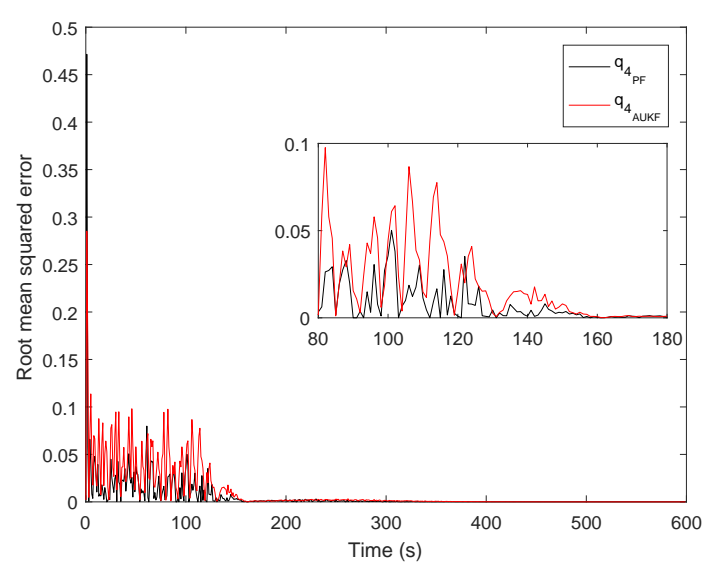

(d) RMSE: $\tilde{q}_{4}$

Figure 3.10: Root mean squared error of attitude response under uncertainty in momentof-inertia. 


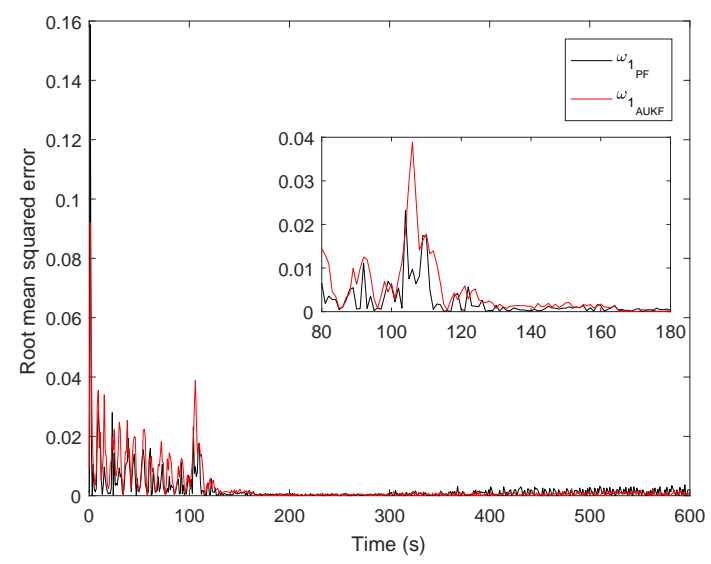

(a) RMSE: $\tilde{w}_{1}$

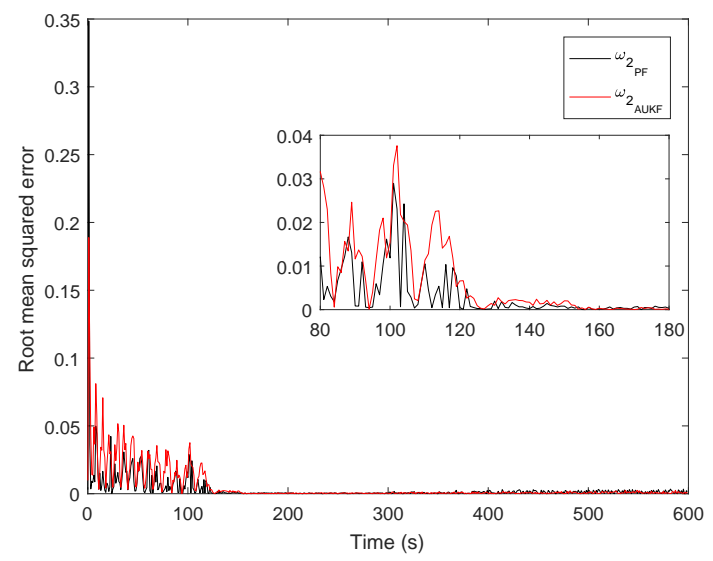

(b) RMSE: $\tilde{w}_{2}$

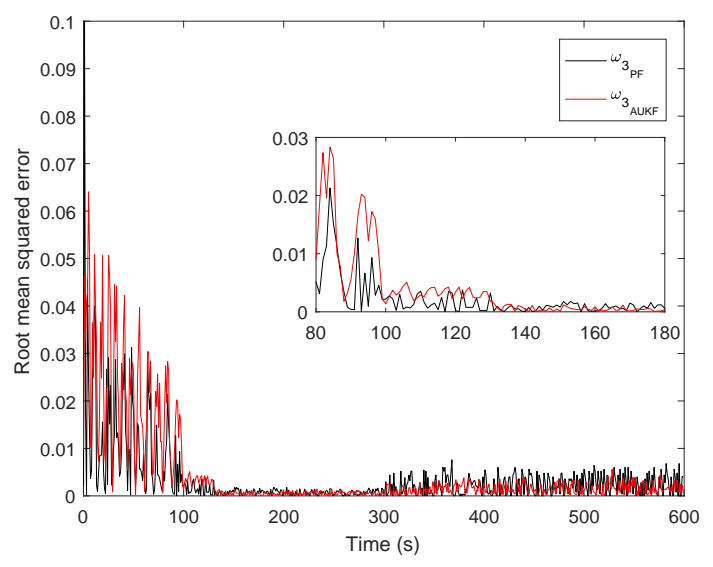

(c) RMSE: $\tilde{w}_{3}$

Figure 3.11: Root mean squared error of angular velocity under uncertainty in moment-ofinertia. 


\subsection{Simulation Results and Discussion}

\section{Case II: External disturbances}

In the following case, the spacecraft experiences an external disturbance and the fault occurred at the Spacecraft dynamics. Two external disturbances are introduced during the simulation time. At the start if the simulation there is no external disturbance present; therefore, $\vartheta=0$. The first disturbance occurs 100 -seconds after the beginning of simulation when an external force equivalent to $\vartheta=[\sin (t) ; \cos (t) ; \sin (2 t)]$ is applied for a duration of 350-seconds. The second disturbance occurs at $t=450$-second when a random external disturbance acts on each one of the principal axes of the spacecraft. Table 3.4 presents the fault time and the corresponding external disturbance.

Table 3.4: External disturbances.

\begin{tabular}{|l|l|l|}
\hline \hline Time $[\mathrm{sec}]$ & External disturbances \\
\hline \hline $0<t<=100$ & $\vartheta=d_{k}[\sin (t) ; \cos (t) ; \sin (2 t)] \quad$ where $\quad d_{k}=0$ \\
\hline $100<t<=450$ & $\vartheta=d_{k}[\sin (t) ; \cos (t) ; \sin (2 t)] \quad$ where $\quad d_{k}=1$ \\
\hline $450<t<=600$ & $\vartheta=d_{k} \times[$ Random; Random; Random $]$ where $d_{k}=0.1$ \\
\hline \hline
\end{tabular}

Figure 3.12 shows the attitude response error given the applied external disturbance. Figure 3.13 shows the angular velocity residual. The plots show that for a given external disturbance, the particle filter tend to estimate the spacecraft states faster then the adaptive unscented Kalman filter. In addition, the magnitude of error for the particle is smaller then the adaptive unscented Kalman filter. 


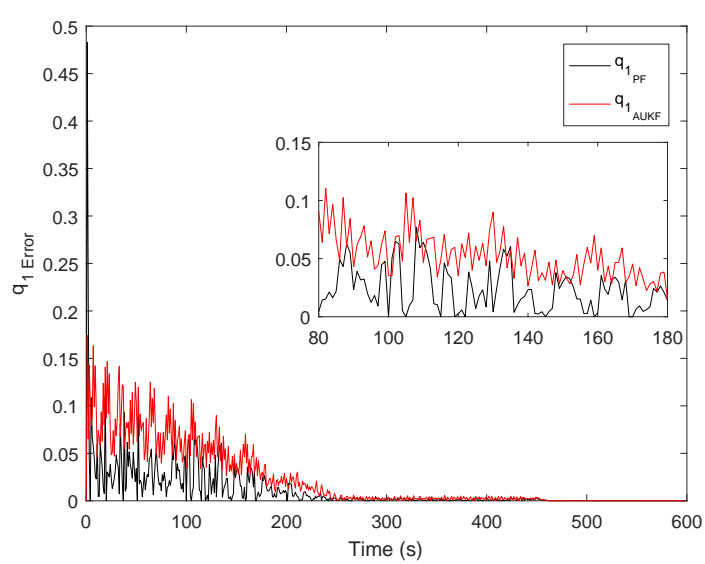

(a) Attitude response: $\left|\tilde{q}_{1}\right|$

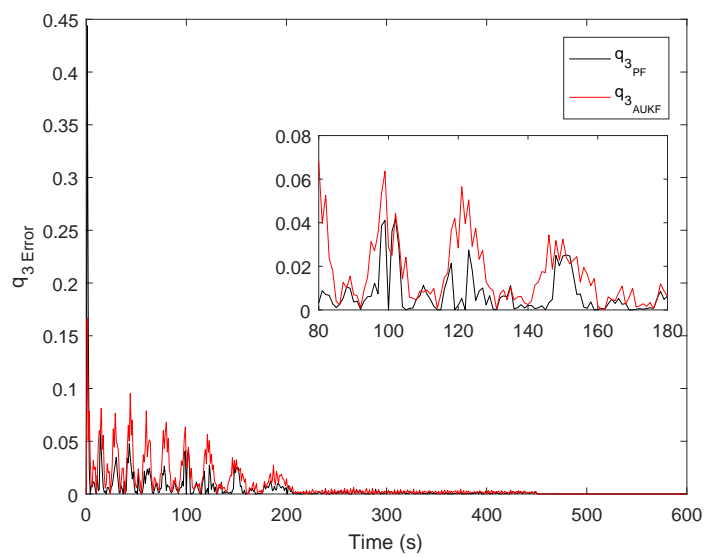

(c) Attitude response: $\left|\tilde{q}_{3}\right|$

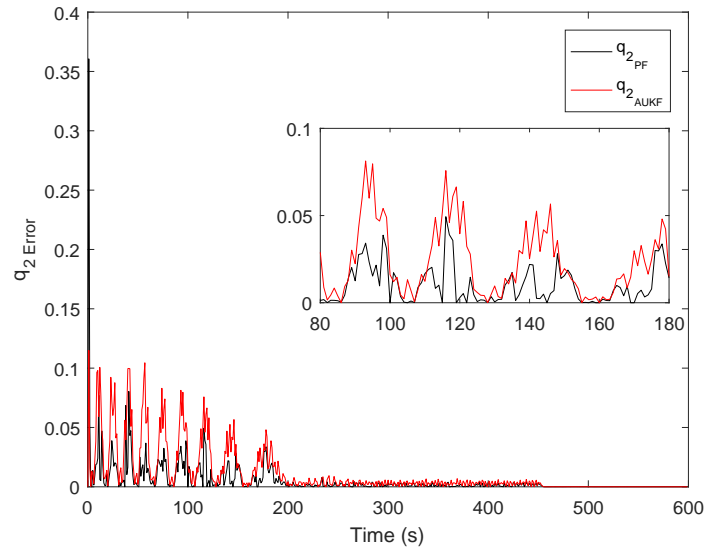

(b) Attitude response: $\left|\tilde{q}_{2}\right|$

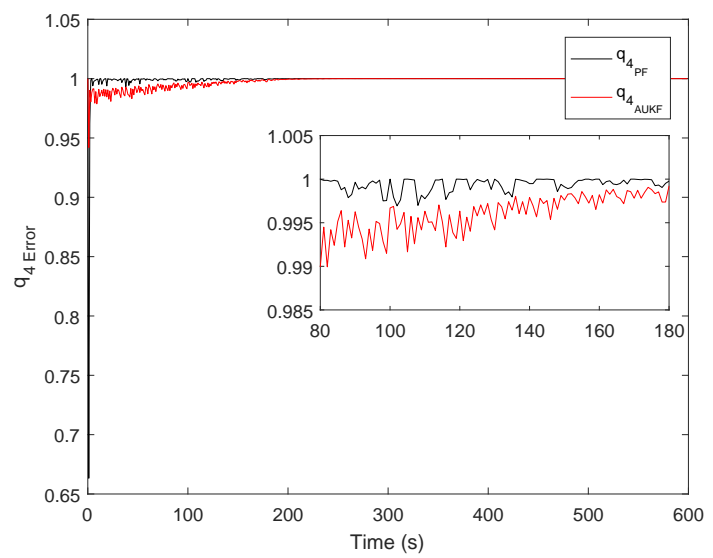

(d) Attitude response: $\left|\tilde{q}_{4}\right|$

Figure 3.12: Attitude response error under external disturbances. 


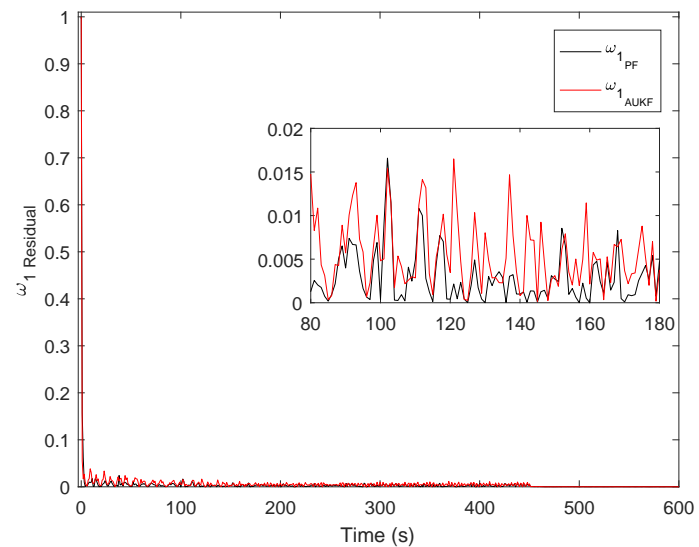

(a) Angular velocity residual: $\left|\tilde{\omega}_{1}\right|$

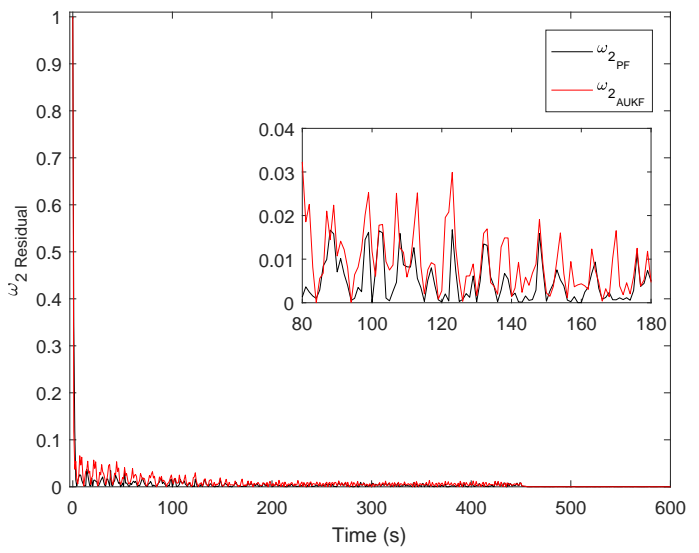

(b) Angular velocity residual: $\left|\tilde{\omega}_{2}\right|$

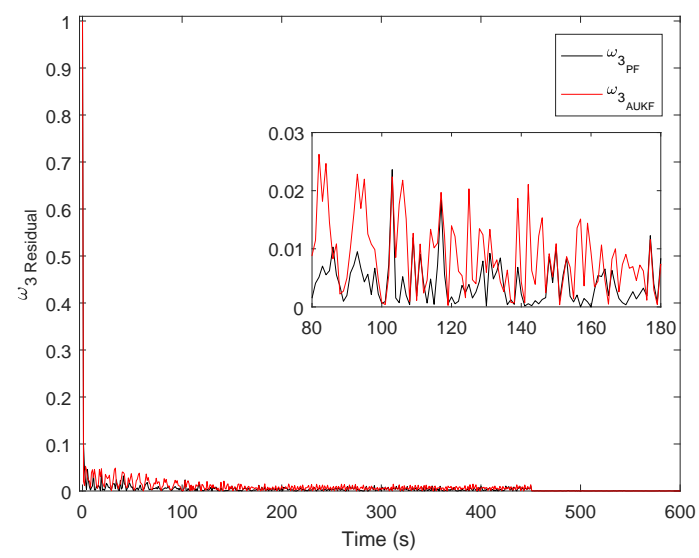

(c) Angular velocity residual: $\left|\tilde{\omega}_{3}\right|$

Figure 3.13: Angular velocity residual under external disturbances. 
Chapter 3. Attitude Estimation and Control of Monolithic Spacecraft

\subsubsection{Control command faults}

At this section, faults are introduced in the control command with the purpose of verifying the capacity of the filters to estimate the states. Two cases are investigated: (1) constant applied control commands are applied at different intervals during the simulations, and (2) no torque during stabilization.

\section{Case I: Constant applied control command}

In the following case, the spacecraft experience a fault where the control law sends out different torque magnitudes to each one of the principal axes. The first fault occurs 100 seconds after the beginning of simulation when the controller send out a constant control command with magnitude of $\tau_{1}=0.050, \tau_{2}=0.025$, and $\tau_{3}=0.075$. This fault last for 150 seconds, up until $t=250 \mathrm{sec}$, when the control command $\tau_{1}=0.050, \tau_{2}=0.025$ while $\tau_{3}$ is recovered and behaves as the desired control law (i.e., as per Equation (3.12)). This fault continuous for 100 seconds, up until $t=350 \mathrm{sec}$, when $\tau_{1}=0.050$, while $\tau_{1}$ and $\tau_{2}$ change as per desired control law. Finally, at $t=500 \mathrm{sec}$, the control command is fully recovered and send out a desired control law as per Equation (3.12). Table 3.5 presents the fault time and its corresponding applied control commands.

Table 3.5: Constant applied torque.

\begin{tabular}{|c|l|}
\hline \hline Time [sec] & Control command [Nm] \\
\hline \hline $0<t<=100$ & $\tau=J_{m}\left[-k_{2} \eta^{2 \nu-1}+J_{m}^{-1} \hat{\omega}^{\times} J_{m} \hat{\omega}-\theta^{2} \gamma_{2} \operatorname{sig}^{\alpha_{1}}(\overline{\tilde{q}})\right]$ \\
\hline $100<t<=250$ & $\tau_{1}=0.050, \tau_{2}=0.025, \tau_{3}=0.075$ \\
\hline $250<t<=350$ & $\tau_{1}=0.050, \tau_{2}=0.025, \tau_{3}=J_{m}\left[-k_{2} \eta^{2 \nu-1}+J_{m}^{-1} \hat{\omega}^{\times} J_{m} \hat{\omega}-\theta^{2} \gamma_{2} \operatorname{sig}^{\alpha_{1}}(\overline{\tilde{q}})\right]$ \\
\hline $350<t<=500$ & $\tau_{1}=0.050, \tau_{2}=\tau_{3}=J_{m}\left[-k_{2} \eta^{2 \nu-1}+J_{m}^{-1} \hat{\omega}^{\times} J_{m} \hat{\omega}-\theta^{2} \gamma_{2} \operatorname{sig}^{\alpha_{1}}(\overline{\tilde{q}})\right]$ \\
\hline $500<t<600$ & $\tau=J_{m}\left[-k_{2} \eta^{2 \nu-1}+J_{m}^{-1} \hat{\omega}^{\times} J_{m} \hat{\omega}-\theta^{2} \gamma_{2} \operatorname{sig}^{\alpha_{1}}(\overline{\tilde{q}})\right]$ \\
\hline \hline
\end{tabular}

Figure 3.14 shows the attitude response error and Figure 3.15 shows the angular velocity residual. 


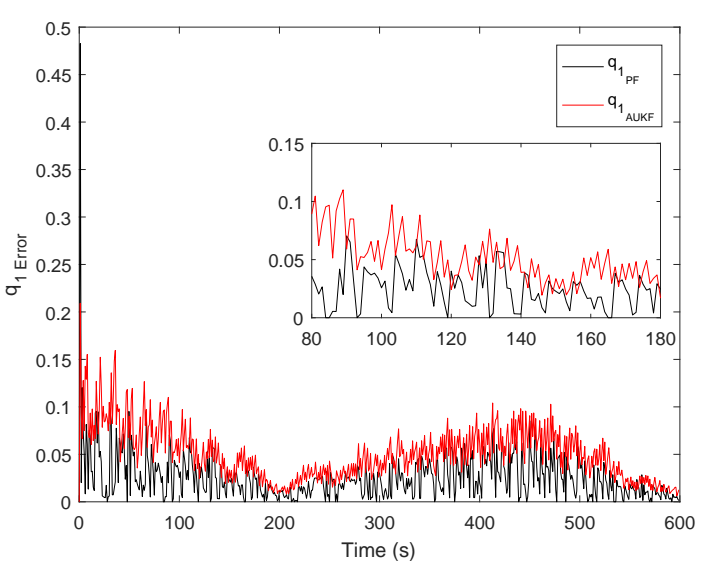

(a) Attitude response: $\left|\tilde{q}_{1}\right|$

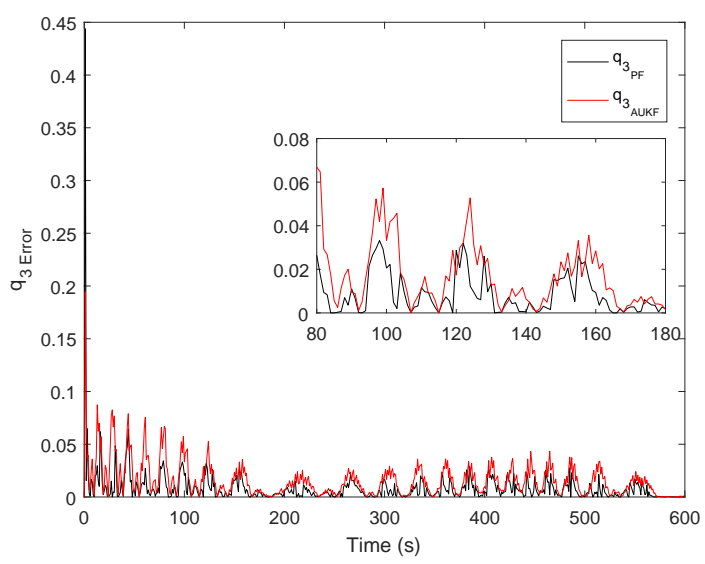

(c) Attitude response: $\left|\tilde{q}_{3}\right|$

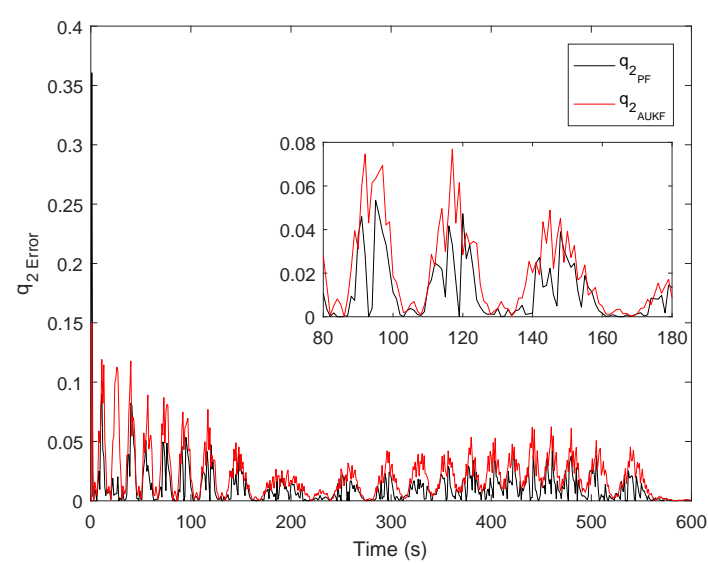

(b) Attitude response: $\left|\tilde{q}_{2}\right|$

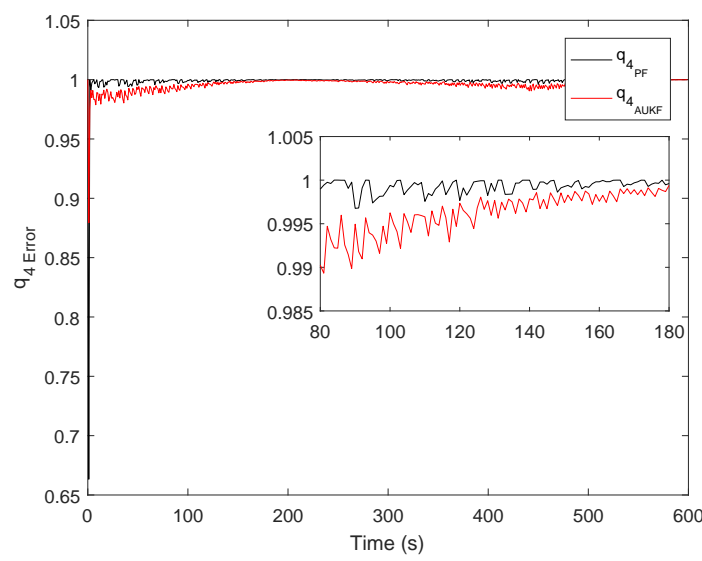

(d) Attitude response: $\left|\tilde{q}_{4}\right|$

Figure 3.14: Attitude response error under constant applied control commands. 


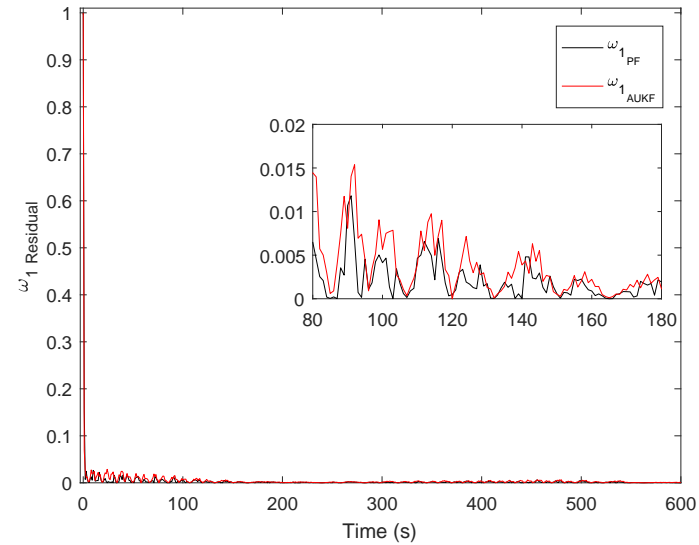

(a) Angular velocity residual: $\left|\tilde{\omega}_{1}\right|$

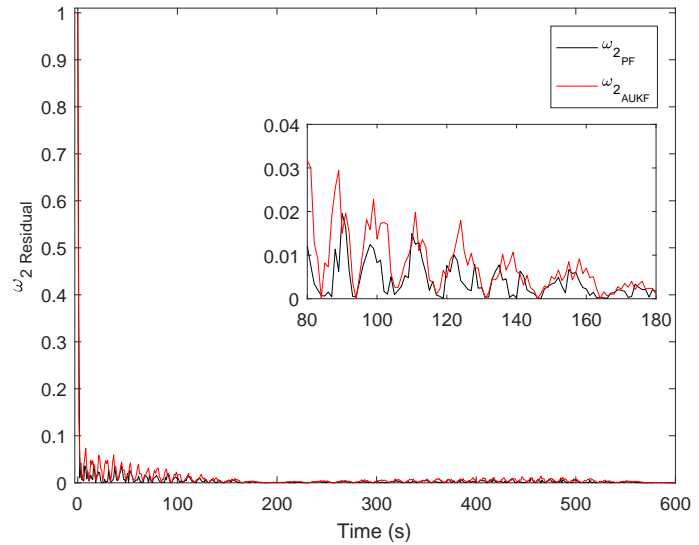

(b) Angular velocity residual: $\left|\tilde{\omega}_{2}\right|$

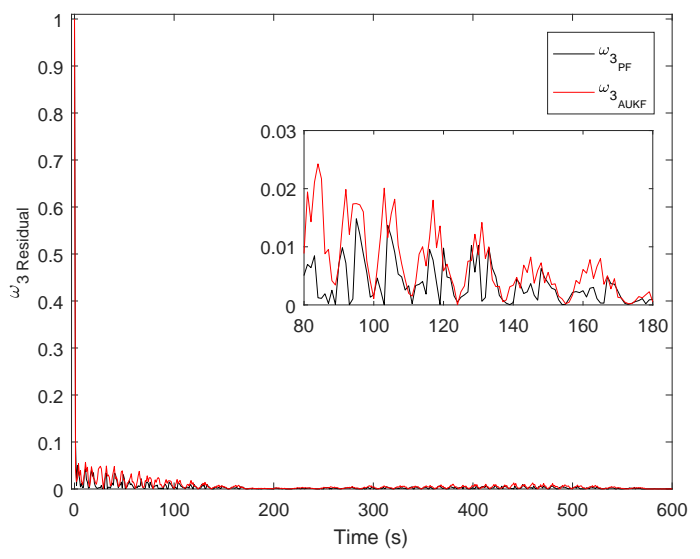

(c) Angular velocity residual: $\left|\tilde{\omega}_{3}\right|$

Figure 3.15: Angular velocity residual under constant applied control commands. 


\subsection{Simulation Results and Discussion}

\section{Case II: No control command during stabilization}

In the following case, the control command is failed at $t=50$ seconds and at $t=350$ seconds the control command is recovered. During the fault, the adaptive unscented Kalman filter is estimating the attitude response and the angular velocity of the spacecraft dynamics which experience an external disturbances of $\vartheta=0.1[\sin (t), \cos (t), \sin (2 t)]^{T}$. The initial conditions for the states are set as: $\bar{q}(0)=[-0.6,0.4,-0.2]^{T}, q_{4}(0)=\sqrt{1-\|\bar{q}(0)\|^{2}}$, and $\omega(0)=[0,0,0]^{T}$. Table 3.6 presents the fault time and its corresponding applied control torque.

Table 3.6: No control command during stabilization.

\begin{tabular}{|c|l|}
\hline \hline Time [sec] & Control command \\
\hline \hline $0<t<=50$ & $\tau=J_{m}\left[-k_{2} \eta^{2 \nu-1}+J_{m}^{-1} \hat{\omega}^{\times} J_{m} \hat{\omega}-\theta^{2} \gamma_{2} \operatorname{sig}^{\alpha_{1}}(\overline{\tilde{q}})\right]$ \\
\hline $50<t<=350$ & $\tau=0$ \\
\hline $350<t<600$ & $\tau=J_{m}\left[-k_{2} \eta^{2 \nu-1}+J_{m}^{-1} \hat{\omega}^{\times} J_{m} \hat{\omega}-\theta^{2} \gamma_{2} \operatorname{sig}^{\alpha_{1}}(\overline{\tilde{q}})\right]$ \\
\hline \hline
\end{tabular}

Figure 3.16 shows the attitude response error and Figure 3.17 shows the angular velocity residual.

\subsubsection{Sensor faults}

Sensors design requires a crucial consideration to satisfy a specific mission on a spacecraft. For given spacecraft applications, the sensor must provide an acceptable physical characteristics, meet performance specifications, and cope with interface sources and environmental conditions. The sensors are part of a larger system that is capable of processing and acting upon the information the sensor provide. This information is transmitted as a feedback signal to the rest of the system in a standardized manner. The purpose of this section is limited to estimation of attitude response and angular velocity of a spacecraft using the particle filter and adaptive unscented Kalman filter under the effect of sensor faults. 


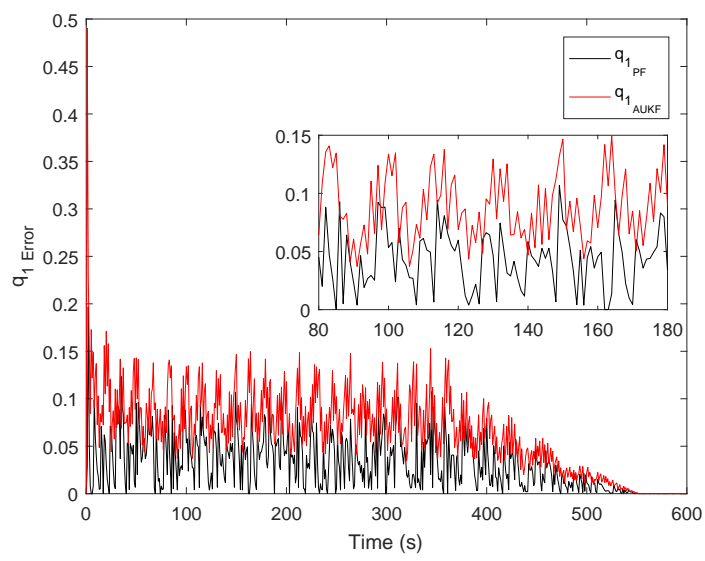

(a) Attitude response: $\left|\tilde{q}_{1}\right|$

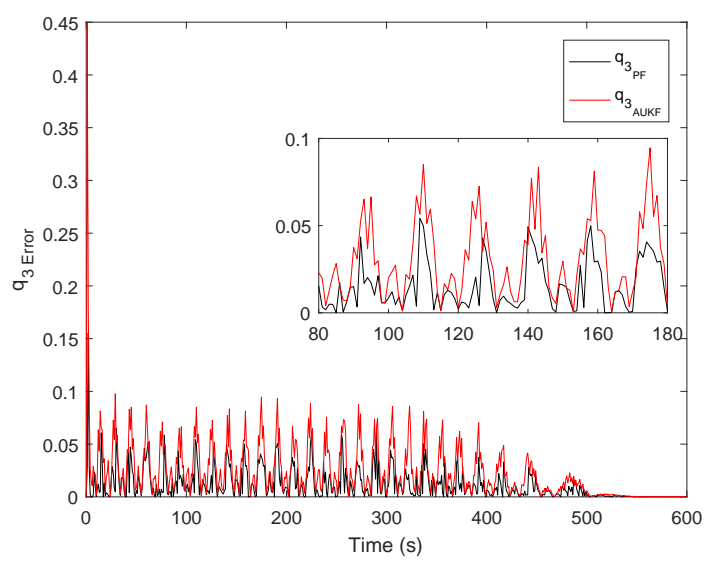

(c) Attitude response: $\left|\tilde{q}_{3}\right|$

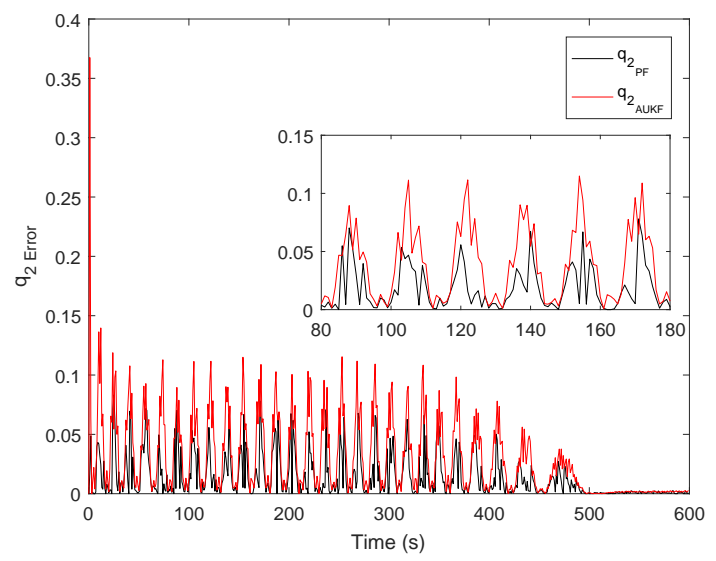

(b) Attitude response: $\left|\tilde{q}_{2}\right|$

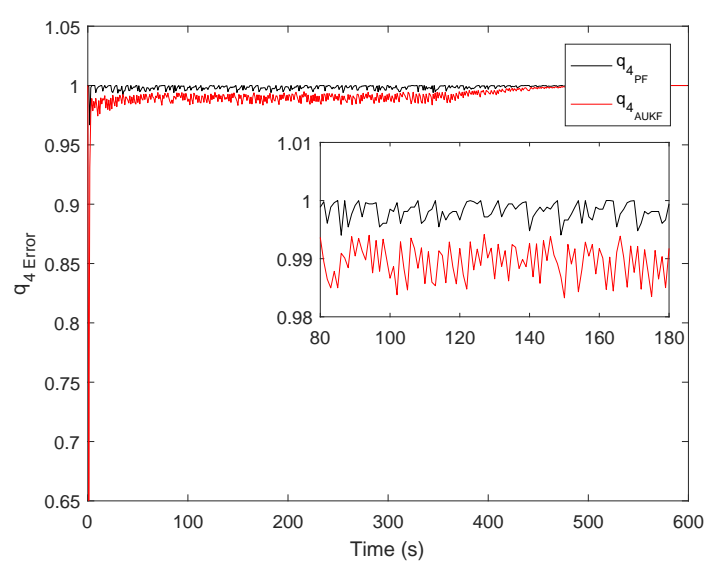

(d) Attitude response: $\left|\tilde{q}_{4}\right|$

Figure 3.16: Attitude response error when no control command is applied during stabilization. 


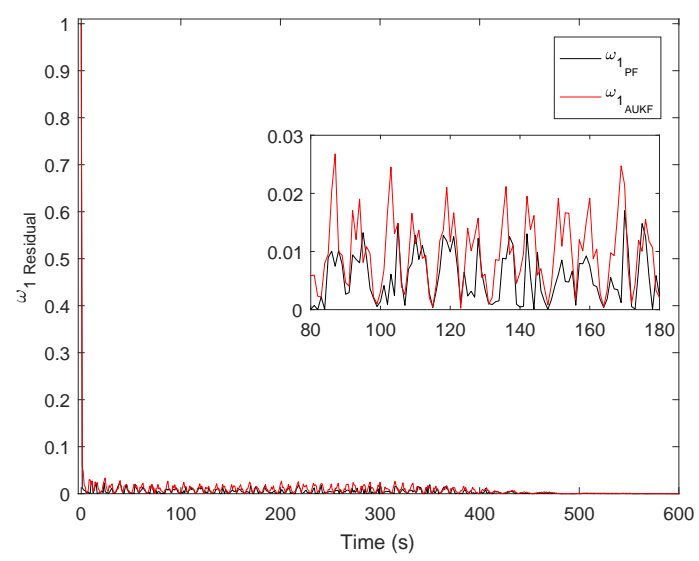

(a) Angular velocity residual: $\left|\tilde{\omega}_{1}\right|$

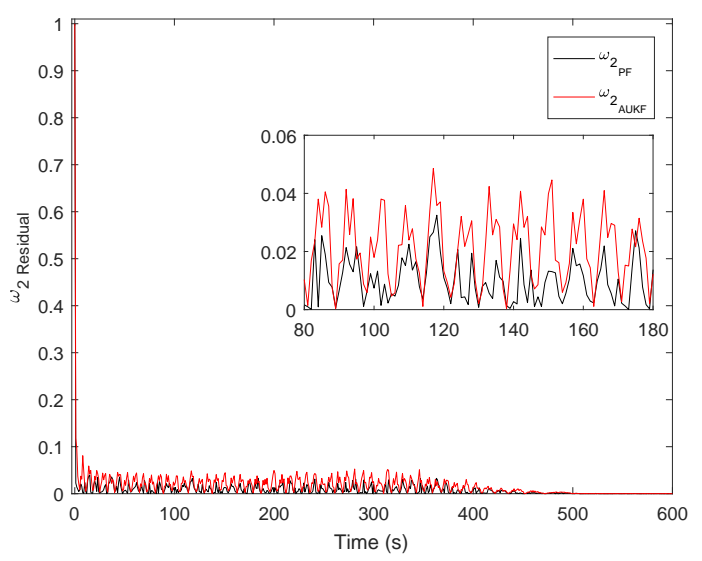

(b) Angular velocity residual: $\left|\tilde{\omega}_{2}\right|$

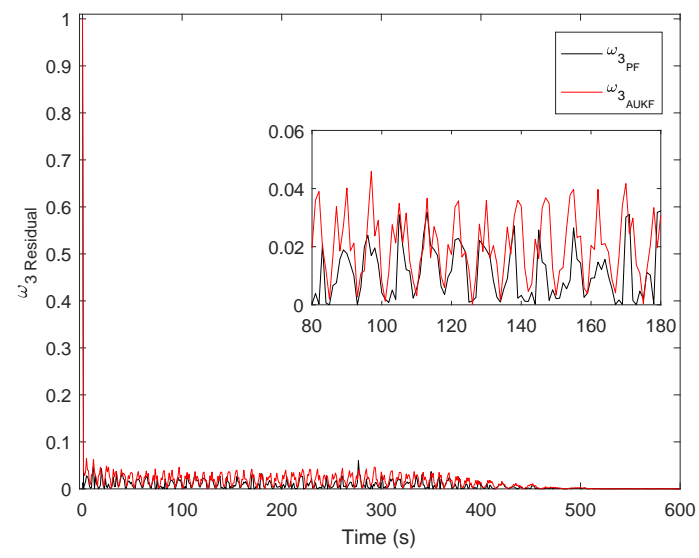

(c) Angular velocity residual: $\left|\tilde{\omega}_{3}\right|$

Figure 3.17: Angular velocity residual when no control command is applied during stabilization. 


\section{Case I: Heterogeneous measurements}

In the following scenario, the sensor experience various faults resulting in random measurement levels during the simulation. Various sensor measurements deteriorate the capacity to accurately estimate the attitude response and angular velocity of the spacecraft. For simulation purposes, the sensor sensitivity is altered multiple times to include random noise fluctuations with the scope of testing the capacity of PF and AUKF to estimate the spacecraft states. Initially, a normal operating conditions for sensor error is assumed in the state measurements; however, at $t=100$ seconds the noise in the measurements is changing at three intervals during the simulation. Table 3.7 presents the faults time and its corresponding noise sensitivity.

Table 3.7: Random noise levels.

\begin{tabular}{|c|lll|}
\hline \hline Time [sec] & \multicolumn{3}{|c|}{ Noise level } \\
\hline \hline $0<t<=100$ & Error $=0.05$, & $\mu=0$, & $\sigma=1 \times 10^{-3}$ \\
\hline $100<t<=300$ & Error $=2$, & $\mu=0$, & $\sigma=1 \times 10^{-6}$ \\
\hline $300<t<=500$ & Error $=0.001$, & $\mu=0$, & $\sigma=1 \times 10^{-5}$ \\
\hline $500<t<600$ & Error $=10$, & $\mu=0$, & $\sigma=1 \times 10^{-3}$ \\
\hline \hline
\end{tabular}

Figure 3.18 shows the attitude response error and Figure 3.19 shows the angular velocity residual of the spacecraft. The plots show that for a large measurement noise, the estimation error of the particle filter is smaller than the estimation error of the adaptive unscented Kalman filter; however, when the measurement noise level is small the estimation error of the adaptive unscented Kalman filter is smaller than the particle filter. This implies that, AUKF is capable of estimating the states better than PF for small measurement noise levels; whereas, the PF is capable of estimating the states better than AUKF for large noise measurements. The following conclusion is valid since the AUKF assign a predetermined constant weight to the distribution of the sigma points. When the measurement noise is small the constant weight assumption is valid and sufficient to estimate the state. However, when the measurement noise is large, the constant weight assumption is valid only to a certain extent, but not completely, since the sigma points have a larger covariance matrix 


\subsection{Simulation Results and Discussion}

resulting in a larger distribution of the sigma points. For a large measurement noise levels, the particle filter estimates the states more accurately than the AUKF for several reasons: First, the number of particles that estimates the distribution in PF is larger than the number of sigma points that estimates the distribution in the AUKF. Second, the weights assigned to the particles in the PF are assigned based on probability where a large weight implies higher estimate probability and a small weight imply less probability. The particles with less probability are vanished during the resembling stage while particles with large probabilities are replicated. The weight calculations in the PF differs from the AUKF since in the later the weights are constant throughout the simulation while in the PF the weight changes with probability. Third, a major assumption of the AUKF is that the sigma points are normally distributed whereas in the particle filter there is no assumption of particle distribution. The particle filter estimates the state distribution for any distribution even in the presence of kurtosis and skewness. 
Chapter 3. Attitude Estimation and Control of Monolithic Spacecraft

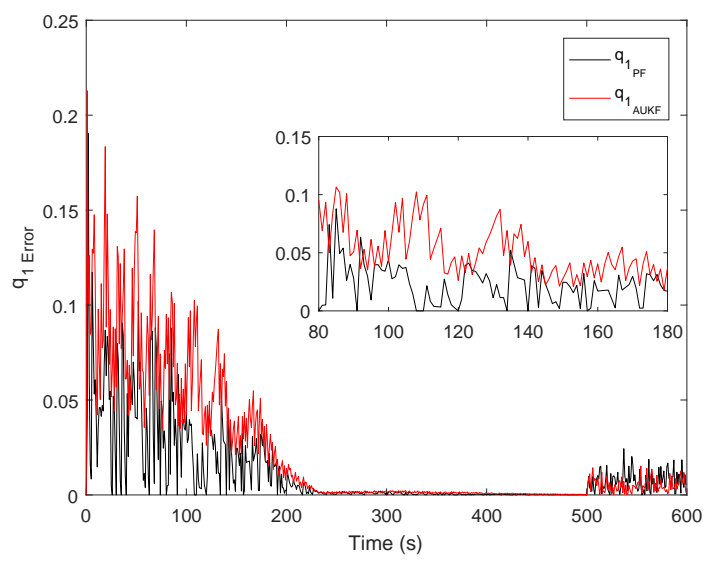

(a) Attitude response: $\left|\tilde{q}_{1}\right|$

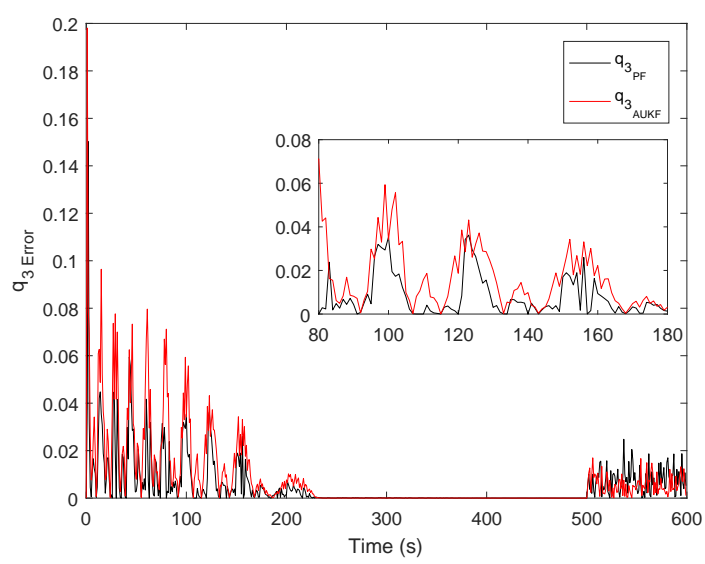

(c) Attitude response: $\left|\tilde{q}_{3}\right|$

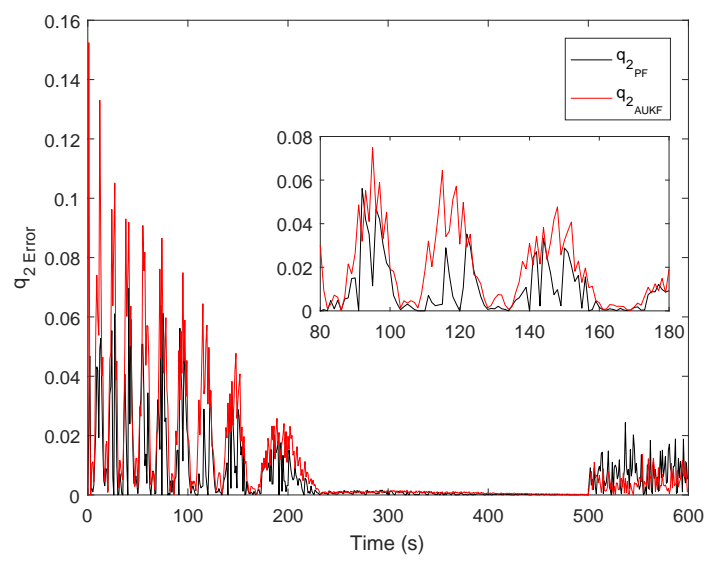

(b) Attitude response: $\left|\tilde{q}_{2}\right|$

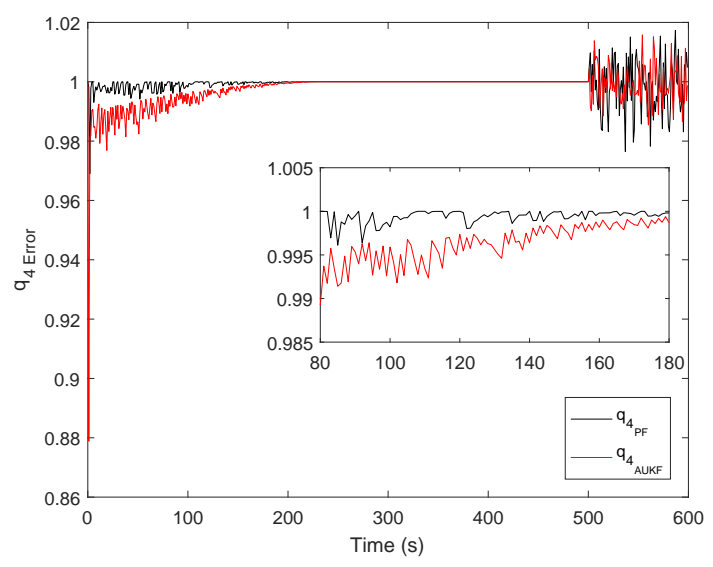

(d) Attitude response: $\left|\tilde{q}_{4}\right|$

Figure 3.18: Attitude response error under sensor fault. 


\subsection{Simulation Results and Discussion}

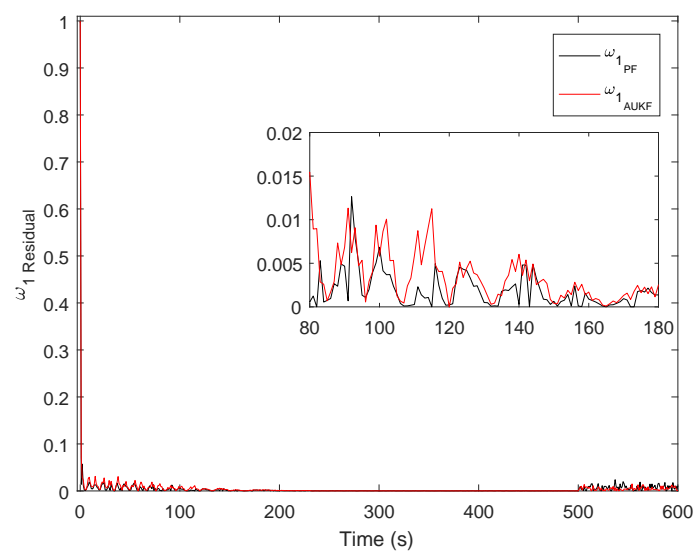

(a) Angular velocity residual: $\left|\tilde{\omega}_{1}\right|$

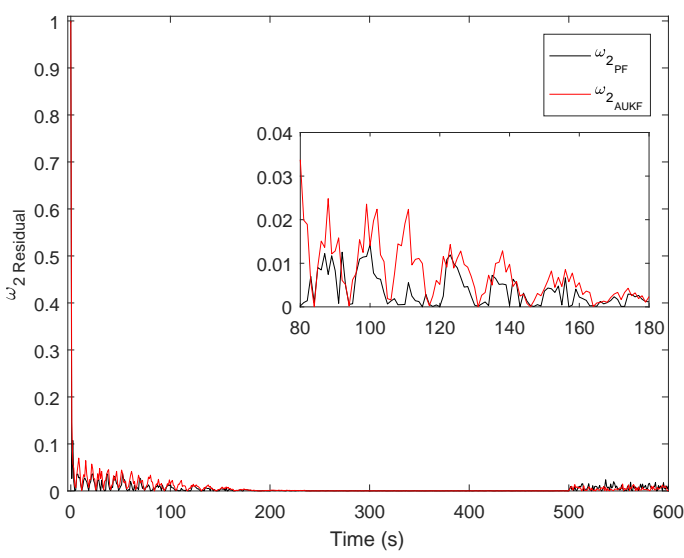

(b) Angular velocity residual: $\left|\tilde{\omega}_{2}\right|$

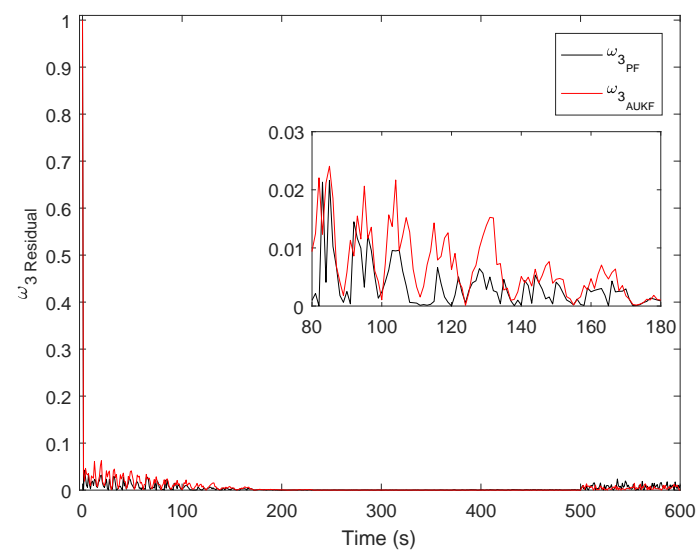

(c) Angular velocity residual: $\left|\tilde{\omega}_{3}\right|$

Figure 3.19: Angular velocity residual under sensor fault. 


\section{Chapter 3. Attitude Estimation and Control of Monolithic Spacecraft}

\subsection{Conclusions}

In this chapter, attitude estimation of monolithic spacecraft have be investigated in sequential setting under the presence of faults and unknown angular velocity measurements, where the attitude was represented by quaternion. The demand for online estimation and control requires a sequential update of attitude and angular velocity when a new measurements become available. Here, the attitude and the unknown angular velocity were estimated sequentially with adaptive unscented Kalman filter and particle filter. The successful design of the online particle filter for attitude estimation require to sample from a target distribution. Here, multinomial resampling algorithm was implemented for sequential state estimation. Since, the estimation process is done online, time-delay was introduced in the estimation algorithm to incorporate the estimation time between sequential measurements. Simulation results showed that PF provides better attitude estimations in comparison to AUKF since the samples of AUKF are drawn in a specific and deterministic manner rather than at random as in particle filter. The unscented transformation principle, the core of AUKF, is a deterministic sampling technique that chooses a set of specific sample points around the mean. Since the number of samples is small it may not represent adequately complicated posterior distributions which may include bias i.e. skewness, kurtosis or measurement outliers. The particle filter, however, relies on a probabilistic framework as a way to perform attitude estimation and deal with uncertainty over time. The posterior distribution of particle filter is approximated by discrete random measures composed of weighted particles. The particles are samples of the unknown hidden attitude and the weights are probability masses estimated using Bayesian recursions. In ordinary particle filtering, the larger the number of particles the more accurate the posterior distribution is. Therefore, the key requirement for particle filtering is to maintain sufficiently relatively large number of particles in order to adequately represent the posterior distribution. This number grows exponentially with the state vector dimensions. Low number of particles may cause degradation of the particles set and, consequently, might lead to divergence of the state estimate. In conclusion, considering the expense of computation time, the quaternion error under the same control input, noise level, external disturbance, etc. the particle filter estimated the attitude states better than adaptive unscented Kalman filter. 


\section{CHAPTER 4}

\section{Distributed Attitude Estimation and Control of Spacecraft Formation Flying}

In this chapter, an efficient and accurate distributed nonlinear filtering techniques are proposed to estimate the unmeasurable angular velocity and reference angular acceleration of Spacecraft Formation Flying (SFF). The spacecraft formation is comprised of $N$ spacecraft and a virtual leader which exchange partial information with other spacecraft in the formation. Each spacecraft runs its own filter to accurately approximate the posterior distribution of the unmeasurable angular velocity while a distributed filter is incorporated to obtain a reference angular acceleration estimates of the virtual leader. The attitude is represented by modified Rodrigues parameters (MRP). Two nonlinear filtering techniques are investigated herein, the adaptive unscented Kalman filter (AUKF) and particle filter $(\mathrm{PF})$. The AUKF and PF in parallel with a distributed filter and an asymptotic output feedback control law are utilized to simultaneously track a common time-varying reference attitude while the reference attitude is available only to a subset of the spacecraft in the formation. The aim is to motivate the application of particle filtering technique for distributed estimation of spacecraft formation flying. Simulation results show state estimation in spacecraft formation tracking in fault-free and fault-prone scenarios under presence of faults and uncertainty at the spacecraft dynamic models, control laws, and measurement models. The faults are unknown the filters and the control laws. The performance comparison between AUKF and PF is illustrated with numerical simulation through calculations of formation-keeping error and station-keeping error. 


\section{Chapter 4. Distributed Attitude Estimation and Control of Spacecraft} Formation Flying

\subsection{Introduction}

Spacecraft Formation Flying (SFF) is an active research topic that deals with maintaining the state of formation between spacecraft. A growing number of space missions have been identified as suitable to utilize SFF technology; where, the idea is to distribute the functionality of large spacecraft among smaller, less expensive cooperative spacecraft. Estimating the state of formation becomes a serious challenge in the presence of system faults and external disturbances ${ }^{1}$ which causes a drift in both position and formation center of the spacecraft. The issue with attitude estimation of spacecraft in formation flying gave rise to a great level of interest in both scientific and defense communities to develop systems for precise state estimation of spacecraft formation flying and autonomous rendezvous.

Modern guidance and navigation systems today rely on some form of nonlinear filtering algorithm based on probabilistic inference to compute the state estimate of the spacecraft. The nonlinear filter estimates the system dynamics by filtering out noisy measurements based on a two step iterative process of prediction and correction. The errors in the dynamic process and measurement model are assumed to have a deterministic model and covariance model (i.e., zero-mean Gaussian process) and some form of an optimality conditions which minimizes the error covariance between estimated state and system state.

In the scientific literature, SFF and information sharing among spacecraft take on several different formation estimation architectures (FEA). These include centralized, distributed, and decentralized architectures [Kang et al. 2001]:

1. Centralized estimation architecture include a single formation-wide master filter that collect sensor measurements, control inputs, and spacecraft configuration in order to estimate the formation states. This is the simplest estimation architecture where all the necessary estimations occurs at a common processing station. Resource allocation is easy because the master filter is fully aware of the activities of each spacecraft and has an overall view of the entire formation. Centralized estimation architecture has

\footnotetext{
${ }^{1}$ External disturbance may result from gravitational perturbation, atmospheric drag, solar radiation pressure, electromagnetic forces, and/or Earth oblateness (differential $J_{2}$ ).
} 


\subsection{Introduction}

a number of disadvantages: (1) erroneous readings from a single spacecraft have a drastic effect on the entire formation since state information cannot be obtained from other spacecraft in the formation, (2) severe computation loads imposed in the master filter resulting from global computation of state estimation and control as opposed to local computation at each spacecraft node, and (3) high communication overhead resulting from the demand of the master filter to have a complete state information of all spacecraft in the formation.

2. Distributed estimation architecture include an hierarchy of filters where each spacecraft runs its own local filter which provide estimates to the master filter then combines to local state estimation into formation state estimation. The principle of distributed estimation is to reduce computation loads of centralized estimation by distributing the spacecraft in formation flying among the hierarchy of local filters. This architecture has the advantage of distributing the computation loads among local spacecraft; however, it retain the disadvantages pertaining to centralized architectures.

3. Decentralized estimation architecture is non-hierarchical and eliminate the need for coordination by a central supervisor. Each spacecraft in a decentralized network processes only its own measurements data in parallel with the other spacecraft. Although, the total computational burden over the entire formation is greater than it would be for a monolithic spacecraft. Requirements for data transmission between spacecraft are minimized, at the cost of locally maintaining an additional data vector.

In this research, we focus on developing distributed nonlinear filters for state estimation in the presence of an unmeasurable states for a spacecraft in formation flying. 


\section{Chapter 4. Distributed Attitude Estimation and Control of Spacecraft}

Formation Flying

\subsubsection{Literature review}

Several estimation algorithms have been proposed to estimate the state of spacecraft formation flying (SFF); these include Kalman filter (KF), extended Kalman filter (EKF), unscented Kalman filter (UKF), and particle filters. The EKF found the largest number of applications in SFF domain. Carpenter [Carpenter 2002] proposed a decentralized architecture for autonomous establishment and maintenance of spacecraft formations using the EKF approach. In the reported work, local control has been achieved by processing local measurement information and transmission vectors from the other nodes. Kim et al. [Kim et al. 2007] formulated an EKF to estimate the relative attitude and position of two spacecraft using line-of-sight (LOS) measurements coupled with gyro measurements from each spacecraft to determine relative attitude, position and gyro biases. The attitude kinematics were described by the quaternion while general relative orbital equations were used to describe the position. Nebelecky et al. [Nebelecky et al. 2009] achieved a decentralized attitude estimation between three spacecraft through the use of a local EKF and data fusion process known as the Covariance Intersection algorithm. The EKF has been proposed for relative position and attitude estimation for spacecraft formation [Xing et al. 2010]. In this reported work, a position sensitive diode (PSD) sensor has been used to provide multiple line-of-sight (LOS) vectors from a feature point to beacons fixed on the leader spacecraft. The LOS measurements were coupled with spacecraft dynamics model and EKF to estimate relative attitude of the deputy and relative position of the feature point. Chen et al. [Chen et al. 2010] integrated measurements from Global Position System (GPS) and Vision based Navigation (VISNAV) to form a relative navigation and attitude determination system for ultra-close spacecraft formation flying. Federal Kalman Filter (FKF) architecture was adopted for the relative navigation and attitude determination states estimation and optimal fusion of GPS/VISNAV subsystem.

Implementing the extended Kalman filter may result in large errors in the true posterior mean and covariance of the transformed random variables since the estimation is based on linearization of the system dynamics, which results in a suboptimal application of the recursive estimation of the standard Kalman Filter. Given the limitation of the extended 


\subsection{Introduction}

Kalman filter in estimating the state of Spacecraft Formation Flying, an alternative filtering approach has been developed based on unscented transformation principles. The UKF has been proposed for relative spacecraft attitude and position estimation [Zhang et al. 2015]. In the reposted work, Zhang assumed a full state feedback in estimating the spacecraft attitude and position and implemented the Generalized Rodrigues Parameters (GRP) to maintain the quaternion normalization constraint. The performance of the UKF depends on the accuracy of the system model and on prior statistical knowledge of the process and measurement noise. Since, the UKF cannot adapt itself to deal with the uncertainty model errors and since the statistical characteristics of the noises are difficult to obtain, other alternatives have been proposed for estimating the state of Spacecraft Formation Flying. One of the approaches is the particle filtering. The UKF resembles the particle filter algorithm but with a major and extremely important difference. The samples are drawn in a specific and deterministic manner rather than at random as in the particle filter. All the research paper above have considered a full state feedback and none of the research paper above have considered the development of a distributed adaptive unscented Kalman filter and distributed particle filter to estimate the unmeasurable angular velocity of spacecraft in formation flying. 


\section{Chapter 4. Distributed Attitude Estimation and Control of Spacecraft}

Formation Flying

\subsubsection{Objectives}

Several nonlinear filtering techniques and control algorithms have been proposed to track a time-varying reference trajectory under the availability of full state measurements. However, in the open literature there has not been much emphasis placed on distributed estimation under the assumption of partial information exchange between spacecraft while some of the attitude states are unmeasurable. The main contributions of this study are as follows:

1. Estimation of unmeasurable angular velocities for SFF: An accurate nonlinear filtering techniques for spacecraft formation flying are investigated to estimate the unmeasurable angular velocity of a spacecraft which tracks a common-time varying reference attitude. Two nonlinear filtering techniques are investigated herein, the adaptive unscented Kalman filter (AUKF) and particle filter (PF). Six spacecraft are involved in the formation including one virtual leader and only measurements of spacecraft attitude are available for use where the attitude of is represented by modified Rodrigues parameters (MRP).

2. Estimation of reference angular acceleration of a virtual leader in SFF: A distributed filter is developed for estimating the reference angular acceleration of a virtual leader in spacecraft formation flying. The filter is developed such that the attitude estimations of all spacecraft in the formation can track time-varying reference trajectory even in the absence of angular velocity measurements. Distributed filter is required since the virtual leader exchange only partial information with other spacecraft in formation. Here, we consider the case when the common time-varying reference attitude is available only to a subset of the spacecraft in formation.

3. Performance evaluation: Performance evaluation and comparison between AUKF and PF for online estimation under faults in the dynamic models, control laws, and measurement models. The performance comparison is illustrated with numerical simulation through calculations of formation-keeping error and station-keeping error. 


\subsection{System Model, Filter Model, and Control Law}

In this section, dynamic equations, kinematic equations, filter models, and control laws are constructed for spacecraft formation flying. Distributed filtering algorithms are investigated for a group of spacecraft in formation where only measurements of spacecraft attitude are available and the virtual leader exchanges state information with a subset of the group members. Filters are constructed such that all spacecraft in formation can simultaneously track a time varying reference attitude. Figure 5.1 shows the communication graph for spacecraft formation.

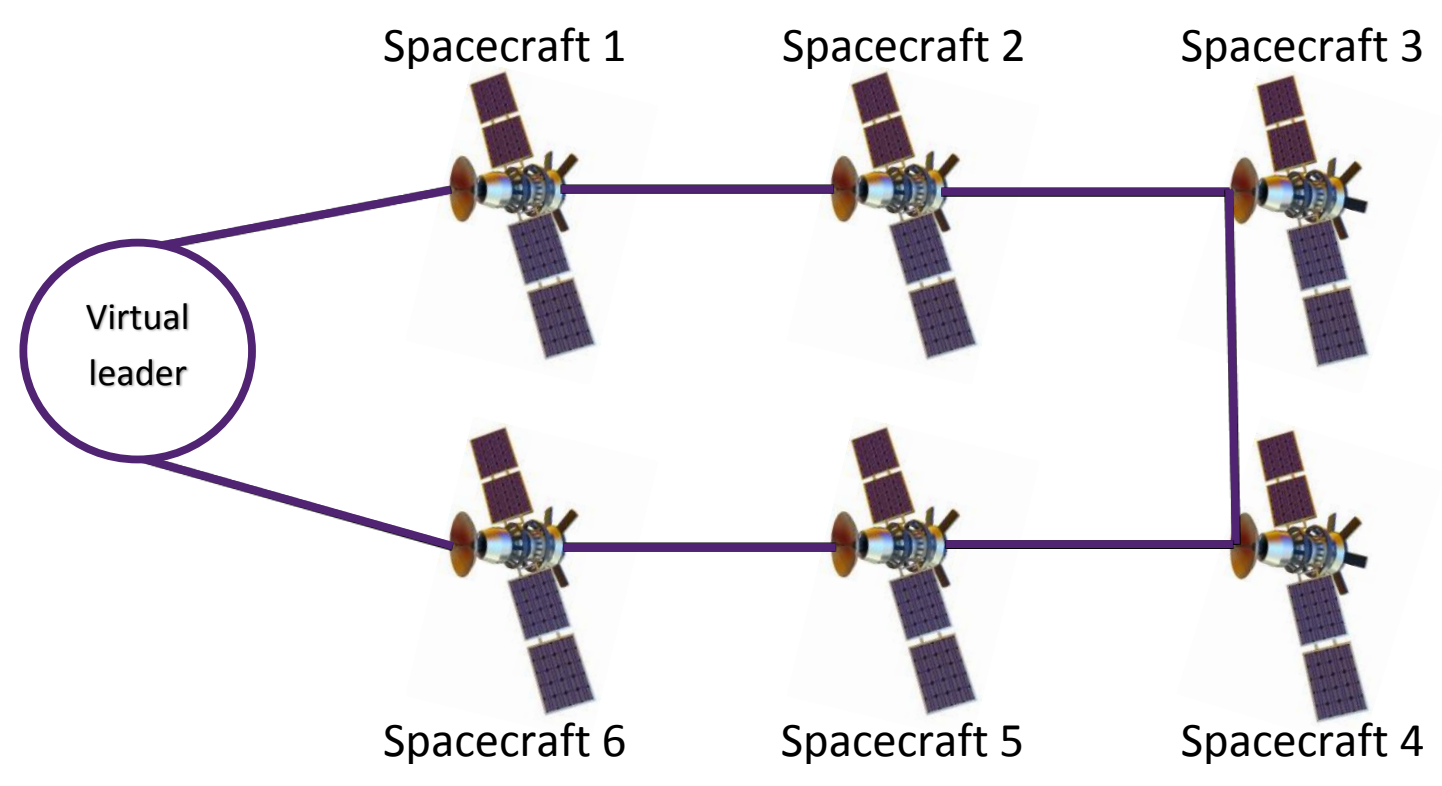

Figure 4.1: Communication graph for spacecraft formation 


\section{Chapter 4. Distributed Attitude Estimation and Control of Spacecraft}

Formation Flying

\subsubsection{Dynamic equations}

For a group of $n$ spacecraft, the individual spacecraft is modeled as a rigid body with the following dynamic equation for the $i$ th $(i=1,2, \cdots, n)$ spacecraft [Hughes 1986]

$$
J_{i} \dot{\omega}_{i}=-\omega_{i}^{\times} J_{i} \omega_{i}+\tau_{i}
$$

where $J_{i} \in R^{3 \times 3}$ is the mass moment-of-inertia matrix, $\tau_{i} \in R^{3}$ is the torque control, $\omega_{i} \in R^{3}$ is the angular velocity of the $i$ th spacecraft in a body-fixed frame, and $x^{\times} \in R^{3 \times 3}$ denotes the skew-symmetric matrix given by

$$
x^{\times}=\left[\begin{array}{ccc}
0 & -x_{3} & x_{2} \\
x_{3} & 0 & -x_{1} \\
-x_{2} & x_{1} & 0
\end{array}\right]
$$

\subsubsection{Kinematic equations}

For a group of $n$ spacecraft, the kinematic equation of the $i$ th $(i=1,2, \cdots, n)$ spacecraft in formation is given by [Hughes 1986]

$$
\dot{q}_{i}=T_{i}\left(q_{i}\right) \omega_{i}
$$

where $\omega_{i} \in R^{3}$ is the angular velocity of the $i$ th spacecraft in a body-fixed frame, $q_{i}(t) \in$ $R^{3}$ represents the Modified Rodriguez Parameters (MRPs) which describe the spacecraft attitude with respect to an inertial frame [Shuster 1993]

$$
q_{i}(t)=\varrho_{i}(t) \tan \left(\frac{\phi_{i}(t)}{4}\right), \quad \phi_{i} \in[0,2 \pi) \mathrm{rad}
$$

where $\varrho_{i}$ and $\phi_{i}$ denote the Euler eigenaxis and eigenangle, respectively. The matrix $T_{i}\left(q_{i}\right) \in$ $R^{3 \times 3}$ for MRPs is given by [Hughes 1986]

$$
T_{i}\left(q_{i}\right)=\frac{1}{2}\left[\frac{1-q_{i}^{T} q_{i}}{2} I_{3}+q_{i}^{\times}+q_{i} q_{i}^{T}\right] .
$$

The spacecraft models in Equations (4.1) and (4.3) can be transformed as follows [Zou 2014]

$$
\begin{aligned}
\dot{q}_{i} & =\mathrm{v}_{i} \\
\dot{\mathrm{v}}_{i} & =f_{i}\left(q_{i}, \mathrm{v}_{i}\right)+g_{i}\left(q_{i}\right) \tau_{i}
\end{aligned}
$$

For $i=1,2, \cdots, n$, where the function $f_{i}\left(q_{i}, \mathrm{v}_{i}\right)=-T_{i} \dot{P}_{i} \dot{q}_{i}-T_{i} J_{i}^{-1}\left(P_{i} \dot{q}_{i}\right)^{\times} J_{i} P_{i} \dot{q}_{i}, P_{i}=$ $T_{i}^{-1}\left(q_{i}\right)$, and $g_{i}=T_{i} J_{i}^{-1}$. 


\subsection{System Model, Filter Model, and Control Law}

\subsubsection{Filter models}

Spacecraft filter model: Each of the $n$ spacecraft runs a filter to estimate the posterior distribution of the spacecraft attitude and unmeasurable angular velocity. The estimated attitude motion and angular velocities are computed with the following model

$$
\hat{\mathrm{x}}_{k+1}=f\left(\hat{\mathrm{x}}_{k}, \tau_{k}\right)+w_{k}
$$

where $\hat{\mathrm{x}}_{k}$ is the state vector, $\tau_{k}$ is the control torque, and $w_{k}$ is the additive process noise. The notation $(\hat{\cdot})$ denotes the estimation of $(\cdot)$. The function $f\left(\hat{\mathrm{x}}_{k}, \tau_{k}\right)$ is propagated through the forth-order Runge-Kutta method as given by Equation (3.7) and Equation (3.8), where the function $G(\hat{\mathrm{x}})$ is given as follows:

$$
G(\hat{\mathrm{x}})=\left[\begin{array}{c}
\dot{\hat{q}}_{i} \\
\dot{\hat{\mathrm{v}}}_{i}
\end{array}\right] \quad i=1,2, \cdots, n
$$

where $\dot{\hat{q}}_{i}=\hat{\mathrm{v}}_{i}$ and $\dot{\hat{\mathrm{v}}}_{i}=f_{i}\left(q_{i}, \hat{\mathrm{v}}_{i}\right)+g_{i}\left(q_{i}\right) \tau_{i}$, note that $\hat{q}_{i}$ and $\hat{\mathrm{v}}_{i}$ are estimates of $q_{i}$ and $\mathbf{v}_{i}$, respectively, and $f_{i}\left(q_{i}, \hat{\mathrm{v}}_{i}\right)=-T_{i} \dot{P}_{i} \dot{q}_{i}-T_{i} J_{m, i}^{-1}\left(P_{i} \dot{q}_{i}\right)^{\times} J_{m, i} P_{i} \dot{q}_{i}$ with the following parameters $P_{i}=T_{i}^{-1}\left(\hat{q}_{i}\right), g_{i}=T_{i} J_{i}^{-1}$, and $J_{m} \in R^{3 \times 3}$ is the positive-definite mass moment-of-inertia matrix of the filter model. The vector $q$ denotes the modified Rodrigues parameters (MRP) and is obtained directly from the measurement model $y_{k}$ which is given by

$$
y_{k}=\left[q_{k}\right]+\left[v_{q k}\right]
$$

Distributed filter model: The virtual leader exchanges partial information with other spacecraft in the formation and only the neighboring spacecraft have access to information. A distributed model is incorporated to estimate the reference angular acceleration of the $i^{\text {th }}$ SFF. The proposed distributed model is propagated through forth-order Runge-Kutta method similar to the procedure in Equations (3.7) and (3.8),

$$
\dot{p}_{i}=\phi\left[\sum_{j=1}^{n} a_{i j}\left(p_{i}-p_{j}\right)+a_{i 0}\left(p_{i}-\dot{\mathrm{v}}_{0}\right)\right]
$$

where $p_{i}$ is an estimation of $\dot{\mathrm{v}}_{0}$ where $p_{i}(0)=0, \phi$ is a constant parameter, $a_{i j}$ is an element of the weighted adjacency matrix $A$ which denotes the communication quality between the 


\section{Chapter 4. Distributed Attitude Estimation and Control of Spacecraft}

Formation Flying

$i^{t h}$ and $j^{t h}$ spacecraft. The weighted adjacency element $a_{i j}(i=1,2, \cdots, n, j=1,2, \cdots, n)$ is bounded, such that, $a_{i i}=0$ and $0<a_{i j}<1$. The parameter $a_{i 0}(i=1,2, \cdots, n)$ is a constant which defines the adjacency matrix $B=\operatorname{diag}\left\{a_{10}, a_{20}, \cdots, a_{i 0}\right\}$. The element $a_{i 0}>0$ if the $i$ th spacecraft has access to the leader, otherwise $a_{i 0}=0$.

\subsubsection{Control law}

The distributed asymptotic attitude coordination control law for a group of spacecraft is given by [Zou et al. 2016]

$$
\tau_{i}=g_{i}^{-1}\left[-k_{1}^{2} k_{2} \hat{\chi}_{1 i}-k_{1} k_{3} \hat{\chi}_{2 i}-f_{i}\left(q_{i}, \hat{\mathrm{v}}_{i}\right)-\theta^{2} \beta \tilde{q}_{i}+p_{i}\right]
$$

For $i=1,2, \cdots, n$, where the parameters $k_{1}, k_{2}, k_{3}, \theta$, and $\beta$ are some positive constants, $p_{i}$ is an estimation of $\dot{\mathrm{v}}_{0}$, and $\hat{\chi}_{1 i}$ and $\hat{\chi}_{2 i}$ are the estimated lumped attitude state errors including station-keeping and formation-keeping errors for the $i$ th spacecraft in formation. The parameters $\hat{\chi}_{1 i}$ and $\hat{\chi}_{2 i}$ are defined as

$$
\begin{aligned}
& \hat{\chi}_{1 i}=\sum_{j=1}^{n} a_{i j}\left(\hat{q}_{i}-\hat{q}_{j}\right)+a_{i 0}\left(\hat{q}_{i}-q_{0}\right) \\
& \hat{\chi}_{2 i}=\sum_{j=1}^{n} a_{i j}\left(\hat{\mathrm{v}}_{i}-\hat{\mathrm{v}}_{j}\right)+a_{i 0}\left(\hat{\mathrm{v}}_{i}-\mathrm{v}_{0}\right)
\end{aligned}
$$

where $\mathrm{v}_{0}=\dot{q}_{0}$, and $i=1,2, \cdots, n$. The control law depends on attitude, velocity and acceleration estimations and on the state of the virtual leader (i.e., $q_{0}, \mathrm{v}_{0}$, and $\dot{\mathrm{v}}_{0}$ ) if and only if the $i$ th spacecraft is a neighbor of the virtual leader.

\subsection{Simulation Results and Discussion}

Numerical simulations are presented to verify the performance of the online distributed estimation under fault-free and fault-prone scenarios. The faults may occur with the system dynamics, control command, or measurement models. Here, the adaptive unscented Kalman filter (Chapter 1.4.3) and particle filter (Chapter 1.4.4) are implemented to obtain an online distributed estimation of unmeasurable angular velocity. The problem addressed in the present work is challenging given the fact that not all spacecraft in the formation 


\subsection{Simulation Results and Discussion}

have direct access to the virtual leader. The virtual leader, labeled as spacecraft 0 , has states given by a time-varying reference trajectory for the spacecraft formation. The forth-order Runge-Kutta method was implemented to numerically integrate the system model and filter models with an integration time step $t=0.01 \mathrm{sec}$ and $t=0.01 \mathrm{sec}$ for a simulation time of $T_{\text {sim }}=200 \mathrm{sec}$.

System parameters: The communication quality between spacecraft is expressed with the weighted adjacency matrix ${ }^{2} A=\left(a_{i j}\right)_{6 \times 6}$, where $a_{12}=a_{21}=0.4, a_{16}=a_{61}=0.6$, $a_{23}=a_{32}=0.6, a_{34}=a_{43}=0.6, a_{45}=a_{54}=0.8, a_{56}=a_{65}=0.6$, and $a_{i j}=0$ for all other elements. The symmetric weighted adjacency matrix $a_{i 0}>0(i=1,2, \ldots, n)$ is a constant if the $i$ th spacecraft has access to the virtual leader, otherwise $a_{i 0}=0$. The adjacency matrix $B=\operatorname{diag}[0.5,0,0,0,0,0.5]$. The reference attitude is $q_{0}=0.2[\cos (0.2 t), \sin (0.2 t), \sqrt{3}]^{T}$ and the external disturbances are assumed as $\vartheta_{i}=0.1[\sin (i t), \cos (i t), \sin (2 i t)]^{T}$. Table 4.1 shows the initial attitude and angular velocities, and Table 4.2 present the mass moment of inertia of each spacecraft [Zou et al. 2016].

Table 4.1: Initial attitude and angular velocity.

\begin{tabular}{|c|l|c|}
\hline \hline Spacecraft & Initial attitude & Initial angular velocity \\
\hline \hline 1 & $q_{1}(0)=[0,1, \sqrt{3}]^{T}$ & $\omega_{1}(0)=0$ \\
\hline 2 & $q_{2}(0)=-0.4[1,1, \sqrt{2}]^{T}$ & $\omega_{2}(0)=0$ \\
\hline 3 & $q_{3}(0)=1.4[\sqrt{3}, 1,0]^{T}$ & $\omega_{3}(0)=0$ \\
\hline 4 & $q_{4}(0)=-0.6[\sqrt{3}, 0,1]^{T}$ & $\omega_{4}(0)=0$ \\
\hline 5 & $q_{5}(0)=1.5[1, \sqrt{2}, 1]^{T}$ & $\omega_{5}(0)=0$ \\
\hline 6 & $q_{6}(0)=-1.2[1,1, \sqrt{2}]^{T}$ & $\omega_{6}(0)=0$ \\
\hline \hline
\end{tabular}

Control parameters: The saturation limit of the control law is $\left|\tau_{i j}\right| \leq 0.1 \mathrm{Nm}$, where $i=1,2, \cdots, n, j=1,2,3$, and the control parameters are $\beta=2, \theta=10, k_{1}=0.5, k_{2}=15$, and $k_{3}=20$.

\footnotetext{
${ }^{2}$ The weighted adjacency matrix $A$ is a symmetric matrix (i.e., $a_{i j}=a_{j i}$ and $a_{i i}=0$ ) with the adjacency elements $a_{i j}$ which denotes the communication quality between the $i$ th and $j$ th spacecraft, $\left(i . e ., a_{i j}>0\right)$.
} 


\section{Chapter 4. Distributed Attitude Estimation and Control of Spacecraft}

Formation Flying

Filter parameters: The standard deviation of the process noise and measurement noise are $\sigma_{Q}=1 \times 10^{-3}$, and $\sigma_{R}=1 \times 10^{-4}$. The covariance matrix of process noise and measurement noise are given by $Q=E\left\{w w^{T}\right\}=\sigma_{Q}^{2} I_{n \times n}$, and $R=E\left\{v v^{T}\right\}=\sigma_{R}^{2} I_{m \times m}$.

Adaptive unscented Kalman filter: The scaling parameter ${ }^{3} \lambda=\alpha_{A U K F}^{2} \times(n+\kappa)-n$, where $\alpha_{A U K F}=0.1, \beta_{A U K F}=2, \kappa=0$, and $n$ is the dimension of the augmented state. The filter weights are $\mathrm{W}_{0}^{(m)}=\lambda /(n+\lambda), \mathrm{W}_{0}^{(c)}=\lambda /(n+\lambda)+\left(1-\alpha_{A U K F}^{2}+\beta_{A U K F}\right)$, and $\mathrm{W}_{i}^{(m)}=\mathrm{W}_{i}^{(c)}=1 / 2(n+\lambda)$, for $i=1,2, \ldots, n$ where $\mathrm{W}_{i}^{(m)}$ and $\mathrm{W}_{i}^{(c)}$ are the weight associated with the mean and covariance matrices. The fading factor in AUKF is $\zeta=1 \times 10^{3}$.

Particle filter: The number of particles $N_{p}=100$, and the resampling threshold for Effective Sample Size (ESS), $\beta_{P F}=0.8$. The systematic resampling algorithm was selected, see [Carpenter et al. ], [Kitagawa 1996], or [Chen 2003], and the proposal distribution was selected such that

$$
q\left(\mathrm{x}_{k} \mid \mathrm{x}_{k-1}, \mathrm{y}_{1: k-1}\right)=\mathcal{N}\left(\mathrm{x}_{k} ; f\left(\mathrm{x}_{k-1}\right), Q\right)
$$

where $\mathcal{N}\left(\mathrm{x}_{k} ; f\left(\mathrm{x}_{k-1}\right), Q\right)$ is the multivariate Gaussian distribution with expected value and variance. See [Haug 2005] for details on the proposal distribution.

Attitude error matrices: Performance comparison, between adaptive unscented Kalman filter and particle filter, was investigated based on station-keeping and formation-keeping attitude error metrics. The station-keeping attitude error (SKAE) metric is defined as

$$
\mathrm{SKAE}=\sqrt{\sum_{i=1}^{6}\left\|e_{1 i}\right\|^{2}}
$$

and the formation-keeping attitude error (FKAE) metric is defined as

$$
\mathrm{FKAE}=\sqrt{\sum_{i=1}^{6} \sum_{j=i+1}^{6}\left\|q_{i}-q_{j}\right\|^{2}}
$$

The station-keeping attitude error (SKAE) and formation-keeping attitude error (FKAE) are used as error metrics for all fault-prone scenarios in this simulation.

\footnotetext{
${ }^{3}$ The composite scaling parameter $\lambda$ is a function of three variables: (1) $\alpha_{A U K F}$ which determines the spread of the sigma points around the mean $\overline{\mathbf{x}},(2) \beta_{A U K F}$ which incorporate prior knowledge of the distribution of the state $\mathbf{x}$, and $(3) \kappa$ which is a secondary scaling parameter usually set to 0 .
} 


\subsection{Simulation Results and Discussion}

\subsubsection{Fault-free spacecraft formation flying}

Six spacecraft are involved in the formation including one virtual leader. The spacecraft are modeled as rigid bodies, where the mass moment of inertia tensor for each Spacecraft is presented in Table (4.2) [Ren 2007]. The mass moment of inertia tensor of the filter is given in Table (4.3). The purpose of this study is to compare the performance of the adaptive unscented Kalman filter and particle filter when no faults are introduced.

Table 4.2: Mass moment-of-inertia tensor for each spacecraft in the formation.

\begin{tabular}{|c|c|}
\hline Spacecraft & Inertia matrix $\left[\mathrm{kg} \cdot \mathrm{m}^{2}\right]$ \\
\hline 1 & 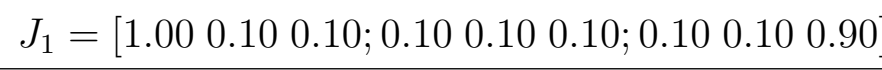 \\
\hline 2 & 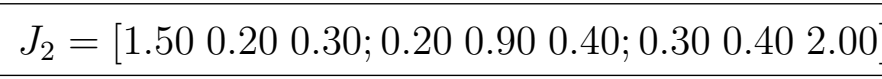 \\
\hline 3 & 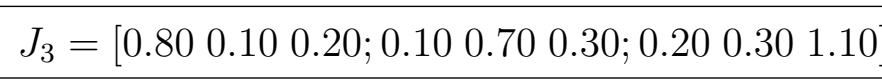 \\
\hline 4 & 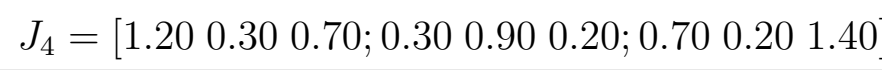 \\
\hline 5 & 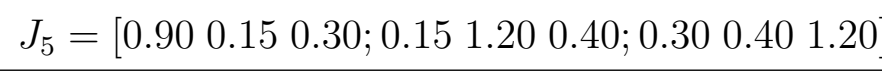 \\
\hline 6 & 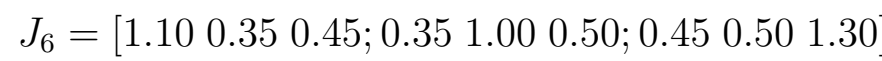 \\
\hline
\end{tabular}

Table 4.3: Filter mass moment-of-inertia tensor for each spacecraft in the formation.

\begin{tabular}{|c|c|}
\hline Spacecraft & Inertia matrix $\left[\mathrm{kg} \cdot \mathrm{m}^{2}\right]$ \\
\hline 1 & 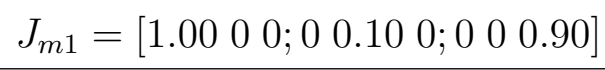 \\
\hline 2 & 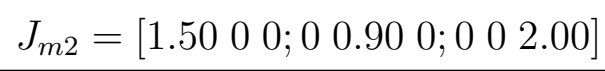 \\
\hline 3 & $J_{m 3}=\left[\begin{array}{lllllll}0.80 & 0 & 0 & 0 & 0.70 & 0 & 0\end{array}\right]$ \\
\hline 4 & 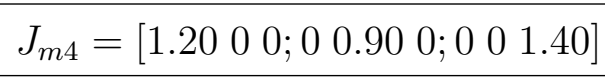 \\
\hline 5 & 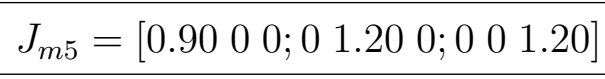 \\
\hline 6 & 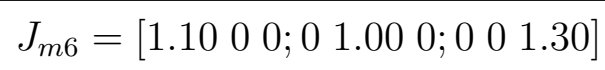 \\
\hline
\end{tabular}




\section{Chapter 4. Distributed Attitude Estimation and Control of Spacecraft}

Formation Flying

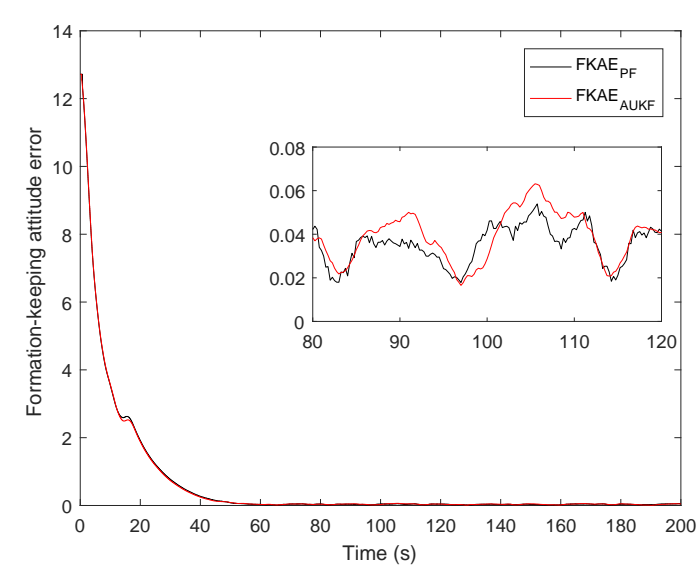

(a) FKAE

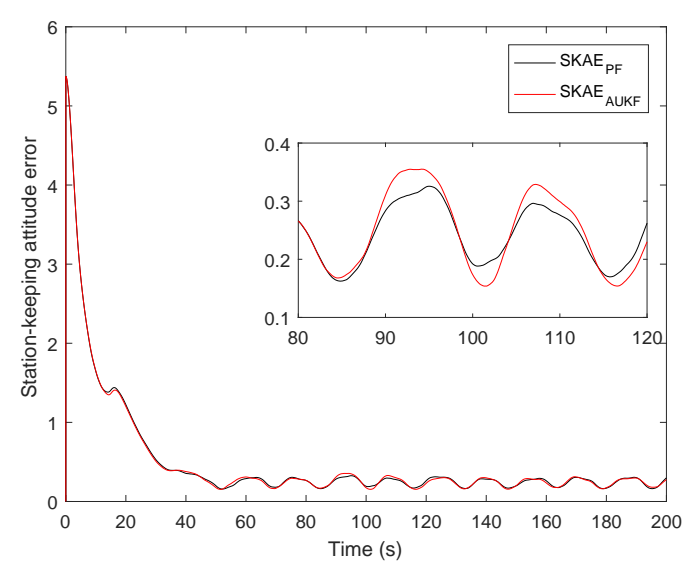

(b) SKAE

Figure 4.2: Formation-keeping and station-keeping attitude errors.

Figure 4.2(a) presents the formation-keeping attitude error and Figure 4.2(b) shows the station-keeping attitude error. Under no fault condition, the particle filter estimates the spacecraft states with higher accuracy than AUKF resulting in a faster convergence of formation-keeping error and station-keeping errors. Here, performance of particle filter are possible due to small number of particles selected for practical application. As the number of particles drawn from the posterior distribution increases, better approximation of the intractable integrals appearing in the Bayesian equation is possible at the expense of computation time. The small number of particles selected herein is sufficient to approximate the continuous distributions and estimate the unknown hidden attitude states of the Spacecraft in formation flying. 


\subsection{Simulation Results and Discussion}

\subsubsection{Uncertainty in moment-of-inertia and external disturbances}

In the following section, faults are introduces in the spacecraft dynamics with the purpose of comparing the accuracy of online distributed estimation between the adaptive unscented Kalman filter and particle filter. Ideally, the evaluation will be based on the capacity to estimate the attitude states and maintain a specified station keeping and formation keeping. Two possible scenarios are considered: In the first scenario, uncertainty is introduced in the mass moment-of-inertia tensor. In the second scenario, external disturbances are introduced.

\section{Case I: Uncertainty in Moment of Inertia}

In the following case, uncertainty is introduced in the mass moment-of-inertia (MOI) tensor. The uncertainty in MOI reflects a fault that may occur in the Spacecraft dynamics. Changes in MOI are compensated by the control command which is driven by the estimated states i.e. attitude response and angular velocity. Four faults are introduced at Spacecraft during a simulation time of 200-seconds. Initially, no fault occur at the Spacecraft dynamics and the MOI corresponds to the MOI in Table (4.2). The first fault occur 10-seconds after the start of simulation for a duration of 40-seconds. Here, the magnitude of uncertainty in MOI (i.e. $I_{x x}, I_{y y}$ and $I_{x x}$ ) increase significantly, while the product of inertia (i.e. $I_{x y}, I_{x z}, I_{y z}$ etc.) remain unchanged. The second fault occur at 50-seconds for a duration of 30-seconds. During the fault, the magnitude of uncertainty in MOI decreases by 50 percent from the fault at previous interval. Finally, the third fault occur at 80-seconds and remains until the end of the simulation. The Uncertainty in MOI is calculated as $J_{\text {Fault }}=J_{i}+J_{k}$ $(i=1,2, \cdots, n)$ where $J_{i}$ is the mass moment-of-inertia tensor presented in Table (4.2), and $J_{k}$ is the uncertainty tensor as shown in Table (4.4).

Figure 4.3(a) shows the formation-keeping attitude error and Figure 4.3(b) shows the station-keeping attitude error. It is interesting to note that the uncertainty in mass moment-of-inertia tensor effect the formation-keeping attitude error (FKAE) and station -keeping attitude error (SKAE). Initially, the convergence rate of the adaptive unscented Kalman filter is slightly faster than the particle filter. This is because of computation time required by the particle filter to estimate the states and establish formation-keeping 


\section{Chapter 4. Distributed Attitude Estimation and Control of Spacecraft}

Formation Flying

Table 4.4: Uncertainty in moment-of-inertia.

\begin{tabular}{|c|c|}
\hline Time [sec] & Uncertainty in MOI tensor $\left[\mathrm{kg} \cdot \mathrm{m}^{2}\right]$ \\
\hline $0<t \leqslant 10$ & 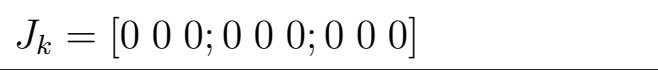 \\
\hline $10<t \leqslant 50$ & 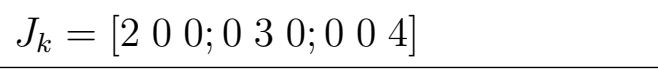 \\
\hline $50<t \leqslant 80$ & 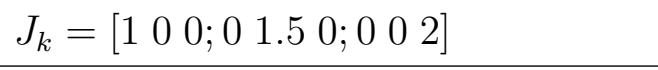 \\
\hline $80<t \leqslant 100$ & 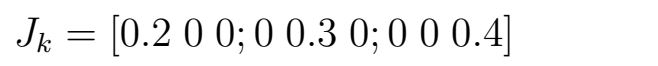 \\
\hline
\end{tabular}

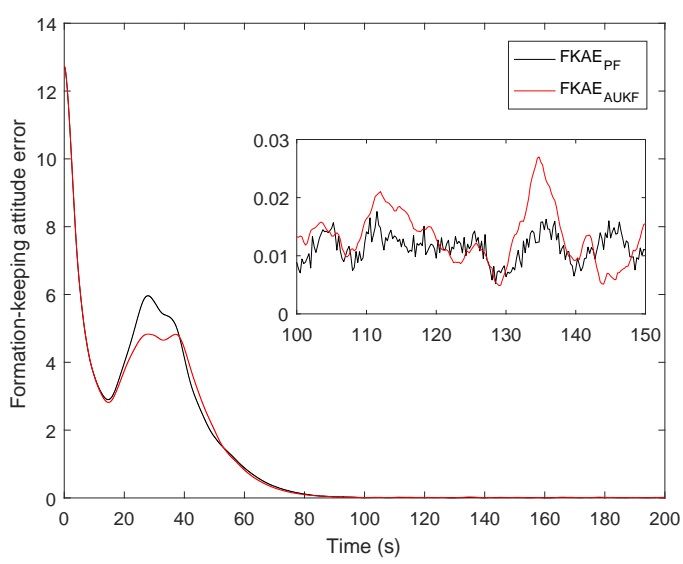

(a) Formation-Keeping Attitude Error

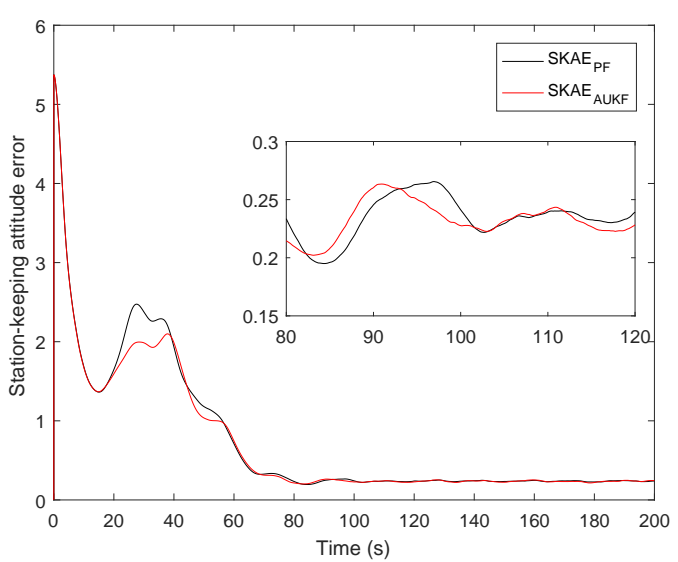

(b) Station-Keeping Attitude Error

Figure 4.3: Uncertainty in moment-of-inertia tensor.

and station-keeping. Once uncertainty in MOI decreases, the adaptive unscented Kalman filter approximate the hidden states to higher accuracy and the formation-keeping error and station-keeping error are reduced. 


\subsection{Simulation Results and Discussion}

\section{Case II: External disturbances}

In the following case, the spacecraft in formation experience external disturbances acting on each one of the principal axes. External disturbances $\vartheta_{i}$ for $(i=1,2, \cdots, n)$ are added to the $i t h$ spacecraft dynamic equation (Equation 4.1 ) which result in a modified dynamic equation

$$
J_{i} \dot{\omega}_{i}=-\omega_{i}^{\times} J_{i} \omega_{i}+\tau_{i}+\vartheta_{i}
$$

where the magnitude of the external disturbances is given in Table (4.5). The external disturbances are introduced at four different time intervals during the simulation. At the start of the simulation, external disturbances equivalent to $\vartheta_{i}=$ $[0.25 \sin (t) ; 0.25 \cos (t) ; 0.25 \sin (2 t)]$ are introduced at the spacecraft dynamic model for $10-$ seconds. The second interval starts at $t=10$-second when external disturbances equivalent to $\vartheta_{i}=[0.5 \sin (t) ; 0.5 \cos (t) ; 0.5 \sin (2 t)]$ are applied to the spacecraft dynamic model for a duration of 15 -seconds. The third interval starts at $t=25$-second when the magnitude of external disturbances equivalent to $\vartheta_{i}=[0.1 \sin (t) ; 0.1 \cos (t) ; 0.1 \sin (2 t)]$ are applied for a duration of 25-seconds. Finally, the last interval starts at $t=50$-seconds where external disturbances are equivalent to $\vartheta_{i}=[0.01 \sin (t) ; 0.01 \cos (t) ; 0.01 \sin (2 t)]$.

Table 4.5: External disturbances.

\begin{tabular}{|l|l|}
\hline \hline Time $[\mathrm{sec}]$ & External disturbance \\
\hline \hline $0<t \leqslant 10$ & $\vartheta_{i}=d_{k}[\sin (t) ; \cos (t) ; \sin (2 t)]$ where $d_{k}=0.25$ \\
\hline $10<t \leqslant 25$ & $\vartheta_{i}=d_{k}[\sin (t) ; \cos (t) ; \sin (2 t)]$ where $d_{k}=0.5$ \\
\hline $25<t \leqslant 50$ & $\vartheta_{i}=d_{k}[\sin (t) ; \cos (t) ; \sin (2 t)]$ where $d_{k}=0.1$ \\
\hline $50<t \leqslant 100$ & $\vartheta_{i}=d_{k}[\sin (t) ; \cos (t) ; \sin (2 t)]$ where $d_{k}=0.01$ \\
\hline \hline
\end{tabular}




\section{Chapter 4. Distributed Attitude Estimation and Control of Spacecraft}

Formation Flying

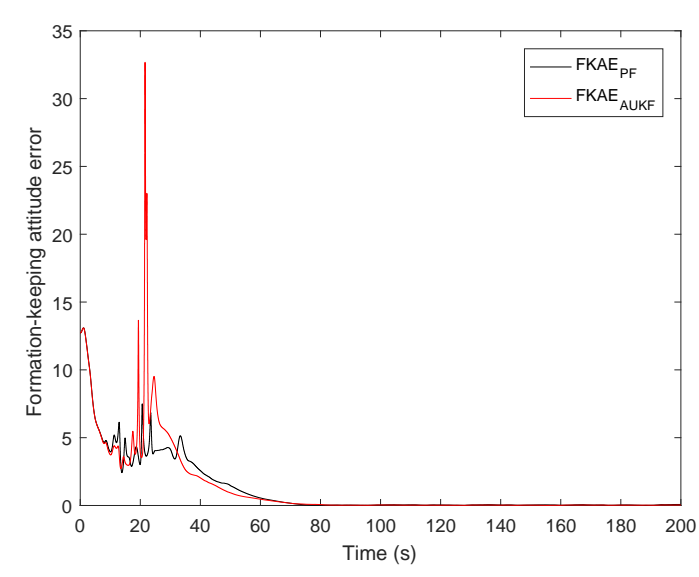

(a) Formation-Keeping Attitude Error

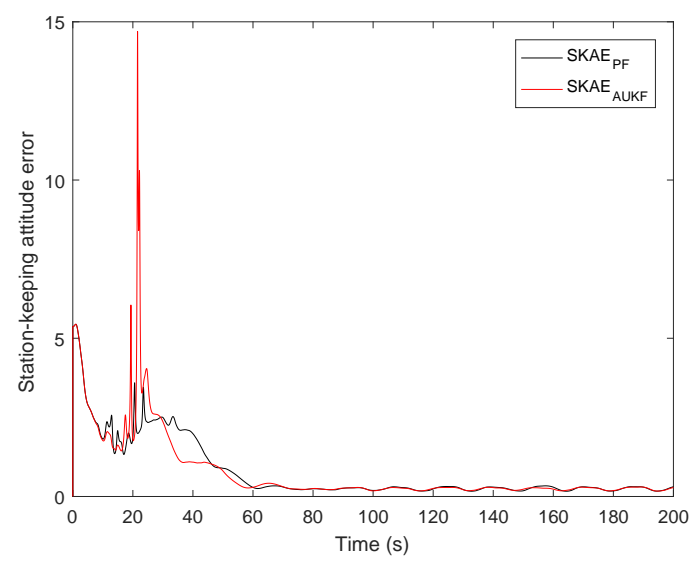

(b) Station-Keeping Attitude Error

Figure 4.4: External disturbances.

Figure 4.4(a) shows the formation-keeping attitude error and Figure 4.4(b) shows the station-keeping attitude error for spacecraft in formation flying in the presence of external disturbance. The figure shows the adaptive unscented Kalman filter experienced significantly large formation-keeping error and station keeping error in the presence of external disturbance. However, the effect of the external disturbance was not significantly apparent in the particle filter. These significantly large errors in formation-keeping and stationkeeping are directly impacted by the small number of sigma points used to estimate the posterior distribution of the states. Recall: the number of sigma points (sampling points) in AUKF is given by $2 N_{x}+1$ whereas the number of sampling points selected for the particle filter is $N_{p}=100$. Small number of sampling points of AUKF is not sufficient for state estimation when the rate-of-change is large. For large rate-of-change the particle filter can estimate the posterior distribution to higher accuracy and lower the formation-keeping and station keeping errors. In the case of AUKF, formation-keeping error and station keeping error converge over a longer simulation time in comparison to particle filter. 


\subsection{Simulation Results and Discussion}

\subsubsection{Control command faults}

In the following section, fault are introduced at the control command with the purpose of comparing the accuracy of estimation between adaptive unscented Kalman filter and particle filter with the scope of reducing the station-keeping and formation-keeping errors under the influence of faults in the control command. Two cases are considered: In the first case, various magnitudes of control commands are applied at different time intervals. In the second case, there are no applied control commands during formation stabilization.

\section{Case I: Constant applied control commands}

In the following case, the distributed spacecraft in formation flying experience changes in the applied control commands as expressed in varying control signals and changes in control gains during the simulation time. The simulation is divided into four intervals for a duration of 200-seconds. Table (4.6) presents the time interval of fault occurrences and the corresponding applied control command. In the first interval, a constant control command is applied where $\tau_{1}, \tau_{3}$ and $\tau_{6}$ receive one control command while $\tau_{2}, \tau_{4}$ and $\tau_{5}$ receive a different control command. The seconds interval starts at $t=10$-seconds when the control command generates a constant control signal to only several spacecraft in the formation with no regards to system dynamics. This fault continuous until $t=15$-seconds where $\tau_{1}, \tau_{3}$, and $\tau_{6}$ are constant while $\tau_{2}, \tau_{4}$ and $\tau_{5}$ correspond to applied control law as per Equation (4.11). The third interval start at $t=15$-seconds until $t=25$-seconds. At this interval faulty applied control command were generated as a result of changes in control gains. The last interval start at $t=25$-seconds to the end of simulation. At this interval, the control command has been recovered and no fault is present. 


\section{Chapter 4. Distributed Attitude Estimation and Control of Spacecraft}

Formation Flying

Table 4.6: Constant applied control commands.

\begin{tabular}{|l|l|}
\hline \hline Time $[\mathrm{sec}]$ & Control command $\tau_{i}(i=1,2, \ldots, 6)$ \\
\hline \hline $0<t \leqslant 10$ & $\tau_{i}= \begin{cases}{[0.050 ; 0.025 ; 0.075],} & \text { for } i=1,3,6 \\
{[0.070 ; 0.050 ; 0.025],} & \text { for } i=2,4,5\end{cases}$ \\
\hline $10<t \leqslant 15$ & $\tau_{i}=\left\{\begin{array}{l}{[0.050 ; 0.025 ; 0.075],} \\
\text { According to Eqn. }(4.11), \quad \text { for } i=2,4,5\end{array}\right.$ \\
\hline $15<t \leqslant 25$ & $\tau_{i}=$ According to Eqn. $(4.11)\left(k_{1}=1.25, k_{2}=5, k_{3}=10\right)$ \\
\hline $25<t \leqslant 200$ & $\tau_{i}=$ According to Eqn. $(4.11)$ \\
\hline \hline
\end{tabular}

Figure4.5(a) show the formation-keeping attitude error and Figure4.5(b) show the station-keeping attitude error for SFF in the of presence different magnitudes of control commands. In the presence of different applied control commands, the AUKF and PF estimate the spacecraft state to the same accuracy resulting in similar convergence of formation-keeping error and station-keeping errors. However, once the faulty condition has recovered, the particle filter converge much faster than the AUKF. 


\subsection{Simulation Results and Discussion}

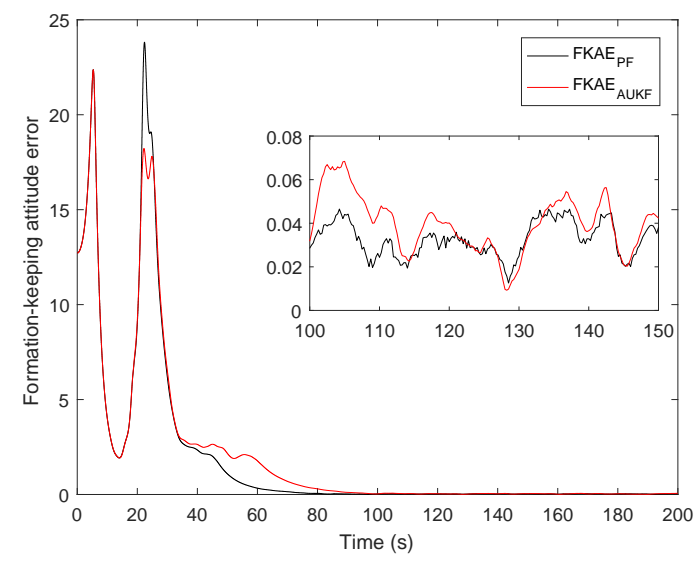

(a) Formation-Keeping Attitude Error

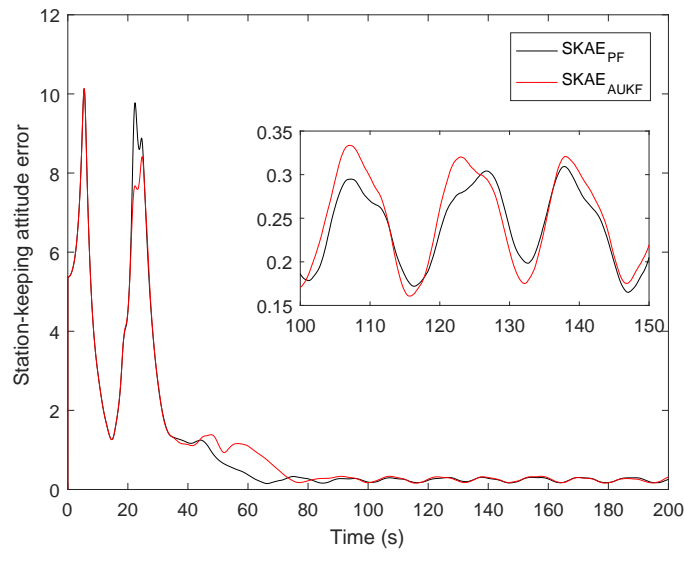

(b) Station-Keeping Attitude Error

Figure 4.5: Constant applied control commands.

\section{Case II: No control command during formation stabilization}

In the following case, several spacecraft in formation receive no control command during formation stabilization and once control command has been recovered the control parameters are changing. The simulation is divided into four intervals for a duration of 200-seconds. The first interval starts at $t=0$-seconds and continues for 20 -seconds. At this interval, the control command does not generate a torque signal for $\tau_{1}, \tau_{3}$ and $\tau_{6}$ while $\tau_{2}, \tau_{4}$ and $\tau_{5}$ receive a constant command. The second interval starts at $t=20$ continues for 20 -seconds. At this interval, the control command has been fully recovered, i.e., fault-free scenario, and the control command corresponds to Equation (4.11). The third interval, starts at $t=40$-seconds and continues for 30-seconds. Here, control parameters change as a result of faulty control command. Finally, the last interval start at $t=70$-seconds and continues until the end of simulation. In this interval, the control command has been recovered and no fault is present. Table (4.7) present the fault time and corresponding applied control command.

Figure 4.6(a) show the formation-keeping attitude error and Figure 4.6(b) show the station-keeping attitude error for Spacecraft in formation flying in the absence of applied control torque. The control command in Eqn. (4.11) is quite sensitive to the estimated 


\section{Chapter 4. Distributed Attitude Estimation and Control of Spacecraft}

Formation Flying

Table 4.7: No control command during formation stabilization.

\begin{tabular}{|l|l|}
\hline \hline Time $[\mathrm{sec}]$ & Torque magnitude $\tau_{i}(i=1,2, \ldots, 6)$ \\
\hline \hline $0<t \leqslant 20$ & $\tau_{i}=\left\{\begin{array}{l}{[0 ; 0 ; 0],} \\
{[0.050 ; 0.025 ; 0.075],}\end{array} \quad\right.$ for $i=1,3,6$ \\
\hline $20<t \leqslant 40$ & $\tau_{i}=$ According to Eqn. $(4.11)$ \\
\hline $40<t \leqslant 70$ & $\tau_{i}=\left\{\begin{array}{l}{[0.050 ; 0.025 ; 0.075],} \\
\text { According to Eqn. }(4.11)(\alpha=0.5), \quad \text { for } i=2,4,5\end{array}\right.$ \\
\hline $70<t \leqslant 200$ & $\tau_{i}=$ According to Eqn. $(4.11)$ \\
\hline \hline
\end{tabular}

states computed by the AUKF and PF. The PF seems to estimate the state with higher accuracy as compared to AUKF resulting in a faster convergence of formation-keeping attitude error and station-keeping attitude error.

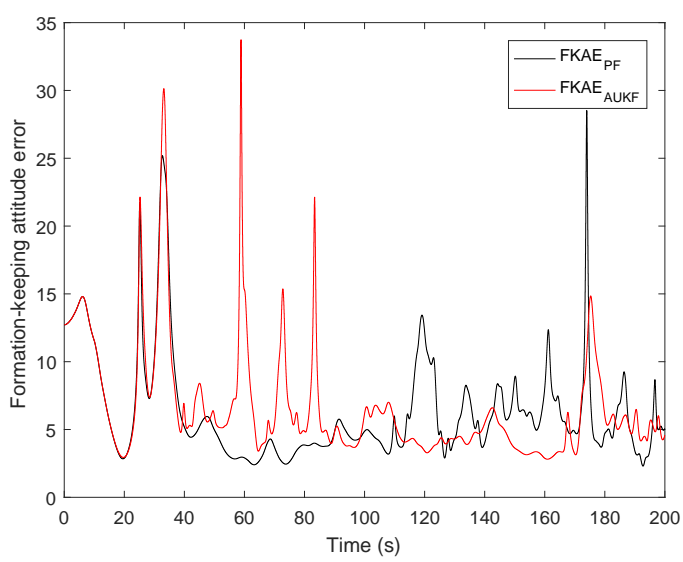

(a) Formation-Keeping Attitude Error

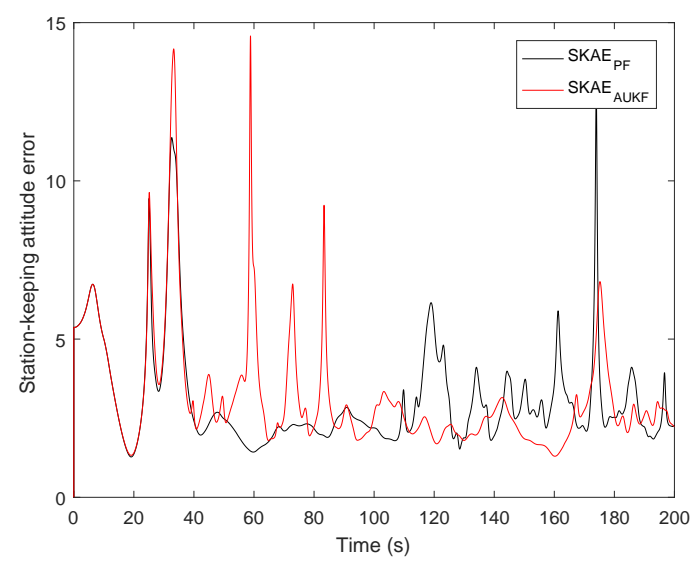

(b) Station-Keeping Attitude Error

Figure 4.6: No control command during formation stabilization. 


\subsection{Simulation Results and Discussion}

\subsubsection{Measurements noise}

In the following section, relatively large random measurement noise is introduced with the purpose of comparing the capacity of adaptive unscented Kalman filter and particle filter to estimate the attitude states and reduce the station-keeping and formation-keeping errors under heterogeneous measurement noise. Heterogeneous noise levels which are introduced in measurements may result from various interface sources include electromagnetic fields, temperature, pressure, contamination and high-energy radiation.

\section{Case I: Heterogeneous measurements}

In the following case, heterogeneous measurements are generated during the simulation time. High noise levels deteriorate the capacity to measure and estimate the attitude response of any dynamic system. Zero mean white Gaussian noise was used for both process noise and measurement noise; whereas, a random number generator was used in to draw samples at various noise levels. During the simulation, measurement sensitivity is altered at multiple intervals to include various noise fluctuations with the scope of comparing the capacity of AUKF and PF to estimate the spacecraft states and reduce the formationkeeping error and station-keeping error. The measurement noise are calculated as follows:

$$
\text { measurementNoise }=\text { Error }+\mathcal{N}(\mu, \sigma)
$$

where, error refers to typical measurement errors resulting from inaccuracies in measurement model, physical characteristics, or other environmental sources. $\mu$ is the mean value and, $\sigma$ is the uncertainty at different intervals during the simulation. These parameters are presented in Table (4.8). Figure 4.7(a) show the formation-keeping attitude error and Figure 4.7(b) show the station-keeping attitude error for spacecraft in formation flying in the presence heterogeneous measurements. It is interesting to note that between $t=0$ to $t=60$ when the noise level are relatively small, the formation-keeping error and stationkeeping error converge at the same rate for both AUKF and PF. This implies that both filters estimate the spacecraft states to the same accuracy. However, between $t=60$ to $t=100$ when the noise level increases, the particle filter estimates the spacecraft states at higher accuracy and is able to reduce the formation-keeping error and station-keeping 


\section{Chapter 4. Distributed Attitude Estimation and Control of Spacecraft}

Formation Flying

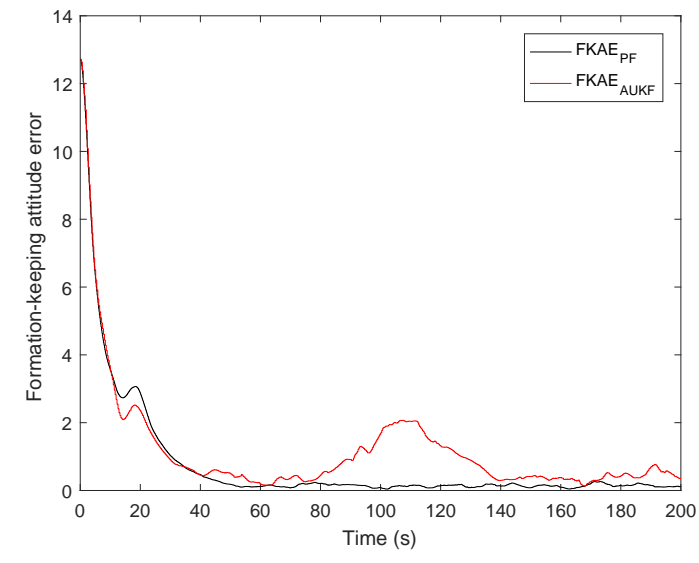

(a) Formation-Keeping Attitude Error

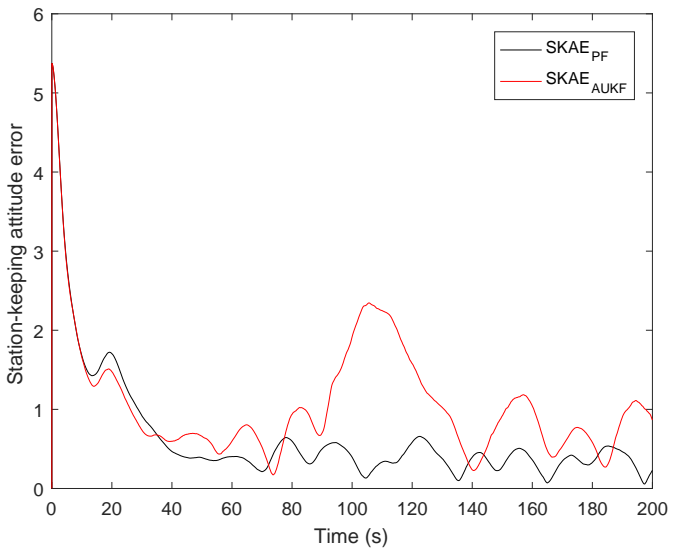

(b) Station-Keeping Attitude Error

Figure 4.7: Heterogeneous measurements.

errors to a larger extent as compared to AUKF.

Table 4.8: Heterogeneous measurements.

\begin{tabular}{|l|l|}
\hline \hline Time $[\mathrm{sec}]$ & Torque magnitude $\tau_{i}(i=1,2, \ldots, 6)$ \\
\hline \hline $0<t \leqslant 10$ & Error $=0.02 ; \mu=0 ; \sigma=1 \times 10^{-7}$ \\
\hline $10<t \leqslant 60$ & Error $=0.1 ; \mu=0 ; \sigma=1 \times 10^{-2}$ \\
\hline $60<t \leqslant 100$ & Error $=0.5 ; \mu=0 ; \sigma=1 \times 10^{-3}$ \\
\hline $100<t \leqslant 200$ & Error $=0.08 ; \mu=0 ; \sigma=1 \times 10^{-1}$ \\
\hline
\end{tabular}




\subsection{Conclusions}

\subsection{Conclusions}

The aim of this chapter was to investigate the estimation results of adaptive unscented Kalman filter and particle filtering technique and estimate the unmeasurable angular velocity and reference angular acceleration of spacecraft formation flying (SFF) while tracking a common time-varying reference attitude. The virtual leader exchanges only partial information with other spacecraft in the formation and only the neighboring spacecraft have access to information. Distributed filtering approach is proposed to obtain a reference angular acceleration estimates of the virtual leader and nonlinear filters were introduced to obtain an accurate estimation of unmeasurable angular velocity. The AUKF represent the state of the system using a Gaussian distribution while the particle filter represent the required posterior distribution by a set of random samples with associated weights and compute the estimates based on these samples. The samples are random particles of the hidden states and the weights are the probability masses estimated using the Bayesian recursion. The particle filter and adaptive unscented Kalman filter (AUKF) are compared in terms of the accuracy of estimating the unmeasurable angular velocity with the purpose of decreasing the station-keeping and formation-keeping attitude errors. The formation flying was comprised of six spacecraft including a virtual leader which exchange partial information with other spacecraft in the formation. Particle filter has a clear advantage over AUKF and provide better state estimates under fault cases. 
Chapter 5. Online Estimation and Control of Multi-Agent Systems

\section{CHAPTER 5}

\section{Online Estimation and Control of}

\section{Multi-Agent Systems}

In this chapter, a filter for online joint estimation is proposed based on Rao-Blackwellised principle where the particle filtering technique is coupled with unscented transform algorithm. The new filter is called marginalized unscented particle filter (MUPF). The proposed filter is applied in online joint estimation of an uncertain dynamic models which describe the evolution of a distributed multi-agent system in consensus tracking. A Chebyshev neural network $(\mathrm{CNN})$ is incorporated to describe the uncertain dynamic models of the multiagent system while nonlinear filtering policies are implemented to estimate the state of the multi-agent system and to train the internal parameters of the neural network as neatly as possible given a set of prior measurements. Much of the emphasis here is on accuracy of the joint estimation process, as well as the capacity to train CNN to determine the uncertain dynamics of the system. In this study, the AUKF and MUPF are compared under fault-free and fault-prone conditions at the multi-agent dynamics and control commands. These results are compared to the adaptive law which has been proposed for estimating the internal parameters of CNN. The chapter is organized as follows: Section 5.1 provide a brief introduction to the proposed marginalized unscented particle filter (MUPF) architecture, including the problem of estimating uncertain dynamic systems is outlined. Section 5.2.2 present the dynamic equation of a multi-agent system, the lumped error calculations, the control command and joint estimation algorithms. Section 5.3 provide the estimation results of uncertain dynamic of multi-agent system. The result compare between the adaptive unscented Kalman filter (AUKF), the marginalized unscented particle filter (MUPF), and the adaptive law under fault-free and fault-prone conditions. 


\subsection{Introduction}

\subsection{Introduction}

Sequential state and parameter estimation of dynamic systems is known in the literature as a joint estimation process. If parameter uncertainty is introduced in dynamic model, the joint estimation is simultaneously arises. The mathematical framework of estimation theory provide the tools required to approach the joint estimation problem from a Bayesian perspective. The basic idea is to characterize the posterior distribution of the state and parameters of a dynamic system at current time given the knowledge of prior distribution and evidence from new measurements. Since there is no optimal solution to the joint estimation process, suboptimal solutions are investigated.

The unscented Kalman filter offers an easy approach for sequential joint approximation of a Gaussian distributions [Julier \& Uhlmann 1997]. The filter provide an efficient derivative-free filtering algorithm for computing approximate solutions to discrete-time nonlinear filtering problems. The idea is to calculates the statistics of Gaussian random variables which undergone a nonlinear transformation through implementation of unscented transformation (UT) principles. The UT is a deterministic sampling technique that chooses a set of specific sample points around the mean i.e., sigma points. When the sigma points are propagated through the nonlinear transition and observation models, the mean and covariance of the state estimates can be accurately captured (for details see Chapter 2.4).

Another popular sequential filtering strategy is the particle filter which provide a framework for online joint estimation by combining Monte-Carlo sampling methods with Bayesian filter [Gordon \& Smith 1993]. The idea of particle filters is to represent the posterior distribution by a properly weighted support points, i.e., particles, which are sampled directly from the state-space. The particles are properly located, weighted, and propagated recursively according to the Bayesian rule. Particle filters rely on importance sampling principle which is aimed at sampling from a distribution in the region of "importance" in order to approximate the posterior distribution. Therefore, a good proposal distribution is essential to the efficiency of importance sampling and choosing an appropriate proposal distribution is a crucial step in the implementation of particle filters. For more details, the reader may refer to Chapter 2.8, or [Arulampalam et al. 2002], [Chen 2003], [Doucet \& Johansen 2008]. 
Chapter 5. Online Estimation and Control of Multi-Agent Systems

Extensive research has been placed on identifying techniques to improve the efficiency and reduce the variance of estimations in nonlinear filters. In this chapter, an idea to improve the joint estimation process is proposed where Rao-Blacwellization principle is used to couple the particle filter with unscented transform algorithm. The proposed filter is applied in online estimation of an uncertain dynamic models which describe the evolution of a distributed multi-agent system in consensus tracking. The purpose of the filter is to trains the internal parameters of the third-order Chebyshev neural network (CNN) as neatly as possible given a set of prior measurements.

The proposed idea to improve the efficiency of a particle filter is by using a RaoBlacwellization technique to evaluate some of the filtering parameters analytically instead pure sampling methods. The technique is known as Rao-Blacwellization particle filter (RBPF) [Casella \& Robert 1996], [Doucet et al. 2000], [Murphy \& Russell 2001]. The idea behind RBPF is that by conditioning a deterministic portion of the measurements, the posterior distribution can be approximated with a Gaussian distribution while the other measurements are approximated with a sampling techniques. The improvement is obtained by calculating a conditional expectation which involve integrating out the ancillary statistics. If the model admits partial analytical tractability, it is possible to combine the particle filter with optimal filters to obtain efficient high dimensional filters [Andrieu et al. 2001]. In this way, better estimates are obtained with less variance than could be obtained with pure Monte Carlo sampling [Särkkä 2013].

In this chapter, an addition to the toolbox of estimation algorithms is proposed such that the Rao-Blackwellised principal couples the particle filter with the unscented transform algorithm. The new filter is called marginalized unscented particle filter (MUPF). Here, the MUPF is used to estimate the state and parameters of an uncertain dynamic models of a multi-agent system. The proposed approach is entirely different than other approaches since the complex task of joint estimation is broken down into state estimation using the particle filter while the internal parameters of a third-order Chebyshev neural networks are estimated using unscented transform principles. Here, the third-order CNN describes the uncertain dynamic model of a multi-agent system and the weights of the network are updated at each iteration. 


\subsection{Introduction}

\subsubsection{Literature review}

A multi-agent system frequently distribute the task responsibilities among a number of individual agents, each operating with partial information of state estimates and response of other agents. In this environment, each agent operate in a decentralized architecture based on the information received. Generally, the dynamic behavior of a multi-agent system is derived from a theoretical or physical model, assuming the analytical form of the underlying physical laws are known from first principle. However, in many practical applications the description of a dynamic model is not exact and the physical laws governing the system are unknown or partially known. A broad range of techniques have been developed to deal with scenarios of unknown dynamic models and the theory become quite sophisticated. One of the proposed techniques have roots in neural networks (NN) which approximate any smooth function to an arbitrary accuracy. This makes NN suitable for estimating the uncertain nonlinear dynamics of a multi-agent system.

The principles of NN are based on a mapping between a set of input measurements arriving at an input layer and a set of output estimates at an output layer. The mapping between inputs to output layers is completed through a hidden layer. If the right connections from the input layer to a large enough set of hidden layer exist, a representation can always be found such that any mapping will be performed from input to output layer through these hidden units [Rumelhart et al. 1986]. In general, the first layer has input measurements which send information to a junction of a second layer of measurements, and then from the second junction to the third layer of output estimates. The junctions store parameters called "weights" that manipulate the measurements in the calculations [Bishop 1995]. In this work, a third-order Chebyshev neural networks (CNN) have been implemented as a universal approximation to learn the uncertain nonlinear functions in the agent dynamics (for details see Chapter 2.9). An-Min Zou, Krishna Dev Kumar, and Zeng-Guang Hou [Zou et al. 2011] have applied CNN to approximate an uncertain nonlinear functions in the problem of consensus tracking control for third-order multi-agent systems in the presence of uncertain dynamics and bounded external disturbance. In their work, the weights of the CNN were computed using the adaptive law. A similar work has been proposed in Arthur Kar Leaung Lin [Lin 2014], who studied multi-agent systems that orbit around asteroids. 
Chapter 5. Online Estimation and Control of Multi-Agent Systems

The contribution of this work is different in a sense that the joint estimation process of the multi-agent system is trained using sequential Bayesian approach. Joint estimation process for nonlinear dynamic systems is practically challenging and an open issue which arises when state and parameter uncertainty is introduced in the dynamic model. The basic idea is to characterize the posterior distribution of the state and parameters of the multi-agent system at current time given the knowledge of prior distribution and evidence from new measurements. In this case, particle methods are primarily used to construct a sequential distribution of the state and parameters by allowing particles to fully explore the posterior distributions. One of the major drawbacks of particle filtering is that sampling in high-dimensional state-spaces can be inefficient because of the large number of samples needed to represent the posterior distribution. To alleviate this issue, the Rao-Blackwell theorem was introduced to particle filtering [Doucet 1998], [de Freitas 2002].

Rao-Blackwell theory [Casella \& Robert 1996] is a marginalization technique that is used to reduce the variance of estimation. This marginalization technique is one of the ways for improving the efficiency and reducing the variance of estimation in particle filters [Doucet et al. 2000]. The implementation of Rao-Blackwell technique in particle filtering is known variously in the scientific literature as Rao-Blackwellized particle filter (RBPF) [Fong et al. 2002] [Särkkä et al. 2007], [Johansen et al. 2012], marginalized particle filter (MPF) [Schön et al. 2006], [Nordlund \& Gustafsson 2009] or mixtures Kalman filters [Chen \& Liu 2000]. The underlying principle of the technique is to increase the efficiency of the sampling method by reducing the size of the state-space model. This is done by marginalizing out some of the variables analytically by combining the particle filter with an optimal filter. The class of models where marginalization using Rao-Blackwell of a linear state components can be carried out usually depends on conditional linear Gaussian models. In this chapter, the linearity restriction of RBPF technique is partially relaxed by incorporating a sub-optimal filter. A similar idea has been proposed by Vihola [Vihola 2007], and in the textbook by Simo Särkkä (Chapter 7.5) [Särkkä 2013]; however, there is no documented literature to support the proposal. The Rao-Blackwell theorem and the mathematical derivations of RBPF are given in Appendix B. 


\subsection{Introduction}

\subsubsection{Objectives}

The objective of this research is to estimate the state and parameters of an uncertain dynamic model which describe the evolution of a distributed multi-agent system in consensus tracking. A nonlinear filtering technique is proposed such that the filter is capable of joint estimation in an online setting. The proposed filter reduces the dimensions of the state-space model and consequently reduce the variance of estimations by using the RaoBlackwellised principle to couple the particle filter technique with unscented transform algorithm. The main contributions of this research are as follows:

1. Estimation of uncertain dynamic models: Online estimation of an uncertain dynamic models using nonlinear filtering techniques and the approximation property of Chebyshev neural network (CNN). A third-order CNN is used as a universal approximation to learn in a sequential setting an uncertain nonlinear function which describe the agent dynamics. A nonlinear filter is developed and implemented to estimate the state of the multi-agent system and the internal parameters of CNN. An investigation is conducted to study a distributed multi-agent system in the presence of unknown system dynamics and external disturbance.

2. Propose a nonlinear filter for joint estimation: A nonlinear filtering technique is proposed for online joint estimation which couples the particle filtering technique with the unscented transform through the Rao-Blackwellised principle. The proposed filter is used simultaneously in state estimation of a multi-agent system in consensus tracking and parameter estimation of a third-order Chebyshev neural network. Much of the emphasis here is on accuracy of the joint estimation process, as well as the capacity to estimate the parameters of CNN and determine the uncertain dynamics of the multi-agent system. The proposed filter allow to simplify the high dimensional state-space model of the multi-agent system and jointly estimate the state and parameters in sequential setting.

3. Estimation of unbounded parameters: In contrast to existing parameter estimation approaches, the estimation algorithms presented herein are not bounded to a predefined set of values while the estimated uncertain dynamic function is properly 
Chapter 5. Online Estimation and Control of Multi-Agent Systems

updated sequentially as new measurements arrive. The unbounded weight estimation really goes down to a parameter estimation problem where the weights of CNN are treated as parameters and are updated sequentially as new measurements become available.

4. Evaluation of performance: Performance evaluation and comparison between the proposed nonlinear filter and other filtering techniques under fault-free and faultprone conditions. A comparison is conducted between the MUPF and AUKF under faults at the multi-agent dynamics and control commands. These results are compared to the adaptive law which has been proposed for estimating the internal parameters of CNN. The performance comparison in this research are illustrated with numerical simulations.

\subsection{System Model, Filter Model, and Control Law}

In this section, dynamic equations, kinematic equations, filter models, and control laws are constructed for a distributed multi-agent system in consensus tracking with uncertain dynamic model. Figure 5.1 shows the communication graph for the multi-agent system.

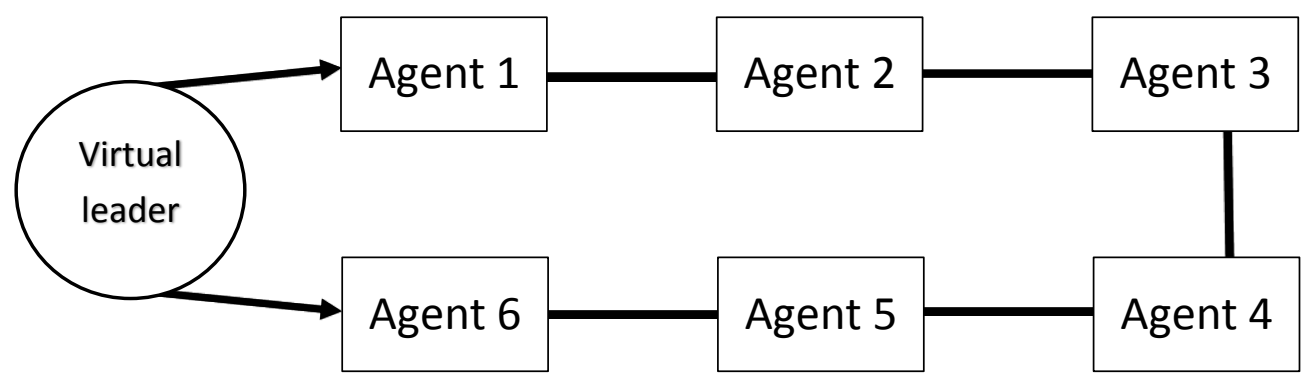

Figure 5.1: Communication graph for multi-agent system 


\subsection{System Model, Filter Model, and Control Law}

\subsubsection{Dynamic equations}

The term decentralized is often applied to systems comprising of multiple filters or controllers. Consider a class of nonlinear multi-agent systems with $n$ agents in which the $i^{\text {th }}$ agent is considered by the following second-order differential equation as

$$
\begin{gathered}
\dot{\mathrm{x}}_{i}=\mathrm{v}_{i} \\
\dot{\mathrm{v}}_{i}=f_{i}\left(\mathrm{x}_{i}, \mathrm{v}_{i}\right)+\mathrm{g}_{i}\left(\mathrm{x}_{i}\right) u_{i}+\vartheta_{i}, \quad i=1,2, \cdots, n
\end{gathered}
$$

where $\mathrm{x}_{i} \in R^{m}$ and $\mathrm{v}_{i} \in R^{m}$ are the state vectors of the $i^{\text {th }}$ agent, $f_{i}\left(\mathrm{x}_{i}, \mathrm{v}_{i}\right) \in R^{m}$ is a continuous unknown dynamic function, $\mathrm{g}_{i}\left(\mathrm{x}_{i}\right) \in R^{m \times m}$ is a nonsingular control gain matrix, $u_{i} \in R^{m}$ is the control input of the $i^{\text {th }}$ agent, and $\vartheta_{i} \in R^{m}$ is the external disturbance.

\subsubsection{Joint estimation model}

The basic idea of joint estimation is to characterize the posterior distribution of the state and parameters of the dynamic model at current time given the knowledge of prior distribution and evidence from new measurements. To be more specific, consider the problem of estimating both the hidden state $\mathrm{x}_{k}$ and parameter $\theta_{k}$ of a discrete multi-agent system

$$
\begin{aligned}
\mathrm{z}_{k+1} & =f\left(\mathrm{x}_{k}, \theta_{k}, u_{k}, w_{k}\right) \\
\mathrm{y}_{k} & =h\left(\mathrm{x}_{k}, \theta_{k}, v_{k}\right)
\end{aligned}
$$

where $\mathrm{z}_{k+1}$ denotes the joint estimation vector which contains the parameter of interest and the state vector. The parameter of interest, $\theta_{k} \in R^{m \times N_{1}}$, denotes the neural network weights ${ }^{1}$, and $\mathrm{x}_{k}$ is an $n$-dimensional state vector with initial state $\mathrm{x}_{0}$. The function $f: \Re^{n_{\mathrm{x}}} \times \Re^{n_{v}} \rightarrow \Re^{n_{\mathrm{x}}}$, denotes the state transition models in Equations (5.1)-(5.2), and the function $h: \Re^{n_{\times}} \times \Re^{w_{n}} \rightarrow \Re^{n_{y}}$ is a nonlinear mapping of the measurements. $u_{k}$ denotes a deterministic control input, $w_{k}$ and $v_{k}$ are the transition and measurement noise, respectively which are assumed to be uncorrelated with the network weights and initial conditions. The evolution of the network weights corresponds to a Markov process with initial probability $p\left(\theta_{k=0}\right)$ and transition probability $p\left(\theta_{k} \mid \theta_{k-1}\right)$.

\footnotetext{
${ }^{1}$ The evolution of the network weights are assumed to depend on the previous weight $\theta_{k-1}$ and additional stochastic parameter $d_{k-1}$.
} 


\section{Chapter 5. Online Estimation and Control of Multi-Agent Systems}

\subsubsection{Filter model}

Filtering techniques are investigated for estimating the weights of a neural network which approximate an unknown nonlinear function. The filter model for estimating the state a multi-agent system is given by

$$
\hat{\mathrm{z}}_{k+1}=f\left(\hat{\theta}_{k}, \hat{\mathrm{x}}_{k}, \tau_{k}\right)+w_{k}
$$

where $w_{k}$ is the process noise, $\tau_{k}$ is the control torque, $\hat{\mathrm{x}}_{k}$ is the estimated state vector, $\hat{\theta}_{k}=\left[W_{m \times 1}^{*}, W_{m \times 2}^{*}, \cdots, W_{m \times N_{1}}^{*}\right]^{T}$ is the estimated neural network weight, and $f(\cdot)$ is propagated through the forth-order Runge-Kutta method given by Equation (3.7) with parameters defined by Equation (3.8) and with function $G(\hat{\mathrm{x}})$ given as follows

$$
G(\hat{\mathrm{x}})=\left[\begin{array}{c}
\dot{\mathrm{x}}_{i}=\mathrm{v}_{i} \\
\dot{\mathrm{v}}_{i}=f_{i}^{c n n}\left(\mathrm{x}_{i}, \mathrm{v}_{i}\right)+\mathrm{g}_{i}\left(\mathrm{x}_{i}\right) u_{i}, \quad i=1,2, \cdots, n
\end{array}\right]
$$

Estimating the neural network weights is a parameter estimation process where the optimal weight $W_{i}^{*}$ is estimated in online setting during the simulation. The unknown function $f_{i}^{c n n}\left(\mathrm{x}_{i}, \mathrm{v}_{i}\right)$ in Equation (5.5) is approximated by a property of Chebyshev neural network

$$
f_{i}^{c n n}\left(\mathrm{x}_{i}, \mathrm{v}_{i}\right)=W_{i}^{*} \xi_{i}\left(\mathrm{x}_{i}, \mathrm{v}_{i}\right)+\varepsilon_{i}
$$

where $W_{i}^{*} \in R^{m \times N_{1}}$ is the optimal weight with $N_{1}=2 m N_{2}+1$ and $N_{2}$ is the order of the Chebyshev polynomials, $\varepsilon_{i} \in R^{m}$ is the CNN approximation error, and $\xi_{i}\left(X_{i}\right) \in R^{N_{1}}$ with $X_{i}=\left(\mathrm{x}_{i}^{T}, \mathrm{v}_{i}^{T}\right)^{T}$ denotes the Chebyshev polynomial basis function. The polynomial basis function $\xi_{i}\left(X_{i}\right)(i=1,2, \cdots, n)$ is obtained by the Chebyshev polynomial

$$
\xi_{i}\left(X_{i}\right)=\left(1, T_{1}\left(X_{i, 1}\right), \cdots, T_{N_{2}}\left(X_{i, 1}\right), \cdots, T_{1}\left(X_{i, 2 m}\right), \cdots, T_{N_{2}}\left(X_{i, 2 m}\right)\right)^{T}
$$

where $T_{i}\left(X_{i, j}\right)\left(i=1,2, \cdots, N_{2}, j=1,2, \cdots, 2 m\right)$ represents the Chebyshev polynomials which are obtained using the recursive formula defined by Equation (2.43). 


\subsection{System Model, Filter Model, and Control Law}

\subsubsection{Marginalized unscented particle filter}

The idea of MUPF is to partition the dynamic state space into two subspaces $\mathrm{z}_{0: k}=$ $\left\{\theta_{0: k}, \mathrm{x}_{0: k}\right\}$, such that the distribution $p\left(\theta_{0: k}, \mathrm{x}_{0: k} \mid \mathrm{y}_{1: k}\right)$ can be updated analytically and efficiently. The parameter of interest, $\theta_{k} \in R^{m \times N_{1}}$ is the optimal neural network weight with $N_{1}=2 m N_{2}+1$ where $N_{2}$ is the order of the Chebyshev polynomial, and $\mathrm{x}_{k}$ is the $n$-dimensional state vector with initial state $\mathrm{x}_{0}$. Since the state $\mathrm{x}_{k}$ conditioned on $\theta_{k}$ is reduced to nonlinear Gaussian state-space system, then the estimation is performed analytically using the adaptive unscented Kalman filter ${ }^{2}$. The variance of estimation is reduced by marginalizing out the parameter $\mathrm{x}_{0: k}$ such that the full posterior distribution at time step $k$ can be factorized as follows

$$
p\left(\theta_{0: k}, \mathrm{x}_{0: k} \mid \mathrm{y}_{1: k}\right)=p\left(\mathrm{x}_{0: k} \mid \mathrm{y}_{1: k}, \theta_{0: k}\right) p\left(\theta_{0: k} \mid \mathrm{y}_{1: k}\right)
$$

where adaptive unscented Kalman filter is used to evaluate the term $p\left(\mathrm{x}_{0: k} \mid \mathrm{y}_{1: k}, \theta_{0: k}\right)$ since it is nonlinear and Gaussian, whereas the second term, $p\left(\theta_{0: k} \mid \mathrm{y}_{1: k}\right)$ is evaluated using particle filter. From the second term the following recursion is obtained [Särkkä 2013]

$$
\begin{aligned}
p\left(\theta_{0: k} \mid \mathrm{y}_{1: k}\right) & \propto p\left(\mathrm{y}_{k} \mid \theta_{0: k}, \mathrm{y}_{1: k-1}\right) p\left(\theta_{0: k} \mid \mathrm{y}_{1: k-1}\right) \\
& =p\left(\mathrm{y}_{k} \mid \theta_{0: k}, \mathrm{y}_{1: k-1}\right) p\left(\theta_{k} \mid \theta_{0: k-1}, \mathrm{y}_{1: k-1}\right) p\left(\theta_{0: k-1} \mid \mathrm{y}_{1: k-1}\right) \\
& =p\left(\mathrm{y}_{k} \mid \theta_{0: k}, \mathrm{y}_{1: k-1}\right) p\left(\theta_{k} \mid \theta_{k-1}\right) p\left(\theta_{0: k-1} \mid \mathrm{y}_{1: k-1}\right)
\end{aligned}
$$

The first term is evaluated using AUKF with fixed $\theta_{0: k}$ over the measurement model, the second term is the transition model, and the third term is the posterior from the previous time step. Therefore, sampling the parameter $\theta_{k}$ will generally require many fewer particles than standard particle filter which would sample both $\mathrm{x}_{k}$ and $\theta_{k}$. The $\theta_{k}$ samples are updated as in standard particle filter and the $\mathrm{x}_{k}$ distribution are updated using the AUKF, conditioned on the parameter $\theta_{k}$. Next, the algorithm for marginalized unscented particle filter is described.

\footnotetext{
${ }^{2}$ If the state $\mathrm{x}_{1: k}$, conditioned on $\theta_{1: k}$ could be reduced to a linear Gaussian state-space system, then the estimation can be performed using Kalman filter. This method is known as Rao-Blackwellized Particle Filter (RBPF) [Doucet 1998], [de Freitas 2002].
} 
Chapter 5. Online Estimation and Control of Multi-Agent Systems

MARGINALIZED UNSCENTED PARTICLE FILTER (MUPF)

1. Prediction step using AUKF (see AUKF algorithm in Chapter2.4)

$$
\begin{aligned}
\overline{\mathrm{x}}_{k} & =\sum_{i=0}^{2 n} W_{i}^{(m)} \hat{\mathcal{X}}_{k}^{(i)}\left(\theta_{k-1}^{(i)}\right) \\
P_{x x} & =\sum_{i=0}^{2 n} W_{i}^{(c)}\left[\hat{\mathcal{X}}_{k}^{(i)}\left(\theta_{k-1}^{(i)}\right)-\overline{\mathrm{x}}_{k}\right]\left[\hat{\mathcal{X}}_{k}^{(i)}\left(\theta_{k-1}^{(i)}\right)-\overline{\mathrm{x}}_{k}\right]^{T}+Q
\end{aligned}
$$

2. For $k=1, \ldots, T$

(a) Draw samples from the importance distribution

$$
\theta_{k}^{(i)} \sim q\left(\theta_{k}^{(i)} \mid \theta_{0: k-1}^{(i)}, \mathrm{y}_{1: k}\right), \quad i=1,2, \ldots, N_{p}
$$

(b) Calculate the updated importance weights

$$
w_{k}^{(i)}=w_{k-1}^{(i)} \frac{p\left(\mathrm{y}_{k} \mid \theta_{k}^{(i)}, \mathrm{y}_{1: k-1}\right) p\left(\theta_{k}^{(i)} \mid \theta_{k-1}^{(i)}\right)}{q\left(\theta_{k}^{(i)} \mid \theta_{0: k-1}^{(i)}, \mathrm{y}_{1: k}\right)}, \quad i=1,2, \ldots, N_{p}
$$

where the likelihood function is computed from AUKF and is conditioned on $\theta_{k}^{(i)}$ such that $p\left(\mathrm{y}_{k} \mid \theta_{k}^{(i)}, \mathrm{y}_{1: k-1}\right)=\sum_{i=0}^{2 n} W_{i}^{(m)} \hat{\mathcal{Y}}_{k}^{(i)}\left(\theta_{k-1}^{(i)}\right)$ where $\hat{\mathcal{Y}}_{k}^{(i)}\left(\theta_{k-1}^{(i)}\right)=h_{k}\left(\mathcal{X}_{k-1}^{(i)}, \theta_{k-1}^{(i)}\right)$.

3. Normalize the importance weight such that $\sum_{i}^{N_{p}} \tilde{w}_{k}^{(i)}=1$

$$
\tilde{w}_{k}^{(i)}=\frac{w_{k}^{(i)}}{\sum_{j=1}^{N_{p}} w_{k}^{(j)}}
$$

4. AUKF gain calculation and update (see AUKF algorithm in Chapter2.4)

$$
\begin{aligned}
K_{k} & =P_{x y} P_{y y}^{-1} \\
\hat{\mathrm{x}}_{k} & =\overline{\mathrm{x}}_{k}+K_{k}\left(\mathrm{y}_{k}-\overline{\mathrm{y}}_{k}\right) \\
P_{k}^{+} & =P_{x x}-K_{k} P_{y y} K_{k}^{T}
\end{aligned}
$$

5. Compute effective sample size and resample

$$
\hat{N}_{e f f}=\frac{1}{\sum_{i=1}^{N_{p}}\left(w_{k}^{i}\right)^{2}}
$$

If $\hat{N}_{e f f}$ is small perform resampling. 


\subsection{System Model, Filter Model, and Control Law}

\section{Approximate the posterior distribution}

$$
p\left(\theta_{0: k}, \mathrm{x}_{0: k} \mid \mathrm{y}_{1: k}\right) \approx \sum_{i=1}^{N_{p}} \tilde{w}_{k}^{(i)} \delta\left(\theta_{0: k}-\theta_{0: k}^{(i)}\right) \mathcal{N}\left(\mathrm{x}_{k} \mid \hat{\mathrm{x}}_{k}, P_{k}^{+}\right)
$$

\subsubsection{Control law}

The control law for the $i^{\text {th }}$ agent in the multi-agent system is [Zou et al. 2011]

$$
u_{i}=\mathrm{g}_{i}^{-1}\left(-W_{i}^{*} \xi_{i}-K_{1 i} s_{i}-K_{2 i} s_{i}-\psi_{i}\right), \quad i=1,2, \cdots, n
$$

where $K_{1 i}$ and $K_{2 i}$ are positive definite, diagonal, and constant matrices. The robust control term $\psi_{i} \in R^{m}(i=1,2, \cdots, n)$ is used to counteract the external disturbance and neural network approximation error,

$$
\psi_{i j}=\kappa_{i} \tanh \left(\frac{m k_{u} \kappa_{i} s_{i j}}{\varepsilon}\right), \quad k_{u}=0.2785, \quad j=1,2, \cdots, m
$$

where $\kappa_{i}$ and $\varepsilon$ are positive constants. The terminal sliding manifold $s_{i} \in R^{m}(i=$ $1,2, \ldots, n)$ is expressed as

$$
s_{i}=\alpha_{\mathrm{v} i}+\sigma_{1} \alpha_{\mathrm{x} i}+\sigma_{2} \beta_{i}
$$

where

$$
\begin{gathered}
s_{i}=\left(s_{1}^{T}, s_{2}^{T}, \ldots, s_{n}^{T}\right)^{T} \in R^{m n} \quad \beta_{i}=\left(\beta_{1}^{T}, \beta_{2}^{T}, \ldots, \beta_{n}^{T}\right)^{T} \in R^{m n} \\
\sigma_{1}=\operatorname{diag}\left\{\sigma_{11} I_{m}, \sigma_{12} I_{m}, \ldots, \sigma_{1 n} I_{m}\right\} \in R^{(m n) \times(m n)} \\
\sigma_{2}=\operatorname{diag}\left\{\sigma_{21} I_{m}, \sigma_{22} I_{m}, \ldots, \sigma_{2 n} I_{m}\right\} \in R^{(m n) \times(m n)}
\end{gathered}
$$

and $\alpha_{\mathrm{x} i} \in R^{m}$ and $\alpha_{\mathrm{v} i} \in R^{m}(i=1,2, \cdots, n)$ for the $i^{t h}$ agent are the lumped state error measures which are defined as the sum of the absolute and relative state errors and depends only on the information of the neighbor agents of the $i^{\text {th }}$ agent. The lumped state errors are defined as

$$
\begin{aligned}
& \alpha_{\mathrm{x} i}=\sum_{j=1}^{n} a_{i j} r_{\mathrm{x} i j}+b_{i} e_{\mathrm{x} i} \\
& \alpha_{\mathrm{v} i}=\sum_{j=1}^{n} a_{i j} r_{\mathrm{v} i j}+b_{i} e_{\mathrm{v} i}
\end{aligned}
$$




\section{Chapter 5. Online Estimation and Control of Multi-Agent Systems}

where $\alpha_{\mathrm{x}}=\left(\alpha_{\mathrm{x} 1}^{T}, \alpha_{\mathrm{x} 2}^{T}, \cdots, \alpha_{\mathrm{x} n}^{T}\right)^{T} \in R^{m n}$ and $\alpha_{\mathrm{v}}=\left(\alpha_{\mathrm{v} 1}^{T}, \alpha_{\mathrm{v} 2}^{T}, \cdots, \alpha_{\mathrm{v} n}^{T}\right)^{T} \in R^{m n}$, $a_{i j}$ is the element of the weighted adjacency matrix $^{3} A$, and the relative and absolute state errors are defined as follows:

Relative Error: The relative error is the state error of an individual agent with respect to another agent in the multi-agent system. The relative state error between the $i^{\text {th }}$ agent $(i=1,2, \cdots, n)$ and the $j^{\text {th }}$ agent $(j=1,2, \cdots, n)$ are defined by

$$
\begin{aligned}
& r_{\mathrm{x} i j}=\mathrm{x}_{i}-\mathrm{x}_{j} \\
& r_{\mathrm{v} i j}=\mathrm{v}_{i}-\mathrm{v}_{j}
\end{aligned}
$$

Absolute Error: The absolute error is the state error of an individual agent with respect to the desired trajectory. The absolute errors of the $i^{\text {th }}$ agent $(i=1,2, \cdots, n)$ are defined by

$$
\begin{aligned}
& e_{\mathrm{x} i}=\mathrm{x}_{i}-\mathrm{x}_{d} \\
& e_{\mathrm{v} i}=\mathrm{v}_{i}-\dot{\mathrm{x}}_{d}
\end{aligned}
$$

where $e_{\mathrm{x}}=\left(e_{\mathrm{x} 1}^{T}, e_{\mathrm{x} 2}^{T}, \cdots, e_{\mathrm{x} n}^{T}\right)^{T} \in R^{m n}$ and $e_{\mathrm{v}}=\left(e_{\mathrm{v} 1}^{T}, e_{\mathrm{v} 2}^{T}, \cdots, e_{\mathrm{v} n}^{T}\right)^{T} \in R^{m n}$. The common desired trajectory (i.e. $\mathrm{x}_{d}$ and $\dot{\mathrm{x}}_{d}$ ) is available to only a subset of agents and each agent in the group has access to information of its neighbor agents only as expressed by the adjacency matrices $A$ and $B$ where $B=\operatorname{diag}\left\{a_{10}, a_{20}, \cdots, a_{i 0}\right\}$, and $a_{i 0}>0(i=1,2, \cdots, n)$ is a constant if the $i$ th spacecraft has access to the leader, otherwise $a_{i 0}=0$.

\footnotetext{
${ }^{3}$ The weighted adjacency matrix $A$ denotes the communication quality between the $i^{\text {th }}$ agent and the $j^{t h}$ agent and the weighted adjacency element $a_{i j}$ is bounded, such that, $a_{i i}=0$ and $0<a_{i j}<1$.
} 


\subsection{Simulation Results and Discussion}

\subsection{Simulation Results and Discussion}

In the following section, numerical simulations are presented for online consensus tracking of a distributed multi-agent system under fault-free and fault-prone scenarios. Here, the time-varying reference trajectory of a multi-agent system is provided by a virtual leader where the desired trajectory is available to a subset of the agents and each agent in the group has restricted access to information of its neighbor agents only. The purpose is to estimate the uncertain dynamic function in an online setting where the dynamic function describes the evolution of a distributed multi-agent system. The uncertain dynamic function is modeled as a third-order Chebyshev neural network (CNN) and three filtering algorithms are proposed to train the internal weights of CNN, namely, adaptive law, adaptive unscented Kalman filter and marginalized unscented particle filter. An emphasis is placed on training the weights of a third-order CNN while constraining the work to sequential training and prediction. Sampling techniques in state-space setting show how a CNN may be trained in environments where measurements arrive one at a time.

The communication quality between the virtual leader and each agent is expressed with a symmetric weighted adjacency matrix. For simulation purposes, the weighted adjacency matrices are given as follows

$$
\begin{gathered}
A=\left[\begin{array}{llllll}
0.0 & 0.7 & 0.0 & 0.0 & 0.0 & 0.0 \\
0.7 & 0.0 & 0.6 & 0.0 & 0.0 & 0.0 \\
0.0 & 0.6 & 0.0 & 0.7 & 0.0 & 0.0 \\
0.0 & 0.0 & 0.7 & 0.0 & 0.8 & 0.0 \\
0.0 & 0.0 & 0.0 & 0.8 & 0.0 & 0.9 \\
0.0 & 0.0 & 0.0 & 0.0 & 0.9 & 0.0
\end{array}\right] \\
B=\operatorname{diag}\left[\begin{array}{llllll}
0.8, & 0.0, & 0.0, & 0.0, & 0.0, & 0.9
\end{array}\right]
\end{gathered}
$$

The desired trajectory is given by $\mathrm{x}_{0}=0.5(\cos (t), \sin (t))^{T}$, and the initial weight matrices of the CNN are given as follows: $\mathrm{CNN}_{A U K F}=0_{2 \times 13}, \mathrm{CNN}_{M U P F}=0_{2 \times 13}, \mathrm{CNN}_{A D T L}=$ $0_{2 \times 13}$, for $i=1,2, \ldots, 6$. The performance of the proposed filters are presented with an absolute error matrix and a relative error matric. The absolute error matric (AER) is 


\section{Chapter 5. Online Estimation and Control of Multi-Agent Systems}

defined as

$$
A E M=\sqrt{\sum_{i=1}^{n}\left\|e_{\mathrm{x} i}\right\|^{2}}
$$

and the relative error matric (REM) is defined as

$$
R E M=\sqrt{\sum_{i=1}^{n-1} \sum_{j=i+1}^{n}\left\|r_{\mathrm{x} i j}\right\|^{2}}
$$

The estimation techniques considered herein are the adaptive law, adaptive unscented Kalman filter and marginalized unscented particle filter (MUPF).

Adaptive unscented Kalman filter: The composite scaling parameter ${ }^{4}$ is $\lambda=$ $\alpha_{A U K F}^{2} \times(n+\kappa)-n$, where $\alpha_{A U K F}=0.1, \beta_{A U K F}=2, \kappa=0$, and $n$ is the dimension of the augmented state. The filter weight are calculated according to $\mathrm{W}_{0}^{(m)}=\lambda /(n+\lambda)$, $\mathrm{W}_{0}^{(c)}=\lambda /(n+\lambda)+\left(1-\alpha_{A U K F}^{2}+\beta_{A U K F}\right)$, and $\mathrm{W}_{i}^{(m)}=\mathrm{W}_{i}^{(c)}=1 / 2(n+\lambda)$, for $i=1,2, \ldots, n$ where $\mathrm{W}_{i}^{(m)}$ and $\mathrm{W}_{i}^{(c)}$ are the weight associated with the mean and covariance matrices accordingly.

Adaptive law: The adaptive law has been proposed by An-Min Zou, Krishna Dev Kumar and Zeng-Guang Hou [Zou et al. 2011]. The adaptive law for $W_{i}(i=1,2, \cdots, n)$ is

$$
\dot{W}_{i}=\delta_{i} s_{i} \xi_{i}^{T}
$$

where $s_{i}$ is the terminal sliding manifold given by Equation (5.19), $\xi_{i}$ is the Chebyshev basis function given by Equation (5.7), and $\delta_{i}$ is a positive constant.

Marginalized unscented particle filter: The number of particles $N_{p}=100$, and the resampling threshold for Effective Sample Size (ESS), $\beta_{M U P F}=0.8$. For simplicity, the resampling algorithm selected is the multinomial resampling. The proposal distribution was selected from a known distribution such that $q\left(\theta_{k}^{(i)} \mid \theta_{0: k-1}^{(i)}, \mathrm{y}_{1: k}\right)=p\left(\theta_{k}^{(i)} \mid \theta_{k-1}^{(i)}, \mathrm{y}_{1: k}\right)$.

\footnotetext{
${ }^{4}$ The composite scaling parameter $\lambda$ is a function of three variables: (1) $\alpha_{A U K F}$ which determines the spread of the sigma points around the mean $\overline{\mathrm{x}},(2) \beta_{A U K F}$ which incorporate prior knowledge of the distribution of the state $\mathrm{x}$, and $(3) \kappa$ which is a secondary scaling parameter usually set to 0 .
} 


\subsection{Simulation Results and Discussion}

The parameters of the adaptive unscented Kalman filter which were implemented in the marginalized unscented particle filter were described in previous paragraph.

\subsubsection{Fault-Free multi-agent system}

In this section, the uncertain dynamic model of a distributed multi-agent system is estimated under fault-free scenario. The multi-agent system consists of a virtual leader and six follower agents, where the uncertain nonlinear function $f_{i}\left(\mathrm{x}_{i}, \mathrm{v}_{i}\right)$ is given by

$$
f_{i}\left(\mathrm{x}_{i}, \mathrm{v}_{i}\right)=\left(\begin{array}{c}
4 \mathrm{x}_{i 2} \sin \left(\frac{\pi}{4}+\frac{h_{1 i} \mathrm{v}_{i 1}}{2}\right) \\
4 \mathrm{x}_{i 1} \cos \left(\frac{h_{2 i} \mathrm{v}_{i 2}}{2}\right)
\end{array}\right)
$$

where the parameters $h_{1 i}$ and $h_{2 i}$ are given in Table 5.1, and $\mathrm{x}_{i}=\left(\mathrm{x}_{i 1}, \mathrm{x}_{i 2}\right)^{T}, \mathrm{v}_{i}=\left(\mathrm{v}_{i 1}, \mathrm{v}_{i 2}\right)^{T}$ are given in Table 5.2.

Table 5.1: Parameters of agent's dynamics

\begin{tabular}{|l|l|l|l|l|l|l|}
\hline \hline$i$ & 1 & 2 & 3 & 4 & 5 & 6 \\
\hline \hline$h_{1 i}$ & 0.6 & -0.2 & 0.4 & -0.3 & 0.5 & 0.5 \\
\hline$h_{2 i}$ & 0.3 & 0.4 & -0.4 & -0.7 & -0.2 & -0.5 \\
\hline \hline
\end{tabular}

The external disturbances $\vartheta_{i}(i=1,2, \ldots, 6)$ in the agent's dynamic model and the control gain matrix $\mathrm{g}_{i}$ is given by

$$
\vartheta_{i}=\left(\begin{array}{c}
0.1 \sin \left(\frac{i t}{2}\right) \\
0.1 \cos \left(\frac{i t}{2}\right)
\end{array}\right), \quad \mathrm{g}_{i}=I_{2}
$$

Next, simulation results are presented in Figures 5.2-5.6 for the adaptive unscented Kalman filter, marginalized unscented particle filter, and the adaptive law under fault-free scenario. Figure 5.2 shows the estimation results of the uncertain dynamic model $\hat{f}_{i}=\left(\hat{f}_{i 1}, \hat{f}_{i 2}\right)^{T}(i=$ $1,2, \ldots, 6)$. Figure 5.3 present the agent's state estimates $\hat{\mathrm{x}}_{i}=\left(\hat{\mathrm{x}}_{i 1}, \hat{\mathrm{x}}_{i 2}\right)^{T}(i=1,2, \ldots, 6)$; where, Figure 5.3 (a)-(b), are the state estimates using the adaptive law, Figure 5.3 (c)-(d), are the state estimates using the adaptive unscented Kalman filter, and Figure 5.3 (e)-(f), are the state estimates using the marginalized unscented particle filter. Figure 5.4 present 


\section{Chapter 5. Online Estimation and Control of Multi-Agent Systems}

Table 5.2: Initial conditions.

\begin{tabular}{|l|l|c|}
\hline \hline$i$ & $\mathrm{x}_{i}$ & $v_{i}$ \\
\hline \hline 1 & $\mathrm{x}_{1}(0)=[1,0.5]^{T}$ & $v_{1}(0)=[0,0]^{T}$ \\
\hline 2 & $\mathrm{x}_{2}(0)=[1.8,-0.2]^{T}$ & $v_{2}(0)=[0,0]^{T}$ \\
\hline 3 & $\mathrm{x}_{3}(0)=[0.2,-1.2]^{T}$ & $v_{3}(0)=[0,0]^{T}$ \\
\hline 4 & $\mathrm{x}_{4}(0)=[-0.1,1.2]^{T}$ & $v_{4}(0)=[0,0]^{T}$ \\
\hline 5 & $\mathrm{x}_{5}(0)=[-0.6,1.8]^{T}$ & $v_{5}(0)=[0,0]^{T}$ \\
\hline 6 & $\mathrm{x}_{6}(0)=[1,-1.7]^{T}$ & $v_{6}(0)=[0,0]^{T}$ \\
\hline
\end{tabular}

the agent's tracking error under fault-free scenario $e_{i}=\left(e_{i 1}, e_{i 2}\right)^{T}(i=1,2, \ldots, 6)$; where, Figure 5.4 (a)-(b), are the tracking errors using the adaptive law, Figure $5.4(\mathrm{c})$-(d), are the tracking errors using the adaptive unscented Kalman filter, and Figure 5.4 (e)-(f), are the tracking errors using the marginalized unscented particle filter. Figure 5.5 shows the control laws $u_{i}=\left(u_{i 1}, u_{i 2}\right)^{T}(i=1,2, \cdots, 6)$. Figure 5.6 (a) is the absolute error metric (AEM), and Figure 5.6 (b) is the relative error metric (REM). Finally, the weights of the third-order Chebyshev neural network are presented in Appendix C.2. 


\subsection{Simulation Results and Discussion}
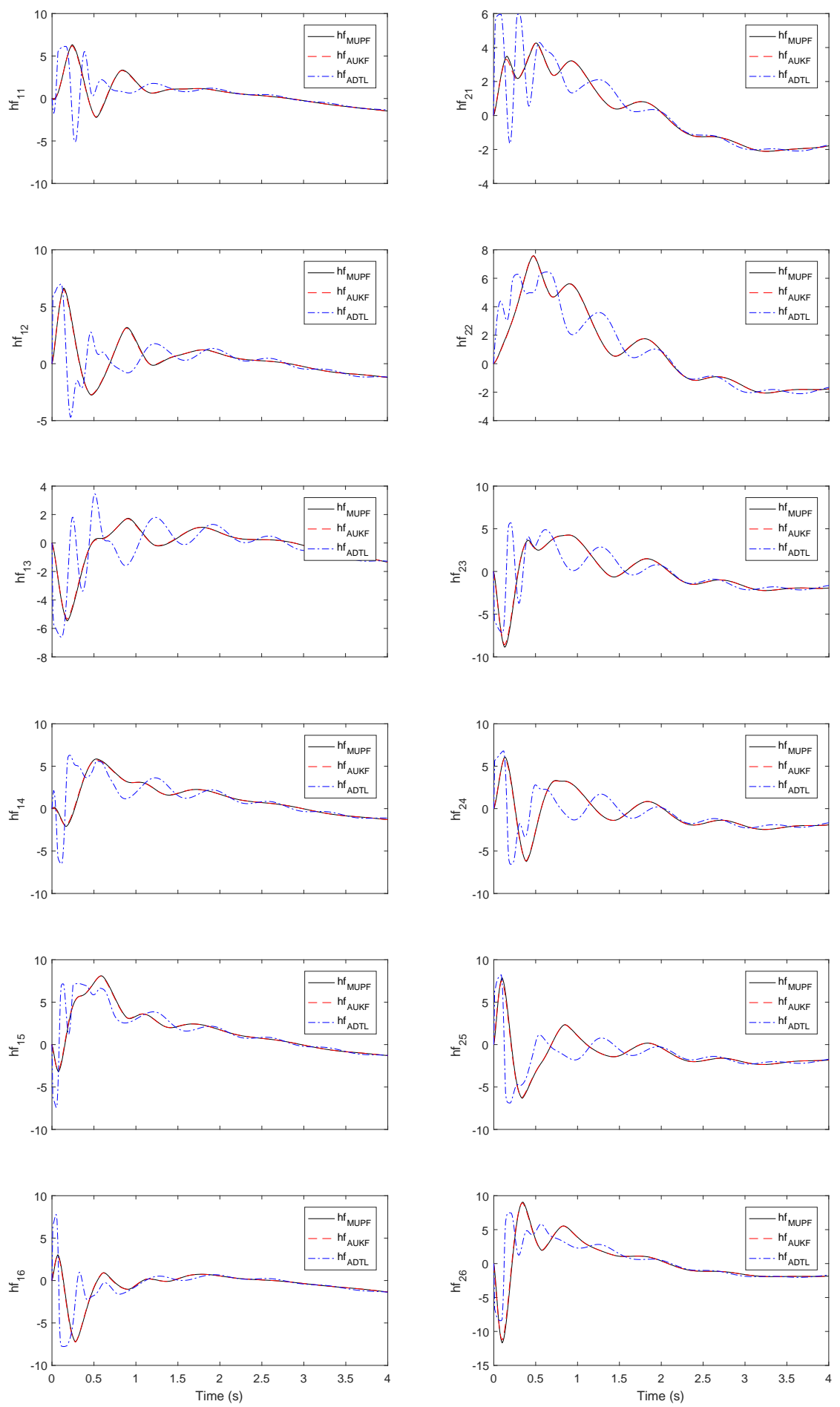

Figure 5.2: Estimation of uncertain dynamic model under fault-free scenario $\hat{f}_{i}=$ $\left(\hat{f}_{i 1}, \hat{f}_{i 2}\right)^{T}(i=1,2, \ldots, 6)$. 


\section{Chapter 5. Online Estimation and Control of Multi-Agent Systems}

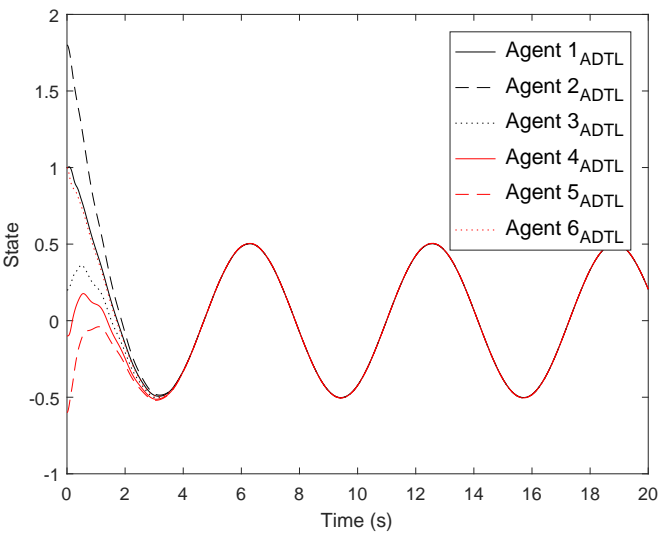

(a) $\hat{\mathrm{x}}_{i 1_{A D T L}}$

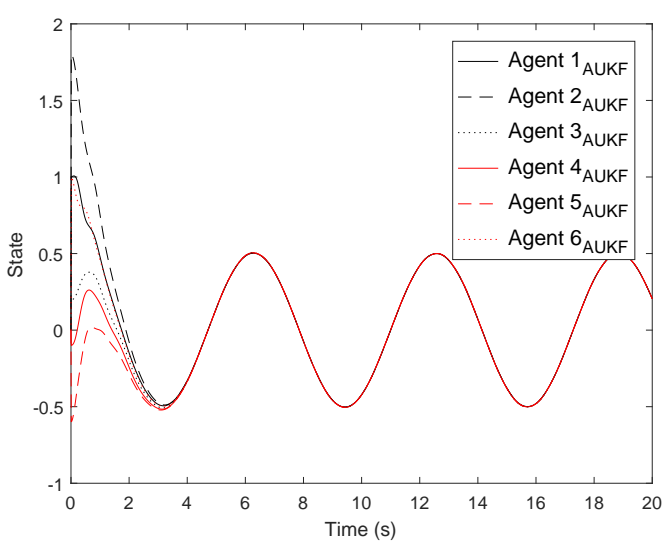

(c) $\hat{\mathrm{x}}_{i 1_{A U K F}}$

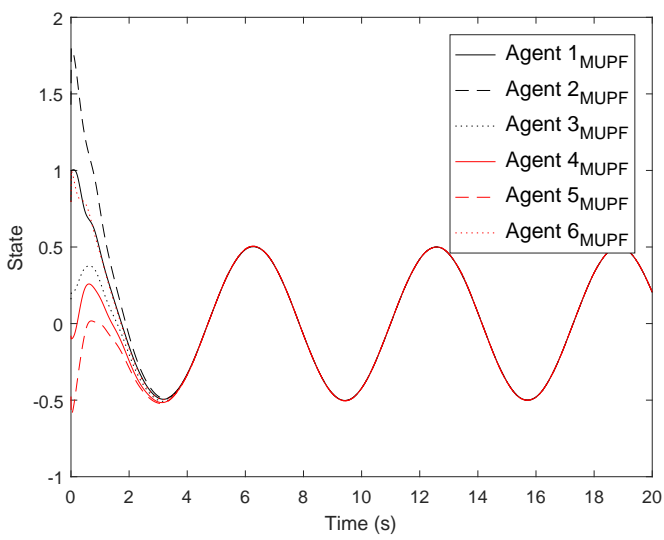

(e) $\hat{\mathrm{x}}_{i 1_{M U P F}}$

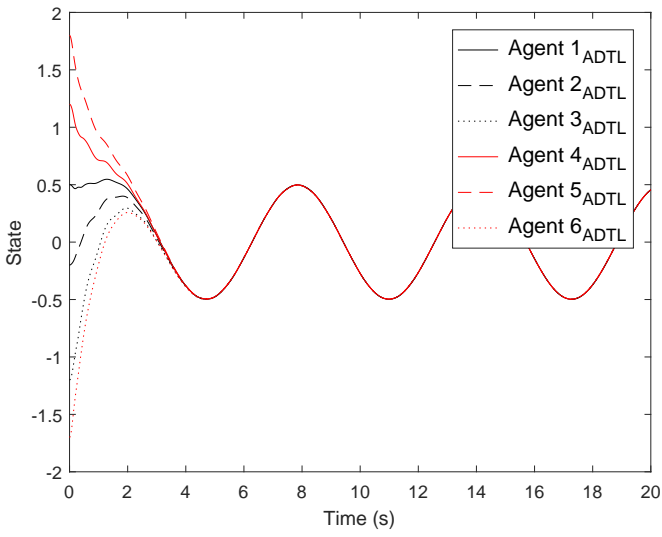

(b) $\hat{\mathrm{x}}_{i 2_{A D T L}}$

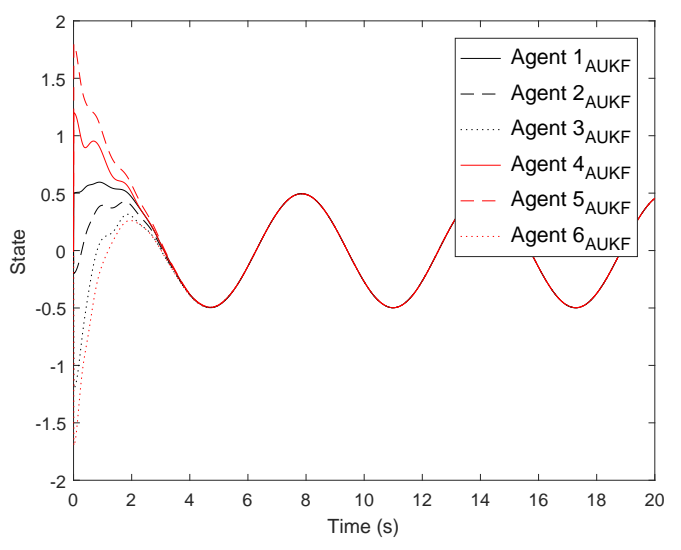

(d) $\hat{\mathrm{x}}_{i 2_{A U K F}}$

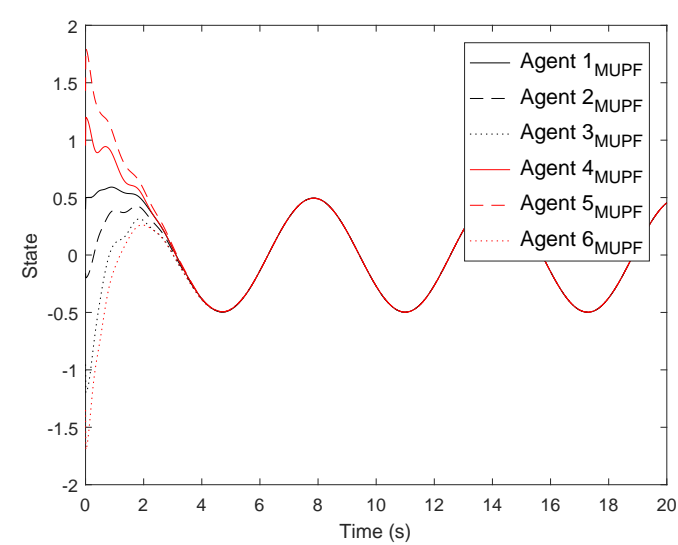

(f) $\hat{\mathrm{x}}_{i 2_{M U P F}}$

Figure 5.3: State estimates $\hat{\mathrm{x}}_{i}=\left(\hat{\mathrm{x}}_{i 1}, \hat{\mathrm{x}}_{i 2}\right)^{T}(i=1,2, \cdots, 6)$. 


\subsection{Simulation Results and Discussion}

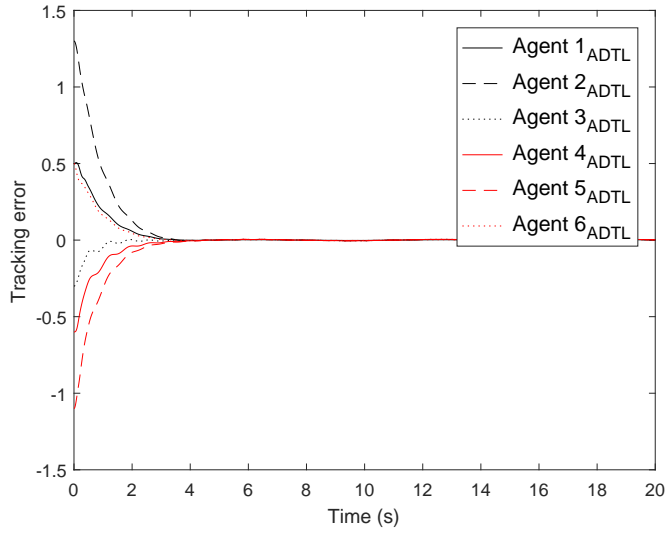

(a) $e_{\mathrm{x} i 1_{A D T L}}$

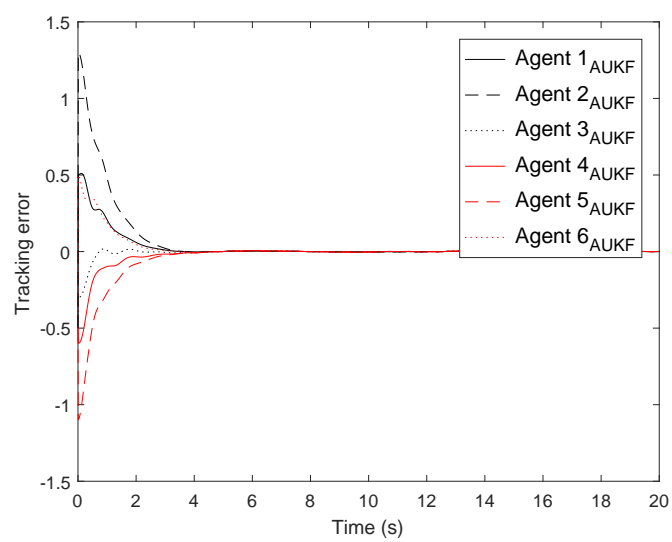

(c) $e_{\mathrm{x} i 1_{A U K F}}$

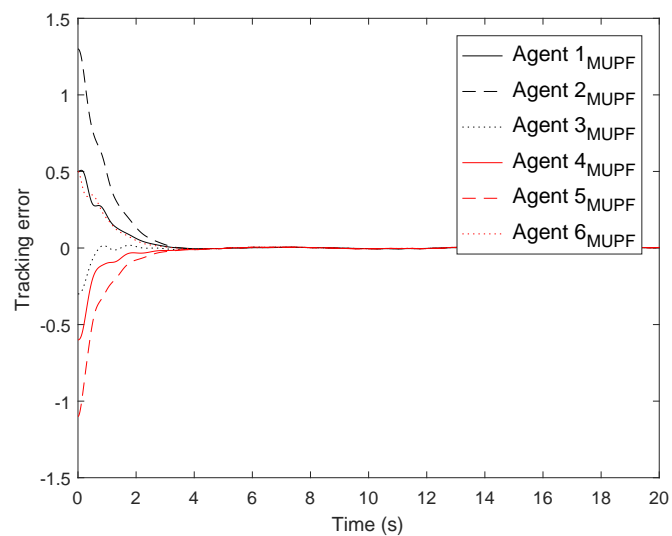

(e) $e_{\mathrm{x} i 1_{M U P F}}$

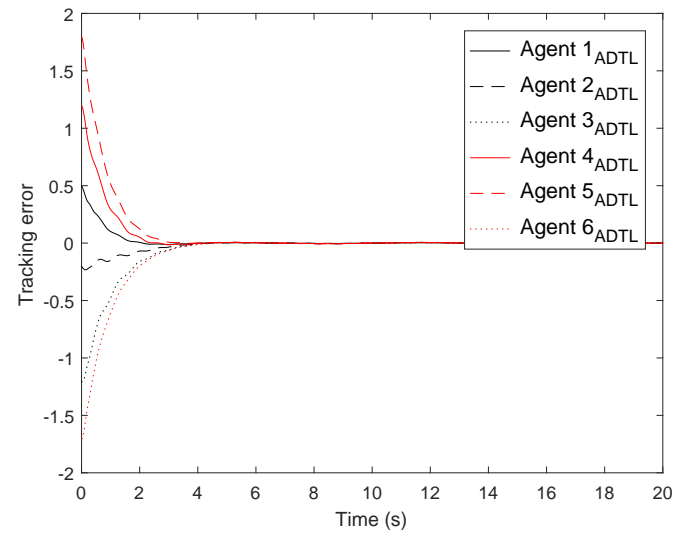

(b) $e_{\mathrm{x} i 2_{A D T L}}$

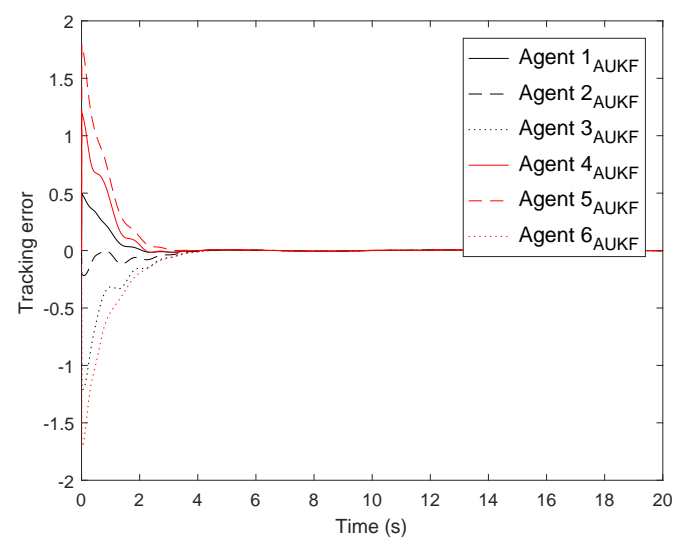

(d) $e_{\mathrm{x} i 2_{A U K F}}$

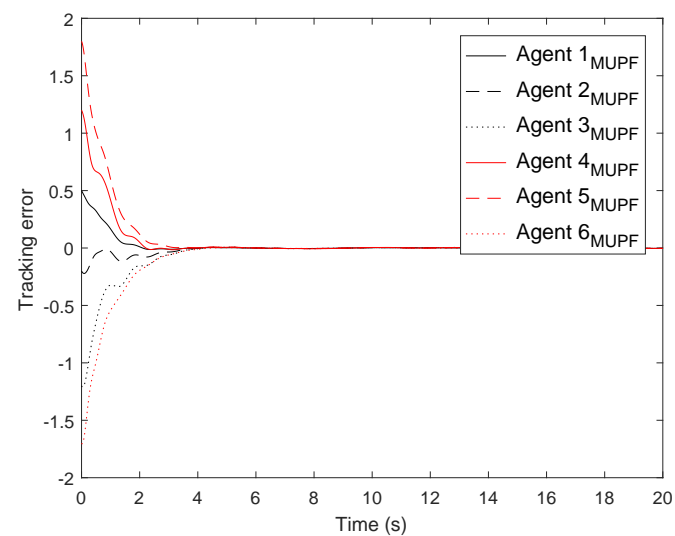

(f) $e_{\mathrm{x} i 2_{M U P F}}$

Figure 5.4: Tracking error $e_{i}=\left(e_{\mathrm{x} i 1}, e_{\mathrm{x} i 2}\right)^{T}(i=1,2, \cdots, 6)$. 
Chapter 5. Online Estimation and Control of Multi-Agent Systems
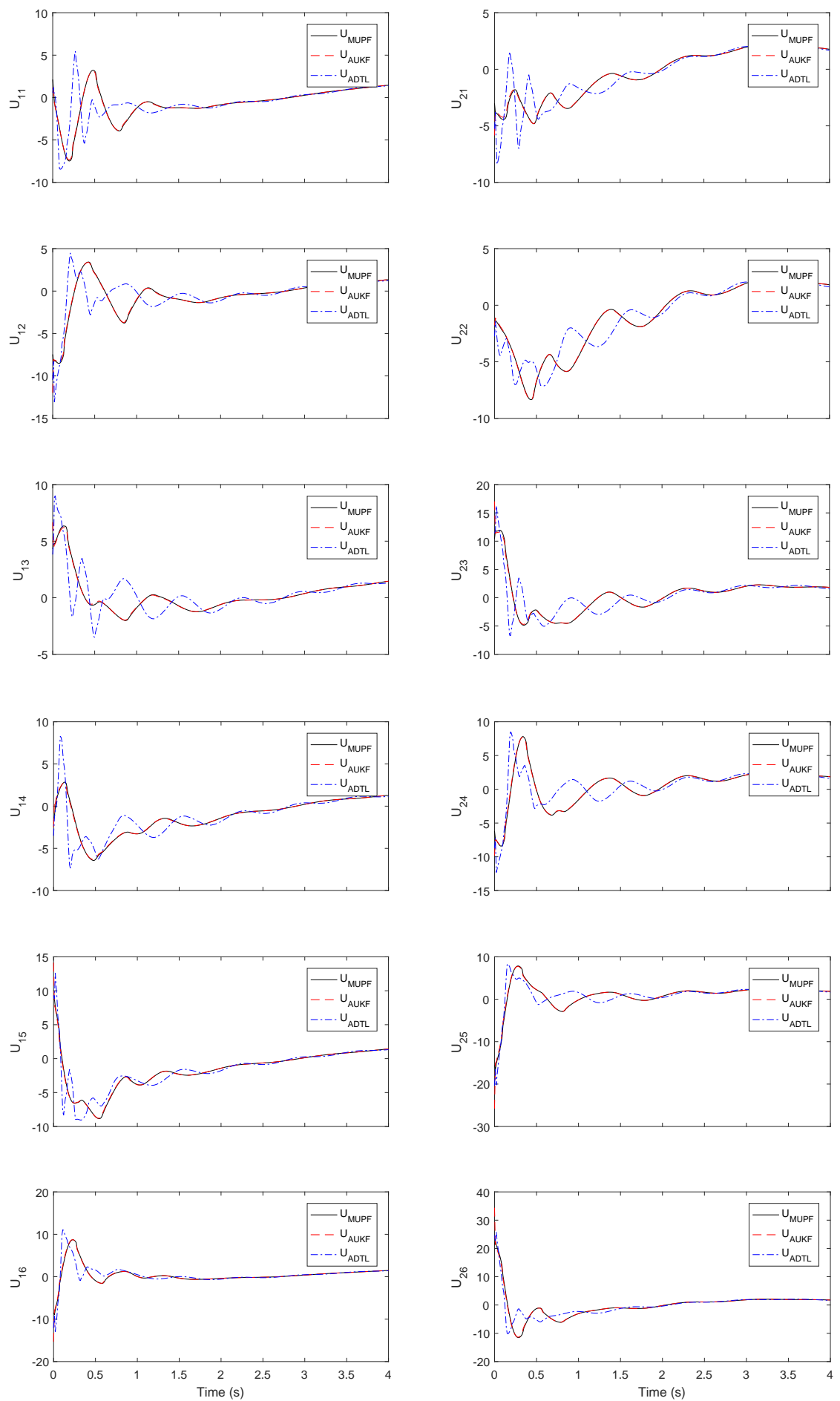

Figure 5.5: Control input under fault-free scenario $u_{i}=\left(u_{i 1}, u_{i 2}\right)^{T}(i=1,2, \cdots, 6)$. 


\subsection{Simulation Results and Discussion}

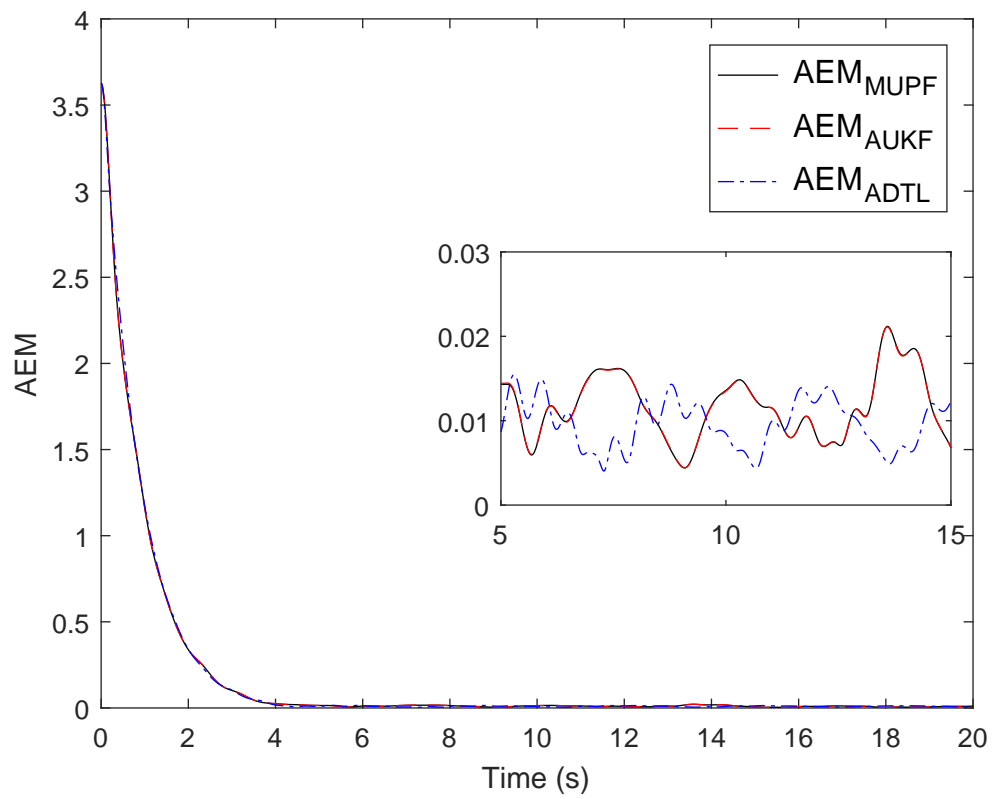

(a) AEM

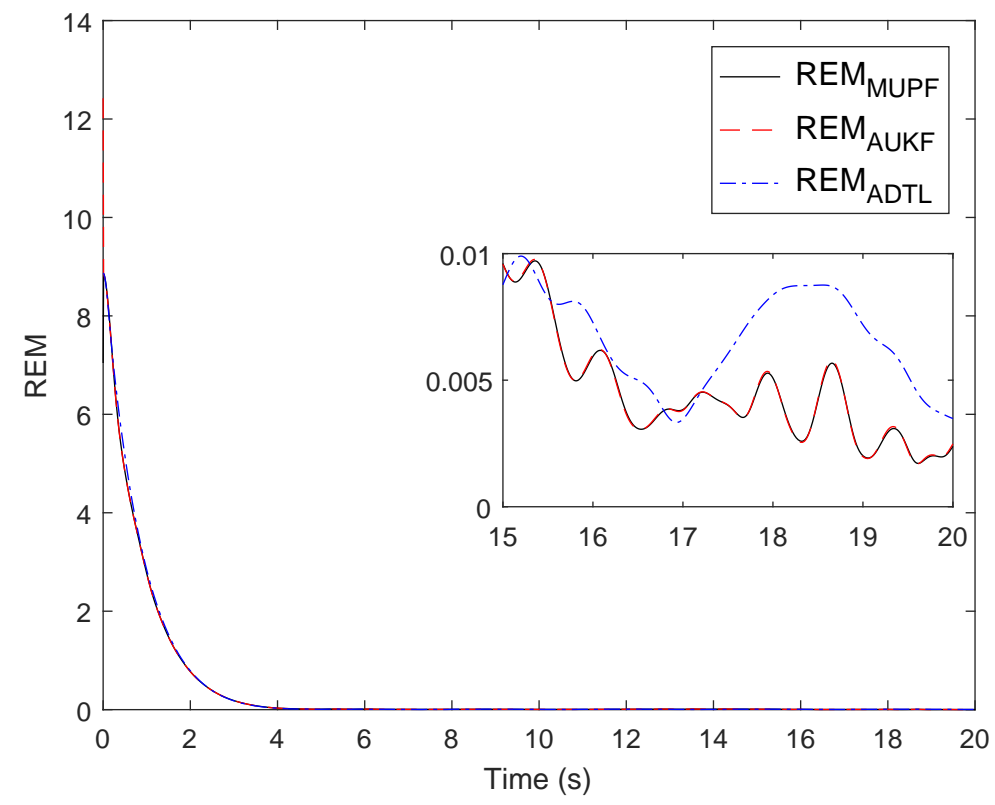

(b) REM

Figure 5.6: Absolute error metric and relative error metric under fault-free scenario. 


\section{Chapter 5. Online Estimation and Control of Multi-Agent Systems}

\subsubsection{Uncertainty in dynamics model}

In the following case, uncertainty is introduced in the multi-agent dynamic models (Equation 5.30) at four different intervals during a 20-seconds simulation time. The simulation reflected changes in the magnitude of the multi-agent dynamics rather than changes in the frequency. Initial assumption is that no fault occurs at the interval between $0<t \leqslant 2$. At this interval the dynamic model is modeled as Equation (5.30). The first fault occurs at the interval between $2<t \leqslant 10$. The second fault occurs at the interval between $10<t \leqslant 15$. Finally, in the last interval between $10<t \leqslant 20$, the multi-agent dynamics is modeled as Equation (5.30). Table 5.3 presents the fault time and the dynamics models.

Table 5.3: Uncertainty in dynamic system.

\begin{tabular}{|l|l|}
\hline \hline Time [sec] & Dynamic model \\
\hline \hline $0<t \leqslant 2$ & $f_{i}\left(\mathrm{x}_{i}, \mathrm{v}_{i}\right)=\left(\begin{array}{c}4 \mathrm{x}_{i 2} \sin \left(\frac{\pi}{4}+\frac{h_{1 i} \mathrm{v}_{i 1}}{2}\right) \\
4 \mathrm{x}_{i 1} \cos \left(\frac{h_{2 i} \mathrm{v}_{i 2}}{2}\right)\end{array}\right)$ \\
\hline $2<t \leqslant 10$ & $f_{i}\left(\mathrm{x}_{i}, \mathrm{v}_{i}\right)=\left(\begin{array}{c}12 \mathrm{x}_{i 2} \sin \left(\frac{\pi}{4}+\frac{h_{1 i} \mathrm{v}_{i 1}}{2}\right) \\
12 \mathrm{x}_{i 1} \cos \left(\frac{h_{2 i} \mathrm{v}_{i 2}}{2}\right)\end{array}\right)$ \\
\hline $10<t \leqslant 15$ & $f_{i}\left(\mathrm{x}_{i}, \mathrm{v}_{i}\right)=\left(\begin{array}{c}5 \mathrm{x}_{i 2} \sin \left(\frac{\pi}{4}+\frac{h_{1 i} \mathrm{v}_{i 1}}{2}\right) \\
-4 \mathrm{x}_{i 1} \cos \left(\frac{h_{2 i} \mathrm{v}_{i 2}}{2}\right)\end{array}\right)$ \\
\hline $15<t \leqslant 20$ & $f_{i}\left(\mathrm{x}_{i}, \mathrm{v}_{i}\right)=\left(\begin{array}{c}4 \mathrm{x}_{i 2} \sin \left(\frac{\pi}{4}+\frac{h_{1 i} \mathrm{v}_{i 1}}{2}\right) \\
4 \mathrm{x}_{i 1} \cos \left(\frac{h_{2 i} \mathrm{v}_{i 2}}{2}\right)\end{array}\right)$ \\
\hline \hline
\end{tabular}

Figures 5.7-5.11 present the simulation results for the adaptive unscented Kalman filter, marginalized unscented particle filter, and the adaptive law under uncertainty in the multi-agent dynamic model. Figure 5.7 shows the estimation results of the uncertain dynamics model $\hat{f}_{i}=\left(\hat{f}_{i 1}, \hat{f}_{i 2}\right)^{T}(i=1,2, \ldots, 6)$. Figure 5.8 present the agent's state estimates $\hat{\mathrm{x}}_{i}=\left(\hat{\mathrm{x}}_{i 1}, \hat{\mathrm{x}}_{i 2}\right)^{T}(i=1,2, \ldots, 6)$; where, Figure $5.8(\mathrm{a})$-(b), are the state estimates using the adaptive law, Figure 5.8 (c)-(d), are the state estimates using the adaptive unscented Kalman filter, and Figure 5.8 (e)-(f), are the state estimates using the marginalized unscented particle filter. Figure 5.9 present the agent's tracking error under uncertainty in the dynamic model $e_{i}=\left(e_{i 1}, e_{i 2}\right)^{T}(i=1,2, \ldots, 6)$; where, Figure 5.9 (a)-(b), are the 


\subsection{Simulation Results and Discussion}

tracking errors using the adaptive law, Figure 5.9 (c)-(d), are the tracking errors using the adaptive unscented Kalman filter, and Figure 5.9 (e)-(f), are the tracking errors using the marginalized unscented particle filter. Figure 5.10 show the control law $u_{i}=\left(u_{i 1}, u_{i 2}\right)^{T}$ $(i=1,2, \cdots, 6)$. Finally, the weights of the third-order Chebyshev neural network are presented in Appendix C.2. 


\section{Chapter 5. Online Estimation and Control of Multi-Agent Systems}
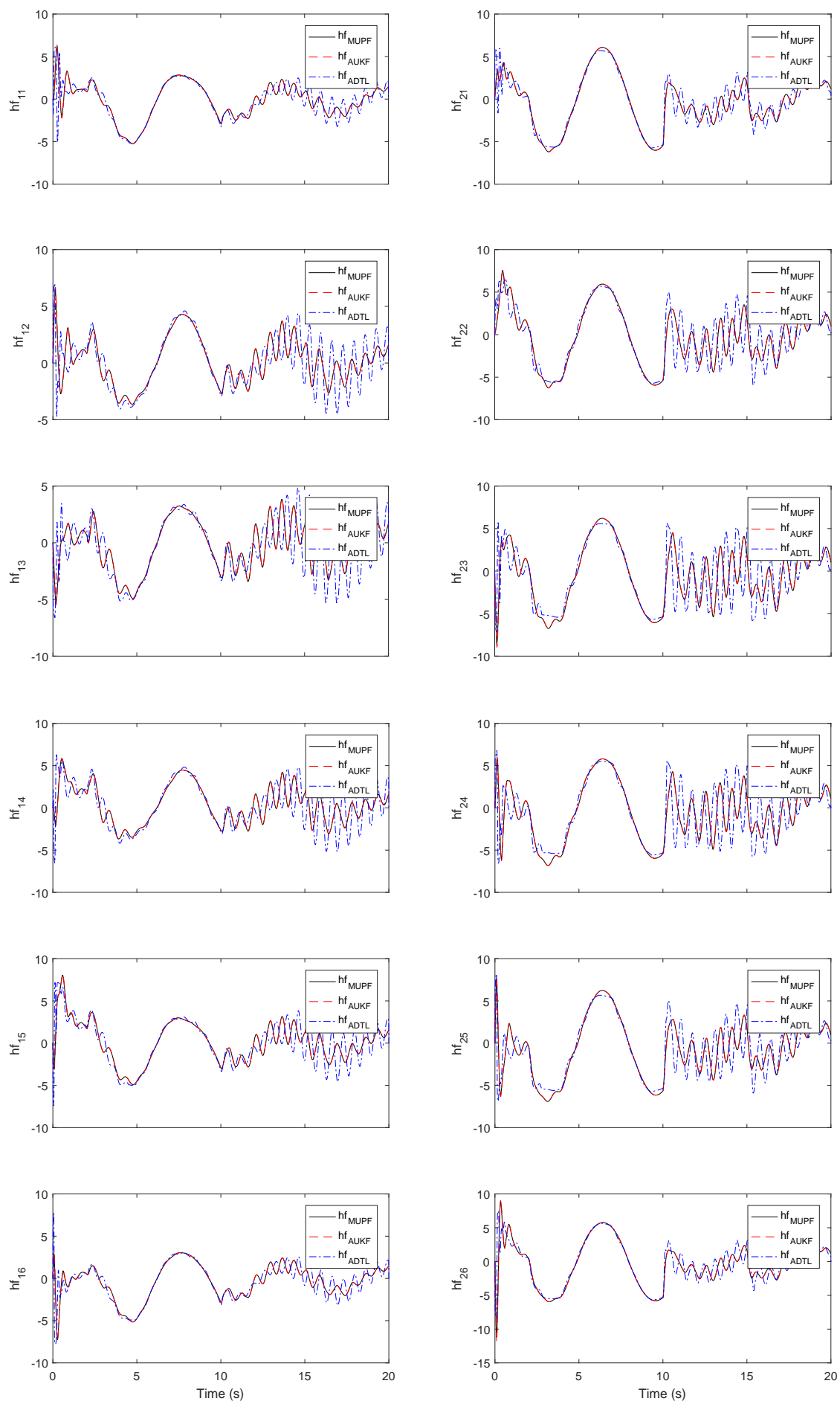

Figure 5.7: Estimation of uncertain dynamic model $\hat{f}_{i}=\left(\hat{f}_{i 1}, \hat{f}_{i 2}\right)^{T}(i=1,2, \ldots, 6)$. 


\subsection{Simulation Results and Discussion}

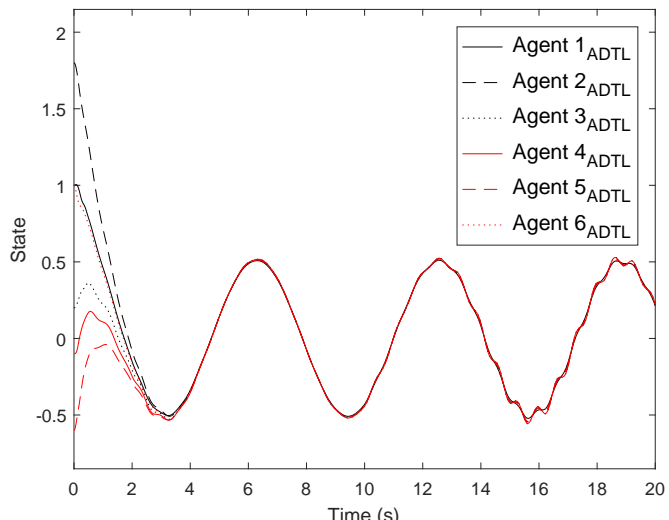

(a) $\hat{\mathrm{x}}_{i 1_{A D T L}}$

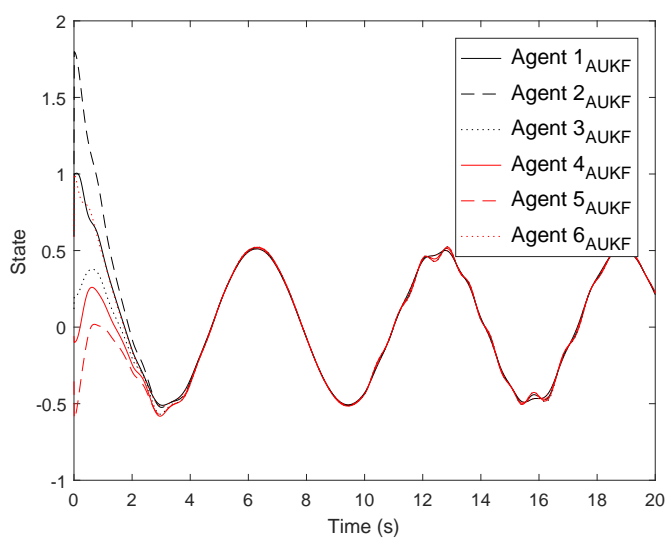

(c) $\hat{\mathrm{x}}_{i 1_{A U K F}}$

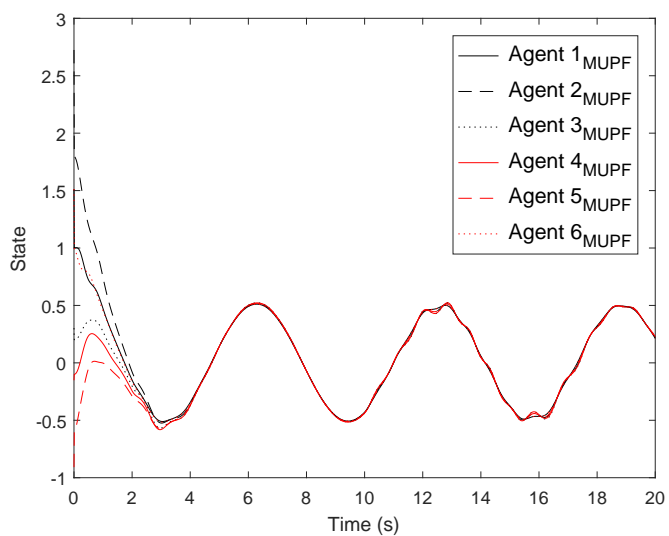

(e) $\hat{\mathrm{x}}_{i 1_{M U P F}}$

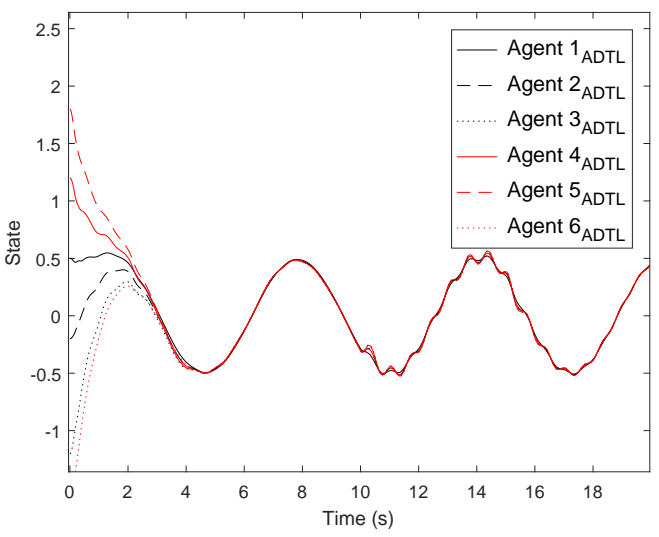

(b) $\hat{\mathrm{x}}_{i 2 A D T L}$

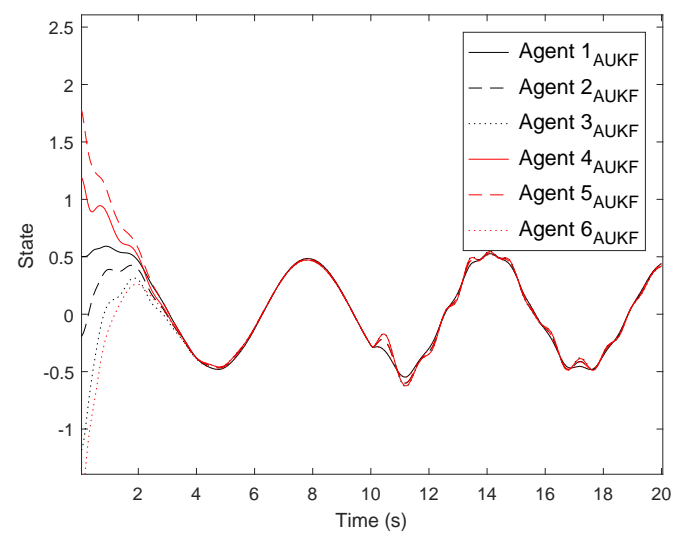

(d) $\hat{\mathrm{x}}_{i 2_{A U K F}}$

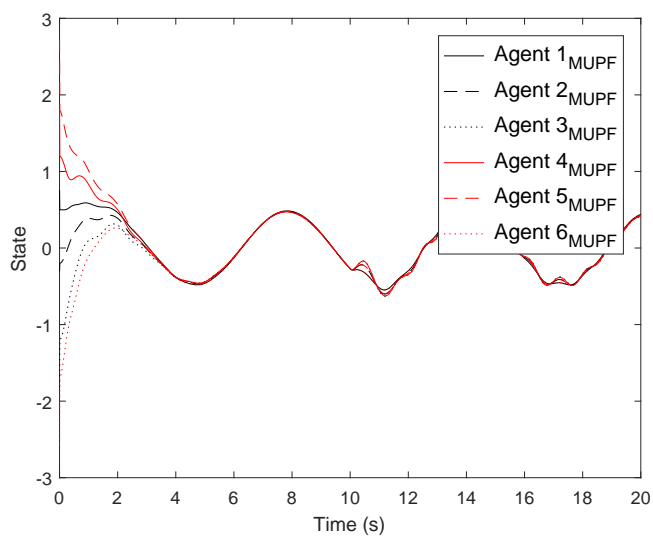

(f) $\hat{\mathbf{x}}_{i 2_{M U P F}}$

Figure 5.8: State estimates under uncertainty in dynamics model $\hat{\mathrm{x}}_{i}=\left(\hat{\mathrm{x}}_{i 1}, \hat{\mathrm{x}}_{i 2}\right)^{T}(i=$ $1,2, \cdots, 6)$. 


\section{Chapter 5. Online Estimation and Control of Multi-Agent Systems}

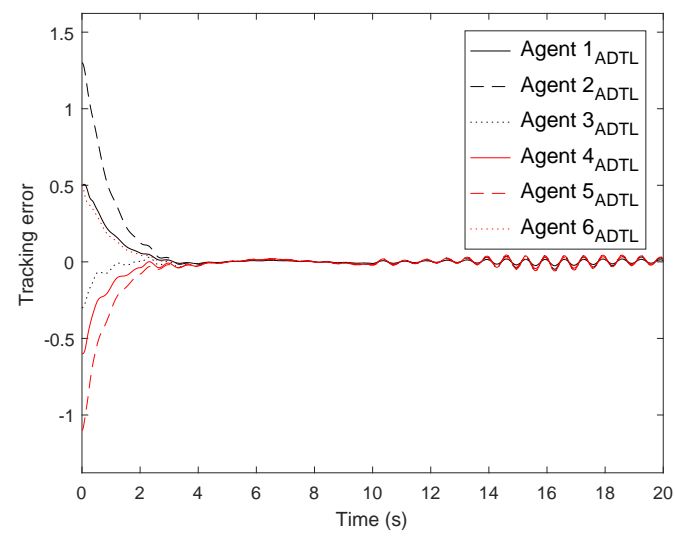

(a) $e_{\mathrm{x} i 1_{A D T L}}$

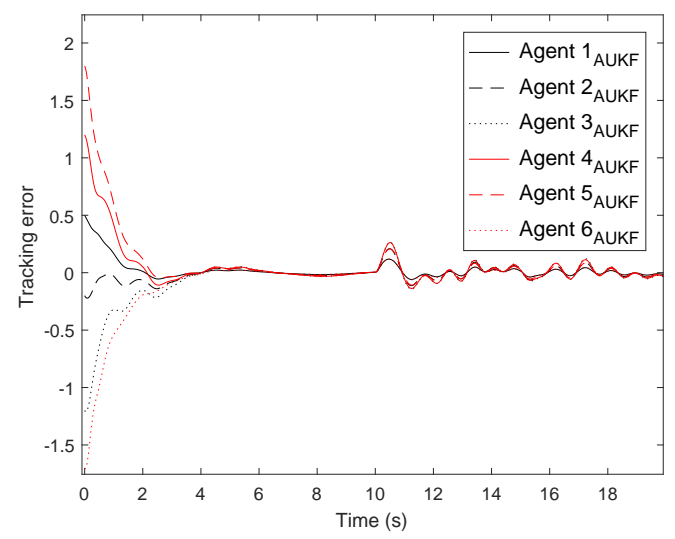

(c) $e_{\mathrm{x} i 1_{A U K F}}$

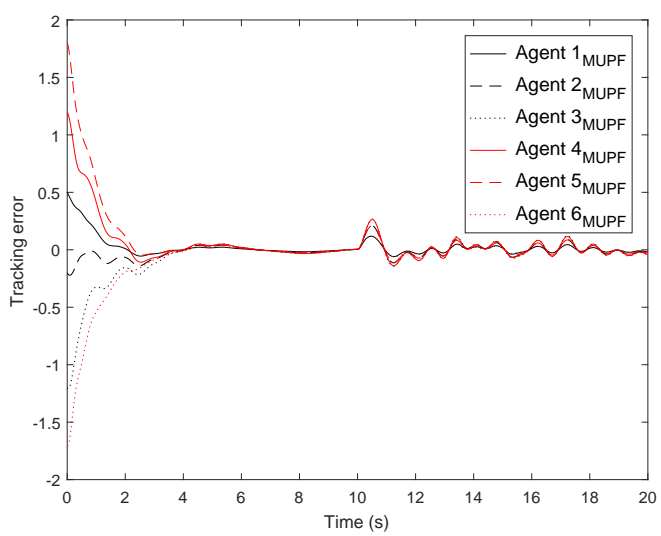

(e) $e_{x i 1_{M U P F}}$

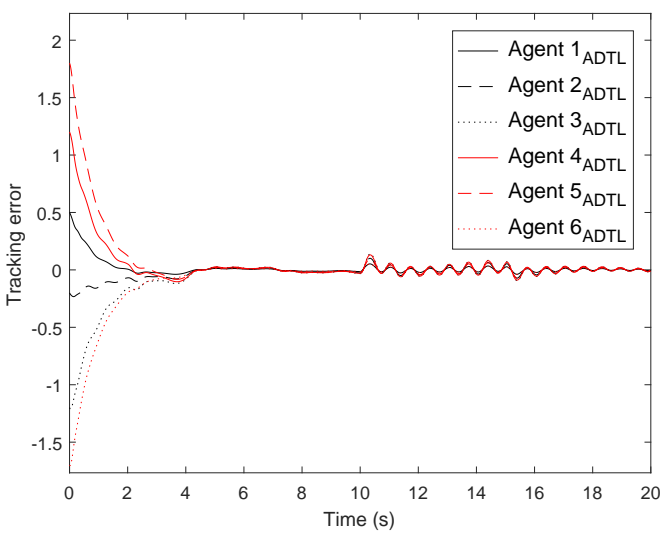

(b) $e_{\mathrm{x} i 2_{A D T L}}$

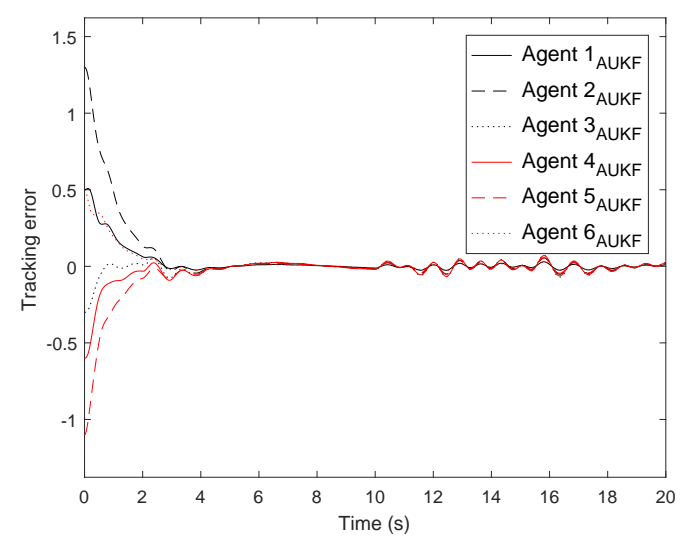

(d) $e_{\mathrm{x} i 22_{A U K F}}$

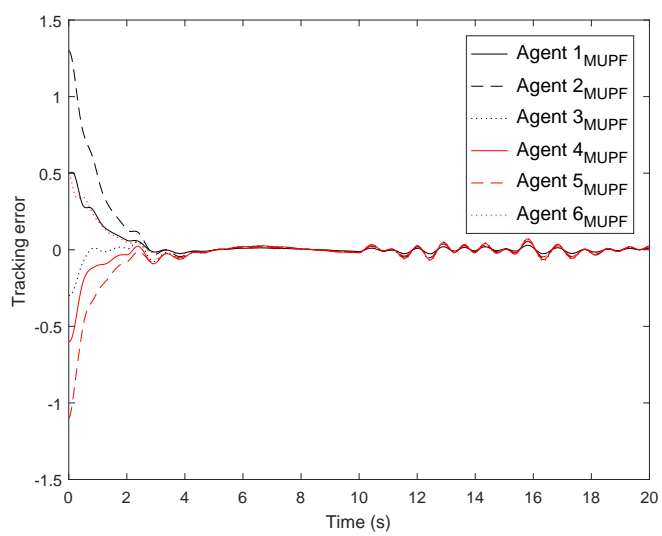

(f) $e_{\mathrm{x} i 2_{M U P F}}$

Figure 5.9: Tracking error under uncertainty in dynamics model $e_{i}=\left(e_{\mathrm{x} i 1}, e_{\mathrm{x} i 2}\right)^{T}(i=$ $1,2, \cdots, 6)$. 


\subsection{Simulation Results and Discussion}
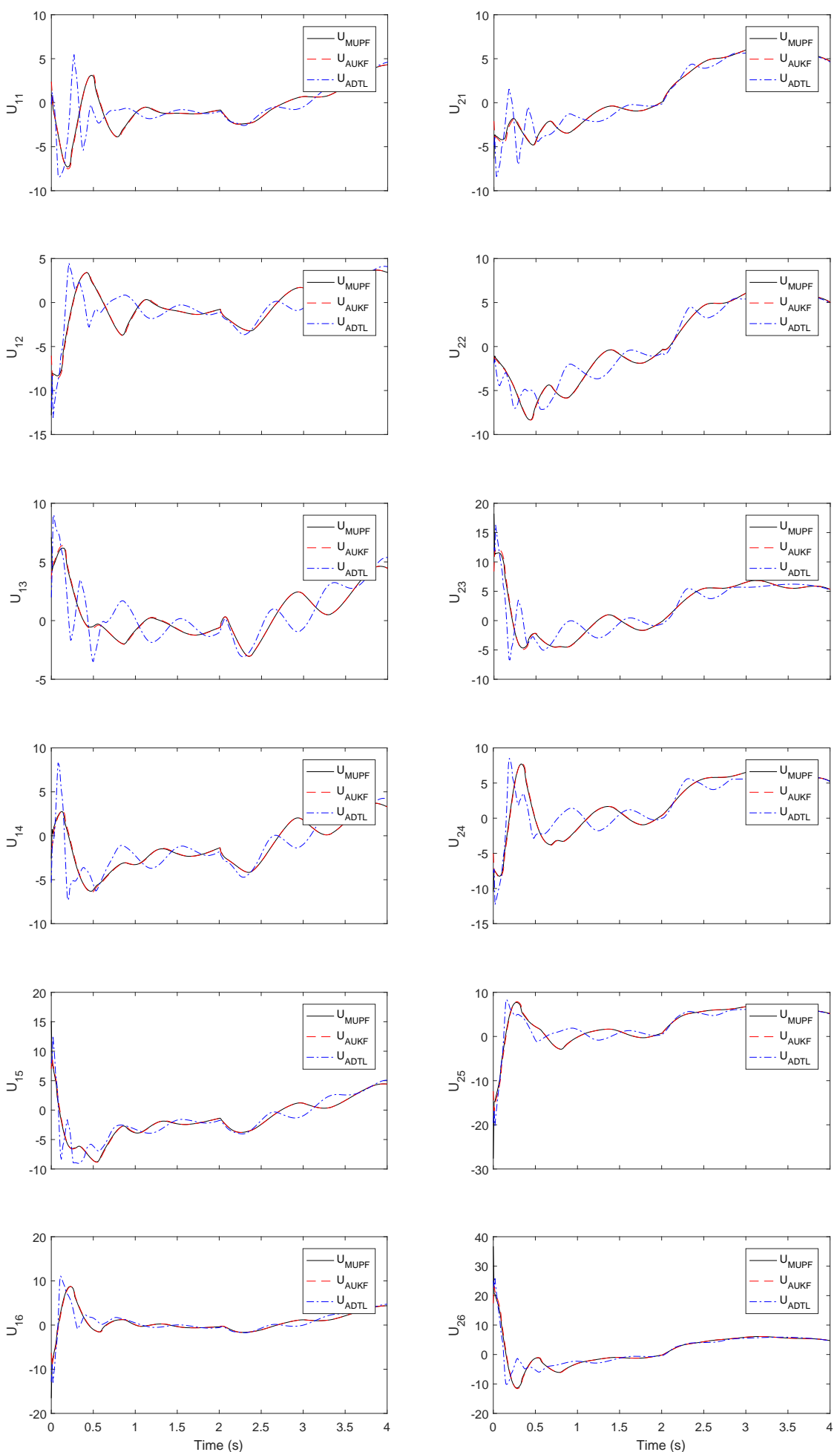

Figure 5.10: Control input under uncertainty in dynamics model $u_{i}=\left(u_{i 1}, u_{i 2}\right)^{T}(i=$ $1,2, \cdots, 6)$. 
Chapter 5. Online Estimation and Control of Multi-Agent Systems

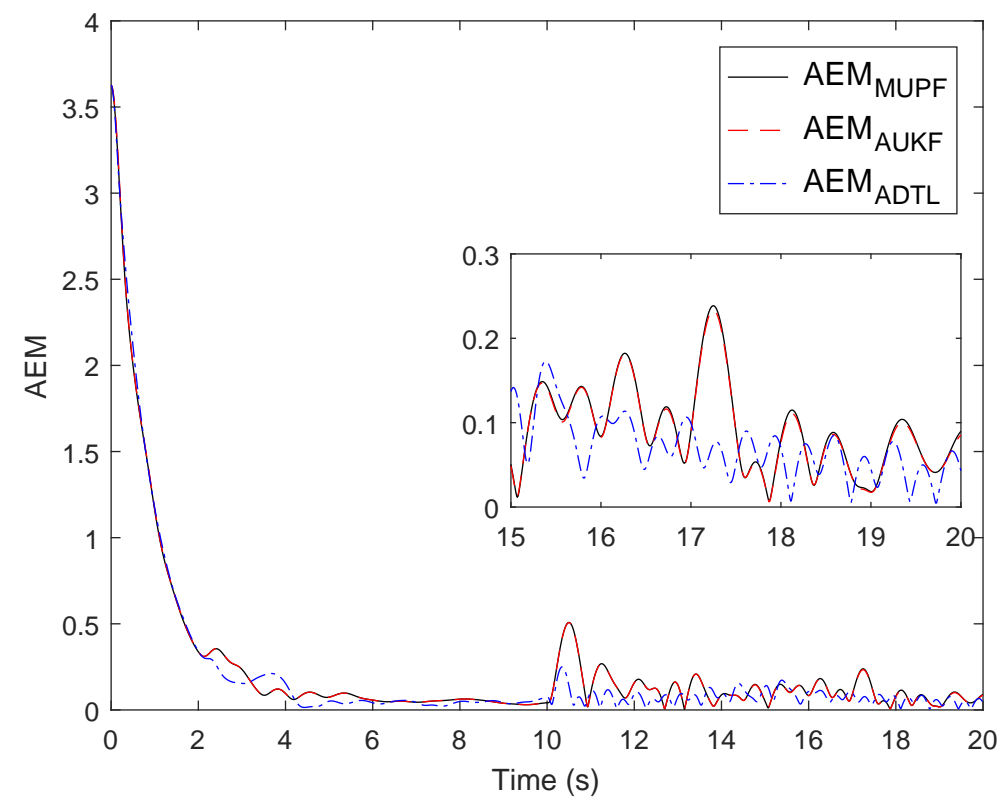

(a) AEM

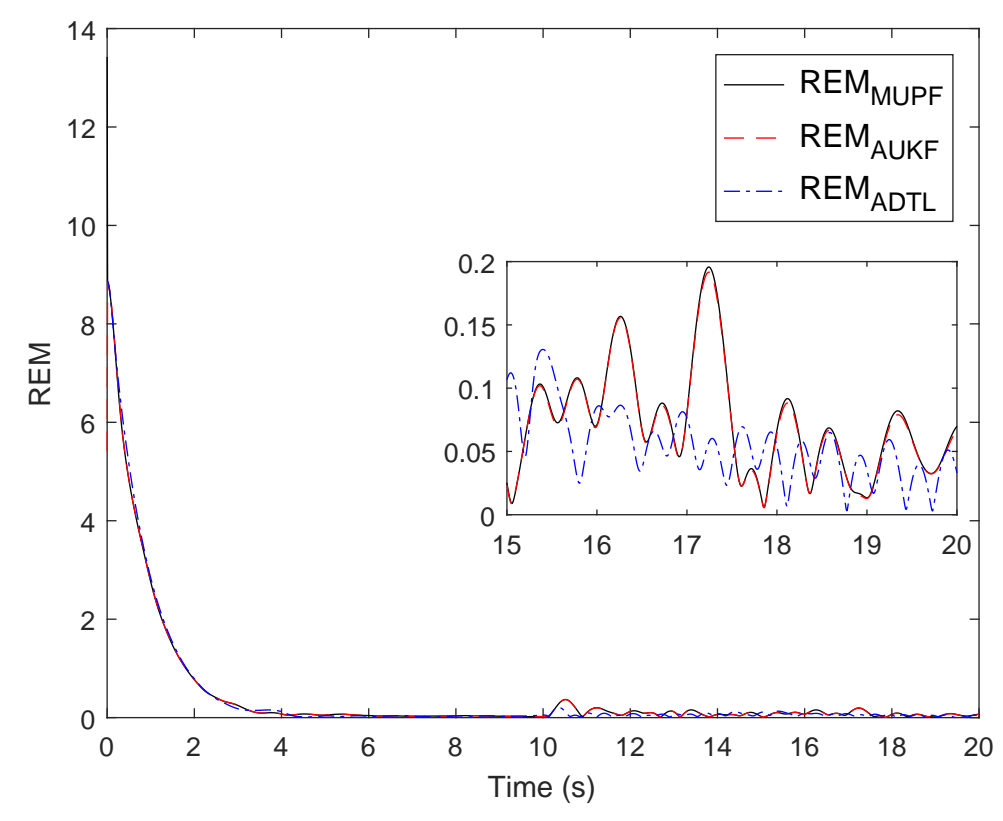

(b) REM

Figure 5.11: Absolute error metric and relative error metric under uncertainty in dynamics model. 


\subsection{Simulation Results and Discussion}

\subsubsection{External disturbances}

The second-order dynamic equation which describes the multi-agent system, includes a bounded external disturbances (Equation 5.2). In the following case, the external disturbances are altered at four different intervals during a 20-seconds simulation time. In first interval, between $0<t \leqslant 5$, the external disturbances are expressed as $\vartheta_{i}=$ $0.1\left(\sin \left(\frac{t}{2}\right), \cos \left(\frac{t}{2}\right)\right)^{T}$. The second interval, between $5<t \leqslant 10$, reflect an increase in magnitude of the external disturbances. In this interval the magnitude of the external disturbances is given by, $\vartheta_{i}=8\left(\sin \left(\frac{t}{2}\right), \cos \left(\frac{t}{2}\right)\right)^{T}$. The third interval, between $10<t \leqslant 15$ reflect changes in frequency of the external disturbances. In this interval, the external disturbances are expressed as $\vartheta_{i}=0.1(\sin (2 t), \cos (2 t))^{T}$. Finally, in the last interval, between $10<t \leqslant 20$, the external disturbances are bounded by the expression $\vartheta_{i}=0.1\left(\sin \left(\frac{t}{2}\right), \cos \left(\frac{t}{2}\right)\right)^{T}$. The external disturbances and their corresponding time intervals are presented in Table 5.4.

Table 5.4: External disturbances.

\begin{tabular}{|l|ll|}
\hline \hline Time [sec] & External disturbances & \\
\hline \hline $0<t \leqslant 5$ & $\vartheta_{i}=d_{i}\left(\sin \left(\frac{t}{2}\right), \cos \left(\frac{t}{2}\right)\right)^{T}$ & where $d_{i}=0.1$ \\
\hline $5<t \leqslant 10$ & $\vartheta_{i}=d_{i}\left(\sin \left(\frac{t}{2}\right), \cos \left(\frac{t}{2}\right)\right)^{T}$ & where $d_{i}=8$ \\
\hline $10<t \leqslant 15$ & $\vartheta_{i}=d_{i}(\sin (2 t), \cos (2 t))^{T}$ & where $d_{i}=0.1$ \\
\hline $15<t \leqslant 20$ & $\vartheta_{i}=d_{i}\left(\sin \left(\frac{t}{2}\right), \cos \left(\frac{t}{2}\right)\right)^{T}$ & where $d_{i}=0.1$ \\
\hline \hline
\end{tabular}

Figures 5.12-5.16 present the simulation results for the adaptive unscented Kalman filter, marginalized unscented particle filter, and the adaptive law under the presence of external disturbances. Figure 5.12 shows the estimation results of the uncertain dynamic model $\hat{f}_{i}=\left(\hat{f}_{i 1}, \hat{f}_{i 2}\right)^{T}(i=1,2, \ldots, 6)$ under external disturbances. Figure 5.13 present the agent's state estimates $\hat{\mathrm{x}}_{i}=\left(\hat{\mathrm{x}}_{i 1}, \hat{\mathrm{x}}_{i 2}\right)^{T}(i=1,2, \ldots, 6)$; where, Figure 5.13 (a)-(b), are the state estimates using the adaptive law, Figure 5.13 (c)-(d), are the state estimates using the adaptive unscented Kalman filter, and Figure 5.13 (e)-(f), are the state estimates using 


\section{Chapter 5. Online Estimation and Control of Multi-Agent Systems}

the marginalized unscented particle filter. Figure 5.14 present the agent's tracking error under external disturbances $e_{i}=\left(e_{i 1}, e_{i 2}\right)^{T}(i=1,2, \ldots, 6)$; where, Figure 5.14 (a)-(b), are the tracking errors using the adaptive law, Figure 5.14 (c)-(d), are the tracking errors using the adaptive unscented Kalman filter, and Figure 5.14 (e)-(f), are the tracking errors using the marginalized unscented particle filter. Figure 5.15 show the control law $u_{i}=\left(u_{i 1}, u_{i 2}\right)^{T}(i=1,2, \cdots, 6)$. Finally, the weights of the third-order Chebyshev neural network are presented in Appendix C.3. 


\subsection{Simulation Results and Discussion}
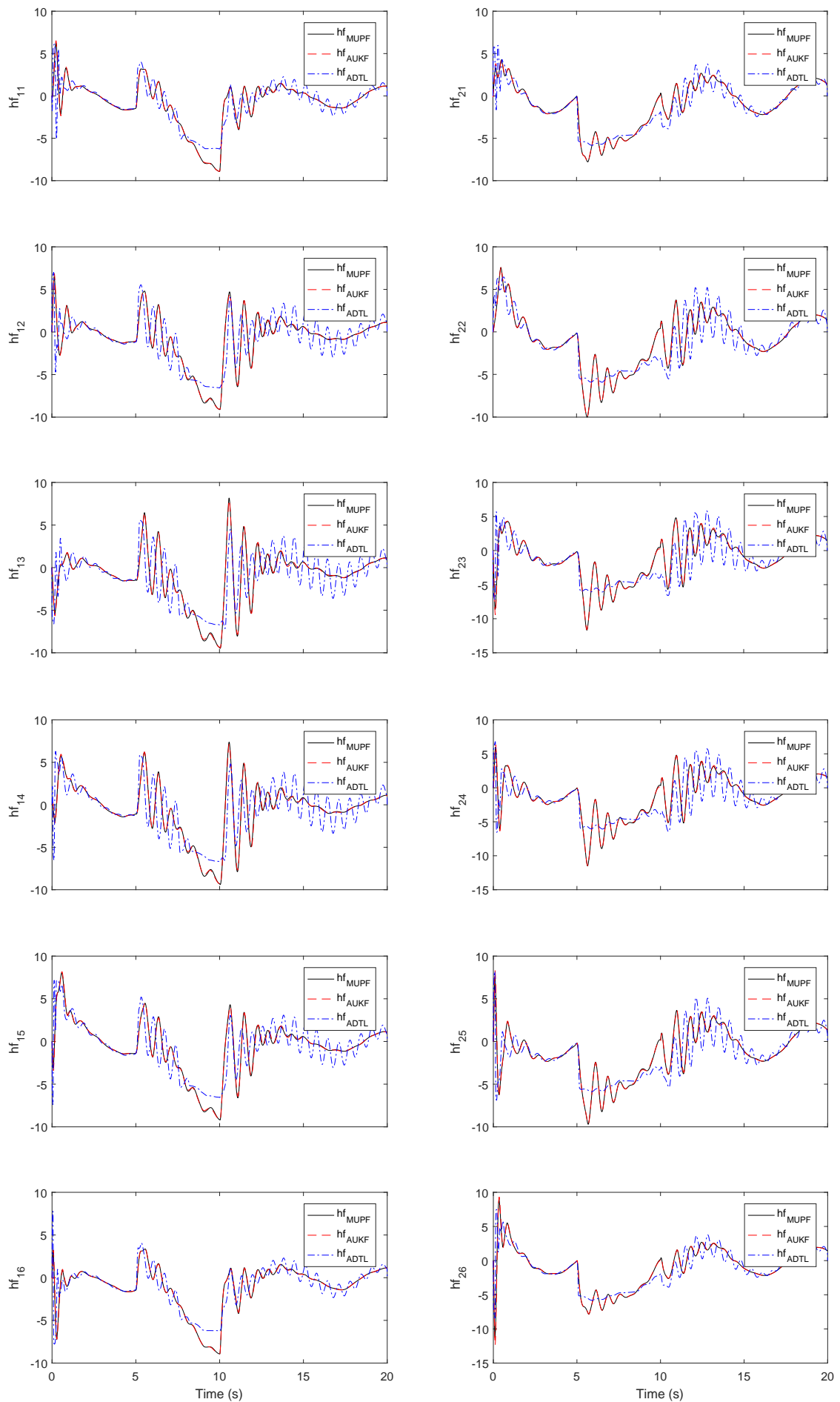

Figure 5.12: Estimation of uncertain dynamics model under external disturbances $\hat{f}_{i}=$ $\left(\hat{f}_{i 1}, \hat{f}_{i 2}\right)^{T}(i=1,2, \ldots, 6)$. 


\section{Chapter 5. Online Estimation and Control of Multi-Agent Systems}

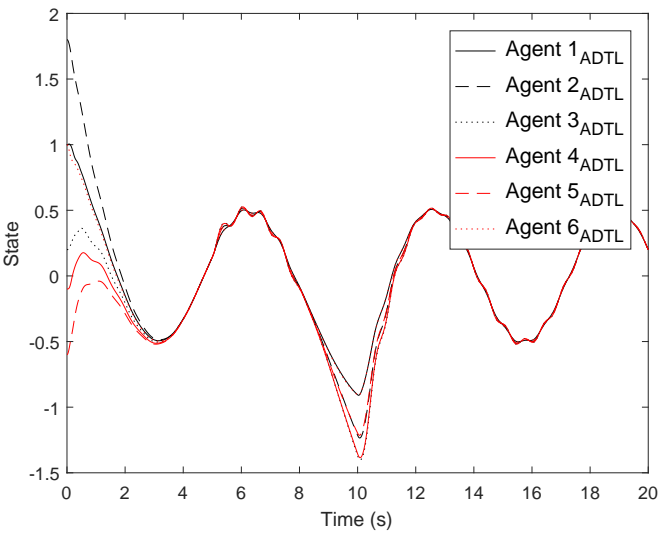

(a) $\hat{\mathrm{x}}_{i 1_{A D T L}}$

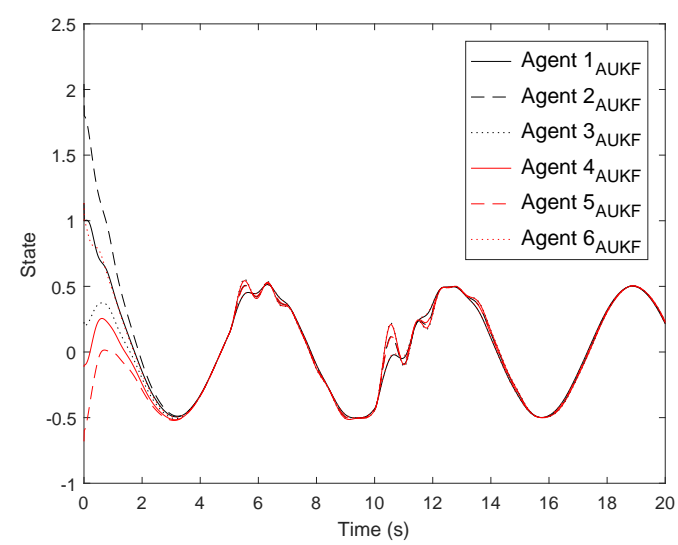

(c) $\hat{\mathrm{x}}_{i 1_{A U K F}}$

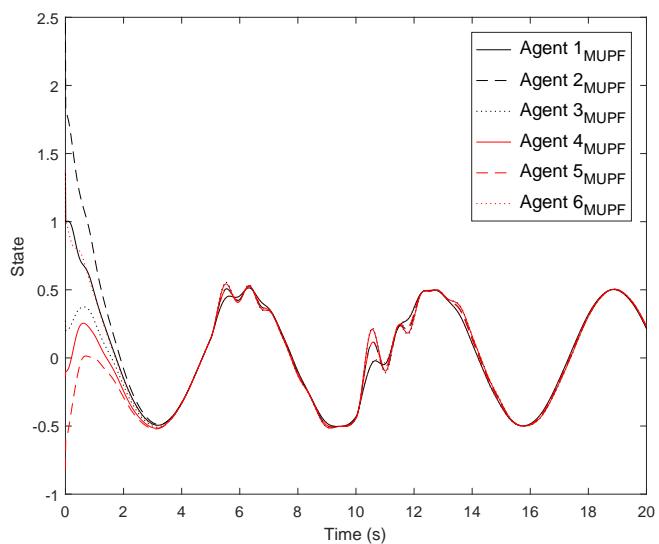

(e) $\hat{\mathrm{x}}_{i 1_{M U P F}}$

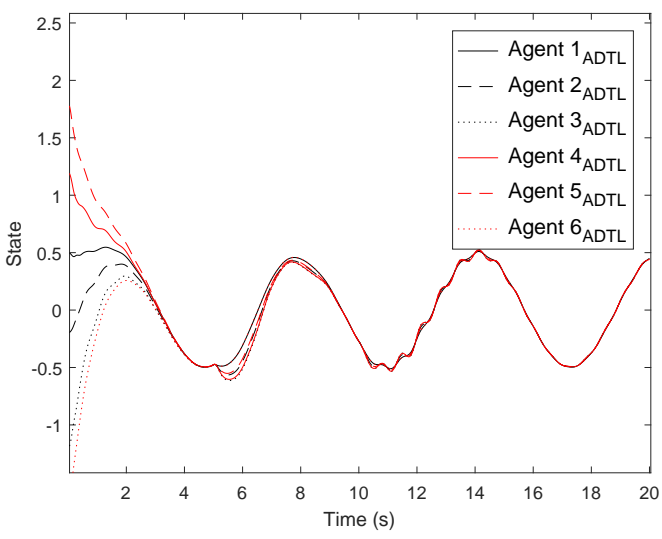

(b) $\hat{\mathrm{x}}_{i 2_{A D T L}}$

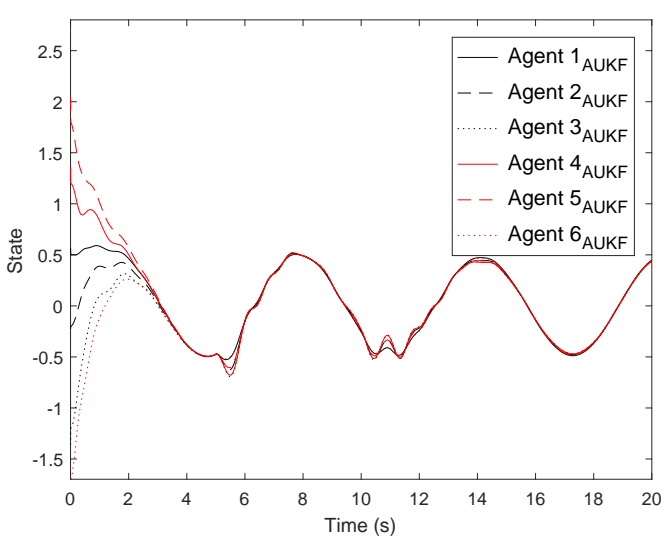

(d) $\hat{\mathrm{x}}_{i 2_{A U K F}}$

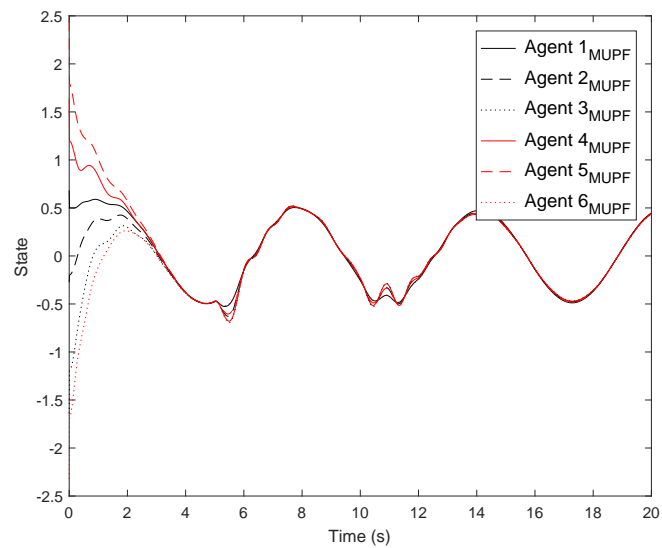

(f) $\hat{\mathrm{x}}_{i 2 M U P F}$

Figure 5.13: State estimates under external disturbances $\hat{\mathrm{x}}_{i}=\left(\hat{\mathrm{x}}_{i 1}, \hat{\mathrm{x}}_{i 2}\right)^{T}(i=1,2, \cdots, 6)$. 


\subsection{Simulation Results and Discussion}

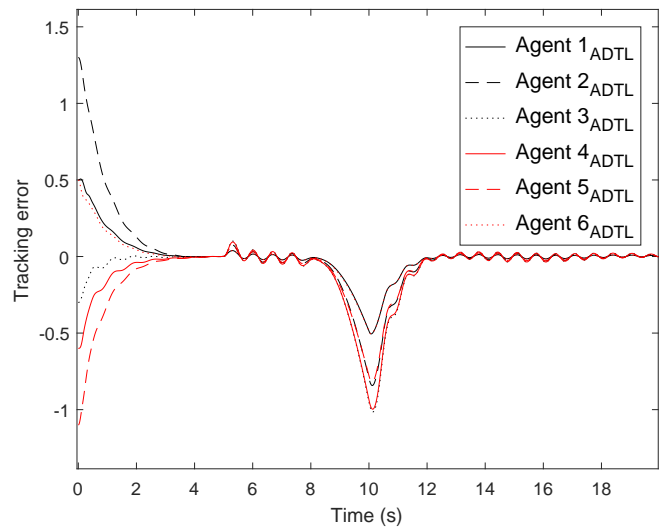

(a) $e_{\mathrm{x} i 1_{A D T L}}$

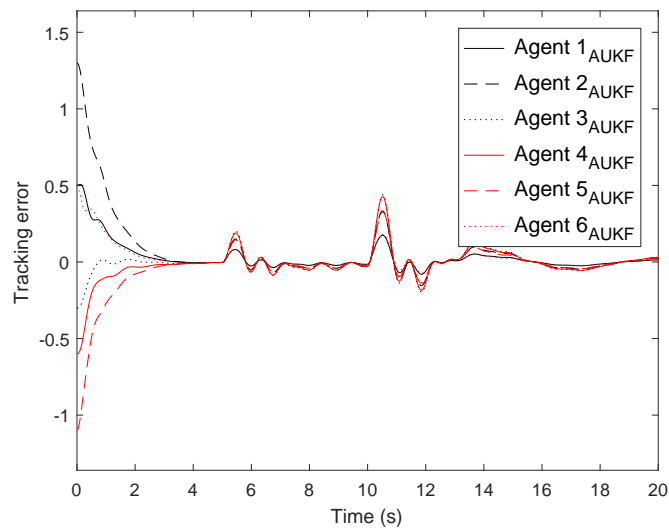

(c) $e_{\mathrm{x} i 1_{A U K F}}$

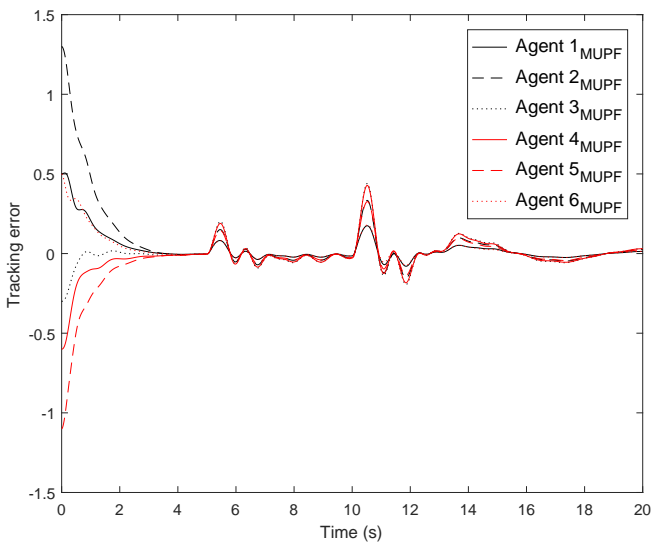

(e) $e_{\mathrm{x} i 1_{M U P F}}$

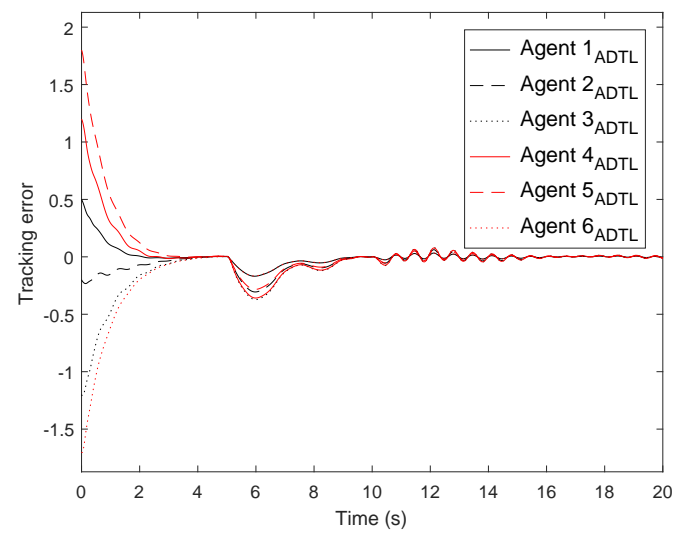

(b) $e_{\mathrm{x} i 2_{A D T L}}$

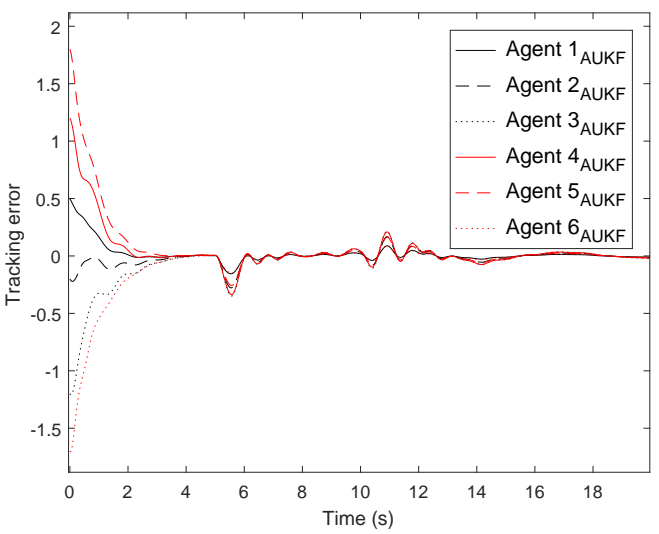

(d) $e_{\mathrm{x} i 2_{A U K F}}$

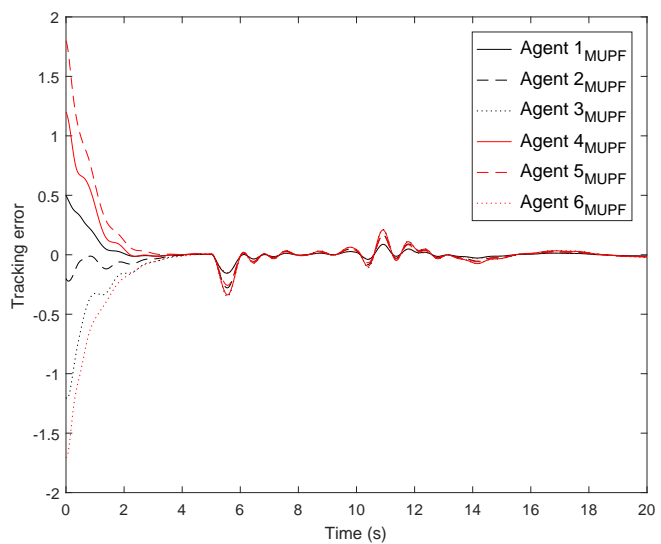

(f) $e_{\mathrm{x} i 2_{M U P F}}$

Figure 5.14: Tracking error under external disturbances $e_{i}=\left(e_{\mathrm{x} i 1}, e_{\mathrm{x} i 2}\right)^{T}(i=1,2, \cdots, 6)$. 


\section{Chapter 5. Online Estimation and Control of Multi-Agent Systems}
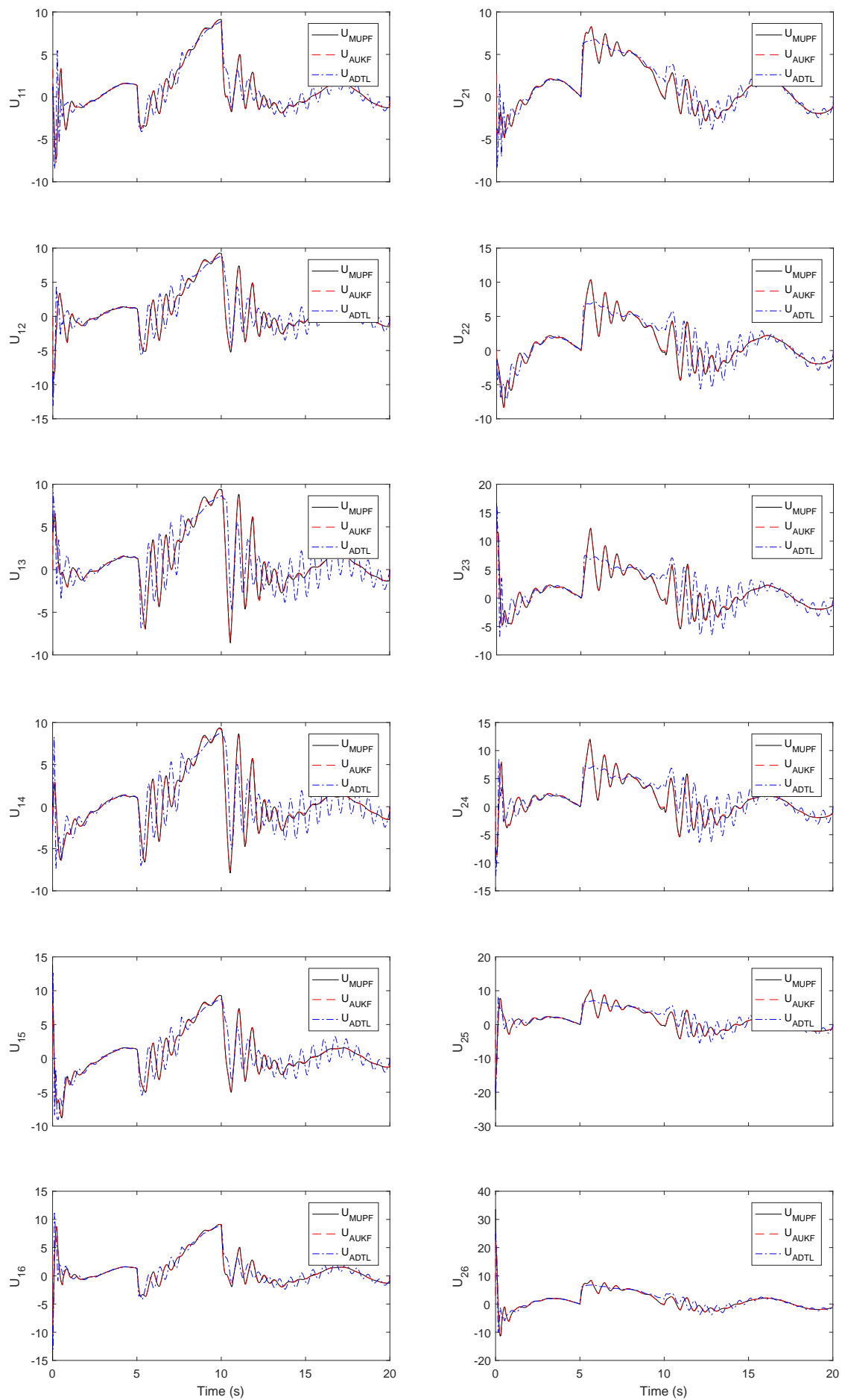

Figure 5.15: Control law under external disturbances $u_{i}=\left(u_{i 1}, u_{i 2}\right)^{T}(i=1,2, \cdots, 6)$. 


\subsection{Simulation Results and Discussion}

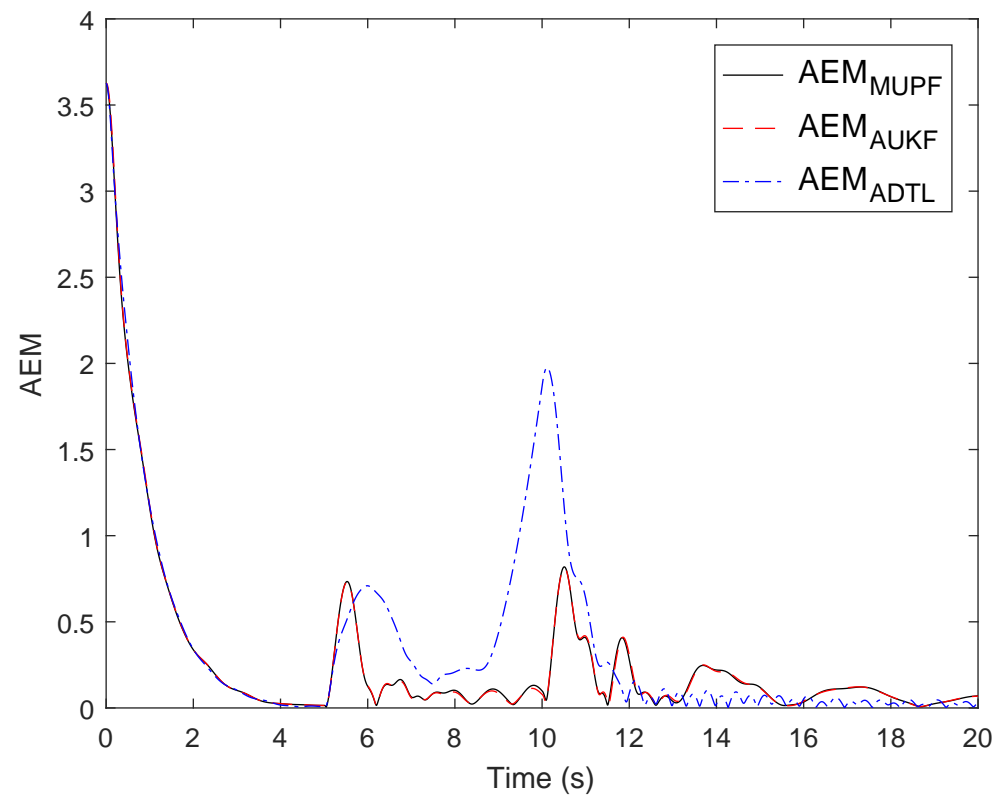

(a) AEM

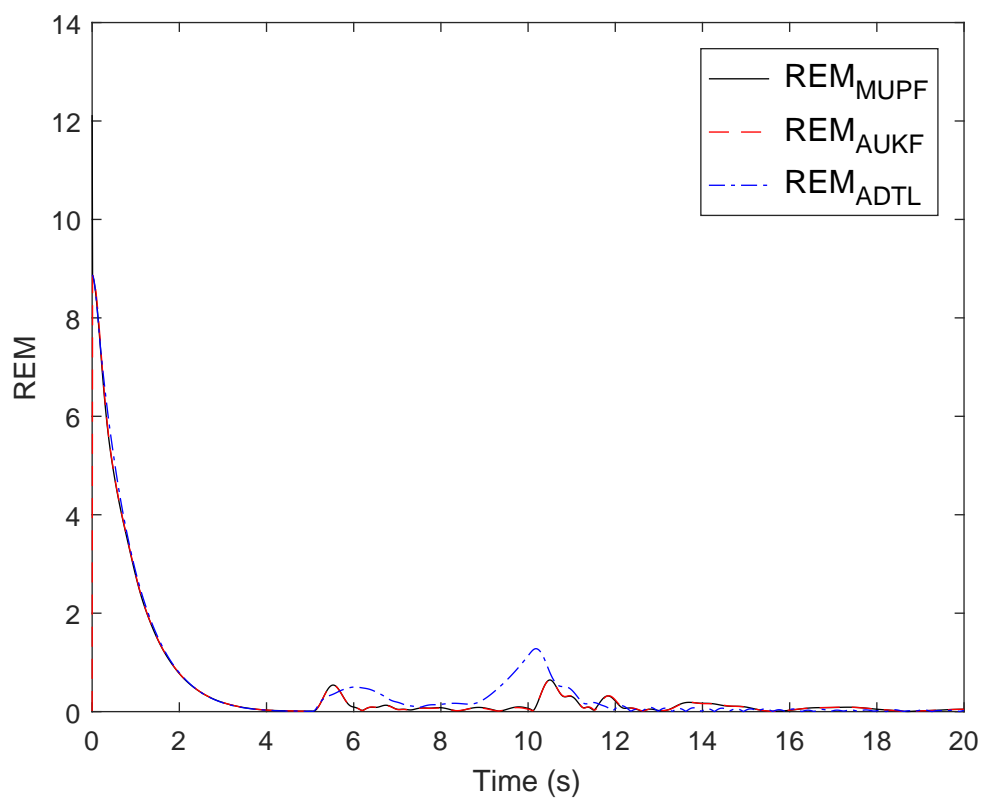

(b) REM

Figure 5.16: Absolute error metric and relative error metric under external disturbances. 


\section{Chapter 5. Online Estimation and Control of Multi-Agent Systems}

\subsubsection{Control command fault}

In the following case, faults in the control command are applied at three intervals during a simulation time of 20 -seconds. The first interval, between $0<t \leqslant 2$, the control command is operating under ideal conditions and is expressed by Equation (5.17). The first fault occurs at $2<t \leqslant 6$; where the fast terminal sliding manifolds $\left(K_{1 i} s_{i}\right.$ and $\left.K_{2 i} s_{i}\right)$ generate a zero control input while the uncertain dynamics model and robust control laws are minimized by a constant negative parameter. In the third interval, between $6<t \leqslant 20$, the control command is recovered and expressed by Equation (5.17). The control commands and their corresponding time intervals are presented in Table 5.5.

Table 5.5: Constant applied control command.

\begin{tabular}{|c|c|}
\hline Time [sec] & Control command \\
\hline $0<t \leqslant 2$ & $u_{i}=\mathrm{g}_{i}^{-1}\left(-W_{i} \xi_{i}-K_{1 i} s_{i}-K_{2 i} s_{i}-\psi_{i}\right), \quad i=1,2, \cdots, n$ \\
\hline $2<t \leqslant 6$ & $u_{i}=\mathrm{g}_{i}^{-1}\left(-W_{i} \xi_{i}-\psi_{i}\right)\left(\begin{array}{l}-0.0125 \\
-0.0125\end{array}\right)$, \\
\hline $6<t \leqslant 20$ & $u_{i}=\mathrm{g}_{i}^{-1}\left(-W_{i} \xi_{i}-K_{1 i} s_{i}-K_{2 i} s_{i}-\psi_{i}\right), \quad i=1,2, \cdots, n$ \\
\hline
\end{tabular}

Figures 5.17-5.21 present the simulation results for AUKF, MUPF and adaptive law. Figure 5.17 shows the estimation results of the uncertain dynamic model $\hat{f}_{i}=\left(\hat{f}_{i 1}, \hat{f}_{i 2}\right)^{T}(i=$ $1,2, \ldots, 6)$. Figure 5.18 present the agent's state estimates $\hat{\mathrm{x}}_{i}=\left(\hat{\mathrm{x}}_{i 1}, \hat{\mathrm{x}}_{i 2}\right)^{T}(i=1,2, \ldots, 6)$; where, Figure 5.18 (a)-(b), are the state estimates using the adaptive law, Figure 5.18 (c)(d), are the state estimates using the adaptive unscented Kalman filter, and Figure 5.18 (e)(f), are the state estimates using the marginalized unscented particle filter. Figure 5.19 present the agent's tracking error, $e_{i}=\left(e_{i 1}, e_{i 2}\right)^{T}(i=1,2, \ldots, 6)$, under the constant control laws; where, Figure 5.19 (a)-(b), are the tracking errors using the adaptive law, Figure 5.19 (c)-(d), are the tracking errors using the adaptive unscented Kalman filter, and Figure 5.19 (e)-(f), are the tracking errors using the marginalized unscented particle filter. Figure 5.20 and show the control laws $u_{i}=\left(u_{i 1}, u_{i 2}\right)^{T}(i=1,2, \cdots, 6)$. Finally, the weights of Chebyshev neural network are presented in Appendix C.4. 


\subsection{Simulation Results and Discussion}
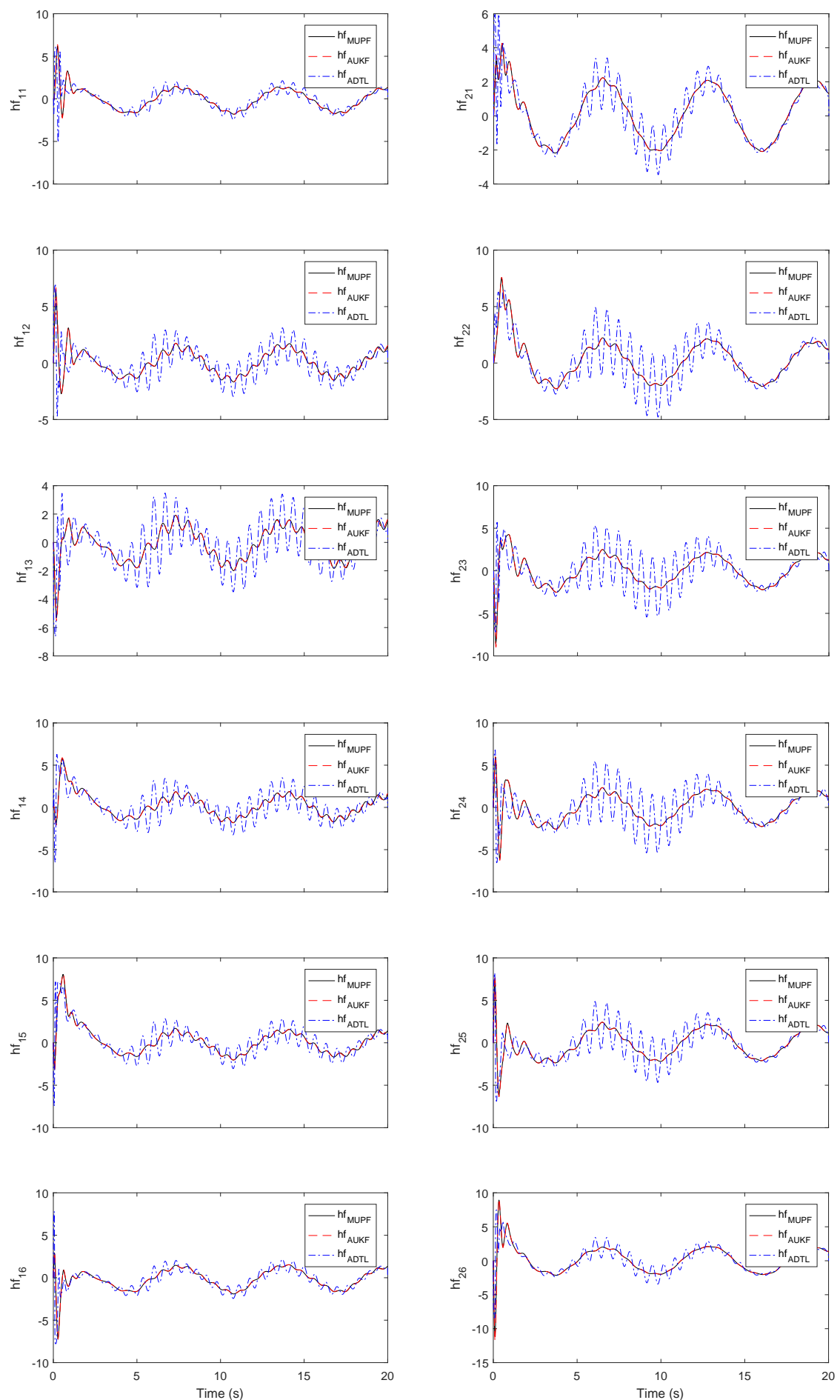

Figure 5.17: Estimation of uncertain dynamics model under a constant control command $\hat{f}_{i}=\left(\hat{f}_{i 1}, \hat{f}_{i 2}\right)^{T}(i=1,2, \ldots, 6)$. 


\section{Chapter 5. Online Estimation and Control of Multi-Agent Systems}

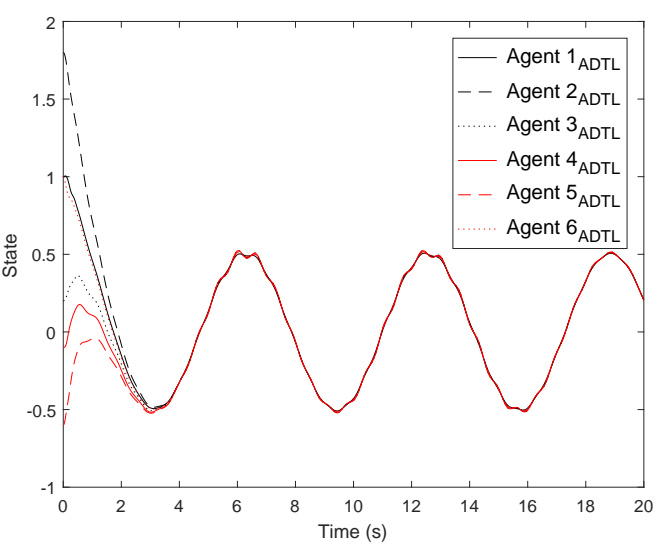

(a) $\hat{\mathrm{x}}_{i 1_{A D T L}}$

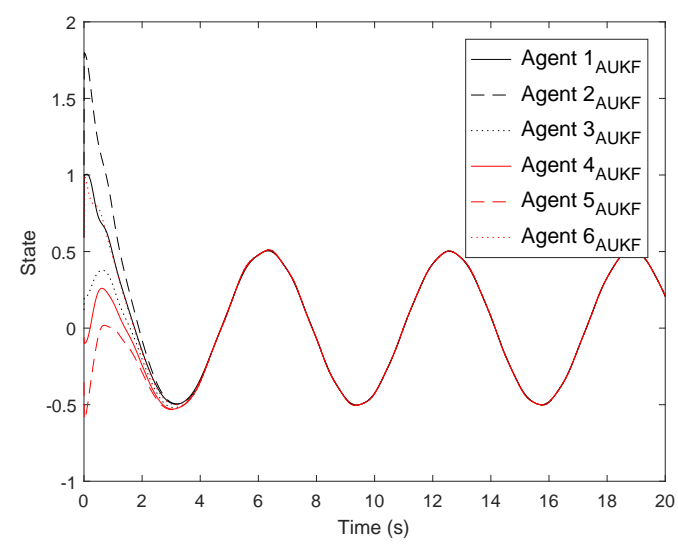

(c) $\hat{\mathrm{x}}_{i 1_{A U K F}}$

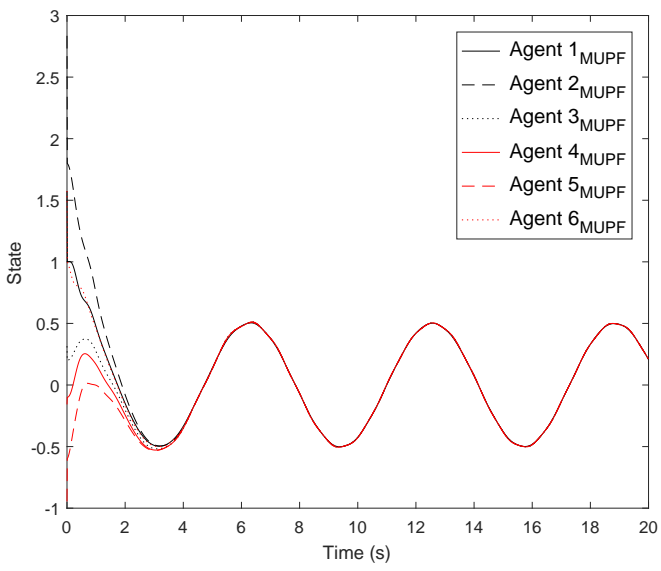

(e) $\hat{\mathrm{x}}_{i 1_{M U P F}}$

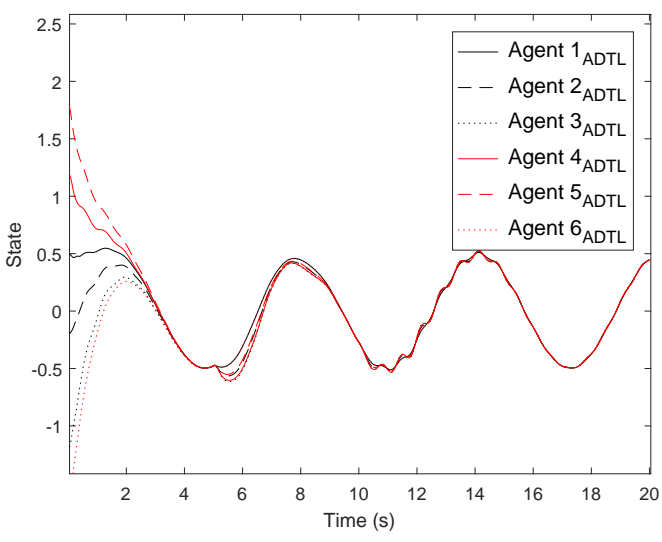

(b) $\hat{\mathrm{x}}_{i 2_{A D T L}}$

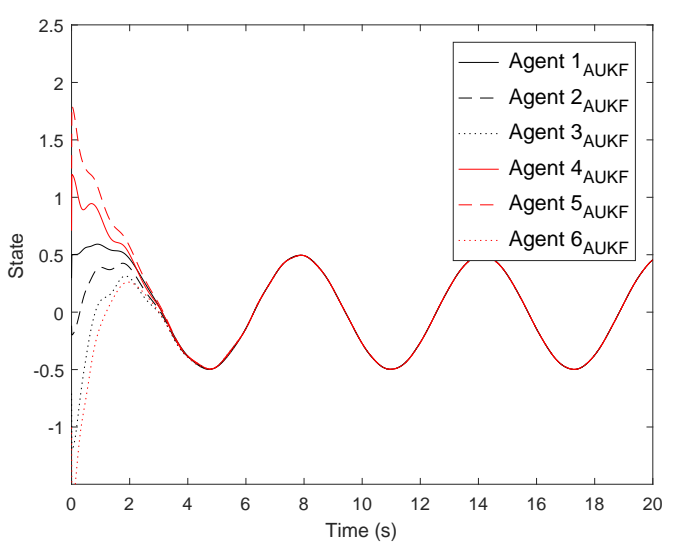

(d) $\hat{\mathrm{x}}_{i 2_{A U K F}}$

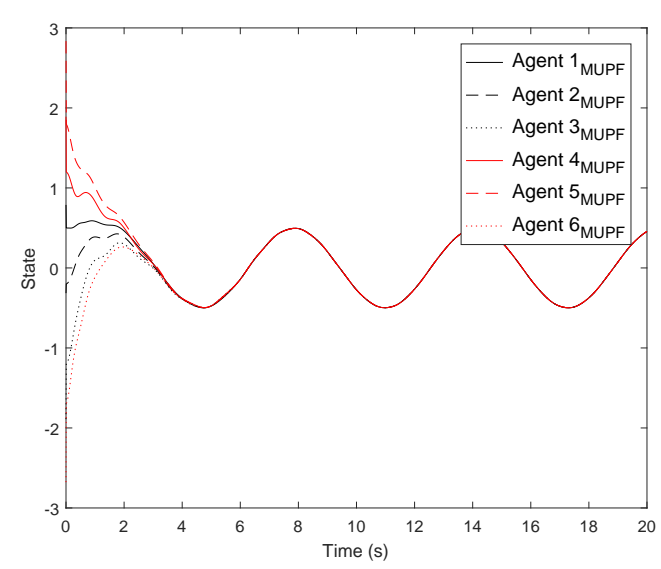

(f) $\hat{\mathrm{x}}_{i 2_{M U P F}}$

Figure 5.18: State estimates under a constant control command $\hat{\mathrm{x}}_{i}=\left(\hat{\mathrm{x}}_{i 1}, \hat{\mathrm{x}}_{i 2}\right)^{T}(i=$ $1,2, \cdots, 6)$. 


\subsection{Simulation Results and Discussion}

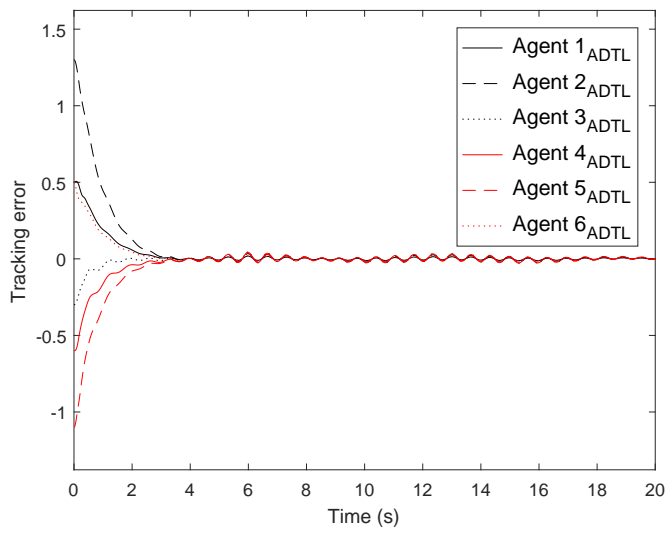

(a) $e_{\mathrm{x} i 1_{A D T L}}$

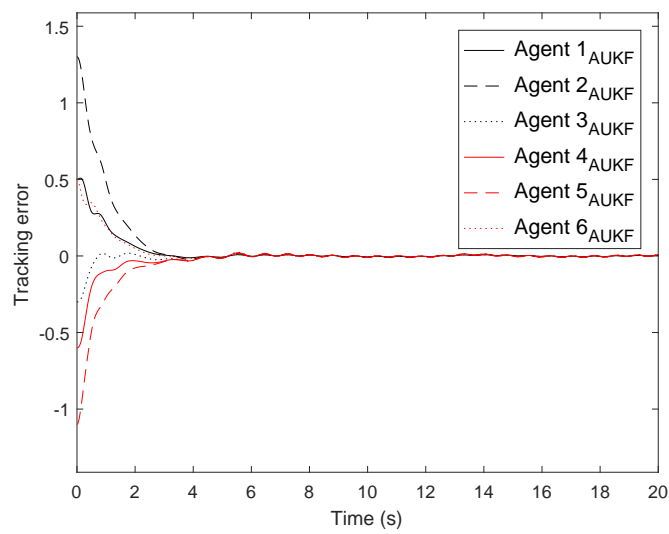

(c) $e_{\mathrm{x} i 1_{A U K F}}$

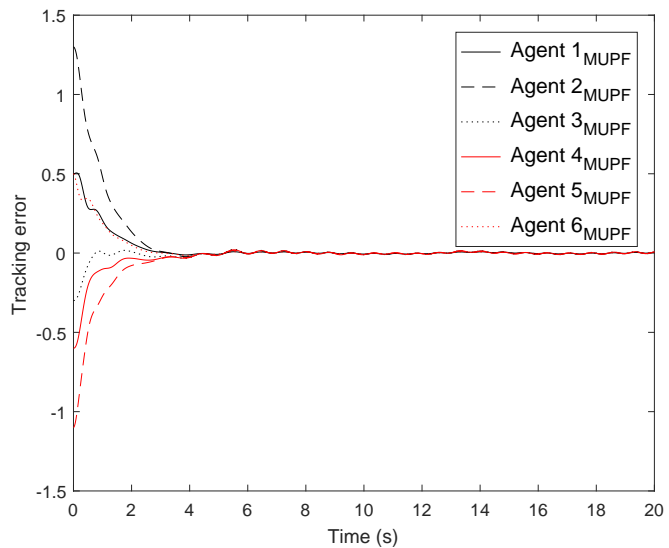

(e) $e_{\mathrm{x} i 1_{M U P F}}$

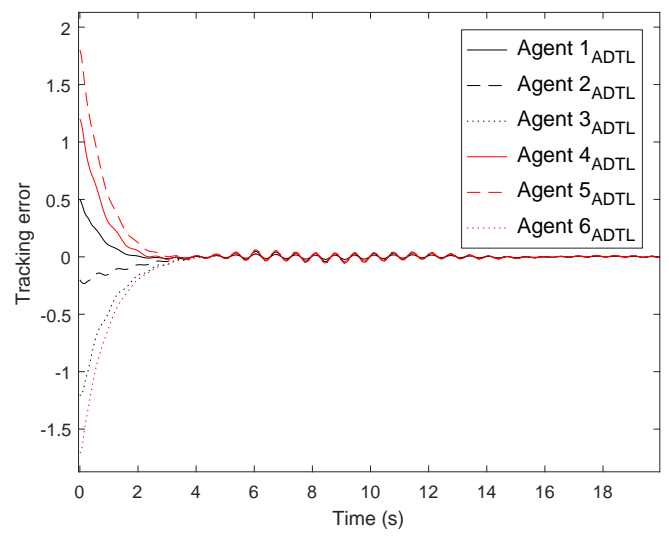

(b) $e_{\mathrm{x} i 2_{A D T L}}$

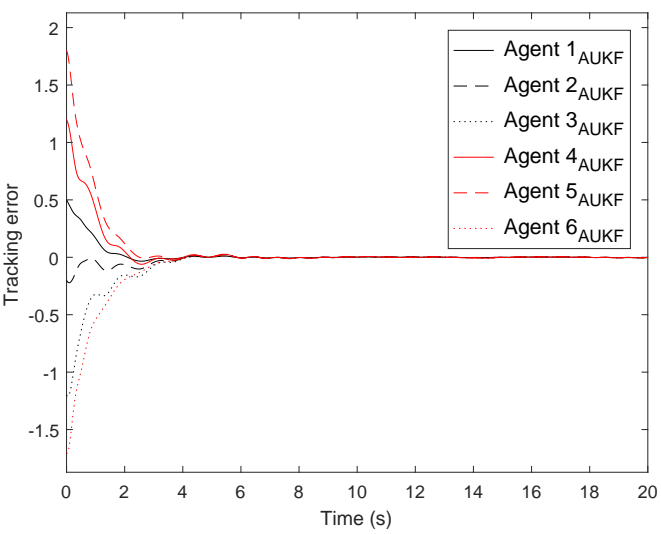

(d) $e_{\mathrm{x} i 2_{A U K F}}$

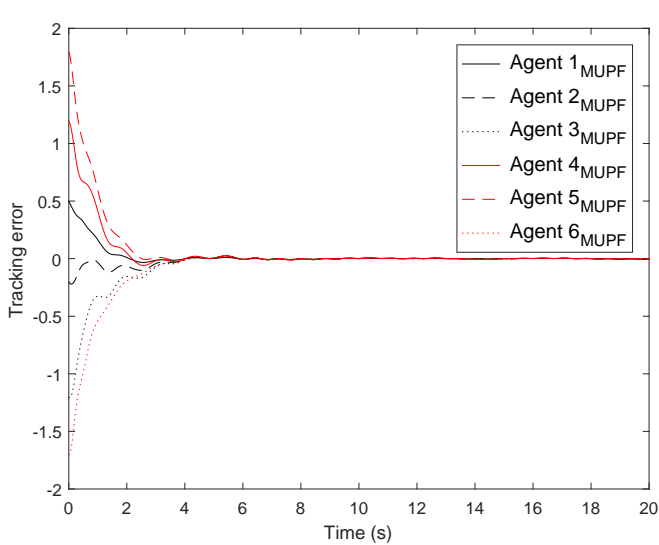

(f) $e_{\mathrm{x} i 2_{M U P F}}$

Figure 5.19: Tracking error under a constant control command $e_{i}=\left(e_{\mathrm{x} i 1}, e_{\mathrm{x} i 2}\right)^{T}(i=$ $1,2, \cdots, 6)$. 


\section{Chapter 5. Online Estimation and Control of Multi-Agent Systems}
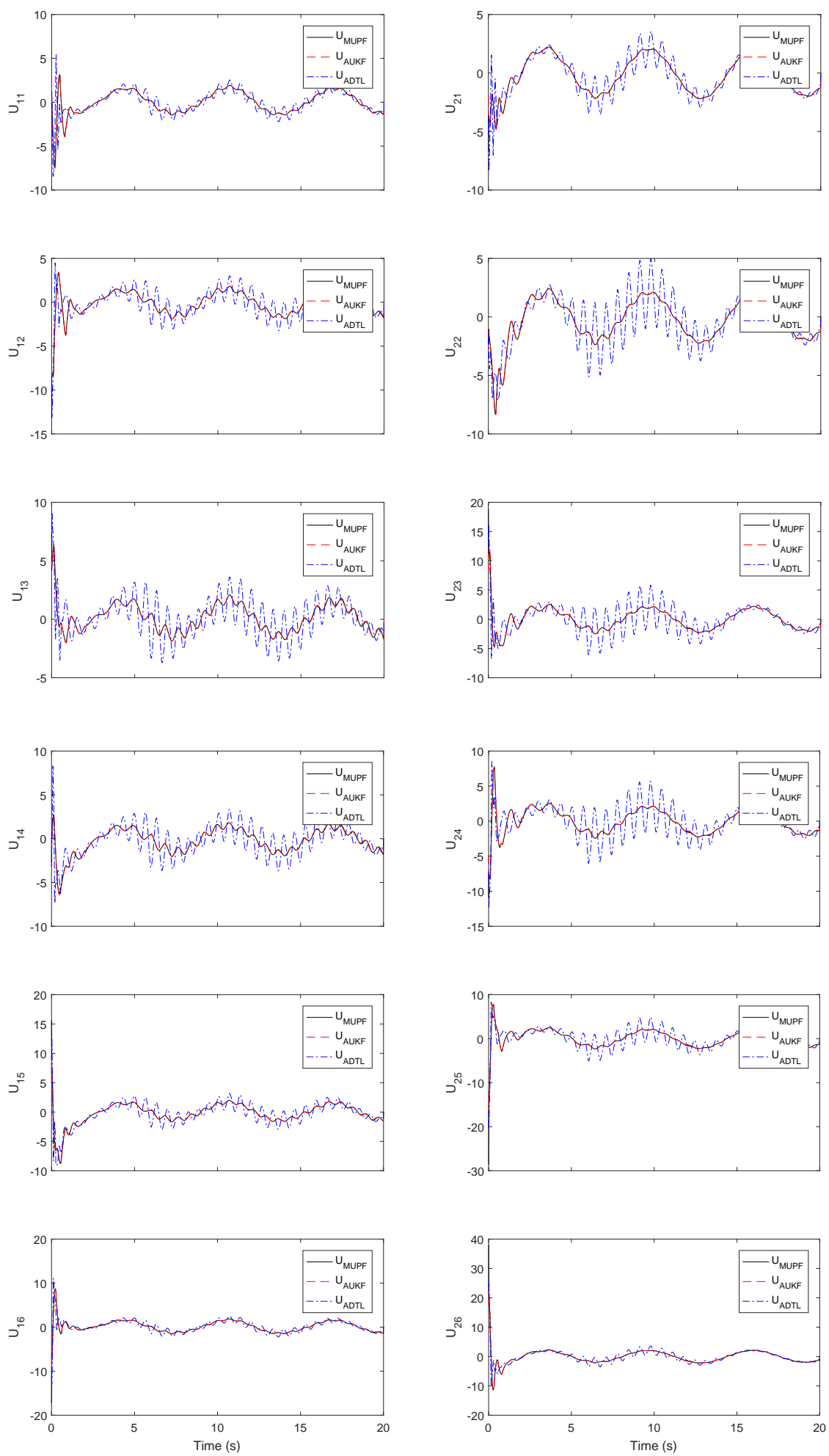

Figure 5.20: Control input under a constant control command $u_{i}=\left(u_{i 1}, u_{i 2}\right)^{T}(i=$ $1,2, \cdots, 6)$. 


\subsection{Simulation Results and Discussion}

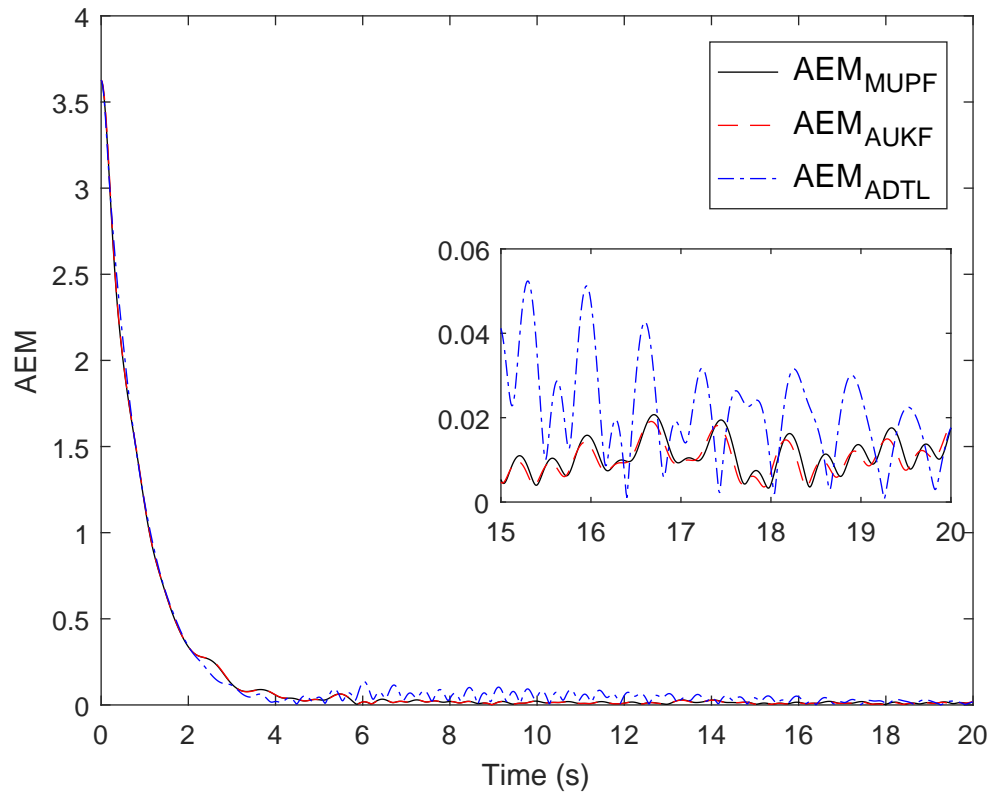

(a) AEM

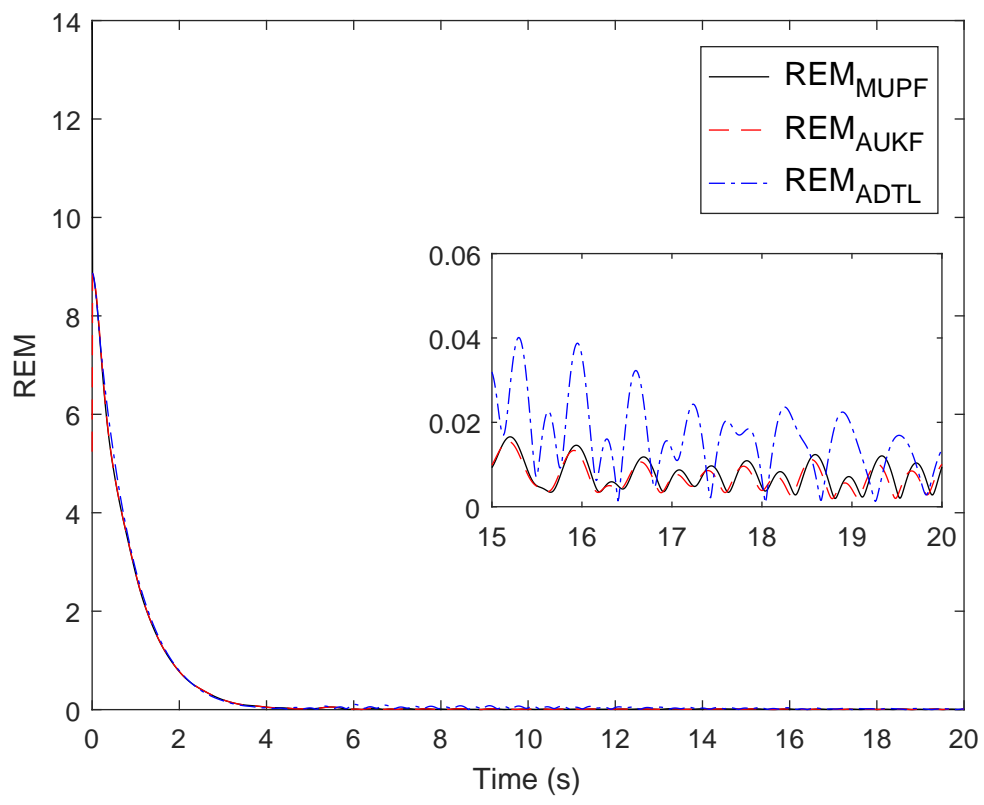

(b) REM

Figure 5.21: Absolute error metric and relative error metric under a constant control command. 


\section{Chapter 5. Online Estimation and Control of Multi-Agent Systems}

\subsection{Conclusions}

In this chapter, nonlinear state and parameter estimation techniques were investigated with the purpose of estimating an uncertain dynamic model which describes the evolution of a distributed multi-agent system in consensus tracking. A nonlinear filter was developed which is capable of joint estimation in an online setting. The proposed filter uses the RaoBlackwellised principle to couple the particle filtering technique with unscented transform principle. The new filter is called a marginalized unscented particle filter (MUPF). A Chebyshev neural network (CNN) was incorporated to describe the uncertain dynamic models of the multi-agent system while nonlinear filtering policies were implemented to estimate the state of the multi-agent system and to train the internal parameters of the neural network as neatly as possible given a set of prior measurements. The proposed filter was compared to the performance of the adaptive unscented Kalman filter and Adaptive Law under fault-free and fault-prone conditions at the multi-agent dynamics and control commands. 


\section{Chapter 6}

\section{Conclusions}

Nonlinear filtering involve estimation of the hidden state or parameters of interest given a sequence of measurements. Generally, the hidden state and parameters have an infinite set of possible values in its domain and the objective of filtering is to estimate the posterior distribution, i.e., an optimal estimate given evidence up to the current time. Computation of the required posterior distribution provide a complete solution of the estimation problem; however the computation involves complicated statistical procedures and it requires knowledge of the initial density, the transition density, and the likelihood function. Since most dynamic systems are inherently nonlinear, the application of nonlinear filtering is wide spread in several disciplines including communication, machine learning, system identification, finance, and many others. In this research, the primarily focus was directed to joint estimation using Bayesian algorithms with application in space systems. The work addresses a comparison between sub-optimal and non-optimal filters for applications in nonlinear and non-stationary dynamic processes. Throughout the thesis an attempt was made to redesign the non-optimal filters in such a way that under theoretical and well documented statistical procedures the filter will become sub-optimal. Few of these steps included the introduction of a properly selected importance sampling in particle weight calculations, introduction of a resampling (selection) procedure to reduce the degeneracy of the particles, reducing the resampling procedure by introduction of an effective sample size, reducing the variance of the particle filter by introduction of Rao-Blackwellised procedure etc. Throughout the work, a compared was made between the adaptive unscented Kalman filter (AUKF) and particle filter estimation techniques. The investigated filters were compared throughout the research and applied to the problems of Attitude estimation and control of monolithic spacecraft, distributed filtering for velocity-free attitude estimation of spacecraft formation flying, and, online estimation and control of multi-agent systems. 
Chapter 6. Conclusions

\subsection{Summary of Contributions}

Estimation of a nonlinear dynamic system is practically challenging and an open issue which arises since the hidden state of the dynamic model cannot be measured directly. Instead, a measurement model is designed to estimate the hidden state of the dynamic system as it evolves with time. The estimation process is then used to characterize the posterior distribution of the hidden state and parameter given the knowledge of the prior distribution and evidence from new measurements. The empirical evidence represents the sequence of measurements taken from the physical reality. In this research different filtering techniques have been applied to solve several estimations problems in space systems.

\subsubsection{Attitude estimation and control of monolithic spacecraft}

Online attitude estimation and control of monolithic spacecraft plays a major role in any space application and it requires to develop an accurate and stable online attitude estimation algorithm in order to increase the pointing capability of the spacecraft. This problem is well understood and many research has been published on the topic from different estimation and control perspectives.

Attitude estimation in a sequential setting was investigated with the presence of faults and unknown angular velocity measurements under quaternion constraints. Generally, online state estimation in attitude problems requires a sequential update of the attitude and angular velocity when new measurements become available. In this research, the attention was directed to (1) estimation of spacecraft attitude and angular velocity in a sequential setting and in the absence of angular velocity measurements, and (2) study the behavior of the sequential nonlinear filters in the presence of faults and uncertainties at the spacecraft dynamics, control commands, and sensor measurements. Two nonlinear filtering techniques were investigated to estimate the attitude and angular velocity, namely, adaptive unscented Kalman filter and particle filter. In particle filtering technique, the multinomial resampling algorithm was implemented to sample from the target distribution. The PF provided accurate attitude estimations since the particle representation of the posterior distribution are drawn at random rather than in a specific and deterministic manner as 


\subsection{Summary of Contributions}

in the AUKF. Generally, the AUKF generate a small number of samples from a Gaussian distribution and that may not represent adequately a complicated posterior distribution of the attitude which may include bias i.e. skewness, kurtosis of distribution or measurement outliers.

\subsubsection{Distributed attitude estimation and control of spacecraft for- mation flying}

Distributed spacecraft formation flying (SFF) is an active research topic that deal with maintaining a desired formation between spacecraft in a distributed setting. A major challenge of the problem is the requirement to estimate relative state (i.e., position and velocity) and relative attitude with respect to a local point of the spacecraft group. The state estimation of becomes a serious challenge in the presence of system faults and external disturbances which causes a drift in both relative positions and formation center of the spacecraft. An efficient and accurate distributed nonlinear filtering techniques was investigated with the purpose of estimating the unmeasurable angular velocity and reference angular acceleration of the SFF which is comprised of six spacecraft and a virtual leader which exchange partial information with other spacecraft in the formation. The modified Rodrigues parameters (MRP) are incorporated to describe the spacecraft attitude with respect to an inertial frame. Each spacecraft runs its own local filter to accurately approximate the posterior distribution of the unmeasurable angular velocity while a global distributed filter is incorporated to obtain a reference angular acceleration estimates of the virtual leader. The distributed filter is required since the virtual leader exchange only partial information with other spacecraft in formation. The adaptive unscented Kalman filter $(\mathrm{AUKF})$ and particle filter $(\mathrm{PF})$ techniques were investigated in parallel with a global distributed filter and an asymptotic output feedback control law to simultaneously track a common time-varying reference attitude while the reference attitude is available only to a subset of the spacecraft in the formation. A comparison is conducted between the particle filter and adaptive unscented Kalman filter in terms of the accuracy of estimating the unmeasurable angular velocity with the purpose of decreasing the station-keeping and formation-keeping attitude errors. 
Chapter 6. Conclusions

\subsubsection{Online estimation and control of multi-agent systems}

A multi-agent system distribute the task responsibilities among a number of individual agents, each operating with partial information of state estimates and response of other agents. When the dynamic model of the multi-agent system is not exact or partially known neural networks techniques can be used to estimate any smooth function to an arbitrary accuracy. Chebyshev neural network (CNN) was introduced to describe the uncertain dynamic models of the multi-agent system and nonlinear filtering techniques are implemented to estimate the state of the multi-agent system and to train the internal parameters of the neural network as neatly as possible given a set of prior measurements.

A nonlinear filter has been proposed such that the filter couples the particle filtering algorithm with Rao-Blackwellised technique and unscented transform principle and applied the proposed filter in online joint estimation of an uncertain dynamic models which describe the evolution of a distributed multi-agent system in consensus tracking. The new filter is called marginalized unscented particle filter (MUPF). The idea behind the proposed filter is to partition the dynamic state space into two separate subspaces such that the posterior distribution can be updated analytically and efficiently. One part of the decomposed state space is estimated using a sub-optimal adaptive unscented Kalman filter whereas the other is estimated using particle filtering technique. Since the first part is estimated with a suboptimal filter instead of a pure sampling approach, the computing power is saved and the variance is reduced. Most of the emphasis was placed on accuracy of the joint estimation process, as well as the capacity to estimate CNN and determine the uncertain dynamics of the system. The MUPF, AUKF and the adaptive law were investigated under fault-free and fault-prone conditions at the multi-agent dynamics and control commands. 


\subsection{Future Work}

\subsection{Future Work}

Nonlinear filtering techniques have become one of the standard tools in estimation theory which allow the Bayesian paradigm to be routinely applied to sophisticated nonlinear dynamic models. As the understanding of nonlinear filtering techniques increase and technology is available to deal with the computational demand of these algorithm, better estimations and posteriori distributions could be expected. In addition, we may expected to deal with high dimensionality of complex nonlinear dynamic models where both state and parameters are estimated simultaneously. There are still many issues with implementing nonlinear filters which provide consistent, efficient, robust, with minimal variance of estimations. The future direction of this research include implementation of different proposal distributions and testing new resampling strategies for sequential Monte Carlo filtering technique with the general idea of improving nonlinear filtering techniques.

\subsubsection{Proposal distributions}

The sequential importance sapling (SIS) algorithm includes an importance sampling which help to sample the distribution in the region of importance in order to achieve the computational efficiently. Selecting an effective proposal distribution still lacks rigorous theoretical justification and essentially selecting a proposal distribution becomes a design problem. Therefore, selecting an appropriate proposal distribution is a key in to apply a successful importance sampling. Generally, the optimal proposal distribution is a distribution that minimizes the conditional variance given the latest measurement. In the scientific literature, many proposal distributions were reported including prior distribution, likelihood distribution, EKF as proposal distribution and unscented Kalman filter. The idea behind unscented Kalman filter (UPF) [van der Merwe et al. 2000], [Wan \& Van Der Merwe 2000] is to use Gaussian approximation for the proposal distribution of the SIS algorithm. This result in a filter that perform better since the rate at which the tails of the proposal distribution reduce to zero can be controlled. This research can be extended by identifying better proposal distribution which will reduce the computational efficiently of the algorithm. 
Chapter 6. Conclusions

\subsubsection{Resampling strategies}

The resampling step avoids the degeneracy problem in sequential importance sampling (SIS) algorithm where after few iterations all but one particle have a negligible weight. The resampling step replicate particles with large weights and remove the ones with negligible weight. The scientific literature on resampling strategies identifies many approached for resampling in particle filtering setting. Few of the strategies include multinomial, residual, stratified and systematic resampling, residual resampling, local Monte Carlo resampling, stratified sampling, Gibbs sampling etc. In this research primarily focus was placed on multinomial resampling and systematic resampling. The extension to this research will focus on proposing and testing different resampling strategies in order to select important samples in a more efficient way.

\subsubsection{Improved nonlinear filtering techniques}

There are many unanswered question in nonlinear filtering theory especially when it comes to joint estimation in high-dimensional spaces. For example, how to select a prior distribution in order to compute the required posterior distribution which minimize the variance? This is indeed an unsolved issue in nonlinear filtering and control problems. Another question relies on the Principle of Total Evidence. In general, the filtering process should follow the Principle of Total Evidence where all relevant measurements should be utilized for computing an updated posterior distribution. Collecting measurements is essential for computing the posterior distribution which minimize the mean squared error of estimation. From a Bayesian perspective this is an important aspect and new measurements have to be incorporated once a measurement from the physical world becomes available. The question is, how many measurements are needed to generate an efficient posterior distribution? There is no immediate answer to these questions but the idea is to propose an "optimal" nonlinear filter which satisfy the following properties:

- Consistency: The filter is consistent if the posterior distribution converges to the true state almost surly as the number of measurements approaches infinity.

- Efficiency: The filter is efficient if it produces the smallest error covariance matrix 


\subsection{Concluding Remarks}

among all unbiased filter.

- Minimum variance: Variance reduction is the central issue of various Monte Carlo approximation methods, most of the proposed improvements are geared towards variance-reduction, i.e., selecting proposal distribution and resampling strategies.

- Robustness: The filter is robust if it is insensitive to the gross measurement errors and the uncertainties in measurements.

- Unbiasedness: An filter is unbiased if its posterior distribution is equal to the true state or parameter of interest.

\subsection{Concluding Remarks}

Nonlinear filtering techniques provide a powerful means for estimating the hidden state of a dynamic systems given the trajectory of measurements. In this dissertation an investigation of different perspectives of nonlinear filtering and proposed a nonlinear filter for joint estimation. The primarily emphasis was the application in space systems where the dynamic equations are highly nonlinear. I hope this dissertation provided a greater exposure to the field of space systems and nonlinear filtering techniques, and constitute a valuable source of information of various nonlinear filtering techniques which are applicable in the aerospace industry. 


\section{Appendices}





\section{APPENDIX A}

\section{Importance Sampling}

Typically, at any time $t$, it is impossible to sample efficiently from the target posterior distribution $p\left(\mathrm{x}_{0: t} \mid y_{1: t}\right)$. Instead, we estimate $p\left(\mathrm{x}_{0: t} \mid y_{1: t}\right)$ using the well know importance sampling method [Halton 1962]. The basic idea is simple and it is based on the following observations. Let us introduce an arbitrary importance distribution $q\left(\mathrm{x}_{0: t} \mid y_{1: t}\right)$, from which it is easy to get samples, and suppose that $p\left(\mathrm{x}_{0: t} \mid y_{1: t}\right) \propto q\left(\mathrm{x}_{0: t} \mid y_{1: t}\right)$. In addition, let $\mathrm{x}^{(i)} \sim q(\mathrm{x}) i=1, \ldots N_{s}$ be samples that are easily generated from a proposal $q(\cdot)$ called the importance density ${ }^{1}$. Then, a weighted approximation to the target density is given by

$$
p\left(\mathrm{x}_{0: t} \mid y_{1: t}\right) \approx \sum_{i=1}^{N_{s}} \tilde{w}_{0: t}^{(i)} \delta\left(\mathrm{x}_{0: t}-\mathrm{x}_{0: t}^{(i)}\right)
$$

where the weight

$$
\tilde{w}_{0: t}^{(i)} \propto \frac{p\left(\mathrm{x}_{0: t} \mid y_{1: t}\right)}{q\left(\mathrm{x}_{0: t} \mid y_{1: t}\right)}
$$

is a normalized weight of the $i$ th particle where the samples $\mathrm{x}_{0: t}^{(i)}$ were drown from importance density $q\left(\mathrm{x}_{0: t} \mid y_{1: t}\right)$. In a sequential setting, approximation of the posterior distribution $p\left(\mathrm{x}_{0: t} \mid y_{1: t}\right)$ at each iteration is possible given samples constituting an approximation to $p\left(\mathrm{x}_{0: t-1} \mid y_{1: t-1}\right)$. If the importance density is chosen to factorize such that

$$
q\left(\mathrm{x}_{0: t} \mid y_{1: t}\right)=q\left(\mathrm{x}_{t} \mid \mathrm{x}_{0: t-1}, y_{1: t}\right) q\left(\mathrm{x}_{0: t-1} \mid y_{1: t-1}\right)
$$

then one can obtain samples $\mathrm{x}_{0: t}^{(i)} \sim q\left(\mathrm{x}_{0: t} \mid y_{1: t}\right)$ by augmenting each of the existing samples $\mathrm{x}_{0: t-1}^{(i)} \sim q\left(\mathrm{x}_{0: t-1} \mid y_{1: t-1}\right)$ with the new samples $\mathrm{x}_{t}^{(i)} \sim q\left(\mathrm{x}_{t} \mid \mathrm{x}_{0: t-1}, y_{1: t-1}\right)$.

\footnotetext{
${ }^{1}$ The notation $\mathrm{x}^{(i)} \sim q(\mathrm{x})$ means that the state hypothesis $\mathrm{x}^{(i)}$ is "distributed according to" importance density $q(\mathrm{x})$ for $i=0, \ldots, N_{p}$.
} 


\section{APPENDIX B}

\section{Rao-Blackwellisation}

The underlying principle of RBPF is the partition of a dynamic state space $\mathbf{z}_{0: t}$ into two subspaces $\mathbf{x}_{0: t}$ and $\mathbf{r}_{0: t}$, such that the distribution $p\left(\mathbf{r}_{0: t}, \mathbf{x}_{0: t} \mid y_{1: t}\right)$ can be updated analytically and efficiently. Here the dynamic state space is decomposed into two parts, one part is being calculated exactly using Kalman filters whereas the other is estimated using particle filter. Since the first part is estimated exactly, the computing power is saved and the variance is reduced. If we were able to sample $N$ i.i.d. random samples $\left(\mathbf{r}_{0: t}^{(i)}, \mathbf{x}_{0: t}^{(i)}\right) i=1, \ldots, N$, according to $p\left(\mathbf{r}_{0: t}, \mathbf{x}_{0: t} \mid y_{1: t}\right)$, then an empirical estimate of this distribution would be given by [Doucet et al. 2000]

$$
\hat{p}_{N}\left(\mathbf{r}_{0: t}, \mathbf{x}_{0: t} \mid y_{1: t}\right)=\frac{1}{N} \sum_{i=1}^{N} \delta_{\left(\mathbf{r}_{0: t}^{(i)}, \mathbf{x}_{0: t}^{(i)}\right)}\left(d \mathbf{r}_{0: t}, d \mathbf{x}_{0: t}\right)
$$

where $\delta_{\left(\mathbf{r}_{0: t}^{(i)}, \mathbf{x}_{0: t}^{(i)}\right)}$ denotes the Direc delta function located at $\left(\mathbf{r}_{0: t}^{(i)}, \mathbf{x}_{0: t}^{(i)}\right)$. It is easy to estimate the expected value of any function $\mathbf{f}_{t}$ of the hidden variable with respect to this distribution, $\mathbb{E}\left(\mathbf{f}_{t}\right)$, using

$$
\begin{aligned}
\overline{\mathbb{E}}\left(\mathbf{f}_{t}\right) & =\int \mathbf{f}_{t}\left(\mathbf{r}_{0: t}, \mathbf{x}_{0: t}\right) \hat{p}_{N}\left(\mathbf{r}_{0: t}, \mathbf{x}_{0: t} \mid y_{1: t}\right) d \mathbf{r}_{0: t}, d \mathbf{x}_{0: t} \\
& =\frac{1}{N} \sum_{i=1}^{N} \mathbf{f}_{t}\left(\mathbf{r}_{0: t}^{(i)}, \mathbf{x}_{0: t}^{(i)}\right)
\end{aligned}
$$

This estimate is unbiased and from the law of large numbers, $\overline{\mathbb{E}}\left(\mathbf{f}_{t}\right)$ converges almost surly towards $\mathbb{E}\left(\mathbf{f}_{t}\right)$ as $N \rightarrow \infty$. Typically, it is impossible to sample efficiently from the posterior distribution $p\left(\mathbf{r}_{0: t}, \mathbf{x}_{0: t} \mid y_{1: t}\right)$ an any time $t$ so we focus on alternative methods.

One way to estimate $p\left(\mathbf{r}_{0: t}, \mathbf{x}_{0: t} \mid y_{1: t}\right)$ and $\mathbb{E}\left(\mathbf{f}_{t}\right)$ consists of using the well-known importance sampling method. Lets introduce an arbitrary distribution $q\left(\mathbf{r}_{0: t}, \mathbf{x}_{0: t} \mid y_{1: t}\right)$, from 
which it is easy to sample then,

$$
\mathbb{E}\left(\mathbf{f}_{t}\right)=\frac{\mathbb{E}_{q\left(\mathbf{r}_{0: t}, \mathbf{x}_{0: t} \mid y_{1: t}\right)}\left(\mathbf{f}_{t}\left(\mathbf{r}_{0: t}, \mathbf{x}_{0: t}\right) w\left(\mathbf{r}_{0: t}, \mathbf{x}_{0: t}\right)\right)}{\mathbb{E}_{q\left(\mathbf{r}_{0: t}, \mathbf{x}_{0: t} \mid y_{1: t}\right)}\left(w\left(\mathbf{r}_{0: t}, \mathbf{x}_{0: t}\right)\right)}
$$

where the importance weight is equal to

$$
w\left(\mathbf{r}_{0: t}, \mathbf{x}_{0: t}\right)=\frac{p\left(\mathbf{r}_{0: t}, \mathbf{x}_{0: t} \mid y_{1: t}\right)}{q\left(\mathbf{r}_{0: t}, \mathbf{x}_{0: t} \mid y_{1: t}\right)}
$$

Given $N$ i.i.d. samples $\left(\mathbf{r}_{0: t}^{(i)}, \mathbf{x}_{0: t}^{(i)}\right) i=1, \ldots, N$ distributed according to $q\left(\mathbf{r}_{0: t}, \mathbf{x}_{0: t} \mid y_{1: t}\right)$, a perfect Monte Carlo estimate of $\mathbb{E}\left(\mathbf{f}_{t}\right)$ is given by

$$
\begin{aligned}
\overline{\mathbb{E}}\left(\mathbf{f}_{t}\right) & =\frac{\sum_{i=1}^{N} \mathbf{f}_{t}\left(\mathbf{r}_{0: t}^{(i)}, \mathbf{x}_{0: t}^{(i)}\right) w\left(\mathbf{r}_{0: t}^{(i)}, \mathbf{x}_{0: t}^{(i)}\right)}{\sum_{i=1}^{N} w\left(\mathbf{r}_{0: t}^{(i)}, \mathbf{x}_{0: t}^{(i)}\right)} \\
& =\sum_{i=1}^{N} \tilde{w}_{0: t}^{(i)} \mathbf{f}_{t}\left(\mathbf{r}_{0: t}^{(i)}, \mathbf{x}_{0: t}^{(i)}\right)
\end{aligned}
$$

where the normalizing importance weights $\tilde{w}_{0: t}^{(i)}$ are equal to

$$
\tilde{w}_{0: t}^{(i)}=\frac{w\left(\mathbf{r}_{0: t}^{(i)}, \mathbf{x}_{0: t}^{(i)}\right)}{\sum_{i=1}^{N} w\left(\mathbf{r}_{0: t}^{(i)}, \mathbf{x}_{0: t}^{(i)}\right)}
$$

This method is equivalent to the following point mass approximation of $p\left(\mathbf{r}_{0: t}, \mathbf{x}_{0: t} \mid y_{1: t}\right)$

$$
\bar{p}\left(\mathbf{r}_{0: t}, \mathbf{x}_{0: t} \mid y_{1: t}\right)=\sum_{i=1}^{N} \tilde{w}_{0: t}^{(i)} \delta_{\left(\mathbf{r}_{0: t}^{(i)}, \mathbf{x}_{0: t}^{(i)}\right)}\left(d \mathbf{r}_{0: t}, d \mathbf{x}_{0: t}\right)
$$


APPENDIX C

\section{Parameter Estimation of Chebyshev Neural Network}

In this section, we present the parameter of the hidden layer of a third-order Chebyshev Neural Network (CNN). The parameters were computed using the adaptive unscented Kalman filter (AUKF) and a marginalized unscented particle filter (MUPF). The parameters were calculated for various fault free and fault prone scenarios. 
Appendix C. Parameter Estimation of Chebyshev Neural Network

\section{C.1 Parameter estimation under fault-free scenario}
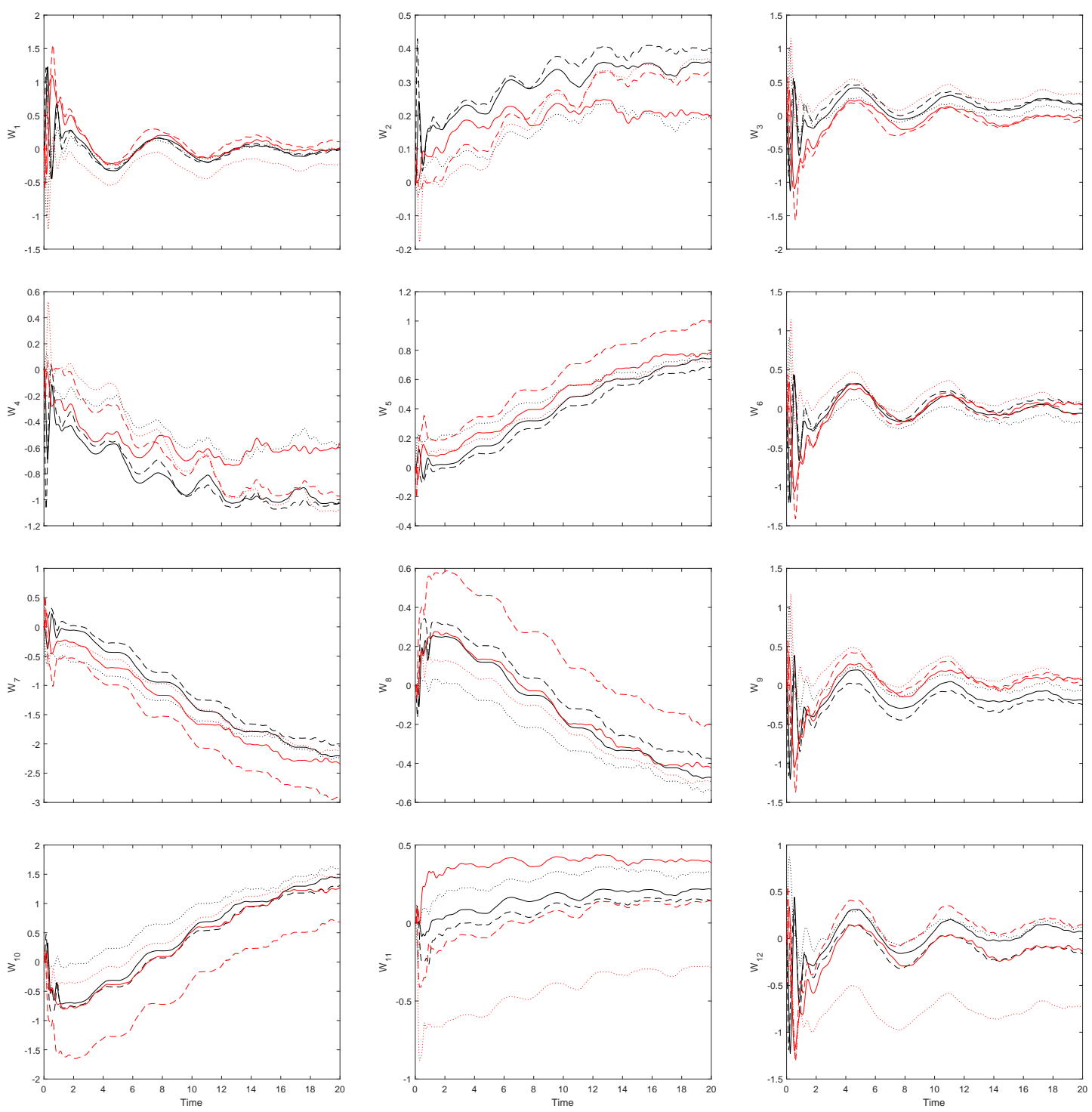

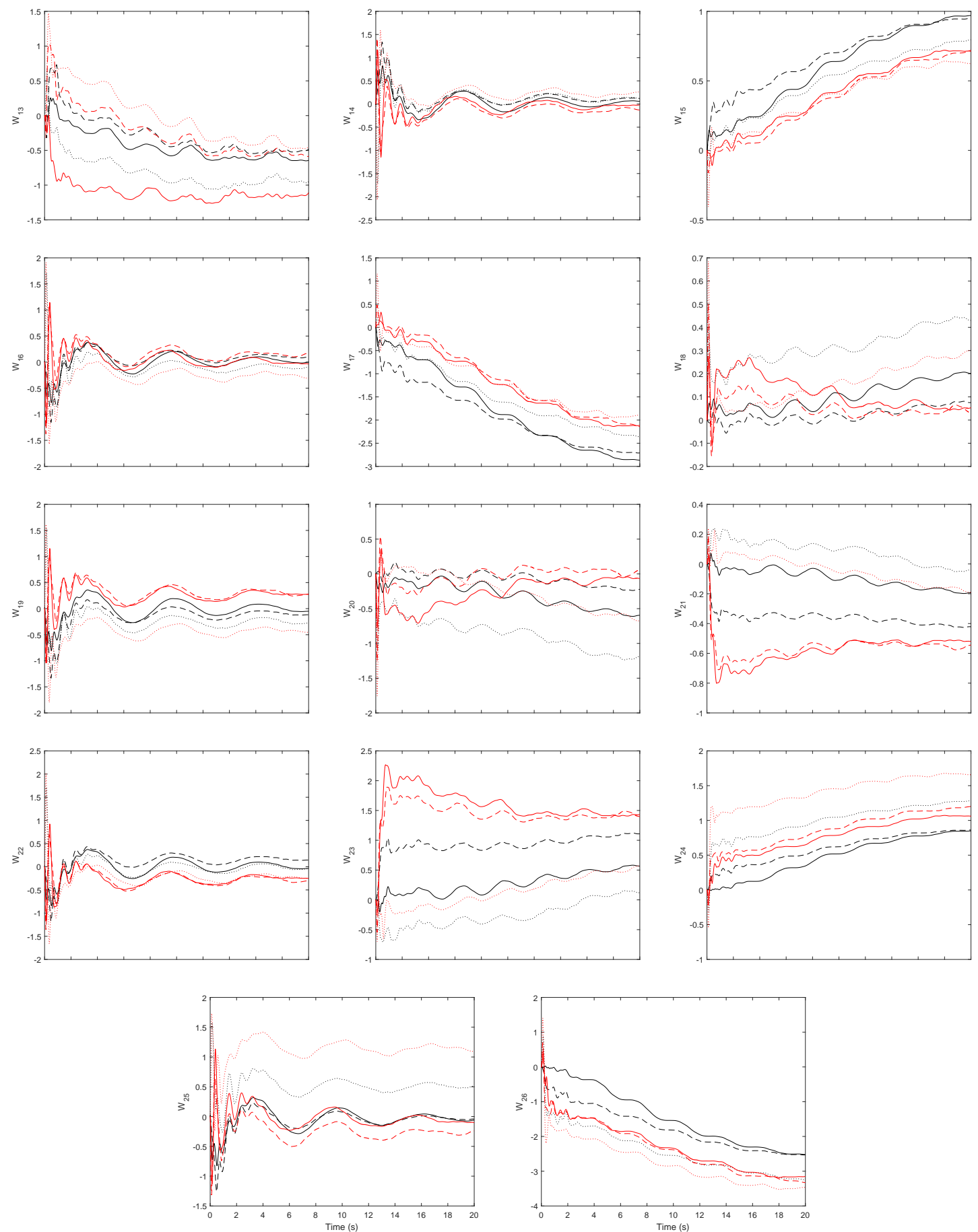

Figure C.1.1: Parameter estimation with adaptive unscented Kalman filter. 
Appendix C. Parameter Estimation of Chebyshev Neural Network
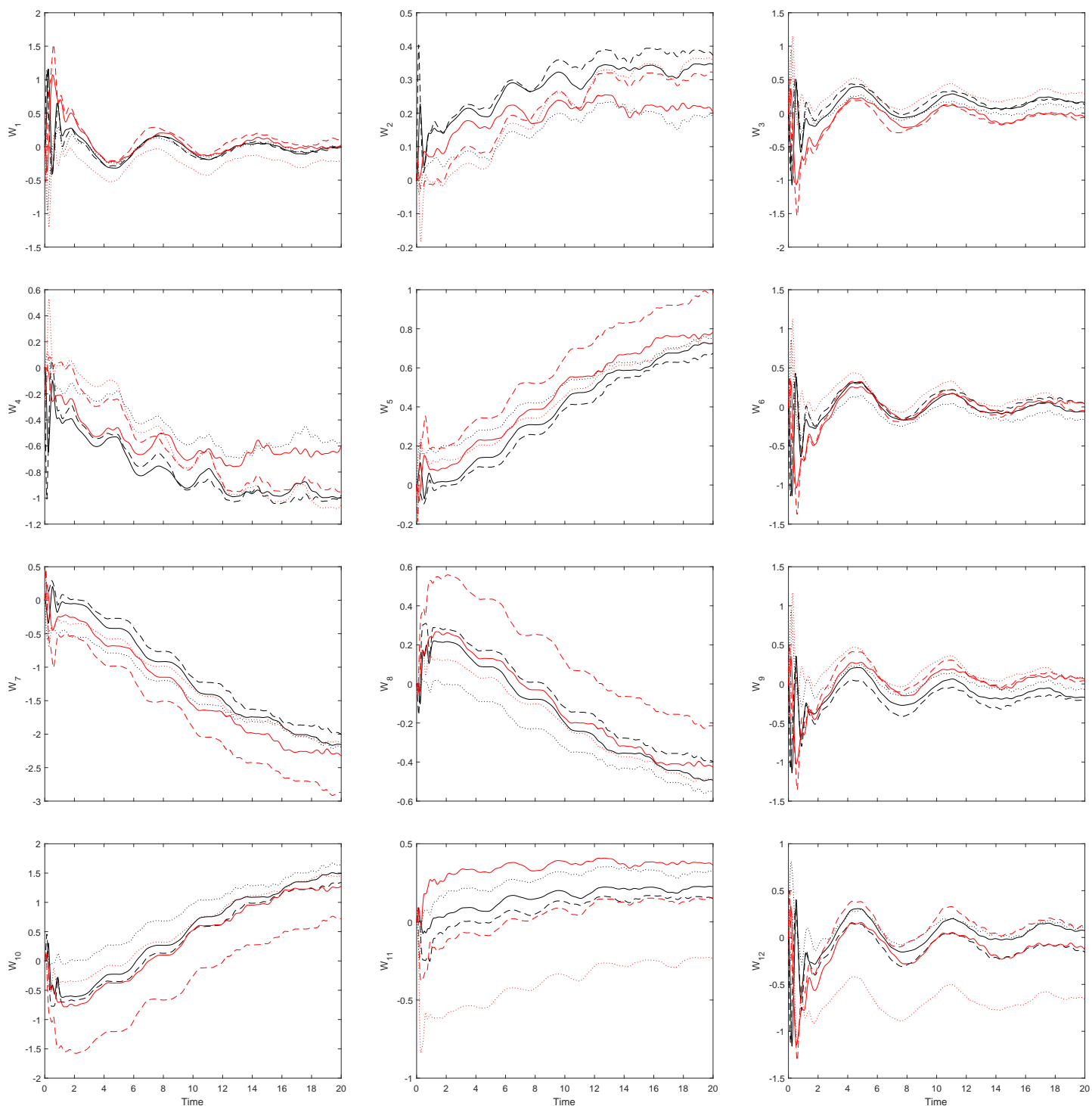

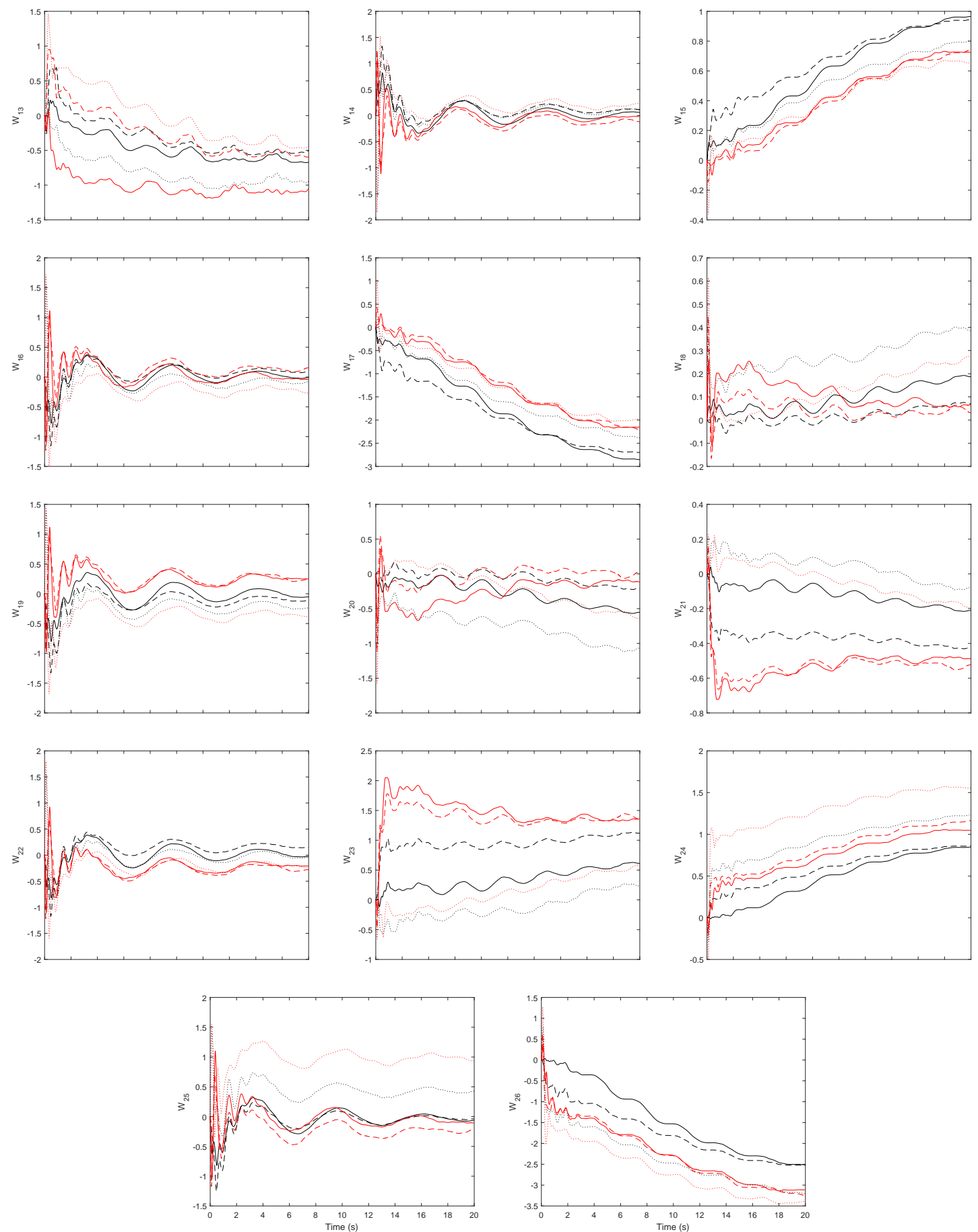

Figure C.1.2: Parameter estimation with marginalized unscented particle filter. 
Appendix C. Parameter Estimation of Chebyshev Neural Network

\section{C.2 Parameter estimation under uncertainty in dynam- ics model}
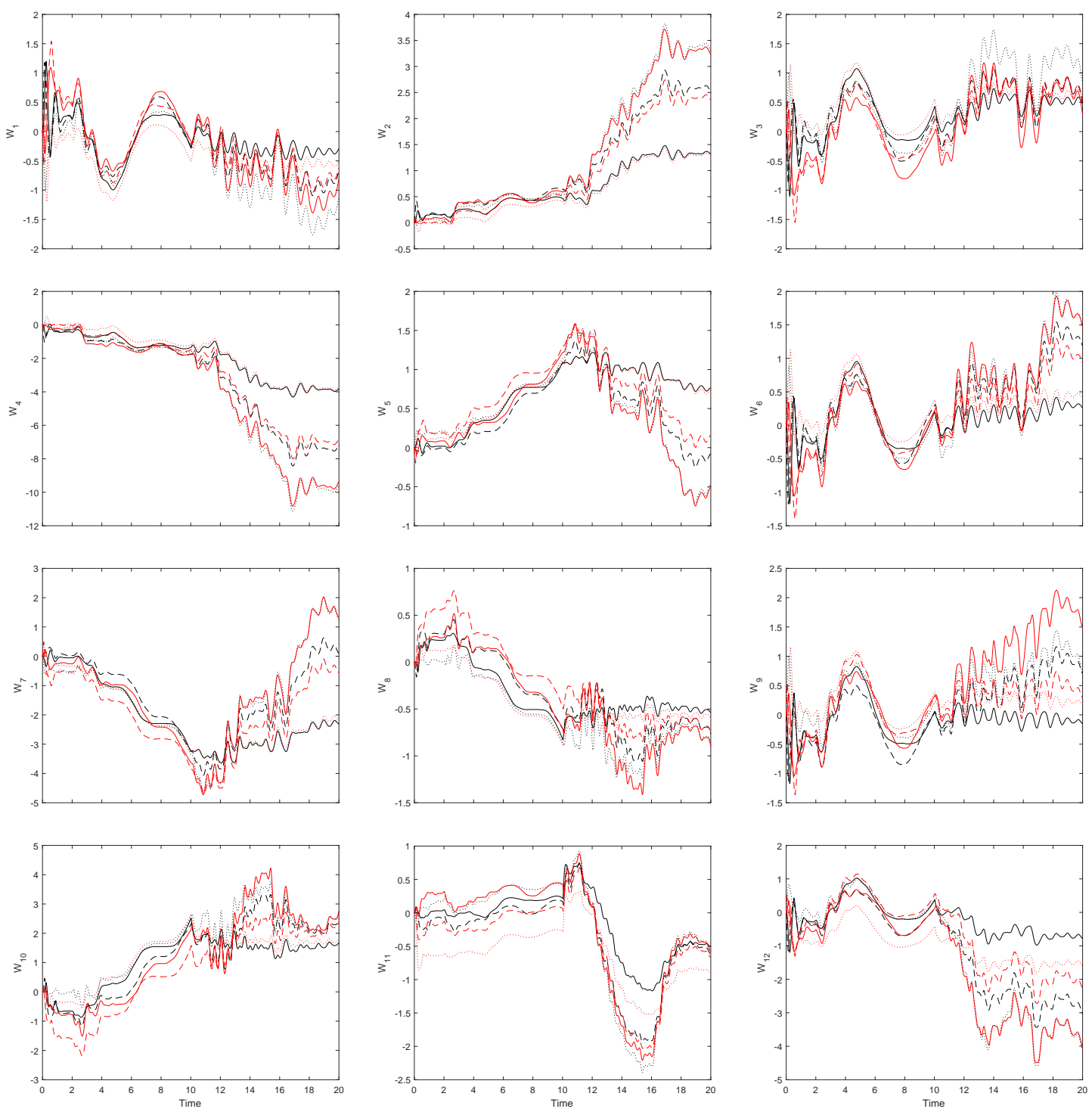
C.2. Parameter estimation under uncertainty in dynamics model
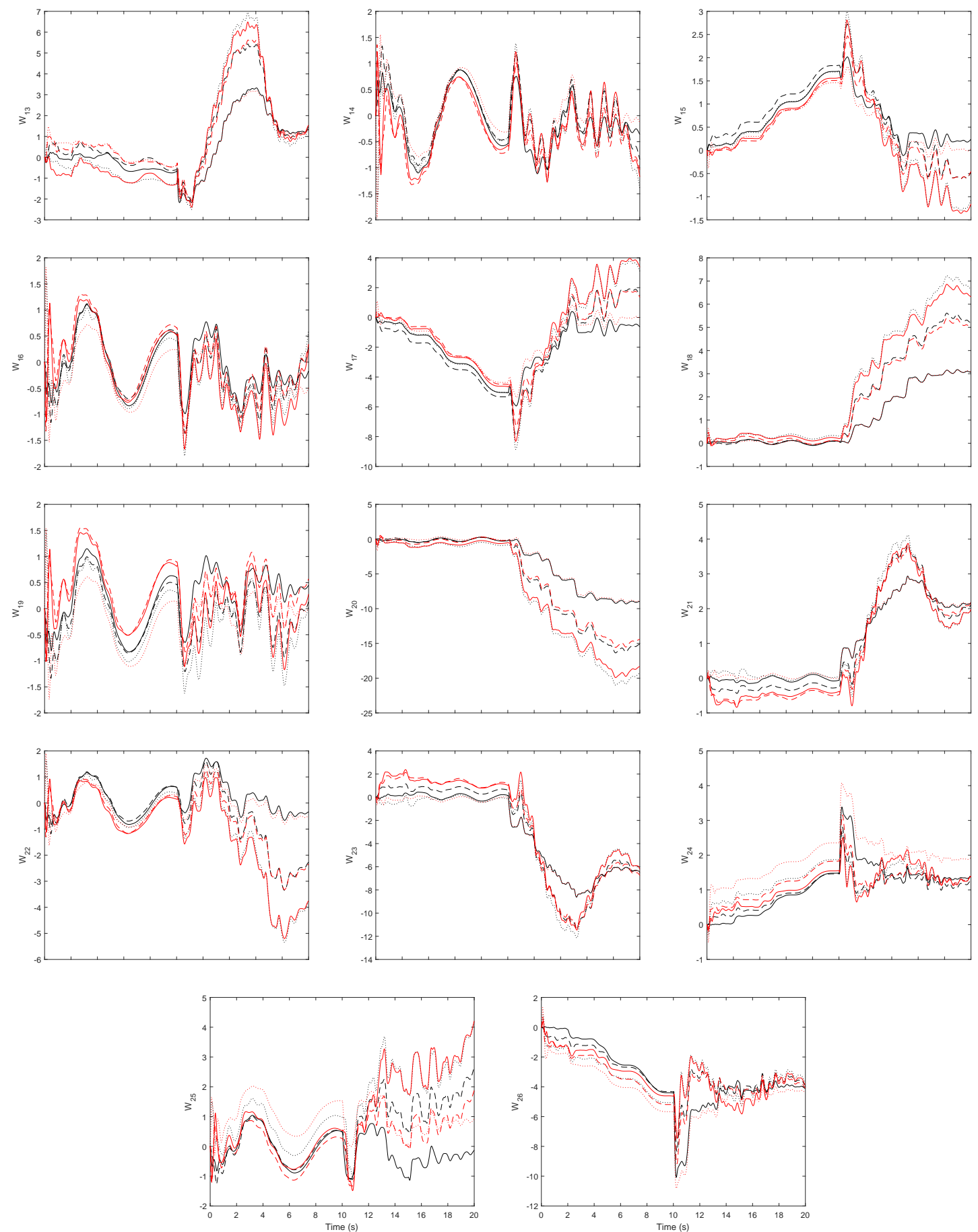

Figure C.2.1: Parameter estimation under uncertainty of dynamic model using adaptive unscented Kalman filter. 
Appendix C. Parameter Estimation of Chebyshev Neural Network
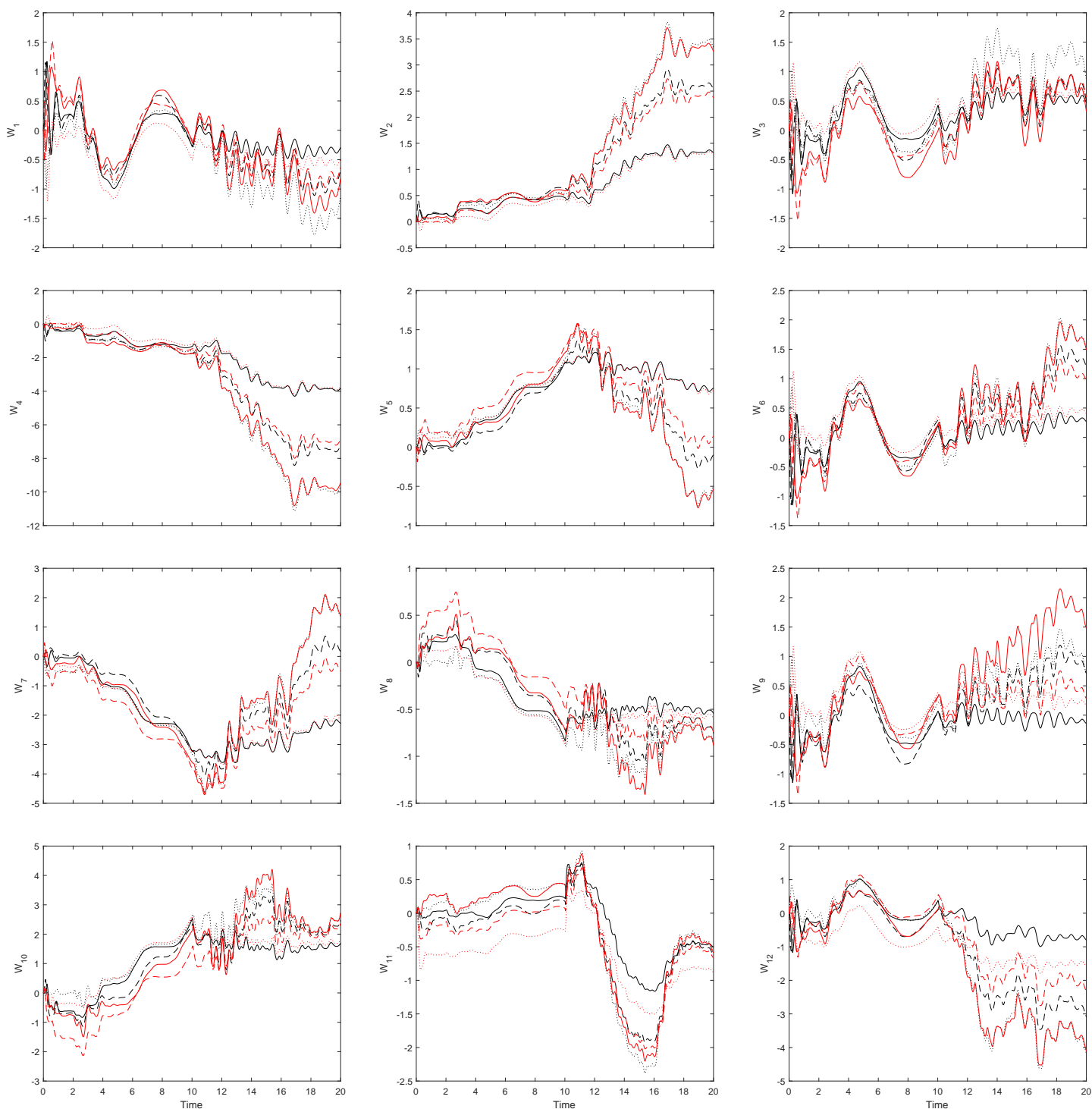
C.2. Parameter estimation under uncertainty in dynamics model
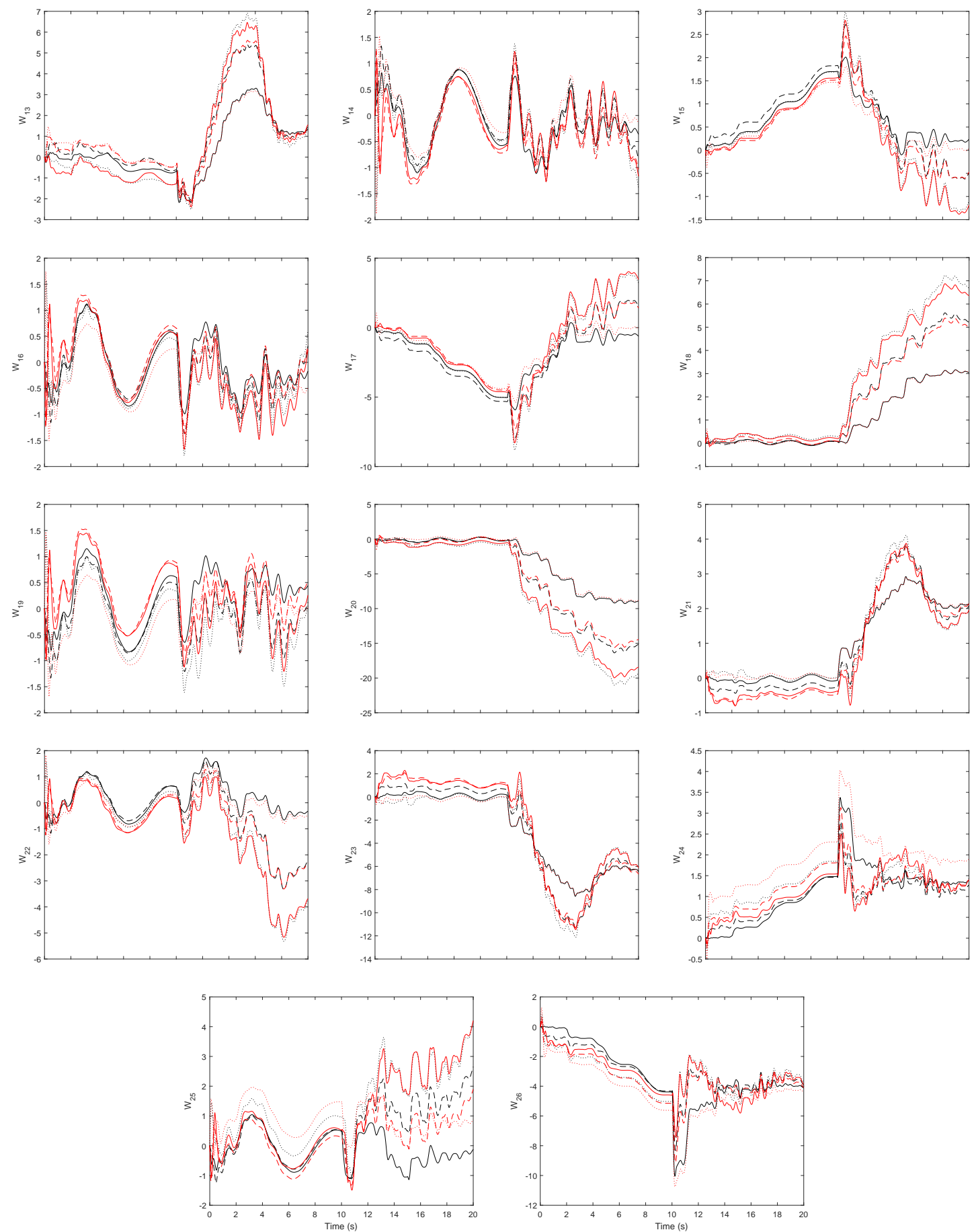

Figure C.2.2: Parameter estimation under uncertainty of dynamic model using marginalized unscented particle filter. 
Appendix C. Parameter Estimation of Chebyshev Neural Network

\section{C.3 Parameter estimation under external disturbances}
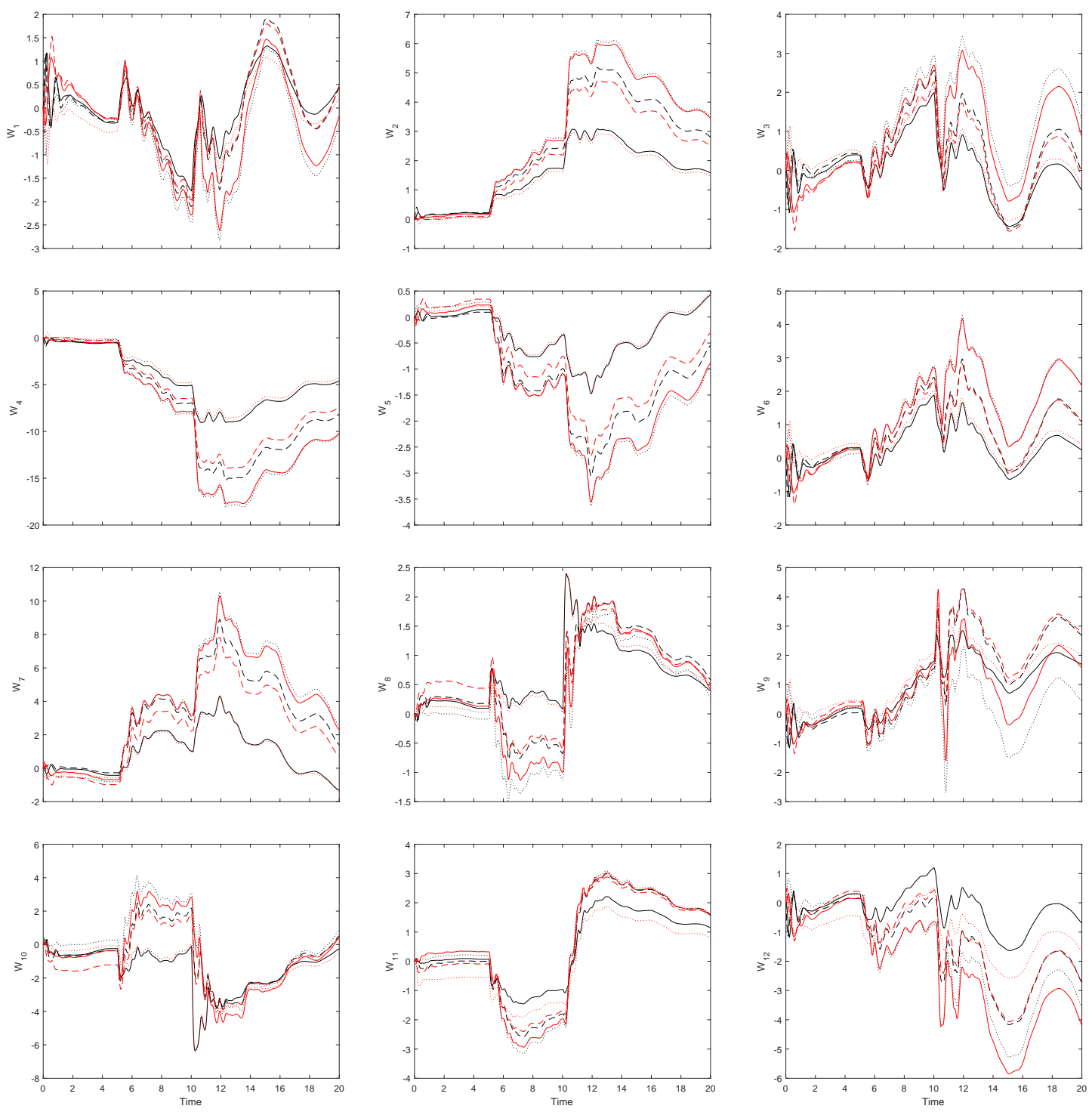


\section{C.3. Parameter estimation under external disturbances}
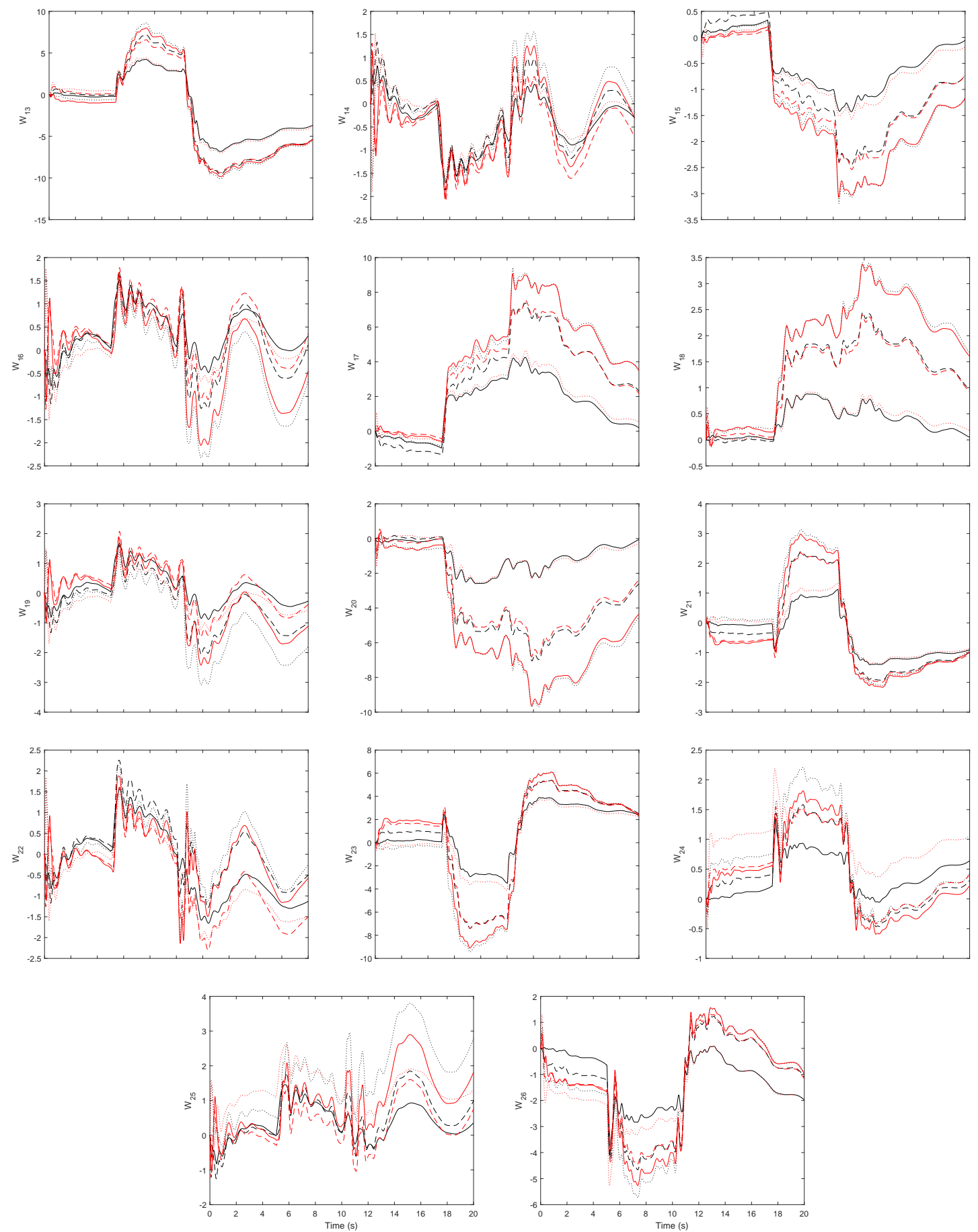

Figure C.3.1: Parameter estimation under external disturbances using adaptive unscented Kalman filter. 


\section{Appendix C. Parameter Estimation of Chebyshev Neural Network}
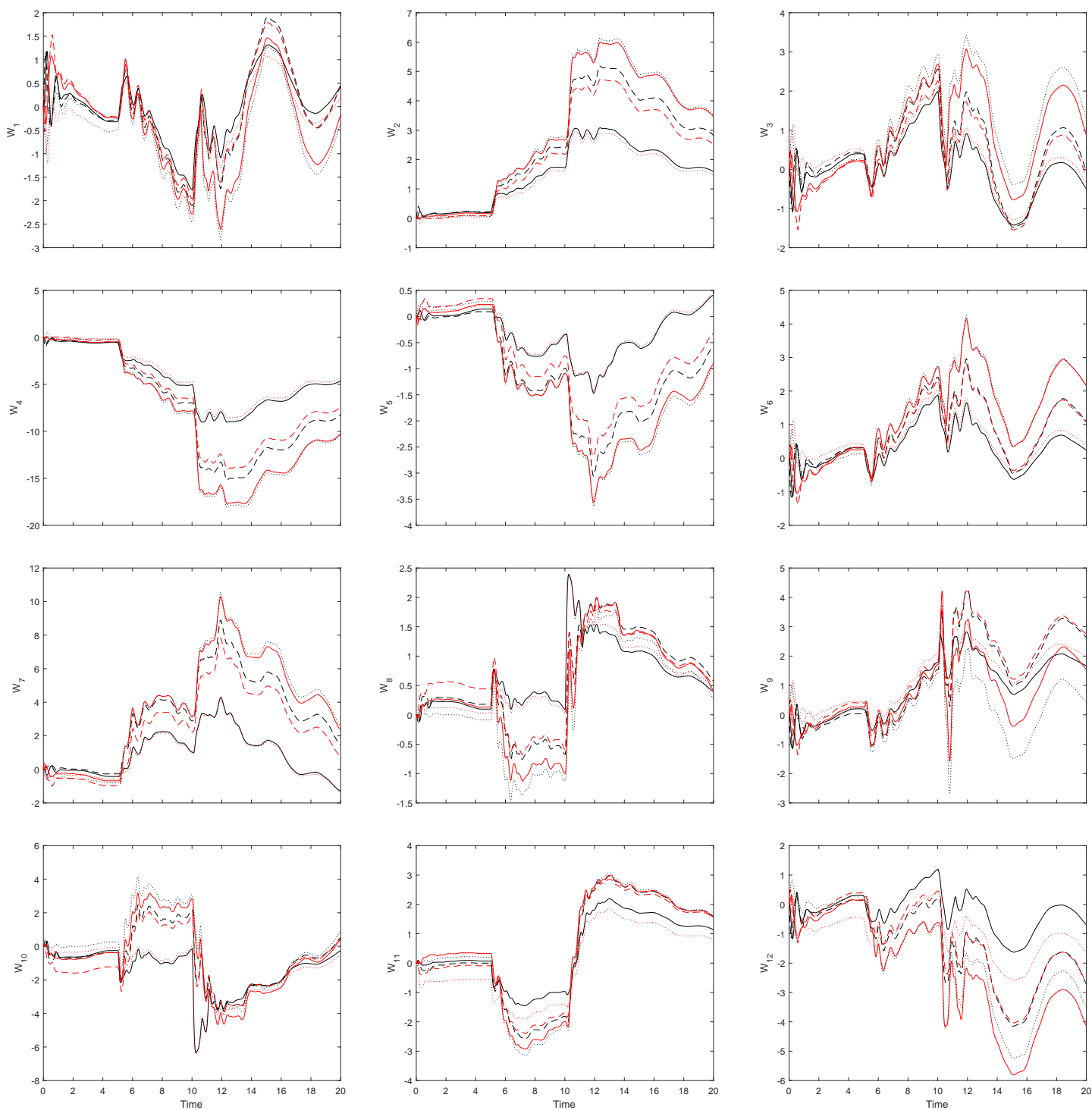


\section{C.3. Parameter estimation under external disturbances}
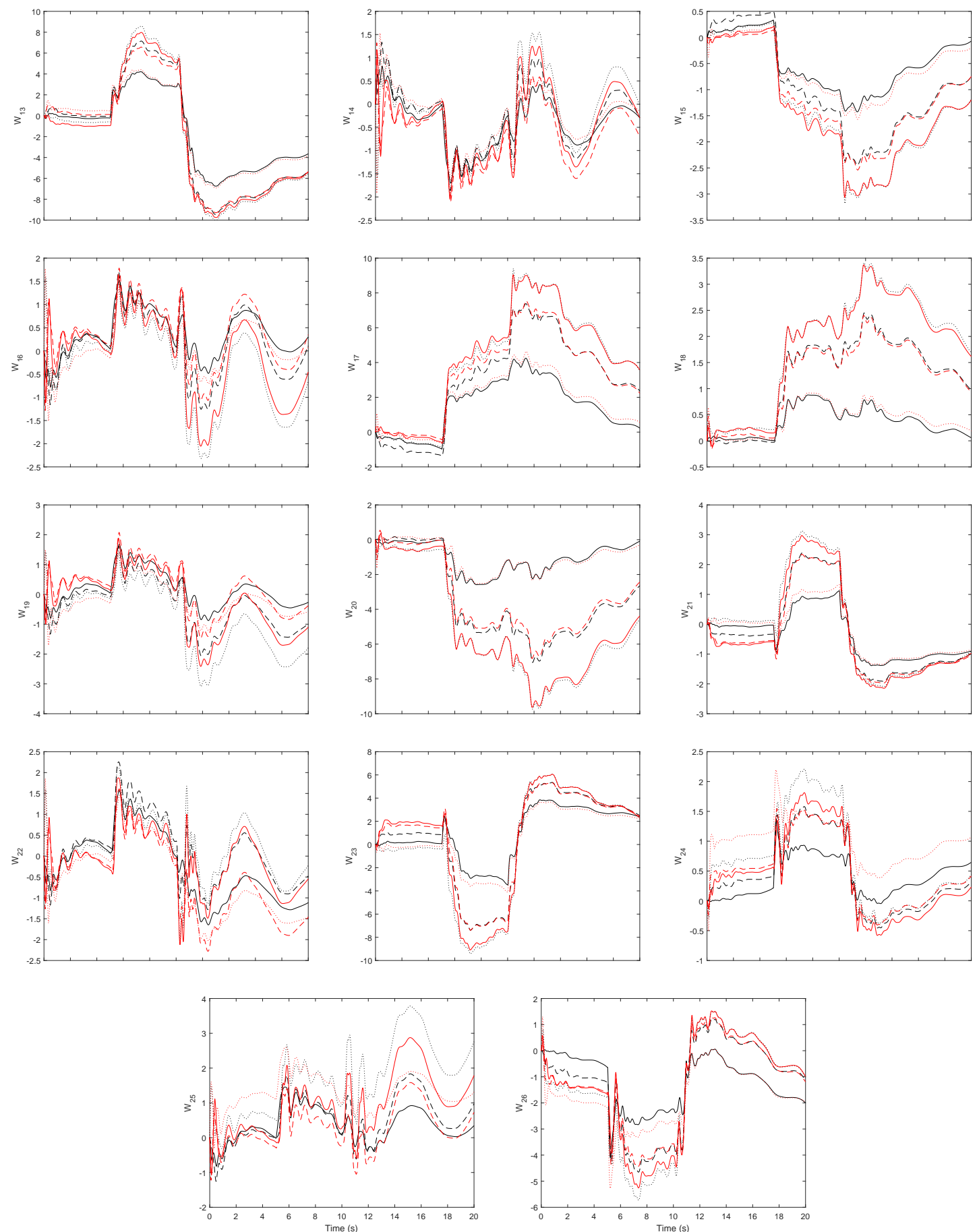

Figure C.3.2: Parameter estimation under external disturbances using marginalized unscented particle filter. 
Appendix C. Parameter Estimation of Chebyshev Neural Network

\section{C.4 Parameter estimation under constant applied con- trol command}
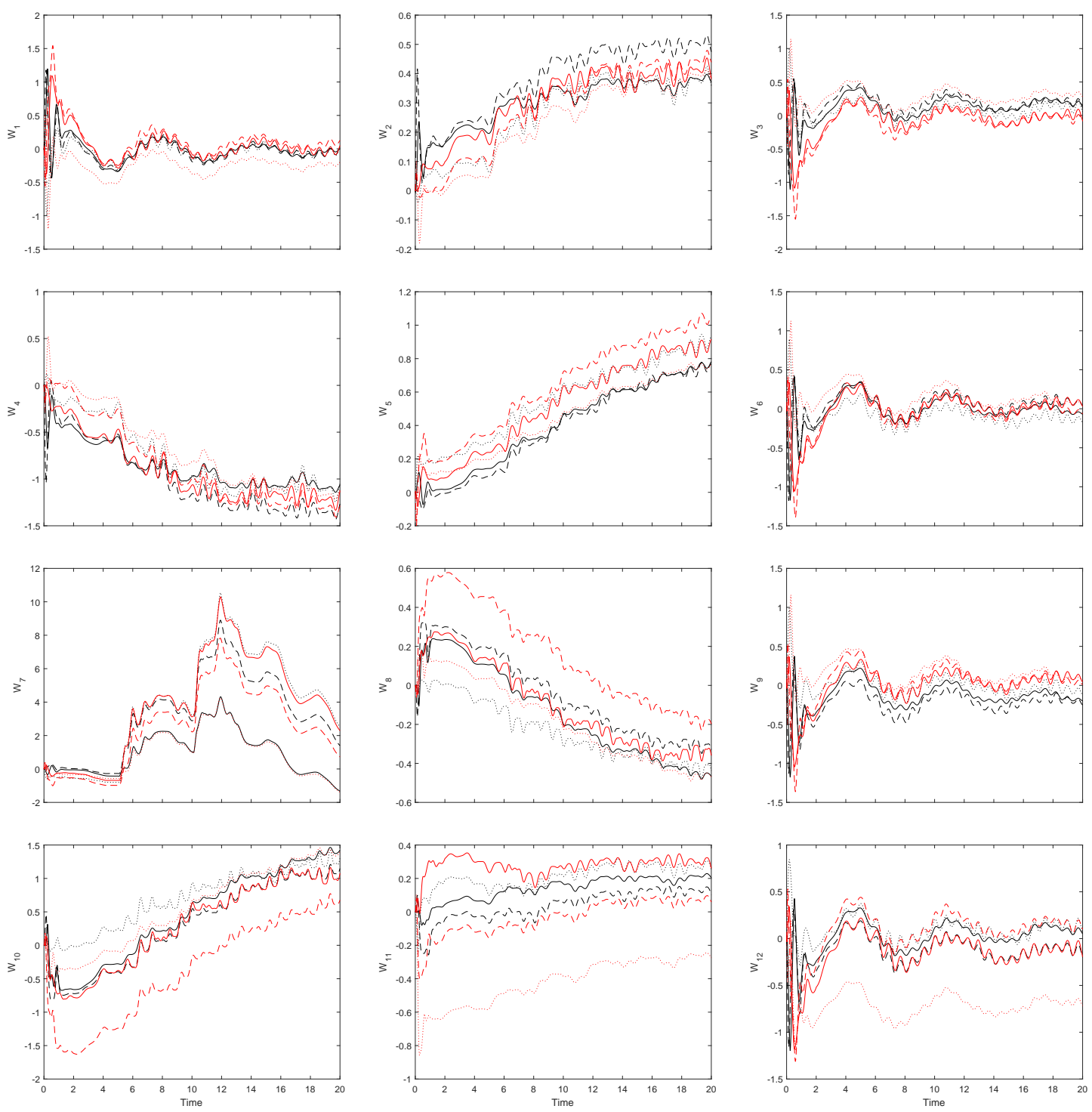


\section{C.4. Parameter estimation under constant applied control command}
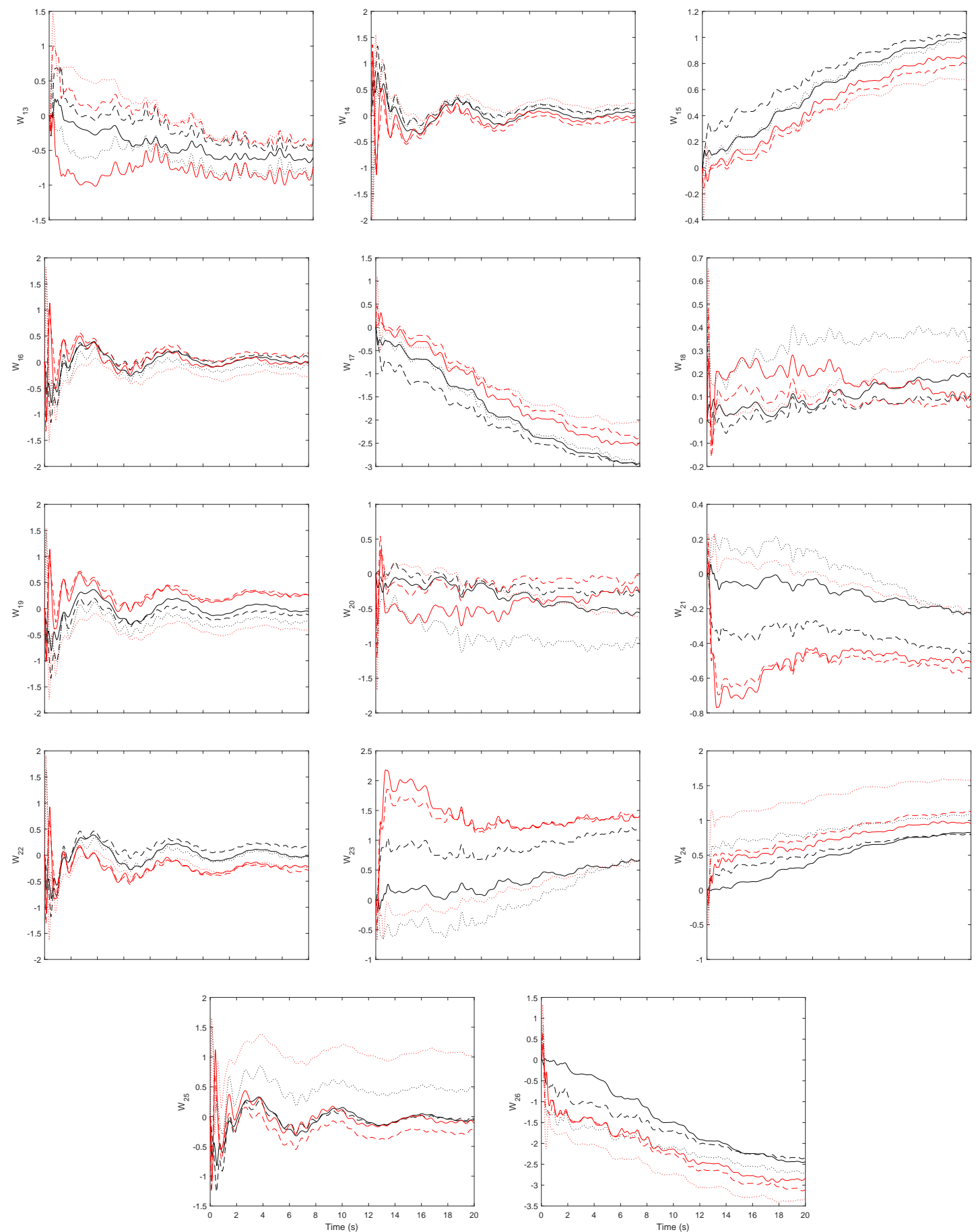

Figure C.4.1: Parameter estimation under constant applied control commands using adaptive unscented Kalman filter. 


\section{Appendix C. Parameter Estimation of Chebyshev Neural Network}
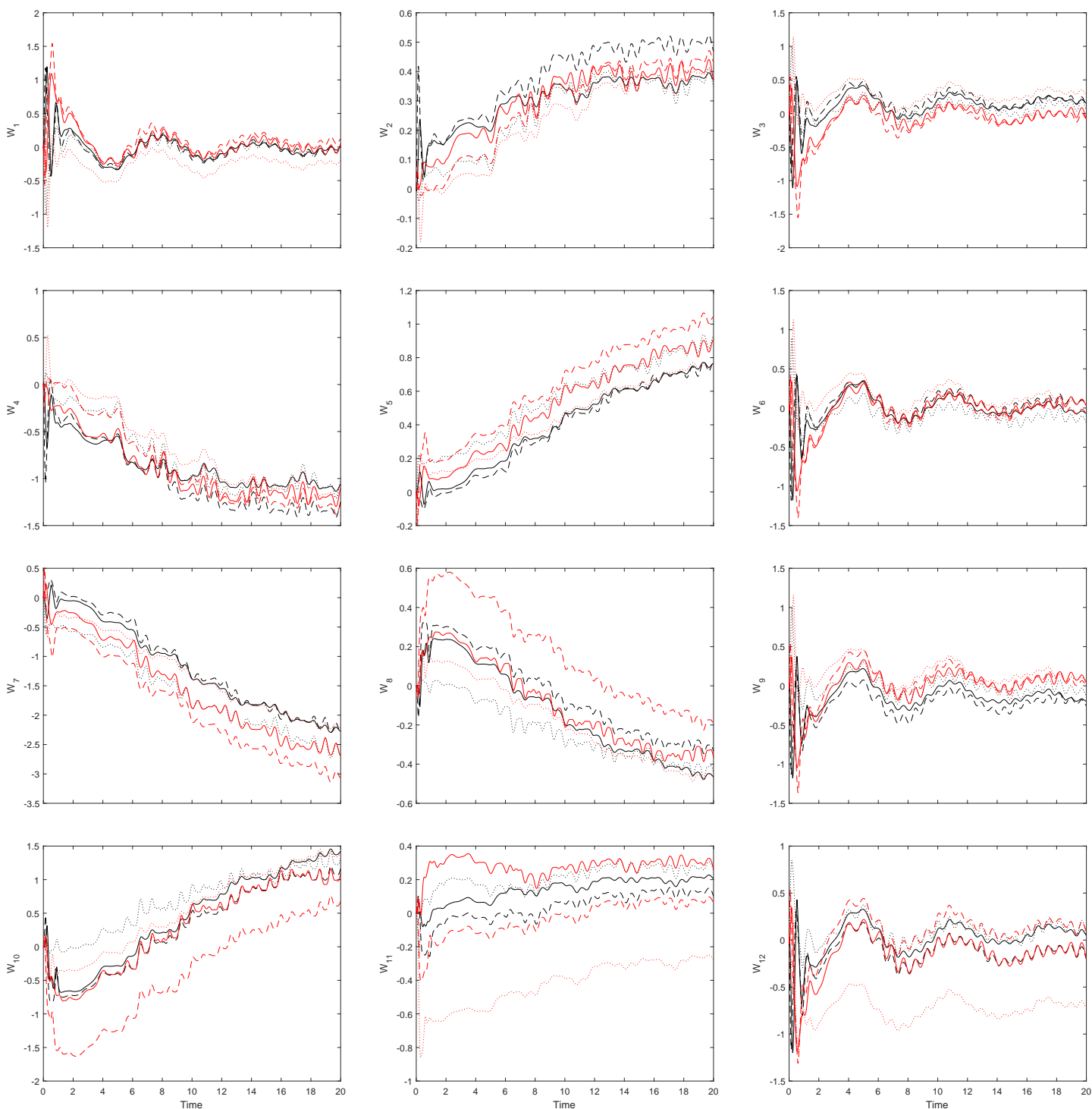


\section{C.4. Parameter estimation under constant applied control command}
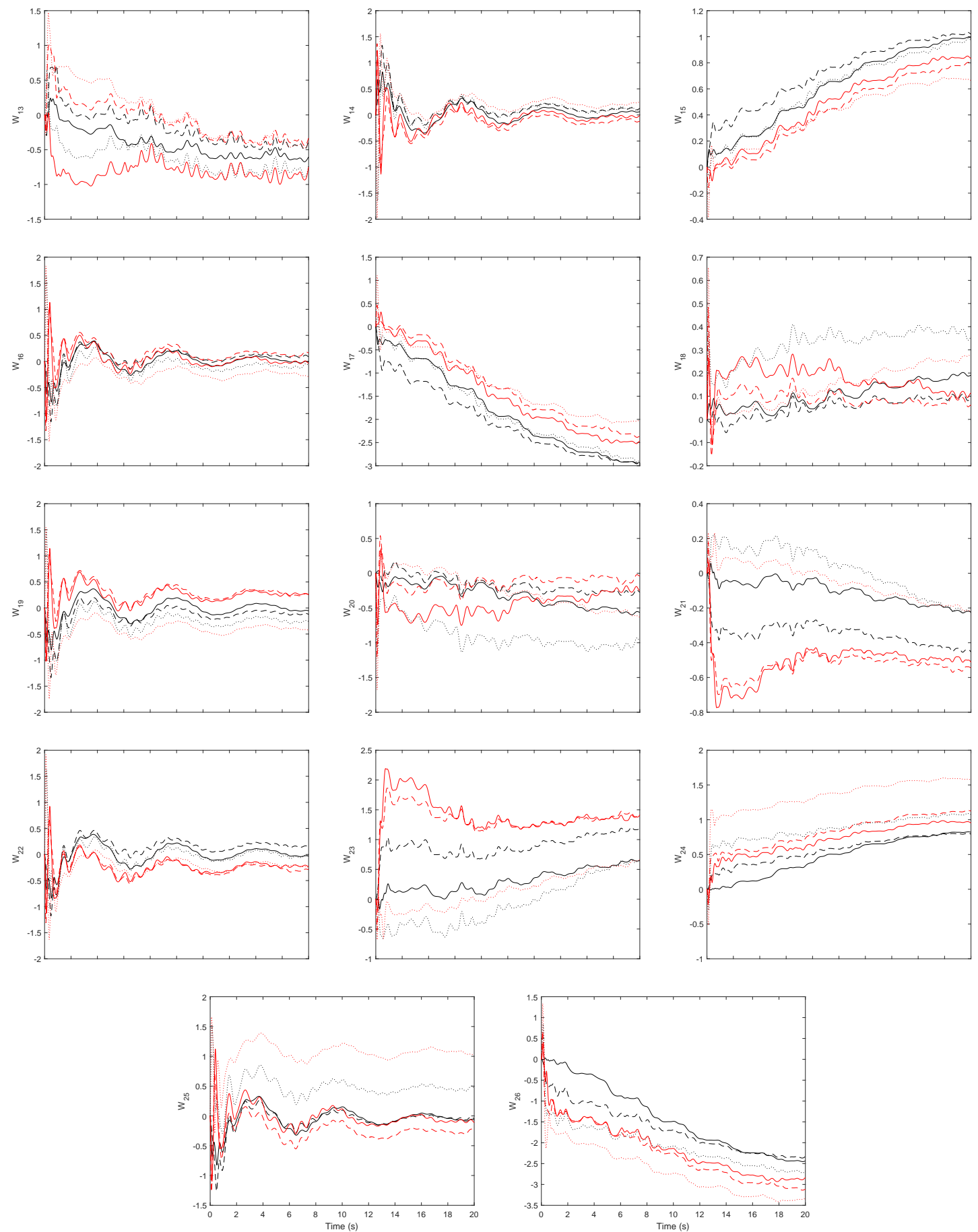

Figure C.4.2: Parameter estimation under constant applied control commands using marginalized unscented particle filter. 


\section{Bibliography}

[Abreu 2011] Abreu, Noel. Fault diagnosis with adaptive kalman filters and CMG design for picosatellite ACS. Master's thesis, Ryerson University, 2011. 24

[Akritas et al. 2000] Akritas, P., Antoniou, I, \& Ivanov, V.V., "Identification and prediction of discrete chaotic maps applying a Chebyshev neural network," Chaos, Solitons \& Fractals, Vol. 11, No. 1, 2000, Pages: 337-344. 33

[Andrieu et al. 2001] Andrieu, Christophe, de Freitas, Nando, \& Doucet, Arnaud, "RaoBlackwellised Particle Filtering via Data Augmentation," Advances in Neural Information Processing Systems 14 [Neural Information Processing Systems: Natural and Synthetic, NIPS 2001, December 3-8, 2001, Vancouver, British Columbia, Canada], 2001, Pages: 561-567. 102

[Arnaud Doucet 2001] Arnaud Doucet, Nando de Freitas, Neil Gordon, Sequential Monte Carlo Methods in Practice, Springer Science+Business Media Inc., 2001. 11, 22, 31

[Arulampalam et al. 2002] Arulampalam, Sanjeev, Simon, Maskell, Neil, Gordon, \& Clapp, Tim, "A Tutorial on Partticle Filters for on-line nonlinear/non-Gaussian Bayesian Tracking," IEEE Transactions on Signal Processing, Vol. 2, 2002, Pages: 174-188. $9,11,46,101$

[Basios et al. 1997] Basios, V., Bonushkina, A.Yu, \& Ivanov, V.V., "A method for approximating one-dimensional functions," Computers $\&$ Mathematics with Applications, Vol. 34, No. 7-8, Oct, 1997, Pages: 687-693. 33

[Battin 1961] Battin, Richard H., "A Statistical Optimizing Navigation Procedure for Space Flight," MIT Instrumentation Laboratory, Vol. R-341, September, 1961. 5

[Battin 1964] Battin, Richard, Astronautical Guidance, McGraw-Hill New York, 1964. 8

[Bishop 1995] Bishop, Christopher M., Neural Networks for Pattern Recognition, Oxford University Press, Inc., New York, NY, USA, 1995. 103 


\section{Bibliography}

[Bucy \& James 1962] Bucy, Richard, \& James, Follin Jr. W., "Adaptive finite time filtering," IRE Transactions on Automatic Control, Vol. 7, No. 4, July, 1962, Pages: $10-19.8$

[Candy 2009] Candy, James V., Bayesian Signal Processing: Classical, Modern, and Particle Filtering Methods, John Wiley \& Sons, Hoboken, New Jersey, USA, 2009. 22

[Carlton \& James 1956] Carlton, AG, \& James, Follin Jr. W., "Recent developments in fixed and adaptive filtering," NATO Advanced Group for Aerospace REDD, AGARDograph, Vol. 21, 1956. 8

[Carpenter et al. ] Carpenter, James, Clifford, Peter, \& Fearnhead, Paul, "An Improved Particle Filter for Non-linear Problems," Department of Statistics, University of Oxford, UK. 86

[Carpenter 2002] Carpenter, J. Russell, "Decentralized control of satellite formations," International Journal of Robust and Nonlinear Control, Vol. 12, No. 2-3, FebruaryMarch, 2002, Pages: 141-161. 78

[Casella \& Robert 1996] Casella, George, \& Robert, Christian P., "Rao-Blackwellisation of sampling schemes," Biometrika, Vol. 83, No. 1, 1996, Pages: 81-94. 102, 104

[Chen \& Liu 2000] Chen, Rong, \& Liu, Jun S., "Mixture Kalman Filters," Journal of the Royal Statistical Society. Series B., Vol. 62, No. 3, 2000, Pages: 493-508. 104

[Chen et al. 2010] Chen, Junli, Wang, Xiaoliang, Shao, Xiaowei, \& Duan, Dengping, "An integrated Relative Navigation system using GPS/VISNAV for ultra-close spacecraft formation flying," 2010 3rd International Symposium on Systems and Control in Aeronautics and Astronautics, 2010, Pages: 803-808. 78

[Chen 2003] Chen, Zhe, "Bayesian Filtering: From Kalman Filters to Particle Filters and Beyond," 2003. 7, 11, 21, 46, 86, 101

[Cheng \& Crassidis 2004] Cheng, Yang, \& Crassidis, John L., "Particle Filtering for Sequential Spacecraft Attitude Estimation," AIAA Guidance, Navigation, and Control Conference and Exhibit, Vol. AIAA 2004-5337, 2004, Pages: 1-18. 40, 41 
[Crisan \& Rozovskii 2011] Crisan, Dan, \& Rozovskii, Boris, The Oxford Handbook of Nonlinear Filtering, Oxford University Press, 2011. 6

[de Freitas 2002] de Freitas, Nando, "Rao-Blackwellised particle filtering for fault diagnosis," IEEE, Aerospace Conference Proceedings, Vol. 4, 2002, Pages: 1767-1772. 104, 109

[Del Moral 1996] Del Moral, Pierre, "Non Linear Filtering: Interacting Particle Solution," Markov Processes and Related Fileds, Vol. 2, No. 4, 1996, Pages: 555-580. 11

[Douc et al. 2005] Douc, Randal, Cappé, Olivier, \& Moulines, Eric, "Comparison of Resampling Schemes for Particle Filtering," . ISSN: 1845-5921, 2005, Pages: 64-69. 46

[Doucet \& Johansen 2008] Doucet, Arnaud, \& Johansen, Adam M., "A tutorial on particle filtering and smoothing: fifteen years later," 2008. 11, 101

[Doucet et al. 2000] Doucet, Arnaud, Freitas, Nando de, Murphy, Kevin P., \& Russell, Stuart J., "Rao-Blackwellised Particle Filtering for Dynamic Bayesian Networks," Proceedings of the 16th Conference on Uncertainty in Artificial Intelligence, UAI '00, San Francisco, CA, USA, 2000, Pages: 176-183. Morgan Kaufmann Publishers Inc. $102,104,153$

[Doucet 1998] Doucet, Arnaud. "On sequential simulation-based methods for bayesian filtering," . Rapport technique, Signal Processing Group, Department of Engineering University of Cambridge CB2 1PZ Cambridge, 1998. 104, 109

[Farrell 1970] Farrell, J.L., "Attitude Determination by Kalman Filtering," Automatica, Vol. 6, 1970, Pages: 419-430. 38

[Fong et al. 2002] Fong, Willian, Godsill, Simon, Doucet, Arnaud, \& West, Mike, "Monte Carlo Smoothing with Application to Audio Signal Enhancement," IEEE Transactions on Signal Procesing, Vol. 50, No. 2, 2002, Pages: 438-449. 104 


\section{Bibliography}

[Gordon \& Smith 1993] Gordon, N.J., Salmond D.J., \& Smith, A.F.M., "Novel approach to nonlinear non-Gaussian Bayesian state estimation," IEEE Proceedings, Vol. 140, No. 2, April 1993, Pages: 3460-3467. 11, 40, 101

[Grewal S. Mohinder 2010] Grewal S. Mohinder, Andrews P. Angus, "Applications of Kalman Filtering in Aerospace 1960 to the Present," IEEE Control Systems Magazine, Vol. 30, No. 3, June, 2010, Pages: 69-78. 9, 38

[Halton 1962] Halton, John H, "Sequential monte carlo," Mathematical Proceedings of the Cambridge Philosophical Society, Vol. 58. Cambridge Univ Press, 1962, Pages: 5778. 152

[Haug 2005] Haug, A.J. "A tutorial on Bayesian Estimation and Tracking Techniques Applicable to Nonlinear and Non-Gaussian Processes," . Rapport technique Case 05-0211, MITRE Corporation Technical Report, February, 2005. 86

[Hol et al. 2007] Hol, Jeroen, Schön, Thomas, \& Gustafsson, Fredrik. "On Resampling Algorithms for Particle Filters," . Technical report LiTH-ISY-R-2766, Automatic Control at Linköpings Universitety, January, 2007. 46

[Hughes 1986] Hughes, P. C., Spacecraft attitude dynamics, Wiley, New York, 1986. 42, 82

[Jazwinski 1970] Jazwinski, Andrew H., Stochastic Processes and Filtering Theory, 1970. 19

[Jin \& Sun 2008] Jin, Erdong, \& Sun, Zhaowei, "Robust controllers design with finite time convergence for rigid spacecraft attitude tracking control," Aerospace Science and Technology, Vol. 12, No. 4, 2008, Pages: 324-330. 43

[Johansen et al. 2012] Johansen, Adam M., Whiteley, Nick, \& Doucet, Arnaud, "Exact approximation of Rao-Blackwellised particle filters," 16th IFAC Symposium on System Identification, SYSID 2012, Brussels, Belgium. Published in: System Identification, Vol.16 (No.1). pp. 488-493, July, 2012. 104 
[Julier \& Uhlmann 1997] Julier, Simon J., \& Uhlmann, Jeffrey K., "New extension of the Kalman filter to nonlinear systems," Proceedings of the SPIE - The International Society for Optical Engineering, Vol. 3068, 1997, Pages: 182-193. 10, 22, 101

[Kalman \& Bucy 1961] Kalman, R.E, \& Bucy, R.S., "New Result in Linear Filtering and Prediction Theory," Transactions of ASME, Series D, Journal of Basic Engineering, Vol. 83, March, 1961, Pages: 95-107. 8

[Kalman 1960] Kalman, R.E, "A New Approach to Linear Filtering and Prediction Problem," Transactions of ASME Series D, Journal of Basic Engineering, Vol. 82, March, 1960, Pages: 34-45. 5, 8

[Kang et al. 2001] Kang, Bryan H., Hadaegh, Fred Y., \& Scharf, Daniel P., Ke NainPing, "Decentralized and self-centered estimation architecture for formation flying of spacecraft," 16th International Symposium on Space Flight Dynamics; Pasadena, CA, United States, December, 2001. 76

[Kim et al. 2007] Kim, Son-Goo, Crassidis, John L., Cheng, Yang, Fosbury, Adam M., \& Junkins, John L., "Kalman Filtering for Relative Spacecraft Attitude and Position Estimation," Journal of Guidance, Control, and Dynamics, Vol. 30, No. 1, 2007, Pages: 133-143. 78

[Kitagawa 1996] Kitagawa, Genshiro, "Monte Carlo filter and smoother for non-Guassian non-linear state space models," Journal of Computational and Graphical Statistics, Vol. 5, 1996, Pages: 1-25. 11, 86

[Kitagawa 1998] Kitagawa, Genshiro, "A self-orgenizing state-space model," Journal of Americal Statistical Associateion, Vol. 93, 1998, Pages: 1203-1215. 11

[Lee \& Jeng 1998] Lee, Tsu-Tian, \& Jeng, Jin-Tsong, "The Chebyshev-Polynomials-Based Unified Model Neural Networks for Function Approximation," IEEE Transactions on Systems, Man, and Cybernatics, Part B: Cybernatics, Vol. 28, No. 6, December, 1998, Pages: 925-935. 33 


\section{Bibliography}

[Lefferts et al. 1982] Lefferts, E., Markley, F., \& Shuster, M., "Kalman Filtering for Spacecraft Attitude Estimation," Journal of Guidance, Control, and Dynamics, Vol. 5, No. 5, September-October, 1982, Pages: 417-429. 38

[Lin 2014] Lin, Arthur Kar Leaung. "Control of Spacecraft Formation Flying Around Asteriods," . PhD thesis, Ryerson University, 2014. 103

[Lin 2015] Lin, Chih-Hong, "Adaptive recurrent Chebyshev neural network control for PM synchronous motor servo-drive electric scooter with V-belt continuously variable transmission," International Journal of Adaptive Control and Signal Processing, Vol. 29, 2015, Pages: 805-834. 33

[Liu \& Chen 1998] Liu, Jun S., \& Chen, Rong, "Sequential Monte Carlo Methods for Dynamic Systems," Journal of the American Statistical Association, Vol. 93, 1998, Pages: 1032-1044. 32

[Liu 2008] Liu, Jun S., Monte Carlo Strategies in Scientific Computing, Springer Publishing Company, Incorporated, 2008. 31

[Mall \& Chakraverty 2014] Mall, Susmita, \& Chakraverty, S., "Chebyshev Neural Network Based Model for Solving Lane-Emden Type Equations," Appl. Math. Comput., Vol. 247, No. C, nov, 2014, Pages: 100-114. 33

[Mark L. Psiaki 1990] Mark L. Psiaki, Francois Martel, Parimal K. Pal, "Three-axis attitude determination via Kalman filtering of magnetometer data," Journal of Guidance, Control, and Dynamics, Vol. 13, No. 3, 1990, Pages: 506-514. 38

[Martin E. Liggines 2009] Martin E. Liggines, David L. Hall, James Llinas, Handbook of Multisensor Data Fusion: Theory and Practice, 2nd ed., CRC Press, Taylor \& Francis Group, Boca Raton, FL, USA, 2009. 8, 20

[Metropolis \& Ulam 1949] Metropolis, Nicholas, \& Ulam, Stanislaw, "The Monte Carlo Method," Journal of the American Statistical Association, Vol. 44, No. 247, September, 1949, Pages: 335-341. 25 
[Metropolis 1987] Metropolis, Nicholas, "The Begining of the Monte Carlo Method," Los Almos Science, Special Issue, Stanislaw Ulam 1909-1984, No. 15, 1987, Pages: 125130. 25

[Murphy \& Russell 2001] Murphy, Kevin, \& Russell, Stuart, Rao-Blackwellised Particle Filtering for Dynamic Bayesian Networks, A. Doucet, J. F. G. de Freitas, and N. J. Gordon, Springer-Verlag, New York, NY, 2001. 102

[Nebelecky et al. 2009] Nebelecky, Christopher, Crassidis, John, Banas, William, Cheng, Yang, \& Fosbury, Adam, "Decentralized Relative Attitude Estimation for ThreeSpacecraft Formation Flying Applications," AIAA Guidance, Navigation, and Control Conference, Chicago, Illinois, August 2009. 78

[Nikolas Trawny 2005] Nikolas Trawny, Stergios I. Roumeliotis. "Indirect Kalman Filter for 3D Attitude Estimation: A Tutorial for Quaternion Algebra," . Rapport technique TR-2005-002, Rev. 57, Department of Computer Science \& Engineering, University of Minnesota, Multiple Autonomous Robotic Systems Laboratory, March, 2005. 38

[Nordlund \& Gustafsson 2009] Nordlund, Per-Johan, \& Gustafsson, Fredrik, "Marginalized Particle Filter for Accurate and Reliable Terrain-Aided Navigation," IEEE Transactions on Aerospace and Electronic Systems, Vol. 45, No. 4, 2009, Pages: 1385-1399. 104

[Patra \& Kot 2002] Patra, Jagdish Chandra, \& Kot, Alex ChiChung, "Nonlinear dynamic system identification using Chebyshev functional link artificial neural networks," IEEE Trans. Systems, Man, and Cybernetics, Part B, Vol. 32, 2002, Pages: 505511. 33

[Paul Zarchan 2000] Paul Zarchan, Howard Musoff, Fundamentals of Kalman Filtering: A Practical Approach, Progress in Astronautics and Aeronautics, AIAA, third édition, 2000. 8

[Poggio \& Girosi 1990] Poggio, Tomaso, \& Girosi, Federico, "Networks for approximation and learning," Proceedings of the IEEE, Vol. 78, No. 9, 1990. 34 


\section{Bibliography}

[Purwar et al. 2007] Purwar, S., Kar, I. N., \& Jha, A. N., "On-line System Identification of Complex Systems Using Chebyshev Neural Networks," Applied Soft Computing, Vol. 7, No. 1, January, 2007, Pages: 364-372. 33

[Rapoport \& Oshman 2004] Rapoport, Ilia, \& Oshman, Yaakov, "Fault Tolerant Particle Filtering Using IMM-Based Rao-Blackwellization," AIAA Guidance, Navigation, and Control Conference and Exhibit, Vol. AIAA 2004-5416, 2004, Pages: 1-13. 39

[Ren 2007] Ren, Wei, "Distributed attitude alignment in spacecraft formation flying," International Journal of Adaptive Control and Signal Processing, Vol. 21, 2007, Pages: 95-113. 87

[Robert \& Casella 2005] Robert, Christian P., \& Casella, George, Monte Carlo Statistical Methods (Springer Texts in Statistics), Springer-Verlag New York, Inc., Secaucus, NJ, USA, 2005. 31

[Rogers 2007] Rogers, Robert M., Applied Mathematics in Integrated Navigation Systems, AIAA Inc., third edition, 2007. 22

[Rumelhart et al. 1986] Rumelhart, D. E., Hinton, G. E., \& Williams, R. J. "Parallel Distributed Processing: Explorations in the Microstructure of Cognition, Vol. 1," . Chapter: Learning Internal Representations by Error Propagation, Pages: 318-362. MIT Press, Cambridge, MA, USA, 1986. 103

[Sahnow et al. 2006] Sahnow, David J., Kruk, Jeffrey W., Ake, Thomas B., Andersson, B-G, Berman, Alice, Blair, William P., Boyer, Robert, Caplinger, James, Calvani, Humberto, Civeit, Thomas, Dixon, W. Van Dyke, England, Martin N., Kaiser, Mary Elizabeth, Kochte, Mark, Moos, H. Warren, \& Roberts, Bryce A., "Operations with the new FUSE observatory: three-axis control with one reaction wheel," . Vol. 6266, 2006. 2

[Särkkä et al. 2007] Särkkä, Simo, Vehtari, Aki, \& Lampinen, Jouko, "Rao-Blackwellized Particle Filter for Multiple Target Tracking," Information Fusion, Vol. 8, No. 1, January, 2007, Pages: 2-15. 104 
[Särkkä 2013] Särkkä, Simo, Bayesian Filtering and Smoothing, IMS Textbook Series, Vol. 3, Cambridge University Press, 2013. 22, 30, 102, 104, 109

[Schmidt 1981] Schmidt, Stanley F., "The Kalman Filter: Its Recognition and Development for Aerospace Applications," Journal of Guidance and Control, Vol. 4, No. 1, 1981, Pages: 4-7. 6,9

[Schön et al. 2006] Schön, Thomas B., Gustafsson, Fredrik, \& Karlsson, Rickard, "The marginalized particle filter in practice," Proceedings of IEEE Aerospace Conference, Big Sky, 2006. 104

[Shuster 1993] Shuster, M. D., "A survey of attitude representations," Journal of Astronautical Sciences, Vol. 41, No. 4, 1993, Pages: 439-517. 43, 82

[Simon 2006] Simon, Dan, Optimal State Estimation: Kalman, $H_{\infty}$, and Nonlinear Approaches, John Wiley \& Sons Inc., Hoboken, New Jersey, 2006. 8

[van der Merwe et al. 2000] van der Merwe, Rudolph, Doucet, Arnaud, de Freitas, Nando, \& Wan, Eric. "The Unscented Particle Filter," . Rapport technique CUED/FINFENG/TR 380, Cambridge University Engineering Department, August, 2000. 147

[Vathsal 1987] Vathsal, S., "Spacecraft Attitude Determination Using a Second-Order Nonlinear Filter," Journal of Guidance, Control, and Dynamics, Vol. 10, No. 6, 1987, Pages: 559-566. 39

[Vihola 2007] Vihola, Matti, "Rao-blackwellised particle filtering in random set multitarget tracking," IEEE Transactions on Aerospace and Electronic Systems, Vol. 43, April, 2007, Pages: 689-705. 104

[W. H. Moos 2000] W. H. Moos, W. C. Cash, L. L. Cowie A. F. Davidsen A. K. Dupree P. D. Feldman S. D. Friedman, "Overview Of The Far Ultraviolet Spectroscopic Explorer Mission," The Astrophysical Journal, Vol. 538 L1-L6, July, 2000. 2 


\section{Bibliography}

[Wan \& Van Der Merwe 2000] Wan, Eric A, \& Van Der Merwe, Rudolph, "The unscented Kalman filter for nonlinear estimation," Adaptive Systems for Signal Processing, Communications, and Control Symposium 2000. AS-SPCC. The IEEE 2000. IEEE, 2000, Pages: 153-158. 10, 24, 147

[Wan-Chun Li 2005] Wan-Chun Li, Ping Wei, Xian-Ci Xiao, "An adaptive nonlinear filter of discrete-time system with uncertain covariance using unscented Kalman filter," International Symposium on Communications and Information Technologies 2005, Proceedings, Vol. 1, 2005, Pages: 1389-1392. 22

[Wang \& Chaib-draa 2012] Wang, Yali, \& Chaib-draa, Brahim, "An Adaptive Nonparametric particle Filter for State Estimation," 2012 IEEE International Conference on Robotics and Automation (RiverCentre, Saint Paul, Minniesota, USA), May 14-182012, Pages: 4355-4360. 31

[Wiener Norbert 1949] Wiener Norbert, 1894-1964, Extrapolation, Interpolation, and Smoothing of Stationary Time Series, with Engineering Applications, [Cambridge] Technology Press of the Massachusetts Institute of Technology. Note: First published during the war as a classified report to Section D2, National Defense Research Committee., 1949. 5, 18

[Wiener Norbert 1956] Wiener Norbert, 1894-1964, I am a Mathmatician: The later Life of Prodigy, Doubleday \& Company, Inc., Garden City, New-York, 1956. 17

[Wong 2001] Wong, H., de Queiroz M. S.-Kapila V., "Adaptive tracking control using synthesized velocity from attitude measurements," Automatica, Vol. 37, No. 6, 2001, Pages: 947-953. 45

[Xing et al. 2010] Xing, Yanjun, Cao, Xibin, Zhang, Shijie, Guo, Haibo, \& Wang, Feng, "Relative position and attitude estimation for satellite formation with coupled translational and rotational dynamics," Acta Astronautica, No. 67, April, 2010, Pages: 455-467. 78 
[Xiong K. 2007] Xiong K., Chan C.W., Zhang H.Y., "Detection of satellite attitude sensor faults using the UKF," IEEE Transactions on Aerospace and Electronic Systems, Vol. 43, No. 2, April, 2007, Pages: 480-491. 39

[Zakai 1965] Zakai, Moshe, "The Optimal Filtering of Markov Jump Processes in Additive White Noise," Applied Research Laboratory Sylvania Electronic System, Vol. Research Note No.563, 1965. 5

[Zakai 1969] Zakai, Moshe, "On the optimal filtering of diffusion processes," Zeitschrift für Wahrscheinlichkeitstheorie und Verwandte Gebiete, Vol. 11, No. 3, 1969, Pages: 230-243. 5

[Zhang et al. 2015] Zhang, Lijun, Li, Tong, Yang, Huabo, Zhang, Shifeng, Cai, Hong, \& Qian, Shan, "Unscented Kalman Filtering for Relative Spacecraft Attitude and Position Estimation," Journal of Navigation, Vol. 68, No. 03, 2015, Pages: 528-548. 79

[Zou et al. 2011] Zou, An-Min, Kumar, Krishna-Dev, \& Hou, Zeng-Guang, "Distributed Consensus Control for Multi-Agent Systems Using Terminal Sliding Mode and Chebyshev Neural Networks," International Journal of Robust and Nonlinear Control, Vol. 23, 2011, Pages: 334-357. 103, 111, 114

[Zou et al. 2014] Zou, An-Min, Kumar, Krishna-Dev, \& de Ruiter, Anton H.J., "Quaternion-based output feedback attitude control for rigid spacecraft with finitetime convergence," December, 2014. 44

[Zou et al. 2016] Zou, An-Min, de Ruiter, Anton H. J., \& Kumar, Krishna-Dev, "Distributed finite-time velocity-free attitude coordination control for spacecraft formations," Automatica, Vol. 67, 2016, Pages: 46-53. 84, 85

[Zou 2014] Zou, An-Min, "Finite-Time Output Feedback Attitude Tracking Control for Rigid Spacecraft," IEEE Transaction on Control Systems Technology, Vol. 22, No. 1, January, 2014, Pages: 338-345. 82 